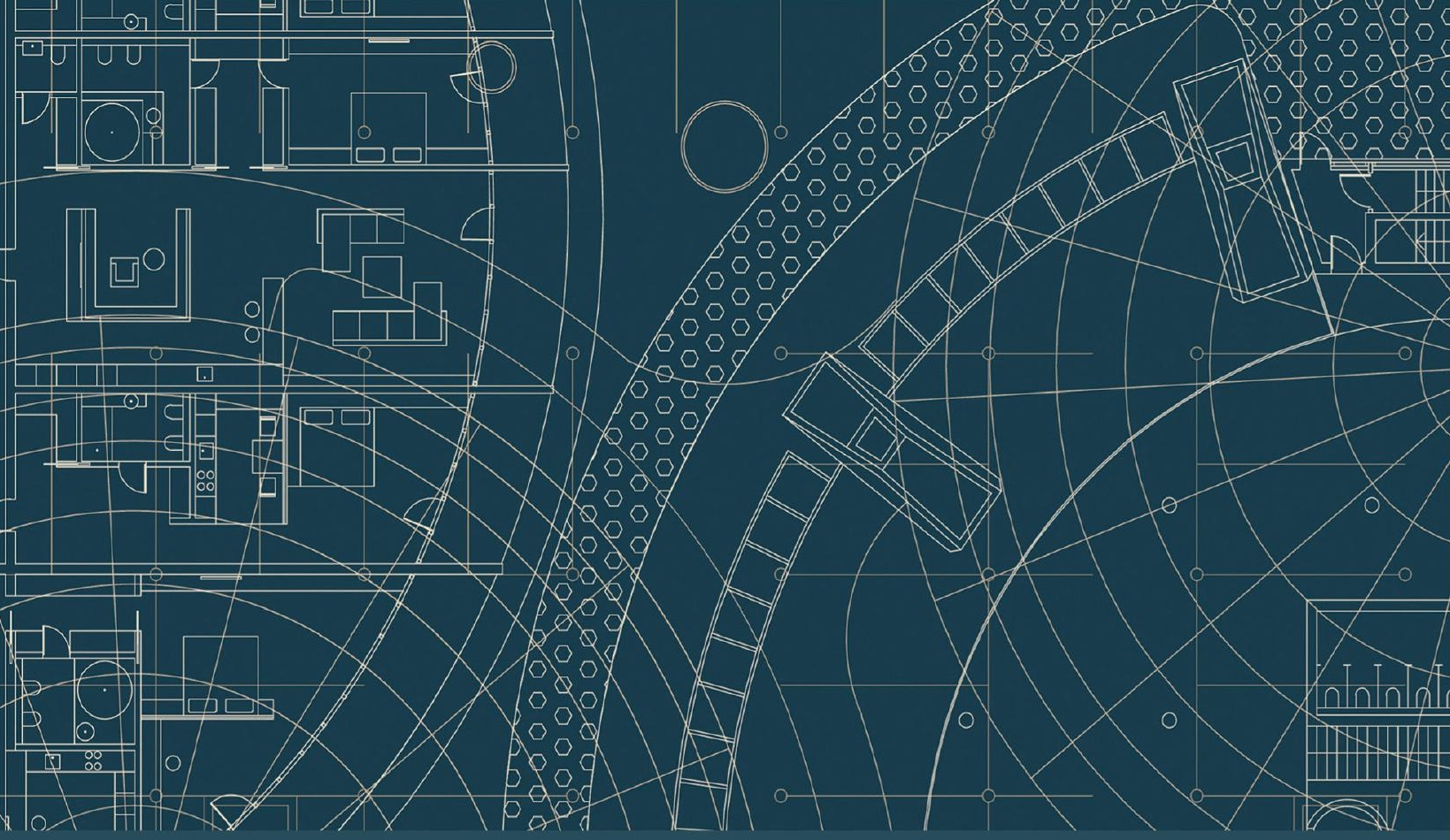

Routledge Studies in Science, Technology and Society

\title{
QUESTING EXCELLENCE IN ACADEMIA
}

\section{A TALE OF TWO UNIVERSITIES}

Knut H. Sørensen and Sharon Traweek

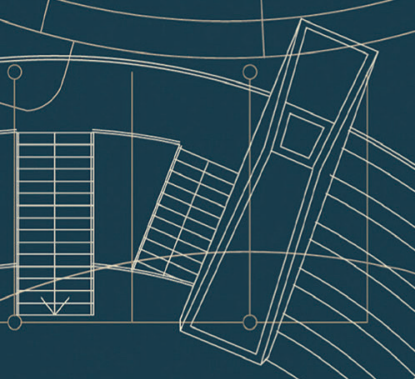




\section{Questing Excellence in Academia}

Unlike almost most other studies of neoliberal universities and academic capitalism this book ethnographically explores and interprets those transformations and their contradictions empirically in the everyday practices of students, faculty members, and administrators at two public universities: NTNU in Norway and UCLA in California.

Differently situated in global political economies, both are ambitious, prosperous campuses. The book reflexively examines their disturbing disputes about quality, competition, and innovation. It argues that some academic, bureaucratic, and corporate university governance practices are both unsustainable and undermining what some university students and faculty already do well: circulate interdisciplinary knowledge and its making globally across the diasporic domains of academia, society, industry, and government while addressing the world's immediate challenges: power, inequities, and sustainability.

It shows the important, strategic work of domesticating, co-morphing, and meshworking at the faultlines of emerging knowledge. This book is for students, faculty, society members, and policy makers who want to engage more effectively with contemporary universities that increasingly serve as busy crossroads for sharing ideas and how to make them. It will be of interest to workers and scholars in the interdisciplinary fields of higher education studies, critical university studies, and critical public infrastructure studies, plus science, technology, and society studies.

Knut H. Sørensen is Professor Emeritus at NTNU, Department of Interdisciplinary Studies of Culture and Centre for Technology and Society. His academic work has spanned many areas of study, including climate and energy studies, feminist technoscience, and interdisciplinarity.

Sharon Traweek is Associate Professor of Gender Studies at UCLA and holds the Bernal Prize for her distinguished contributions to Science, Technology, and Society studies. She conducts research and teaches about knowledge making practices among anthropologists, astronomers, historians, and physicists in Japan, Sweden, Switzerland, and the US. 
Routledge Studies in Science, Technology and Society

40 The Cultural Authority of Science

Comparing across Europe, Asia, Africa and the Americas

Edited by Martin W Bauer, Petra Pansegrau, Rajesh Shukla

4I Blockchain and Web 3.0

Social, Economic, and Technological Challenges

Edited by Massimo Ragnedda and Giuseppe Destefanis

42 Understanding Digital Events

Bergson, Whitehead, and the Experience of the Digital

Edited by David Kreps

43 Big Data-A New Medium?

Edited by Natasha Lushetich

44 The Policies and Politics of Interdisciplinary Research

Nanomedicine in France and in the United States

Séverine Louvel

45 Apocalyptic Narratives

Science, Risk and Prophecy

Hauke Riesch

46 Distributed Perception

Resonances and Axiologies

Edited by Natasha Lushetich and lain Campbell

47 Questing Excellence in Academia

A Tale of Two Universities

Knut H. Sørensen and Sharon Traweek

For the full list of books in the series: www.routledge.com/Routledge-Studies-inScience-Technology-and-Society/book-series/SE0054 


\title{
Questing Excellence in Academia
}

\section{A Tale of Two Universities}

\author{
Knut H. Sørensen
} and Sharon Traweek 
First published 2022

by Routledge

2 Park Square, Milton Park, Abingdon, Oxon OXI4 4RN

and by Routledge

605 Third Avenue, New York, NY 10158

Routledge is an imprint of the Taylor \& Francis Group, an informa business

(C) 2022 Knut H. Sørensen and Sharon Traweek

The right of Knut H. Sørensen and Sharon Traweek to be identified as authors of this work has been asserted in accordance with sections 77 and 78 of the Copyright, Designs and Patents Act 1988.

The Open Access version of this book, available at www.taylorfrancis.com, has been made available under a Creative Commons Attribution-Non Commercial-No Derivatives 4.0 license.

Trademark notice: Product or corporate names may be trademarks or registered trademarks, and are used only for identification and explanation without intent to infringe.

British Library Cataloguing-in-Publication Data

A catalogue record for this book is available from the British Library

Library of Congress Cataloging-in-Publication Data

A catalog record has been requested for this book

ISBN: 978-0-367-25933-4 (hbk)

ISBN: 978-I-032-I5267-7 (pbk)

ISBN: 978-0-429-29063-3 (ebk)

DOI: 10.4324/9780429290633

Typeset in Times New Roman

by Newgen Publishing UK 


\section{Contents}

Preface

$i x$

1 Navigating Universities, University Studies, and This Book

Introduction 1

Disciplining Universities to Provide Public Goods 5

Universities, the Public Good, and Their Quests for Excellence 9

Navigating the Field of University Studies 13

Reconfiguring University Studies 16

Homeward Bound: Engaging Ethnographically with Universities 18

Questing, Resisting, and Re-morphing Excellence 22

Intervening 23

Locating Two Universities 26

NTNU 26

UCLA 28

A Brief Outline of This Book 33

2 Disciplining Universities: Performance Metrics, Policy Reforms, and Reputations

Introduction 36

Distinctions: The Role of National Governments in Governing

Universities and the New Metric Managerial Models for

Universities 38

Corporatizing and Bureaucratizing Are Not in Opposition 40

Building University Reputations: Branding and Ranking 42

Branding as a Strategy for Building a Distinctive Reputation 42

University Ranking Systems 44

Presences and Absences: Features of the Political Economies of NTNU and UCLA 46

NTNU - a Bureaucratizing University with Government Presence 47

UCLA - a Corporatizing University 54

Conclusion: The Co-morphing of Metrics and 'Excellence' 65 
3 In the Shadows of Excellence and Neoliberal Interventions: Enactments of Academic Autonomy and Strained Collegiality

Introduction 69

Universities as Hierarchies 72

Extramural Circulation 76

Academic Freedom, Autonomy, and Epistemic Politics 78

Transformations: Growth, Democracy, and Resilience 80

Corporatizing and Bureaucratizing as Circumscriptions of

Academic Life 83

Decision making with Factions 87

Factions, Conflicts, and Receivership 90

Factions and Pawa Hara 91

Enactments of and Limits to Academic Freedom 95

Academic Autonomy and the Regime of Meritocracy 99

Academic Citizenship as Legitimizing Academic Freedom 101

Epistemic Politics and Collegial Relations 104

Conclusion 105

4 Subject Formation and Re-formation Throughout

Academic Careers: The Double Bind of Disciplined

Entrepreneurs

Introduction 108

Entrepreneurship in Academia 111

Subject Formation in Academia 112

Becoming Academics in the US: Disciplined and Entrepreneurial

Epistemic Choices - Sharon's Account 114

Strategic Choices: Place and Pace 117

Applying to Graduate Schools in the US: Strategically

Selecting Reputations 119

Becoming a Graduate Student 121

Selecting Research Topics, Pedagogies, and Mentors:

Disciplined or Entrepreneurial? 123

Funding Ecologies or Access to the Means of Production of Our Work: Teaching + Research 127

Working in 'The College' 130

Hiring Colleagues 131

Strategic Mobility and Negotiating 134

Becoming an Entrepreneurial Academic 135

Becoming Evaluated 139

Becoming Academics in Norway - Knut's Account 146

Employment and Admission 149

Transformations 150 
Getting Out of Limbo 152

Assessment Experiences 153

Systems of Formation of Academic Subjects 155

5 The Meshworking University: The Traffic in Knowledge through Teaching, Innovation, and Interdisciplinarity Practices

Introduction 158

Transmitting and Transforming Research Through Teaching 163

Regulating Quality 167

Academics Circulating Through Government, and Industry 170 Innovation - a Third Mission 172

Interdisciplinarity and the Epistemic Angst of Disciplines 177

Conclusion: Flows and Deficits 181

6 Conclusion: Questing a Sustainable University

Introduction 184

Our Indicators, Ourselves 186

University Governance: Bureaucratizing at NTNU and Corporatizing at UCLA 190

Academic and Administrative Cultures: Stylized Differences and Indifferences Persist 192

University Governance, Gossip, Meshworks, and Change 197

Knowledge Traffic for Sustainability Transitions 200

Engaging with STS and University Studies: Some Final

Reflections on Studying Unsustainable Universities 202

References and Further Reading

Name Index

Keyword Index 


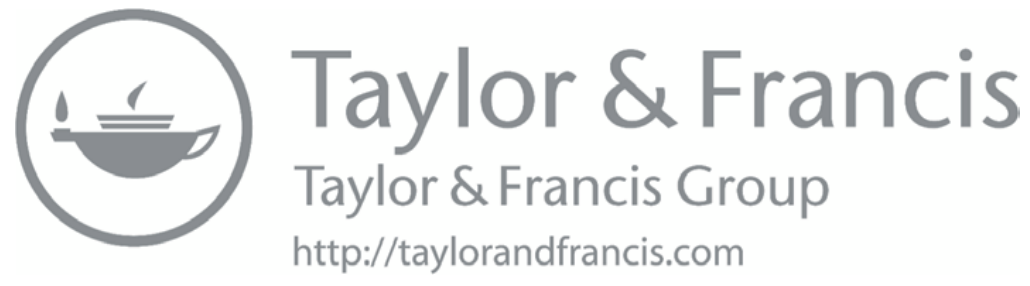




\section{Preface}

It is hard to say when our collaboration on this book began. We met in 1983 at MIT and since then have been in active conversation about universities, interdisciplinarity, justice, sustainability, teaching, Norway, California, and much more. We regularly surprise, confound, agree, and confuse each other. Finishing this book will not end our provocative dialogues.

Our discussions about the particular issues raised in the book began while Knut was a visiting researcher at UCLA's Institute of the Environment and Sustainability in 2017 and continued through a series of conversations at the annual conferences of the international, interdisciplinary Society for Social Studies of Science, as well as some visits at NTNU and UCLA. We have had bi-weekly Skype and Zoom meetings for a long time. Due to the Covid-19 pandemic, we could not meet in person, which has delayed us. During the last several weeks of generating this manuscript we made use of our nine-hour time difference: one would send a draft at the end of a day near the Pacific and then many hours later the other would send a subsequent version from near the North Sea.

This book joins an ongoing, global debate about the crisis in universities and knowledge making. We have benefitted profoundly from that scholarship and are grateful for it. A full, annotated bibliography of that interdisciplinary literature would be a great resource for all of us. We apologize for citing only a tiny amount of the work that has inspired us. We are eager for the conversations we will be pursuing with you about this book and your work.

Knut's work with the book has benefitted from participating in two ongoing projects both funded by the Research Council of Norway: GENDIM Understanding gender imbalances among university professors: the shaping and reshaping of epistemic living spaces (grant no. 296173) and Res Publica: Responsibility, practice, and the public good across Digital Life (grant no. 270623). He is particularly grateful for conversations regarding these projects with Ivana Suboticki, Vivian Anette Lagesen and Heidrun Åm: Vivian, in particular, allowed Knut to use data from the GENDIM 
project. He also wants to thank Margrethe Aune and Nora Levold for yearlong exchanges about universities.

Sharon wants to thank five people who have sustained her intellectually, socially, and personally for decades, including during the writing of this book: Kim Fortun and Nadine Tanio of the University of California at Irvine, Diane Yu Gu of UCLA, Jarita Holbrook of the University of Edinburgh, and Wendy Martin of Claremont Graduate University. Kim, Nadine, and Diane are former students and Jarita once worked with Sharon as a postdoc; each began teaching the teacher from the first day we met. Sharon and Wendy met as undergraduates at the University of California at Berkeley.

We gratefully acknowledge the economic support from NTNU that has enabled the open access publication of this book.

Knut H. Sørensen

Sharon Traweek

Los Angeles/Trondheim July 2021 


\section{Navigating Universities, University Studies, and This Book}

\section{Introduction}

For generations, universities have been institutions providing higher education and research. Increasingly, they are expected also to contribute to local and global economies by commercializing research and spurring innovation, while addressing grand social challenges like climate mitigation and social disparities. Arguably, universities have come to occupy a strategic place in reshaping society by circulating research-based knowledge through teaching, professional expertise, and other forms of dissemination. That position makes universities important to study. In this book, we draw on the intellectual resources of science and technology studies (STS) to understand continuities and discontinuities in the making and circulation of knowledge, emerging from changes in the relationship between universities and society. Furthermore, we see the study of universities as vital to addressing unexamined assumptions in STS about this relationship as well as the ways in which it may be governed.

Universities are among the most successful enterprises in modern society with stunning growth. They educate an increasingly large proportion of the population in most countries (Frank and Gabler, 2006; Frank and Meyer, 2007), house a huge share of the world's growing research activities, employ large workforces, provide expertise to most other institutions in wider society, influence the public, receive large economic resources, and maintain archives of the world's privileged knowledge. Thus, universities are complex public spaces with a multitude of activities and actors with varying degrees of access that we need to comprehend in order to understand modern societies. Indeed, it is the growing significance and power of universities that have led many others increasingly to seek control over universities' rapidly expanding resources.

To deal with those challenges and waves of social change, universities have been radically and repeatedly transformed. Above all, they have grown and at the same time been re-organized, while the conditions they offer to faculty and students have been altered substantially. A previous President of 
Cornell University has described this transformation in positive terms: 'From modest beginnings over nine hundred years ago, it [the university] has become the quiet but decisive catalyst in modern society, the factor essential to its effective functioning and well-being' (Rhodes, 2001: xi). In contrast, critical voices complain that universities have been corrupted and demolished (e.g., Collini, 2017; Connell, 2019; Donoghue, 2008; Evans, 2004; Fleming, 2021; Halffman and Radder, 2015).

The voices of celebration and outrage agree that by now three longstanding characteristics of universities, the traditional boundaries between universities and wider society, academic freedom, and their epistemic authority all have been altered significantly. By epistemic authority we refer to the multiple ways of asserting, managing, defending, thwarting, circulating, and negotiating knowledge claims, perhaps the defining feature of academic work. Those three activities together are central to this book: how are they being invoked strategically and challenged, by whom, for what ends, as the ecologies of universities are changing fundamentally? We also ask about the relationship between new kinds of order and governance, plus the new success strategies brought by an increasing number of administrators, and the older academic ways of evaluating quality and building consensus, along with making knowledge through teaching and research. Has one obliterated the other, or do they coexist like the dual organizations found in some colonized societies where the outsiders believe they are in control and know how to deal effectively with the local leaders, never realizing that there is an entirely different set of social processes at work when the colonizers are not looking, including different leaders (Ortiz 1965, 1969). Have the old ways been reduced to the margins where they survive and morph into new niche ecologies?

Our attention is on variation within and between universities. We have chosen two such places to explore the local practices, the Norwegian University of Science and Technology (NTNU) and the University of California at Los Angeles (UCLA). We do not see uniformity, whether it be general neoliberal ideas or their translation into entrepreneurial, bureaucratic, and corporate practices, but we do see temporary assemblages of them. We examine that variation and find patterns. As cultures undergo massive change, the participants sometimes decide what to preserve and what to change while exploring the possibilities of changing their ecologies, too. We ask what kinds of experimental changes can be observed on campuses and what sorts of long-term adaptations already have been made. We argue that each of the two universities that we study are buffeted by the same extramural forces with many of the same intramural challenges. However, each currently shows a very different mix of strategic responses. We ask about what that variation signals for the future of universities and what immediate tasks lie ahead.

The book builds upon 25 years of scholarship investigating the nature of the widely acknowledged, massive transformation of universities. We engage with the ongoing claims of greatness and desecration, as well as the explanations 
of why the changes have happened by analyzing what is happening in universities now, given that those changes began about 50 years ago. We do this by looking closely at everyday practices in two universities; both are subject to many of the same forces, but each is engaging with them selectively in complex ways. Understanding the variations between and within them will enable us to evaluate the existing scholarship on the changes, clarifying both what has happened and what might come next. Our goal in this book is to show how our universities are unsustainable and identify solutions.

In the substantial literature that critically discusses how universities have changed, particularly during the last three decades, a widely shared figuration is 'the neoliberal university'. It is characterized by increased privatization and interventions in the organization of academic activities, inspired by discourses emerging from business and mainstream economics to implement auditing systems, efficiency measures, new financial management practices, costcutting, and so-called excellence measures. Critics argue that these changes undermine traditional democratic practices and academic freedom while employment becomes increasingly precarious and academic work is repetitively intensified (Collini, 2017; Connell, 2019; Fleming, 2021; Gill, 2014).

While these critical contributions are important, we want to explore the widespread idea of 'The Neoliberal University' as a singular phenomenon, to be understood as the outcome of a globalized political economy. Of course, there is considerable isomorphism (DiMaggio and Powell, 1983) among universities. Largely, they engage with the same tasks, are subject to the same discourses about excellence and public goods and learn from each other with respect to organizational change. Therefore, the label 'university' is meaningful when navigating the terrains of research and higher education across countries and cultures. According to DiMaggio and Powell, isomorphism is produced through processes of coercion, such as government policies and regulations, mimesis - copying practices from other universities - and norms, setting professional standards for administrative work. Such processes are clearly present in the development of universities, which we analyze primarily in Chapter 2 but also in other chapters. At the same time, as we also show that when we look more closely, there are striking and important variations that indicate countervailing forces to those that drive isomorphism, as well as significant differences in the political-economic contexts and academic traditions of universities.

Many scholars have highlighted such diversity, pointing to the importance of local and national influences (Berman and Paradeise, 2016; Hüther and Krücken, 2016), and contrasting American, European, and Asian specificities (Scott 2017). Paradeise and Thoenig (2013) find that universities may pursue quite different strategies in the face of expectations regarding excellence and reputation, due to diverging views related to what universities ought to achieve. In this book, we focus on differences regarding political-economic contexts, as well as the diversity of epistemic politics and epistemic practices 
that shape, but also emerge from everyday university activities. Such diversity may also create distinct and significant differences within any one university. This variation needs to be considered when developing strategies to challenge neoliberal transformations. We want to contribute to such endeavors. Related to these concerns, we want to emphasize enactments of academic freedom that are embedded in common academic practices related to research, teaching, and outreach. We think that such enactments are important, but frequently overlooked sources of power and resilience. They differ considerably, not the least within the universities, but they are vital parts of the strategic possibilities of faculty and students to shape university politics in the face of various neoliberal interventions. On the other hand, academic life is definitely not without frictions, harassment, and conflicts. These are prominent issues in the book, in addition to the political-economic and cultural concerns in which universities are situated.

At the center of our concerns is the everyday life in university departments, with a focus on enactments of epistemic politics and epistemic practices, where there is tension among the many ways of making, transforming, and circulating important knowledge. Departments constitute the turbulent spaces where university work in all its diversities is conducted. It is in this space that the core tasks of teaching, research, and engagement with society are performed, as well as all the invisible work of planning, communicating, and debating that is a precondition of performing the core tasks. Moreover, the related social interaction is shaped by distinct traditions, routines, and conventions, which means that departments may offer rather different conditions for the conduct of academic work. It is also important that they decisively influence processes of hiring and promotions. While the basic activities of university departments are the same, they are performed and experienced differently. We are exploring some of the underlying dynamics of these activities in the book, particularly in Chapters 3 and 4. This involves analysis of how relevant actors - university leadership, heads of departments, faculty, administrative staff, and students - mediate and enact the multivocal, potentially contradictory concerns that are raised in university settings. Such concerns include funding practices, research excellence initiatives and measures, teaching methods, service work, and learning strategies. We also explore engagements with important social challenges, such as climate change, environmental degradation, innovation, economic inequalities, health, and issues related to gender, class, ethnicity, racialization, sexuality, and other ways of differentiating and dividing people and communities.

We believe our concerns have become even more pressing after the Covid-19 pandemic made universities worldwide close the activities that involved physical interaction, replacing them with online, mediated interaction. Overnight, faculty, staff and students were forced to turn to digital tools to continue to do their work from home. At the same time, the pandemic rendered more visible some of the faultlines that have resulted from the neoliberal policies in 
many countries (Traweek, 2000). An important example is how the pandemic has demonstrated the financial risks involved when universities depend on tuition and other student fees as a major source of income. Another alarming issue is the layoffs that reveal how university employees increasingly have been subjected to precarious conditions of work. Recovery from the pandemic will require a lot of effort; overcoming neoliberal policies that intensified during the pandemic even more so. We intend this book as an intervention in the debates that must emerge, scholarly as well as politically.

\section{Disciplining Universities to Provide Public Goods}

In general, universities have been established to provide public goods through teaching and research. We may question to what extent the contributions of universities benefit the public, but we cannot understand university-society relations without considering the expectations from policymakers, industry, social movements, and so on, of those social benefits. These expectations are the girders of public policies, the enrollment of students, endowments, and initiatives from other stakeholders. They are also, as we shall see, important drivers of the transformations of universities initiated by university leadership. Thus, we consider universities as subjected to disciplining measures to ensure that expectations are met. However, we need to analyze what these measures are and what they amount to in different contexts, as well as what kind of disciplining is threatened and inflicted for non-compliance. We cannot assume the disciplining practices to be similar everywhere.

This view of universities as disciplined is grounded in historical analysis. According to William Clark (2008), the so-called modern research university emerged in Germany in the late 16th century and onwards as a response to a long series of disciplining interventions by the Prussian government. That government began to see universities as a resource on par with the mining industry; what mattered was to make universities realize their potential for supplying goods. The resulting interventions seems strange today, such as visitations to the universities, but some are well known, such as the bureaucratic tools for suitably reporting activities to the authorities. University policy changes in the late 20th and the beginning of the 21 st century followed the same path as 17th-century Germany, but on a more comprehensive and intensive scale. Björn Wittrock (1993) argues that the expansion of research and later the emergence of mass universities through growth in the number of students have been the main transformative forces. The more recent expectations of universities to provide new kinds of public and private goods, such as innovation, spin-offs, and engagements with a variety of social actors, represent yet another set of drivers of change. The instruments of governance and the actors involved in the processes of transformation seem to vary from country to country, but neoliberal visions of effectiveness and efficiency have been widely shared and implemented through new management regimes and 
audit systems (Strathern, 2000), albeit with distinct local adaptations. These adaptations are of particular interest to us.

A main articulation of neoliberal policies is the so-called New Public Management (NPM) policy, a set of ideas to transform the public sector including universities - to make such institutions more efficient and publicly accountable. Hood (1995) claims that NPM is based on seven doctrines. They include the belief in the benefits from implementing private sector-style management, more emphasis on visible, intervening top management, the use of explicit, formal, measurable standards and measures of performance, along with greater emphasis on 'output controls' (p. 96). Similar change doctrines may be found in other manifestations of neoliberalism. For example, NPM is not explicitly referred to in US university discourses, even if most of the measures have been implemented, if modified substantially by the use of corporate software management systems developed in the 1990s. Such policies represent efforts to transform universities into organizational actors (Krücken and Meier, 2006; Whitley, 2008, 2012). The resulting shifts have contributed to the significant expansion and empowerment of university leadership and administration and an increasing public focus on their strategic navigation of external and internal relations. It has also led to quite fundamental changes in the organization of universities, with growing emphasis on practices where instructions flow from university leadership arenas, at the expense of traditionally dominant collegial practices. We may recognize many of aspects of public policies that are described by Brunsson and Sahlin-Andersson (2000) as efforts to construct organizations, which include the construction of boundaries, specialness, hierarchy, and rationality.

However, this does not mean that universities have become homogeneous actors, with departments and research units always acting in step and in line with the grand strategic plans of the institutions. Rather, as we show in Chapter 3, teaching and research, including the writing of research grant funding applications, remains largely at the discretion of faculty. Universities may appear as organizational actors, but with respect to vital practices they are not. This book demonstrates the complexity that still reigns, and which make universities more like what Brunsson and Sahlin-Andersson call arenas 'incomplete' organizations (p. 734). Arenas are characterized by its members and what they do 'are legitimately guided by external interests, values, norms, and standards'. Thus, 'members perform their task relatively free from control by the local leadership'. If we consider universities as arenas, we see them as controlled to a considerable extent by transnational disciplinary norms and standards, not the immediate institutional goals. However, local context and academic cultures are at least as important.

Musselin (2007) claims that universities are different from other organizations. 'In few other workplaces, is it as frequent to ignore what colleagues next door are doing and observe so little influence of the activities of those colleagues on one's own tasks' (p. 70). She also notes how difficult 
it is to describe teaching and research in a concrete, simple, and meaningful manner. Moreover, the relationship between effort and outcome is unclear. These difficulties provide some protection against management of these activities. However, Musselin's arguments about the specificities of universities does not mean that their practices are similar, independent of local context. Rather, she identifies some of the forces that counter isomorphic pressure, emphasizing the complexity of teaching and research. Musselin's observations are intricately linked to a diversity of enactments of academic freedom and the importance of self-management among faculty. Disciplining efforts notwithstanding, the arena character of universities has helped faculty to retain considerable autonomy in their organization of their everyday work. Indeed, it is that autonomy that the new managerial systems try to contain. We discuss this more in greater detail in the book, particularly in Chapter 3, where we also investigate preconditions of academic freedom and how it relates to collegial relations.

In this book, we are concerned with collegial organizing rather than organization, following developments in organization studies (Czarniawska, 2008). We use the gerund form to emphasize the processual and specific features of collegial configurations, assignments, assessments, and decisions. Many studies of higher education, as well as critical university studies, along with public discourse uses the terms bureaucracy, corporation, capitalism, and entrepreneurship as singular generics, which of course they are not, in practice. Many employ the singular generics to suggest some sort of abstraction without clarification; others use them paradoxically to invoke as wide a range of meanings as possible to not limit their claims. Both singular generics and polysemy indicate that epistemic politics are at work. Neither vague abstraction nor polysemy is useful for us. In this book we are arguing that understanding specificities, locations, and practices matter so that we then can identify more fundamental patterns. The processes we find at work are domesticating, co-morphing, meshworking, and faultlines, all of which suggest strategic action to locate new solutions by domesticating and co-morphing of extramural models, demands, and challenges, along with the meshworking of managing everyday practices, while working within and around the concealed but powerful faultlines. Therefore, we are using gerunds, not singular generics.

These are important epistemic distinctions that also imply the application of constructivist perspectives to inquire, for example, into how collegial relations are made, enacted, and imbued with meaning. Collegial organizing represents a way of performing work that involves a considerable degree of self-management and providing for local decision-making. Such collegial principles are often believed to have been predominant at universities in the past, but the principles are seldom specified in any detail. Rather, they are usually implied with reference to both meritocracy and collegiality, ideally characterized by decision-making based on scholarly criteria, in which all faculty participate. In addition, according to Sahlin and Eriksson-Zetterquist 
(2016), there should be some degree of internal representative democracy to cater for larger issues, with participation also from students and administrative employees.

However, as we show in Chapter 3, collegial forms of organizing are not necessarily democratic, even if they in principle allow for such ways of making decisions. Moreover, collegial relationships may well be hierarchical, biased, and involve conflicts, confrontation, harassment, domination, and subordination. Academic life may consist of power games and struggles over positions and reputation (Bourdieu, 1988). We want to contribute to further debates about the features of collegial organizing and what it will take to improve such practices to avoid the counter-productive struggles many academics experience because of dysfunctional collegial organizing. Sahlin and Eriksson-Zetterquist point out that collegial systems at universities increasingly are intersected by practices such as the setting of goals through strategy plans, new standards intended to regulate work and performance measurements, delimiting autonomy. Whether based on NPM or other corporate ideas about governance, these organizing efforts intend to change academic work. For example, they may encroach upon the collegial systems, reducing decision-making discretion among faculty and even removing internal councils with participation of faculty and students. However, as we show in Chapter 2, neoliberal ideas of transformation may be mediated differently in different situations and in different discursive contexts. In this chapter, we explore some of the contingencies of neoliberal policies that may allow for diversity with respect to these epistemic politics and epistemic practices.

Neoliberal policies have clearly been intended as measures to discipline, and sometimes punish universities into supplying public, as well as private goods more extensively and more efficiently. Thus, such policies are coproduced with ideas of the kinds of goods that universities are expected to provide. These ideas can be a mixed bag of concerns. For example, the research policy of the European Union presently calls for so-called Responsible Research and Innovation (RRI). RRI represents requirements that span research ethics, gender balance among researchers, open science, and engagement with the public. The RRI policy expects research to be guided by social problems and the anticipated future needs of industry (Stilgoe, Owen and Macnaghten, 2013). Thus, RRI measures make researchers - and by implication universities - responsible for producing social goods in particular ways that clearly go beyond the traditional practices of universities. RRI may not be effective in influencing academic work (Åm, 2019). However, it serves as a reminder of the need to investigate empirically how universities engage with the making of public goods and what role social responsibility concerns play in their epistemic politics. What expectations do universities face with respect to the kind of public goods they should provide, how they should be provided, and with what qualities? In short, how do universities - or rather, their leadership, 
including their boards - articulate their societal obligations, their responsibilities toward society?

To emphasize the pluralities and heterogeneities of institutions of higher education and research and to identify spaces of action, we have chosen to limit the scope of our empirical analysis by focusing on the juxtaposition of two large, public universities in two national contexts: the Norwegian University of Science and Technology (NTNU) and UCLA. What we offer is a multi-sited analysis, not a comparative study or a single-site ethnography. According to Marcus (1995), using a multi-sited approach means 'to examine the circulation of cultural meanings, identities and objects in diffuse timespace' (p. 96). We have chosen to base this book on our studies of these two universities because they exhibit, between and within them, considerable diversity regarding practices and discourses, as well as the political economies in which they are embedded. We think that it will be helpful to explore and juxtapose these diversities through a multi-sited analysis to understand key features of how universities are constituted in the current context of neoliberal policies and ideology and how universities may enact relations with larger society. We return to related methodological issues at the end of this chapter, where we also provide a brief description of the universities.

Both UCLA and NTNU are large public universities in the formal sense of state ownership, questing for excellence within the locally very prosperous political economies of California and Norway. However, NTNU is nearly completely funded by the government and there is no tuition. UCLA, on the other hand, depends substantially on student fees, donor endowments, and payments for its services, including research and campus-based events. In Norway, the government actively develops policies and initiatives to shape the institutions of research and higher education. The US national government has little direct engagement with universities and the State of California is far less active than the Norwegian government. There is still considerable isomorphism between NTNU and UCLA, but the different political-economic contexts allow us to investigate the implications of these differences for both isomorphism and diversity.

\section{Universities, the Public Good, and Their Quests for Excellence}

In the previous section, we argued that universities are disciplined to produce public and possibly also private goods. This raises questions about the sources and the instruments of this disciplining. We address these questions at some length in Chapter 2, while also considering how NTNU and UCLA process expectations from policymakers and other stakeholders. Some clues regarding the latter issue are found in the isomorphism of public statements that we observe from universities worldwide about their goals. The wording may differ, but the ambitions and the promissory discourses seem without 
limits. For example, the University of Edinburgh's vision is 'To recruit and develop the world's most promising students and most outstanding staff and be a truly global university benefiting society as a whole.' Similar ambitions are expressed by Cornell University in its statement that it

aspires to be the exemplary comprehensive research university for the 21 st century. Faculty, staff and students thrive at Cornell because of its unparalleled combination of quality and breadth; its open, collaborative and innovative culture; its founding commitment to diversity and inclusion; its vibrant rural and urban campuses; and its land-grant legacy of public engagement.

In a more compact statement from another continent, we learn that 'Xiamen University Malaysia aspires to become a university with a distinct global outlook, featuring first-class teaching and research, and embracing cultural diversity.'

Such 'vision statements' and the usually accompanying 'mission declarations' tend to be variations of a trope that highlights a social mission of 'questing excellence' while also pursuing social relevance in research and teaching. Implicitly, this suggests that the trope expresses how universities aim to provide the best possible public goods without any need to prioritize or consider interferences between various kinds of goods. Clearly, in practice this is problematic. For example, Craig Calhoun (2006) argues that we may unpack the abstract idea of the demanded and promised public goods by juxtaposing two key words: 'excellence' - related to the quality of research and teaching - and 'accessibility' that refers to the possibility of acquiring higher education. Both terms are ideological, and Calhoun claims that there is an intensified tension between them. We have looked for such tensions and their consequences.

A radically improved access to higher education has been vital to the emergence of so-called knowledge societies, which depend on mass education, a defining feature of contemporary universities. It is the radical growth in the number of graduates that has created the required labor force. However, accessibility is a relative characteristic, since admission criteria varies hugely between universities. These differences are caused by the perception that some universities and some forms of education are more 'excellent' and thus more valuable than others. They are seen to provide better opportunities in the labor market, which allow them to restrict access. In this way, a focus on excellence creates social inequalities in the labor force of university graduates. According to Calhoun, this dynamic of 'excellence' and restricted access reveals a tension between a public good - mass higher education - and a private good - the labor market situation of the graduates. Moreover, mass education and the consequent wide distribution of scientific and scholarly knowledge in society challenge universities' claim of being excellent. Such 
an elitist phenomenon as excellence can only be attributed to a small number of universities, since only a few can be at the top of rankings. Thus, 'excellence' cannot be a normal feature of higher education; however, the word is a normal feature of universities' discourses, regardless of their resources and achievements. Thus, as a point of departure, we assume that 'excellence' is a performative term with diverse effects.

The widespread reference to 'excellence' in present-day university discourses, such as the vision statements quoted above, confirm that this concept and its various interpretations require critical analysis. What does it mean to feature 'excellence' as a characteristic of the way universities provide public goods? Arguably, the word signifies a striving for quality, but in a vague manner because quality is a relative term requiring explanation and criteria. Weingart and Massen (2007: 95) claim that this vagueness has made the concept widely used because it allows the unification of different interests and positions regarding university policy.

What makes it ['excellence'] so efficient as a political instrument is that excellence has no content: it is neither true or false. Like other political technologies, 'excellence' presents itself as emphatically non-ideological which is precisely why it is so hard to contest or challenge. Could any 'reasonable person' be opposed to raising standards or enhancing quality?

Recent literature on university development exemplifies such diversity, at least with respect to the prescriptions of what should be done to become 'excellent'. One example is Crow and Dabar's (2015) manifesto of what they call 'The New American University'. The book is based on their engagement in the transformation of Arizona State University (ASU). Their main emphasis is on extending students' access to university education as the primary public good. They claim that ASU has contributed to this goal by pledging to accept all applicants. Crow and Dabar also argue that universities should adapt to and interact more with their surrounding communities to provide public goods beyond teaching and research. This requires that social relevance becomes an important feature of research and teaching, claiming that this concern is not constraining these activities in any important way. Implicitly, they reject the tensions described by Calhoun. Another example is John Aubrey Douglass (2016) who proposes that university leadership should prioritize improving and extending the provision of public goods and more visibly demonstrate their university's usefulness to society. This means to go beyond a narrow focus on excellence. Douglass suggests addressing 18 additional policy areas and practices (p. 43), to become what he designates as 'the new flagship university'.

A common argument in the debates about how universities are changing is that universities must become entrepreneurial, leading to 'diversified university income, strengthened steering capacity, extended development periphery, 
stimulated academic heartland, and embracing entrepreneurial culture' (Clark, 2004: 1). Burton R. Clark, a long-time contributor to the literature on higher education, maintains that the combination of insufficient and unsteady funding from the government, along with the marketable potential of universities, drives the emergence of what he calls academic entrepreneurship. Such entrepreneurship, he argues, requires strategic determination and competence, not just from university leadership, but from a broad layer of the employees. Thus, collegial and corporative leadership practices must be joined in some way. This development is necessary, Clark claims, for a university to be able to improve its scientific activities and the employees' belief in its ability to prevail. We analyze consequences of university entrepreneurialism, which is an important articulation of neoliberal ideas and policies.

In such discourses about change, it is claimed that collegial academic practices must be governed or disciplined from 'above' to achieve success. The above-mentioned books are normative, and we mention them here as exemplars of the ideas about universities needing to transform to achieve 'excellence'. The resulting menu of disciplining measures is comprehensive, but the items are hardly surprising. It comprises financial incentives, new standards, digitalization, auditing through numerical performance indicators, assessments of departments and individuals, and the exercise of organizational authority by strengthening the chain of command. Arguably, such measures are the girders of most neoliberal university policies and the widespread quest for excellence. However, 'menu' is a key word to us; the choice of items is the most important.

We study how NTNU and UCLA leadership selects and shapes available tools to quest excellence, whatever that word is taken to mean. Chapter 2 analyzes what 'excellence' signifies as a discursive construct when employed at the two universities. To what extent is it used, to what ends, and with what effects? We believe these questions are important to ask because we expect that neoliberal ideas and excellence measures can be articulated, developed, and implemented rather differently. 'Excellence' may be morphed, remorphed, contested, resisted, and ignored in the face of the strategic as well as the mundane work that needs to be done at a university. Consequently, we also study NTNU and UCLA's discourses about the public goods they aim to provide, and the role given to concerns about excellence. What is presented as important public goods, why are they important, and how are they to be provided? Are there considerations regarding the relationship between public and private benefits?

Vision and mission statements may give some clues about these issues, but only in a very abstract sense. In the book, we are interested in how research and higher education policies articulate more concrete expectations of public and private goods, how NTNU and UCLA appropriate such discourses, and how outside expectations eventually are enacted through epistemic politics and epistemic practices on campus. It is particularly important to study 
the presence and absence of reflections about private goods as indications of the ways in which the two universities are integrated in their national and transnational political economies. Clearly, there are private benefits from having a university degree. Innovations may contribute wealth to a few people. Expectations that new knowledge shall be commercialized may profoundly affect both teaching and research. To what extent are private goods presented as public goods?

At the same time, inspired by Bear and Mathur (2015), we suspect that the articulated discourses about public goods are bureaucratic utopias, that they are expressions of hopes rather than manageable goals. Presumably, the conduct of political and economic university-society relationships is lubricated by highflying, 'hairy' goals that invite positive anticipations among policymakers, funding agencies, alumni, and other stakeholders that are needed for funding and support. Such utopian thinking may lead to the construction of what we call the imagined university (Anderson, 1983; Sørensen, 2019). The imagined university is a discursive construct, a negotiated framework of responsibilities and benefits that are expected to flow between the university and stakeholders, including the public and the state. We are interested in deconstructing such constructs. The books by Crow and Dabar, Douglass, and Clark present distinct imagined universities. Their ideas reflect assumptions about effective governance and institutional governability and about a disciplined, flexible, and obliging university culture. In the book, we explore aspects of 'the imagined university' as it is constructed at NTNU as well as at UCLA and its role in the local epistemic politics. We ask about effects on the performance of university leadership, the conduct of collegial organizing as well as the day-to-day exercise of research, teaching, and engagement with society with an emphasis on epistemic practices.

\section{Navigating the Field of University Studies}

There is a large and growing literature on universities, some of which we already have mentioned. To further explain our intentions and situate our book in the context of other scholarship, we shall briefly comment on some aspects of what may be called university studies. Quite a lot of this literature is critical of current developments, either because universities are seen not to realize their assumed potential as efficient providers of public goods as we discussed above (Clark, 2004; Crow and Dabars, 2015; Douglass, 2016), or because they are considered to be transformed in harmful ways through interventions emanating from transnational and local political economies. The latter point of view has been articulated forcefully through so-called critical university studies, a term coined by Jeffrey J. Williams (2012; 2016). This set of contributions criticizes the tendencies to corporatize higher education and to commercialize research; it represents, above all, a counterpoint to the idea of 'the entrepreneurial university' (Clark 2004). Some lament what 
they see as policies leading to a general decline of universities, the quality of scholarship, and the position of faculty (e.g., Readings, 1996; Donoghue, 2008; Evans 2004). Collini (2017) focuses particularly on the increased social inequality resulting from steeply rising costs of study. Fleming (2021) paints a particularly bleak picture of current developments, in Australia and the UK, describing 'how universities die'. Halffman and Radder (2015) and Connell (2019) add arguments for radical changes to these critical assessments.

A key concept in critical university studies is 'academic capitalism'. It has been given different interpretations. Hackett (1990) uses the concept to describe developments within the life sciences, emphasizing the enhanced role of entrepreneurship, the amassing of resources, the increased anomie, and the growing alienation of subordinate scientists from the means of producing scientific results. Slaughter and Rhodes (2004: 11) understand academic capitalism 'as the pursuit of market and marketlike activities to generate external revenues'; they argue the need to analyze the blurring of boundaries among markets, states, and higher education. Thus, the concept of academic capitalism designates efforts to produce a shift in the regime of making and teaching knowledge that pushes universities away from producing the traditional public goods of teaching and scholarship, instead increasingly requiring them to serve markets and market-related networks (Berman, 2011; Newfield, 2016). This includes engagement in new forms of circulating knowledge and the development of new institutions that align actors within universities, industry, and public administration.

According to Slaughter and Rhodes, those practices link the institutions, faculty, administration, academic professionals, and students to the so-called knowledge economy. They see academic capitalism, at least as it is enacted by US universities, as intimately linked to new ways of making money from knowledge as well as from students. This means that, increasingly, universities are governed by market concerns. Slaughter and Rhodes argue that such governance leads to an intellectual decline because the quality of research and teaching is reduced. Instead of serving the public good by broadly fueling human and cultural growth, the focus is on providing private goods, such as new commercialized technologies and workforces adapted to local labor markets. Burrows (2012) concludes that in such ways, academic value is monetized.

Clark's (2004) call for universities to become entrepreneurial may be interpreted as a call for academic capitalism; this is a needed response to increasingly uncertain public funding and a growing salability of university services. The relative reduction of public funding necessitates efforts to achieve 'diversified university income, strengthened steering capacity, extended developmental periphery, stimulated academic heartland, and embracing entrepreneurial culture' (p. 1). Münch (2014), who uses 'academic capitalism' in his study of the situation in Germany, sees entrepreneurship and university governance from a somewhat different perspective, focusing on bureaucratization, 
the construction of incentives, and the distribution of public funding. We address the paradox of demands for entrepreneurship from those working within highly structured complex organizational framework of universities.

At the core of the changes is the massively increased use of standardized, quantified indicators of so-called 'productivity' and 'quality'. The ways those indicators are generated has become a field of its own with large debates and changes over time, although its concepts, methods, and assumptions are not widely understood. Nonetheless, they form the basis of assessment of reputation and rankings; in some countries, they are used to decide the distribution of resources. The growing use of such 'metrics' also seem to intensify the competition among universities as well as among faculty within universities. This, argues Münch, leads to 'over-investment in currently profitable research, over-researching of topical themes, and under-investment in highrisk research that takes place outside large groups' (p. 253). Resources are concentrated among a few, extraordinarily rich locations, which is a threat to the overall quality of academic inquiry.

Critical University Studies and the work that underpin concepts such as academic capitalism are mainly using a bird's eye perspective. In this way, they provide a generalized description of how universities change and some insights into forceful ideologies and regimes of transformation, along with calls for resistance. In this book, we are aware of these bird's eye perspectives, but our main interest is to pursue a frog's eye point of view; we highlight epistemic politics and epistemic practices as they are enacted in the everyday life of the two universities we study. The metaphors are apt: both birds and frogs are considered 'indicator species': miners carried canaries to provide an early warning of carbon monoxide and frogs are among the first to be affected by water pollution. When doing research academics are very sensitive to changing 'ground states' and also quite good at designing and adapting strategies when research conditions change. We want to study what that strategic game playing in the context of rapidly changing ecologies does to teaching, research, and practitioners, as well as the public policies and financial pressures that universities encounter.

In line with this, we want to add that it is important to avoid an a priori labeling of the collegial system as the benign part of university organization and seeing policymakers and university leadership as mainly a source of trouble. The literature reviewed above tends not to engage in indepth analysis of the collegial practices and the varied pattern of interferences that may emerge from the interaction of university leadership, university administration, and faculty. This implies a too positive view of collegial organizing, which we challenge. The interaction of the different traditions of university organizing with local, national, and transnational actors is another source of interference. It would be a mistake to reduce this complexity just to the two variables of neoliberal influences and academic capitalism. Faculty may still engage with local communities, NGOs, or for that matter industry, without 
complying with the notions of excellence, competition, and commercialization advocated by university leaders (Connell, 2019). This needs to be empirically examined.

\section{Reconfiguring University Studies}

A main strand of university studies research frames universities mainly as institutions of higher education, looking into organizational, managerial, regulatory, and historical issues, as well as studying problems such as social inequality, diversity, career access, student satisfaction, and so on (Gumport, 2007; Daenekindt and Huisman, 2020). Such research has produced important insights, mostly with respect to specific aspects of higher education, its performances, and its effects on future careers of students. However, this strand of university studies tends to neglect what universities engage in besides teaching, such as research and interactions with industry, government, and civil society. Consequently, it does not address the multi-sited, multidimensional epistemic practices that are important to this book and which we discuss, particularly in Chapters 4 and 5.

On the other hand, with a focus on academic work, Musselin $(2008 ; 2011)$ argues that most studies of academics are research centered and a-contextual, neglecting temporality, location, and institutional belonging, such as being part of higher education. Furthermore, she makes the point that

academic work must be studied as a whole, rather than split into different, independent functions. But we lack studies that consider academic activities in different institutional situations or of different status and at different career stages and also explain the variations and the role and scope of faculty discretion.

(2011: 432)

Hackett (1990) observes that the culture of science is different from academic culture, pointing to the need to include, not the least, the context of universities in science studies research.

This book may be read as a response to these calls. We address practices related to higher education, academic work, and research to contribute to multiple fields of inquiry. In doing this, we engage primarily with STS, which is an intellectual home for both of us and which offers perspectives and concepts that have been vital to our work and which we use throughout this book. STS has contributed a host of insights into the diversity of practices of making, assessing, and deploying scientific and scholarly knowledge. However, the main area of concern of STS has been the making of knowledge in scholarly settings, especially among scientists and engineers. For example, the tradition of laboratory studies has not given much mention of institutional contexts or the role of students; an exception is Traweek's work on physicists 
(Knorr-Cetina 1995; Traweek, 1988, 2005). This book is intended as a contribution to expand the scope of STS by examining in a comprehensive way the epistemic politics and practices in the context of the two universities we explore. The importance of this is emphasized by Godin and Gingras' (2000) observation that universities remain at the center of the research systems of many countries: other important knowledge-making actors, such as hospitals, industrial companies, and governmental agencies, among many others, tend to be strongly linked to universities.

At the same time, universities provide society with graduates who have acquired a multitude of disciplinary and professional forms of knowledge. There is little doubt that higher education is the main device for bringing research findings into society. This points to the importance of studying the interaction of teaching and research as a primary set of epistemic practices in order to better understand how this machinery works. The Humboldtian idea of the modern university was based on a rather vague idea of a unity of teaching and research, and the slogan of research-based teaching has widely served as an icon of what it means to be a university. However, its practical implications are notoriously vague. This has also clouded important aspects of university-society relationships that we want to address (see Chapter 5).

Research on university-industry relations argues that contributing to socalled innovation has become a third main mission of universities, in addition to teaching and research. In this context, some authors emphasize how students may be carriers of knowledge that could be a basis for innovation.

Students are also potential inventors. They represent a dynamic circuit of 'human capital' in academic research groups, as opposed to more static industrial laboratories and research institutes. ... the turnover of students insures the primacy of the university as a source of innovation.

(Etzkowitz and Leydesdorff, 2000: 117-118)

This view represents an approach to the issue of research-based teaching that turns the issue on its head, arguing for the need to focus on teaching-based research. In a field like high energy physics (HEP), up to two-thirds of the postdoctoral researchers leave for other fields, industry, and government agencies, making that traffic in post-docs a significant feature of HEP basic research. Those scientists, having spent up to 15 years being educated as researchers, migrate to other arenas with knowledge-making ideas, techniques, and strategies (Traweek, 1988, 2005).

Overall, we want to explore universities as mediators between researchbased knowledge making and society, analyzing its role in the relationship between research and the wider culture. There is little doubt that many faculty members and students regularly engage with a host of social actors or stakeholders, including politicians, public administration, industrial companies, NGOs, and local and national publics. This engagement takes many 
forms, which includes but also goes beyond the usually focused universityindustry collaboration. We study this. Arguably, from a science-society perspective, scientific knowledge is assembled, assessed, and made relevant by going through a series of interactions and transformations between researchers and non-researchers where issues may be raised, social implications explored, and value preferences articulated in both directions (Latour 2004). Nowotny, Scott, and Gibbons (2001) propose the metaphor of the agora to designate places where researchers and the public may meet and engage in dialogue. We see universities as such spaces, but this role is not acknowledged by Nowotny and colleagues, nor by most scholars concerned with science-society relations. Policymakers as well as science studies scholars tend to pay less attention to universities, possibly because there has been too little reflection about the wide-ranging consequences of the emergence of the so-called mass university and the enormous growth of university graduates.

During the last 50 years, as previously noted, higher education has been transformed from an elitist phenomenon to a rather mundane activity. In an OECD report, State of Higher Education 2015-16, Saricco (2017: 4) succinctly describes the situation:

Based on current patterns of graduation, an average of $35 \%$ of today's young people across OECD countries is expected to graduate from tertiary education at least once before the age of 30 , some $57 \%$ are expected to enter a bachelor's degree or equivalent program, and $22 \%$ are expected to enter a master's degree or equivalent program over their lifetime.

Arguably, university teaching is the main site for the public's engagement with research.

To pursue all these issues, this book examines universities as public spaces hosting a variety of activities that engage an array of social actors, guided by and contributing to their epistemic politics and resulting in a diversity of epistemic practices. We study how universities themselves design and perform their public role and how it is enacted and how conscious and concerned university actors are of their strategic position as mediators between research and society. What strategies do they employ to enhance this mediation work, and in what arenas are these strategies employed?

\section{Homeward Bound: Engaging Ethnographically with Universities}

Overall, universities get a lot of attention in many countries, politically, economically, socially, culturally, and intellectually. However, as we have seen, scholarly work on universities tends to focus on historical developments and structural issues, paying less attention to the everyday aspects of academic work, such as the relationship between teaching and research, the conduct 
of collegiality, the local competition for resources, the interaction between professors and university leadership, and the performance of diverse kinds of outreach, much less the vast amount of epistemic activity. In line with this, we share Hugh Gusterson's (2017) concern that scholars such as anthropologists should engage much more with what he calls 'homework', doing critical ethnography of universities, the places where we work. His call for such reflexive investigation is particularly relevant to our own scholarly communities: those engaging with science and technology studies, as well as gender studies; in addition, Knut is a sociologist, plus Sharon is an anthropologist and historian. As already mentioned, we see STS research as particularly relevant and useful for studying universities in the context of science-society relationships and with a focus on mundane practices of knowledge making, the performance of hierarchy, university management, and enactments of excellence. This includes the investigation of how universities and their disciplines and professions deal with epistemic authority, claims to be the main suppliers of knowledge within boundaries that increasingly are challenged by outside voices. They demand a much greater emphasis on social relevance, interdisciplinarity, and wider participation in the making of authoritative knowledge.

This book presents results from our many years of 'homework' in order to offer a comprehensive and original account of university life and its relevant contexts, with a particular interest in exploring how university actors of many arenas respond to expectations that they provide an increasing amount of what are considered public goods (which clearly includes private benefits, such as economic advantages from having a university degree or profiting from utilizing research to make innovations). The public mission of universities is no longer limited to educating social elites or governmental and military executives. How is the new and extended mission understood and enacted as the political economies of research and higher education are transformed in an economically, politically, and socially fluctuating world? What are the consequences of such extended transformations for the organization of universities, for the everyday life of professors, staff, and students, and for the interaction between universities and their surrounding communities and societies? We study how this has unfolded at NTNU and UCLA. More generally, we are describing large, ongoing changes in very established cultural practices in academia, primarily at universities with a strong focus on research.

Our homework incorporates two people's reflections on their decades in academia as academics. It is a multi-sited, multi-method ethnography employing participant-observer research methods, conducted by two very experienced practitioners. We address situated knowledge-making practices in two different, but related ecologies. These ecologies include relations with other universities, participation in workshops and conferences, posts in professional societies, funding agencies and official committees, and so on. During our long academic careers both of us have studied many kinds of knowledge-making sites around the world. We now bring that experience to 
the understanding of universities, including our own. In doing such work we invoke decades of STS scholarship on epistemological, theoretical, methodological reflexivity (e.g., Ashmore, 1989; Fortun and Fortun, 2005; Martin, 2009; Vertesi, 2020). That means we carefully consider the challenges and opportunities of investigating our own epistemological, theoretical, and methodological investments as we study those of others.

The conventional scholarly writing template still requires the use of third person singular verbs in the passive voice and often elides plurals and specificities into singular generic nouns, while also avoiding the first and second person pronouns. In fact, Routledge wanted us to revise the blurb on this book to meet those conventions. In agreeing to do so we decided to include this paragraph within the book. During the 1980s (Haraway, 1988; Harding, 2009; Hartsock, 1987) many scholars began to criticize that 'voice from nowhere' and demand a 'strong objectivity' that situates the knowledge makers and their knowledge-making practices in the text so as to provide readers with a far more empirically accurate account of the processes for making the knowledge work being presented, as well as the subjects of inquiry and the knowledge makers' interactions. Another group working during the 1980s criticized the intellectual complacency of only questioning the assumptions embedded in the epistemic practices of those being studied while neglecting to do the same about our own work (Ashmore, 1989; Ashmore, Wooffitt, and Harding, 1994). In the subsequent 35 years many scholars in STS, Feminist Science Studies, and Social Epistemology Studies, among others, have refined and expanded upon those initial interventions. In this book (and our other scholarly work) we contribute to those accomplishments; we skip the passive 'voice from nowhere' and shift from generics to gerunds as we both study and engage in stronger objectivity and reflexive epistemic practices, locating the multiple power relations in those activities. The conventional template strategically masks the power that enables it, rendering it far from objective.

Our book is not a study of anyone's individual psychology, cognition, agency, or decision-making. Our perspective is anthropological and sociological, with a focus on the interactions within communities about their knowledge-making practices as their ecologies are changing. Our primary research method is ethnographic participant-observer fieldwork. It is a common exercise in such research to note what those we are studying make of us and what use they make of us as participants in their world, whether short or long term. Understanding the ways in which they make sense of us enables us to learn more about their ways of making sense more generally. The exercise also enables us to calibrate how our presence might affect their actions and hence our observations.

How do we study our own ecologies and our own place in them? The first requirement of autoethnography is an intense awareness of and engagement with all the epistemological, theoretical, methodological challenges. In some 
parts of this book we describe our own experience with the issues at hand, including our interactions with others and what they make of us. These are not comments from diaries or memoirs. The incidents we invoke were selected because they are representative of many similar comments from colleagues, revealing customary practices, the normal ways to accomplish the expected tasks, plus how people learn those customs and change them. We cite our own experiences because they are examples of the changes in forms of knowledge making in universities, graduate education pedagogies, faculty positions, research practices, and evaluations, as well as subject formation during the course of a career. By citing our own experience as representative, rather than unique, we then are charged with specifying that representativeness. By not citing the experiences of others we avoid causing any potential harm to our research subjects.

Autoethnographies and studies of reflexivity both challenge and examine the assumptions embedded in the categories of objectivity and subjectivity, as well as how those categories are cast as oppositional and what is enforced by those categorical imperatives. Put differently, we are invoking a research and interpretive strategy outside the dominant epistemology of the ecologies we are studying. We practice multiple forms of parallax, two of us gaining depth of understanding by each of us examining our subject in at least two ways. This strategy is more complex than most, but not unlike how many academics navigate their multiple, everyday worlds, making sense as they observe, reflect, share, and learn. Academics have been well educated into the canons of how research should be done; we can recite and perform the manual: do not use the first person singular and always use the passive voice, present the research report as if there had been no researchers present. The conventional rules require adherence to the canon of hierarchical binaries. The costs of being seen as a body of emotions is well known. Our presentation is a break with that orthodoxy.

Autoethnography is not the opposite of the dominant epistemology or its absence. However, it challenges binaries, categorical imperatives, and canons. Yes, it is a rational, reasonable, complex, and subtle form of knowledge production, but generated within a different epistemology, outside most conventional academic canons. We ask you to pay attention to our epistemology as we enact it through our writing and acknowledge it as different, without relentlessly invoking the obvious: it may not be yours. To label it as subjective misses several fundamental points that should be noted. Keeping this in mind, the reader should also take notice that the autoethnographic voices frequently are intersected with other kinds of voices emerging from our and other people's research, policy documents, other kinds of research we have been conducting or been part of, which include interviews, oral histories and surveys, newspaper articles, and so on. These sources are acknowledged when we make explicit use of them. Furthermore, we depart from a flat ontology. This means that we do not invoke hierarchies in the form of levels, but as 
actors and actions articulating power, strong or less strong, moving analytically between them without constraints.

A stunningly large amount of information about how universities work has not been described. That means access to that knowledge is restricted and provided only to those invited to participate in each domain. In this book we are being explicit about important matters often kept tacit, or not discussed in public. From many studies of tacit knowledge, we know that it is not open to revision, while explicit knowledge is open to debate. One demands subordination without reflection or commentary; the other requires incessant scrutiny and debate, sometimes with formulaic tools. We are required to learn both, along with how they must be compartmentalized. By discussing these tacit practices explicitly, we are violating that taboo. Proctor and Schiebinger (2008) have argued that the pervasive silence about everyday activities and studiously maintained ignorance about others is perhaps the most important feature of academia. Understanding why that silence and ignorance has persisted among intellectuals and how that silence and ignorance is required, accepted, and exploited in universities is an important feature of our analysis.

\section{Questing, Resisting, and Re-morphing Excellence}

Initially, our idea was to focus on our universities' - or, rather, their leadership's - quests for excellence, often expressed in terms of rankings and similar numerical indicators. In our experience, the searches for excellence increasingly have permeated university discourses, morphing the idea of what universities are for into a frame of competitive sports with endless performance and game statistics. This perspective is still a girder of the book, but we also see the need to highlight how 'excellence' has been both re-morphed and resisted through the mundane, but foundational everyday activities of students and faculty. The quests for excellence are important, but not unequivocally so. The two universities we study are intersected by a multitude of concerns and powers that interferes to provide some space for contingency. We are not dismissing or devaluing complaints about austerity politics and neoliberal managerialism; our focus is on how in practice austerity and managerialism are shaping and being reshaped by those sharing the same ecology.

We explore these complex spaces through a focus on locating the making and distribution of knowledge in a diversity of university arenas and by studying the rhizomic practices that result in the complex roots of different academic and policy-making performances that help to constitute our two universities. As we shall see repeatedly in several chapters, this is embedded in a conglomerate of ideas and policies that have shaped and are shaping modern universities as drivers of isomorphism, meaning, at minimum, that universities are expected to be providers of so-called public goods. The book is a study of two universities. As mentioned earlier, this is not a comparative 
analysis where we look at differences and similarities with respect to organization, funding, management, and so on. Instead, it is a multi-sited, multimethod, and autoethnographic analysis that explores and conceptualizes epistemic politics and epistemic practices - primarily at NTNU and UCLA, but also drawing on experience from other places. Thus, it is not a study of NTNU and UCLA as organizations, but an effort to contribute concepts and observations we hope will be useful to understand the making, morphing and mobility of knowledge in universities more broadly.

We draw upon a distinctive body of evidence. We have studied the daily changing narratives, promissory discourses, allocations of resources, divisions of labor, decision-making, subject formations, embodied performances, career histories, and transmissions of knowledge across generations, plus the construction and maintenance of intellectual, social, material, and cultural infrastructures in local and distributed communities of practitioners. That intensive field research is combined with our extensive experience at other sites. We have been registered, affiliated with, and employed at 12 public and private universities in Japan, Norway, Sweden, the UK, and the United States. We have conducted research on three continents and presented our work in 13 countries around the world. In addition, as students, faculty members, and dissertation advisers we have engaged in transdisciplinary research and teaching with a wide range of scholarly fields, departments, programs, institutes, professional societies, funding agencies, and publishing venues, both conventional and not: Anthropology, Communication Studies, Education, Gender Studies, History, Information Studies, Japan Studies, Science, Technology, and Society (STS), Sociology, and Urban Studies. Our former advisees are academics in those fields in universities around the world and hold leadership positions in international professional societies. In addition, we have engaged with interdisciplinary projects at many sites, including years of field research among astronomers, engineers, and physicists based at universities around the world. Of course, we are aware of the reflexive epistemological and ontological ironies invoked by our career-long ethnographic case studies of knowledge-making practices; those interpretive strategies are part of our research and ground our claims to be constituting new approaches to understanding knowledge making through our teaching, research, and civil society engagements.

\section{Intervening}

We particularly intend this book to be an intervention in STS debates (this intervention is also relevant with respect to higher education studies). Our arguments challenge current STS positions regarding the making of knowledge, most importantly by focusing on universities as public spaces where knowledge is made, remade and flows through the complex interaction of many actors, not the least faculty and students, as well as a rapidly growing 
managerial class who often circulate to other teaching, research, and cultural organizations. Rather than analyzing either research (like STS does) or teaching (that higher education studies do), we attend simultaneously to research, teaching, governance, and society. By integrating these concerns, we produce a more robust and comprehensive account of the making and traveling of academic knowledge, which has considerable scholarly as well as political potential. We do this by proposing a new set of arguments and queries where we highlight intersections of research, teaching, innovation, and dissemination:

First, we study the intersection of teaching, research, governance, and society in university life as characterized by meshworking, the making of lateral intersectional webs of relationships without homologous units, juxtaposed with the hierarchically structured relations that we also analyze. There is significant variation among those webs and not all are built at the edges of the mainstream. Furthermore, we show that participation in meshworks and dominant hierarchical structures are not mutually exclusive. We find that those participating in meshworks may have multiple approaches, just as in hierarchical relations. We investigate how people are building and using diverse webs of relationships structured in diverse ways to sustain and develop strategies for knowledge making, resources, teaching, outreach, and narratives about their practices. This adds important novel issues that STS should pursue.

Second, we observe how neoliberal ideas dominate present academic knowledge narratives. This happens as policymakers and university leaders domesticate these ideas by mainstreaming them into decision-making at all levels. Many of those practices are adapted from corporate management strategies of earlier decades. We find that they do this using the labels of excellence, social responsibility, innovation, and diversity, along with a growing set of standardized metrics to measure the new phenomena and a regulatory apparatus to enforce compliance, generating new forms of academic subjectivity, no longer focused on quality, ethics, originality, and knowing. Thus, we find that the making and mobility of academic knowledge is situated in and shaped by an ecology that STS has only barely addressed.

Third, in the book, we present universities as situated co-morphings of leadership, administration, funding, teaching, research, outreach, services, reviews, assessments, change management, campus development, interdisciplinarity, and innovation. 'Co-morphing' extends the ideas of co-construction, so widespread in STS, while avoiding the implied expectations related to stability and closure. In particular, we intervene in STS by showing how this continual co-morphing involves knowledge-mixing activities in the interaction of university and wider society. We show how this means to facilitate the embedding of academic knowledge in society by pursuing social relevance, for example, by mobilizing emergent, liminal knowledge, remixed to address social needs and innovation. 
Finally, we analyze the rupture between the old resources and strategies for quality, morality, originality, and knowing with the new goals of excellence, responsibility, innovation, and diversity that have occurred along old and newly discovered faultlines. This has not been sufficiently acknowledged in STS. Above all, we find that knowledge-making infrastructures and modes of inquiry, as well as formations of expertise and experts, usually co-morph with new kinds of political economies of universities. Those changes do not happen everywhere at once, just as industrialism and the emergence of research universities, beginning in England and Germany, respectively over 200 years ago, continue now in some places. Like then, the expansion of information and service-based political economies has brought new practices in knowledge making, standardization, surveillance, cultural production, public spaces, affect, subject formation, and expertise. We argue that older lines of inquiry and boundaries around the territories of expertise are rupturing massively. To understand how universities operate in the knowledge ecology, we focus on the current strategies for coping with the exposed faultlines, which are concentrated on making excellence, responsibility, innovation, and diversity with potentially serious consequences for the meshworking needed to provide for robust and relevant knowledge.

Our argument is distinctive because we attend simultaneously to research, teaching, governance, and society. We analyze these interactions, drawing on a co-morphing framework in which each transforms the other, generating new patterns of knowledge making. No part controls the others. Moreover, we revise the conventional interpretation of universities by attending to a wide array of everyday university practices and the meshworks tying those activities to others around the world. We also consider how university leadership, through neoliberal framings, domesticates the emerging global issues into local concerns. In addition, we study universities in the plural while also emphasizing the considerable diversity within each institution. We explore the details of everyday life in the two universities to explore the confining effects of the excellence regimes on faculty and students. We show how these effects originate from the rupture of faultlines in the cultural and political economies of universities. While acknowledging such negative effects of neoliberal practices, we go beyond this diagnosis by considering a host of other features to offer a comprehensive understanding of late modern universities.

There are obvious limitations to our project. We study two relatively affluent universities situated in rich countries. Both of us have privileged positions that have allowed us to move around and explore the academic world. We are certain that readers will take our 'standpoints' and 'situated knowledges' practices into consideration and reflect on our choices of topics and perspectives, as we certainly do (Collins, 1997; Haraway, 1988; Harding, 2009; Hartsock, 1987; Wylie, 2012). Although scholars have engaged in multimethod, multi-sited, and reflexive ethnographic research, along with studies of how researchers studying other people deal with those encounters ethically 
and epistemologically for at least 50 years, we hope this example will provide new strategies for those designing such research.

Universities are important and influential institutions. They exercise epistemic authority and power, practices which need to be scrutinized and understood. At the same time, they are places of work for the administrators, faculty, and students that are shaped and reshaped by political and economic forces, often in harmful or misguided ways. The insights that critical university studies offer, as we briefly reviewed above, are important as interpretative tools and to allow for critical distance. However, as mentioned, we also think that it is vital to identify possibilities of resistance and transformation that can be used to pave the way for what Connell (2019) calls for 'the good university', or rather - in line with her arguments - good universities. We return to such issues in the conclusion.

\section{Locating Two Universities}

In this chapter we have presented the aims of the book and its key concepts. We also provided an overview of relevant scholarly work in STS and higher education studies, showing how our work intervenes in those debates, in particular through its focus on universities as public places of knowledge mixing. Now we briefly introduce our field sites, the Norwegian University of Science and Technology (NTNU) and UCLA. We situate them in their local historical political economies as well as in the global arena of universities. We outline why we are examining them together.

\section{NTNU}

The history of higher education in Norway is usually considered as beginning with the establishment of the University of Oslo in 1810. Before that, Norwegians who wanted to study had to go to the University of Copenhagen. Since then, there has been a huge growth in the number of higher education institutions. As of 2021, Norway has ten universities, six regional colleges, and five scientific colleges, in addition to many private institutions. NTNU is located in Trondheim, the third largest city in Norway with a population of 208,000 . Norway's population totals 5.3 million. Trondheim was established around 997 and the first grammar school began in the 11th century. The first academic institution in the city, the Royal Norwegian Society for Science and Letters, was founded in 1760. NTNU was established in 1996 through a merger of the Norwegian Institute of Technology and the University College of Arts and Sciences. The institute of technology had been founded in 1910; the university college grew out of a college for higher education of teachers that was started in 1923. Both these institutions were placed in Trondheim after long debates in the parliament. The decisions reflect a historically strong political emphasis on regional concerns to locate institutions of higher education outside Oslo, the capital of Norway. In 
2016, NTNU merged with three regional colleges to become the largest university in Norway with around 40,000 students. The merger happened as a response to a government policy of restructuring, or rather, centralizing higher education in Norway to reduce the number of institutions.

The presence of the government in the organization of the university, its siting, its mergers, and its activities is paralleled by the presence of the government in the funding of NTNU. In 2019, its total income was around 1 billion US dollars: 72 percent of this came as a grant from the Ministry of Education and Research, calculated through a budget model that is applied to all higher education institutions in Norway (more details about the model in Chapter 2). An additional 12 percent came from grants from the state funded Research Council of Norway and 2 percent were grants from the EU's Horizon 2020 program. Income from industry and private gifts constituted 2.5 percent. Most of the rest of the revenues came from other government institutions.

Thus, NTNU is nearly fully funded from the government and subordinate institutions. This legitimizes extensive policy interventions, which we describe in greater detail in the next chapter. The income from private industry is too modest to modify these interventions. Typically, NTNU holds only a few shares in private companies, valued at around $\$ 6$ million. ${ }^{1}$ This low figure also reflects that Norwegian universities are not allowed to accumulate funds or endowments other than for covering recorded financial obligations in coming years. Compared to universities in many other countries, the financial situation of Norwegian universities has been relatively good. During the last decade there has been an overall increase in the funding from the government. In the wake of the pandemic, it is noticeable that the dominant role of the government as funder of higher education largely has protected Norwegian universities from economic repercussions. In fact, the pandemic has provided a surplus due to reduced travel costs and similar expenses.

The Ministry of Education and Research does not only govern NTNU through goals and measurements as we would expect from an NPM regime. It has also established three directorates that intervene more directly with NTNU and the other universities. ${ }^{2}$ One of these is Diku - the Norwegian Agency for International Cooperation and Quality Enhancement in Higher Education. It was set up to manage several national and international incentive schemes 'to promote quality' in universities and colleges and has 130 employees. It also produces policies and programs that are intended to promote development and innovation in education and to encourage international cooperation and the development of digital learning methods. ${ }^{3}$

A second and more prominent one is NOKUT - the Norwegian Agency for Quality Assurance in Education. It was established to attend to accreditation and review of quality assurance systems in higher education.

NOKUT's work is intended to contribute to society at large having confidence in Norwegian higher education ... The agency has 150 employees 
and is organized in four departments and a communication unit. During a two-year period, around 900 experts are involved in various evaluation and accreditation processes under the auspices of NOKUT. ${ }^{4}$

These processes are intended to force the universities to implement comprehensive quality control regimes with respect to teaching, requiring a lot of bureaucratic efforts, including reports about teaching practices and achievements. NOKUT is one of the foremost drivers behind the bureaucratization of Norwegian universities.

The third directorate is Unit - the Norwegian Directorate for ICT and Joint Services in Higher Education and Research. It provides governance of and access to shared ICT services to all institutions of higher education. This includes the Norwegian Universities and Colleges Admission Service (NUCAS), which coordinates the admission to ordinary undergraduate study programs at all universities, university colleges, and some private university colleges in Norway. Unit also manages the government's program for open access publishing. ${ }^{5}$ In 2021, DIKU and Unit were merged.

\section{UCLA}

The University of California at Berkeley (UCB) was founded in 1868. Over 50 years later UCB gained a 'southern branch' in Los Angeles which in turn conferred its first doctoral degree in 1936; both were accomplished against the strong opposition of UCB. By 1951 UCLA and UCB had equal legal status within the new University of California system, but UCB still had far greater prestige and continued to regard itself as 'first among equals' as UC acquired more campuses. In 2018 the Times Higher Education World University Rankings named UCLA as the best public university in the United States. How did that happen? The answer lies in the changing global political economy and the changing place of California and Los Angeles in that ecology, as well as what rankings mean, a topic we will rejoin in Chapter 2.

Before significant numbers of settlers began to arrive in 1770, the land now called California was home to at least 300,000 indigenous people divided into about 70 distinct groups. They have endured successive waves of occupation by Russia, Spain, Mexico, and the United States. Those four different kinds of colonization were genocidal for the native people; only 50,000 remained in 1855 and half that were alive in 1910. There were about 8,000 non-native people in California by 1840, increasing in ten years to about 110,000, primarily due to the Gold Rush. As early as the 1830s settlers had begun to discuss independence from the four colonial powers; briefly there was a California Republic. (Three other US states once were independent countries: Hawaii, Texas, and Vermont.) However, following battles in southern California, the land was annexed to the United States in 1848 and that territory officially became a state in 1850 . 
During the Spanish and Mexican occupations of California there were many primary and secondary schools throughout the region. Universal elementary school education was required in the state's first constitution. The first colleges were established in 1851 and 1852: California Wesleyan College (later University of the Pacific), Santa Clara College (now Santa Clara University), and Mills College (the oldest women's college west of the Mississippi River). The United States Congress passed the Morrill Act of 1862 (supplemented in 1890) to support the development of colleges that would focus on agricultural and engineering studies; the support came from the sale of federally owned land, hence the label 'land-grant colleges'. ${ }^{6}$ Often the land on which the universities were built was unceded Native American territory, as is the case with UCLA. Each state could make its own plans for the schools. The California Assembly Bill No. 583, introduced March 5, 1868, serves as the charter of the University of California which was located in Berkeley, near the San Francisco Bay. By 1870 the state had 560,000 inhabitants. ${ }^{7}$

The first California State Normal School (for teachers) was established in 1881 at San Jose, near San Francisco; their southern branch opened 1882 in Los Angeles. In 1919 the same school was renamed the southern branch of the University of California; in the intervening 40 years Los Angeles had become slightly larger than San Francisco, having grown from about 11,000 to 575,000 residents, inaugurating many decades of rapid expansion, slowed only by the pandemic. By 1900 California had become the largest US oil producing state; the biggest oil fields were in the Los Angeles area. A film entertainment industry grew rapidly from 1910, but agriculture remained a prominent economic sector for the region.

Until WWII the economy was focused on agriculture, oil extraction, and oil refining with additional investments in the banking and filmmaking industries. Conflict with Japan led to the rapid development of a vast military infrastructure, from the expansion of ports in Oakland, Long Beach, San Diego, and San Pedro, to the establishment of a powerful aircraft and weapons industry, as well as the transformation of the film industry into a huge public war information apparatus that also developed complex communication technologies. The western frontier had become a powerful war engine. That wartime infrastructure and its attendant expertise, combined with investments in the Cold War of 1950-1990, became, during the second half of the 20th century, the foundation for the vast expansion of the aerospace, entertainment, and computing industries. In 1960 the state had 10 million residents. By 2000 California had become by far the most populous of the United States; in 2020 the population was nearly 40 million, larger than Poland, Canada, Morocco, Saudi Arabia, Uzbekistan, Peru, Malaysia, and so on. Similarly, the economy is the largest in the US and if it were an independent nation-state, it would be fifth largest in the world. The Los Angeles region has become a 'technopolis' - an urban area with an economy centered on five high technology industries: primarily aerospace, automobiles, entertainment, oil, and 
higher education. Los Angeles County now has about 10 million inhabitants and the greater five-county region is nearly double that. The city includes a microcosm of the world's population; over 40 percent were born outside the US, 10 percent higher than any city in US history. It also has the largest diasporic indigenous population of any US city. Children in the public schools speak nearly 100 languages at home. Only about 40 percent of UCLA students are monolingual.

All those historical forces (imperialisms, colonialisms, migrations, wars, demographic growth, wealth, etc.) also have transformed California's universities. Arguing that the expanding California economy required a highly educated workforce and anticipating that a large proportion of the baby boomers would attend college, Clark Kerr led the development of a 'Master Plan for Higher Education in California' that was adopted in 1960 (revised in 1987 and most recently reviewed in 2010), all founded as 'land-grant' educational institutions. They planned an unusual and enduring three-tiered system, along with defining the relations among the tiers: the California Community Colleges, the California State Universities, and the University of California. ${ }^{8}$ Note that all 'residents' of the State of California pay reduced fees; residency is achieved by living continuously in the state for one year. Non-residents (those from outside California, whether another part of the US or another country) pay fees equivalent to attending a private school. Those non-resident fees have become a significant income source for all three tiers of the California public higher education system, especially as the 'brand name' of some campuses have attracted many students from afar. At UCLA 25 percent of the undergraduates are not residents of California.

According to the Master Plan for Higher Education in California the California Community Colleges have two goals: prepare students to transfer to a California State University campus or a University of California campus and generate a skilled workforce for the local industries and government agencies. The California State University campuses are to prepare students to participate in California's culture and economy. The mission of the University of California is to provide long-term societal benefits. In addition to their allotments from the California state and local governments, as well as fees from students, campuses in all three systems raise money from private sources for endowments. Their additional sources of funding include research grants from government agencies at all levels, non-governmental organizations, and private sources. The 115 Community Colleges get 100 percent of their funds from state and local governments. The 23 California State Universities get about 80 percent of their expenses from the state. The ten UC campuses get approximately 7-30 percent of their expenses from the State of California. Of the UC campuses UCLA raises the most funds from donors and fees for various services, so the allotment from the state is a very small part of its budget. ${ }^{9}$ 
The California Community College (CCC) system now has 115 campuses, 2.1 million students, and over 90,000 faculty and staff members; it is the largest higher education system in the US. They accept any resident (including newcomers from anywhere in the world) able to benefit from instruction. They offer many courses preparing students of any age for occupations in local industries, from coding and aircraft maintenance to scriptwriting and computer graphics. In addition, these colleges offer the first two years of a university education, preparing students to transfer to the California State Universities (CSU) and the University of California (UC) campuses. Many students prefer to attend a community college and then transfer to $\mathrm{CSU}$ or UC for the final two years. Community colleges receive funding from both the state government and the local governments where they are located. ${ }^{10}$

The CSU system currently has 23 campuses, about 485,000 students, and nearly 56,000 faculty and staff members. It is the largest four-year higher education system in the US. They accept the top 33.3 percent of California high school graduates. In addition to Bachelor's degrees, CSUs offer Master's degrees in 258 subjects, and doctoral degrees in 69 topics, primarily in the education, engineering, and health fields. The CSU campuses are similar to most of the land-grant state universities of the US east coast and mid-west. Undergraduate students submit one application to all CSU campuses and list their campus preferences; CSU Long Beach has the most demand, so they are the most selective. ${ }^{11}$

In 1945 the UC system included the Berkeley campus and its southern branch in Los Angeles, along with some agricultural research institutions. Importantly it also had incorporated some WWII weapons laboratories, including the 'Manhattan Project' site in Los Alamos, New Mexico, where the nuclear weapons dropped on Hiroshima and Nagasaki were developed. Now UC has ten campuses with over 280,380 students and 227,700 faculty and staff members: Berkeley, Davis, Irvine, Los Angeles, Merced, Riverside, San Diego, San Francisco, Santa Barbara, and Santa Cruz. UC also administers three national laboratories and several agricultural research institutes. UC has over 800 doctoral degree programs. As a system UC accepts the top 12.5 percent of high school graduates.

Undergraduate students submit one application to all UC campuses and list their campus preference. For many years UCLA has received more applications than any other university in the US and it has been the first choice of all UC undergraduate applicants, so UCLA is able to be more selective than the other UC campuses. In 2021 UCLA accepted 10.7 percent of 139,463 undergraduate applicants while UCB accepted 14 percent from 112,820 applicants. From 2014 to 2019 UCLA received $\$ 5.49$ billion in donations. For the last ten years it has received over one billion dollars a year in extramural research support. In 2012 there were only seven other universities in the US with that level of research funding from all sources: UC San 
Diego, UC San Francisco, the Universities of Michigan, Washington, and Wisconsin, plus Duke and Johns Hopkins. In 2019 there were 19; UCB is \#31 nationally in terms of extramural research funding. ${ }^{12}$

There are now 168 public and 136 private universities and colleges in California. Several rank among the top 100 universities in the world. Together they receive extensive extramural research funding, donations, and fees for their services from many sources. They also employ several hundreds of thousands of people. That is, in California higher education is not only seen as a contributor to the knowledge-making economy, it is a powerful economic sector in itself and has been so for at least 50 years. UC announced in January 2021 that it contributed:

roughly $\$ 82$ billion annually to California's economic output. Over a half-million jobs in California - or one in every 45 - are supported by the University, and UC-related spending generates nearly $\$ 12$ billion annually in federal, state and local tax revenues.

UCLA contributes the largest portion of that. ${ }^{13}$ The strongest universities in California became so through their long, very close meshworked relations with industry, the military, and government, surviving together successive turbulent ruptures of California's local and global faultlines (Traweek, 2000). For that reason, UCLA and others like it can stand in sharp contrast to most universities around the world that have been prompted only recently by their national government patrons to participate more fully with the private economic sector; many still struggle with those mandates and opposition remains strong.

Both NTNU and UCLA are situated in rapidly changing political economies that are increasingly wealthy and focused on knowledge production. Universities are an important part of those economies, generating both knowledge and knowledge makers while employing significant workforces. Both universities have shown remarkable resilience and adaptability during all those changes. The reputation of each place has been steadily rising internationally, although neither was particularly well known 50 or 75 years ago (however, NTNU has dropped in the international rankings after the merger in 2016). In all, they have been very well situated to benefit from the emergence of the information based political economy. Both are also actively engaged in new sustainability initiatives, both for their campuses and as sites of innovative research to make the surrounding areas more sustainable. Both have accommodated quickly to the new quantitative measures of campus activities. In that context, people at both sites have shown many signs of the stress correlated with the outward signs of success. Our study of the local, everyday strategies for successful adaptation to the new global ecologies explores the patterns in those strategies. 


\section{A Brief Outline of This Book}

The next chapter focuses on the political-economic contexts of our two universities. We explore how they manage reputation and what reputation means, before we investigate how NTNU and UCLA construct and interpret performance metrics. This includes an analysis of the role of government, which we show is particularly influential in the case of NTNU. Chapter 3 explores enactments of academic freedom and how collegial organizing is circumscribed and shaped by bureaucratization and corporatization. We also study how collegial organizing may be affected by factions and how meritocracy and academic citizenship are important to upholding academic freedom, although in ambiguous ways. We pursue these issues and the effects of local political economies on academic subject formation in Chapter 4. This implies a concern for the diverse ways of structuring university education, in particular the enactment of research training. We also investigate academic assessments and how they may be experienced. Chapter 5 focuses on universities as knowledge utilities and the meshworking activities performed through teaching, innovation, and interdisciplinarity. To analyze university-society relations we study how universities make, assemble, interpret, and share knowledge with other social actors. We emphasize how such meshworking is vital to the influential position of universities as nexuses of knowledge making and social practices.

In the concluding chapter, we address the issue of making universities sustainable. Sustainable development was defined by the Brundtland commission as efforts to meet the needs of the present without compromising the ability of future generations to meet their own needs (Brundtland et al., 1987). In line with this understanding, we apply a comprehensive approach to the challenges involved in developing a sustainable university. First, we notice how the growing focus on global warming and other environmental concerns has forced universities to address their own climate footprints and environmental impact. Second, universities have come under increasing pressure to economize their resources in ways that threaten their relative autonomy, as well as the working conditions of faculty and students, leading to increased precarity and job insecurity. This threatens the social sustainability of universities as academic institutions. Third, universities are a microcosm of the wider society, so universities' ongoing struggles with failures and success in recognizing and addressing longstanding inequities that have saturated their projects of teaching, research, and societal engagements can be instructive. Finally, we emphasize how universities may play a vital role in providing knowledge and assessments that are needed in the sustainability transitions that are called for in wider society; however, more humility and a better understanding of how knowledge moves are needed for this to happen.

Unlike almost most other studies of neoliberal universities and academic capitalism we have situated those transformations and their contradictions 
in the everyday practices of students, faculty members, and university administrators. As academic fieldworkers we examine those ideas and actions ethnographically and reflexively. We set the stage in Chapter 1 by locating our work. First, we investigate two universities, NTNU and UCLA, differently situated in global political economies. We also position our work alongside that conducted in the fields of higher education studies and critical university studies, plus science, technology, and society studies. We map four key concepts to navigate four transformations at work in our universities, addressed in the next four chapters. As we explore the tropes of quality, governance, subjectivity, and mobility, we show the strategic work of domesticating, faultlines, co-morphing, and meshworking.

What seem to be global, abstract, and universal forces are always made local through specific domesticating processes, as we show in Chapter 2 with the strategic management of evaluation metrics and reputations, changing debates about quality into increasingly meticulous measures of certain kinds of productivity and consumption. As ecologies cycle through ruptures and stabilization, governance practices manage the faultines, often through hiding them, recognizable through the incessant work such concealment takes, replacing the governance of universities by academics with university administrators using corporate and bureaucratic tactics, as traced in Chapter 3. In Chapter 4, we show how that radical change in governance has been facilitated and resisted through the performance of different kinds of strategic mutations of academic subjectivity, co-morphing into outliers, cooperative citizens, and authorities, some of whom coalesce into factions, collaborating with the occupying bureaucrats and corporatists. Alongside the increasingly enclosed disciplines we find a dense traffic across the still porous boundaries of departments, universities, and societies, done with meshworking: as described in Chapter 5, many students and faculty members constantly circulate, unmonitored, among disciplines, universities, and sectors of societies, building new commons with webs of relations among and between intra- and extramural worlds. That unmonitored circulation builds new ways of making and teaching knowledge.

In Chapter 6 we first interrogate the compulsory and compulsive corporatizing and bureaucratizing of the last 50 years, along with their obsessive metric monitoring and attendant factionalizing of resources among those in control, showing how the entire apparatus is simultaneously unsustainable and cannot address sustainability. Although the new governors claim they are building cost-effective knowledge productivity with directives, incentives, efficiency, and surveillance, this book shows how they are binding teaching, research, academics, and outreach ever more tightly. The astounding avalanche of corporatizing administrators arriving at the University of California since 1975 (Lu, 2021) and the provocative proliferation of bureaucratic directives at NTNU demonstrate a pathology Bateson first called schismogenesis (1958). In his work on cybernetic epistemology he argued that such positive 
feedback loops will increasingly intensify until the entire process collapses (Bateson, 1972/2000, 1979). Sustainability in universities and global ecologies both urgently require that we cut those loops. Fortunately, sustainable, alternative knowledge-making ecologies do exist in the margins of universities, as we outline what is to be done.

\section{Notes}

1 Arsrapport (2019-2020), www.ntnu.no/documents/10137/1279418159/NTNU_ aarsrapport_bokmaal_2019.pdf/d9e76719-afa7-18e2-dd8b-0ab363f090d9?t= 1589976134263 (accessed August 10, 2020).

2 During 2021, these directorates will be merged and supposedly streamlined.

3 https://diku.no/en/about-diku (accessed February 8, 2021).

4 www.nokut.no/en/about-nokut/ (accessed May 10, 2020).

5 www.unit.no/en (accessed February 8, 2021).

6 For Morrill Acts of 1862 (7 U.S.C. $\$ 301$ ) and 1890 (26 Stat. 417, 7 U.S.C. § 321) see the US Library of Congress, 'Primary Documents in American History' at www. loc.gov/rr/program/bib/ourdocs/morrill.html (accessed March 19, 2019). For links to additional Congressional acts 1887-2003 to support the states in developing colleges see https://en.wikipedia.org/wiki/Morrill_Land-Grant_Acts (accessed March 19, 2019).

7 Among the holdings of the Bancroft Library, University of California, Berkeley. See http://bancroft.berkeley.edu/CalHistory/charter.html (accessed March 19, 2019). The 106 current land grant universities are members of the Association of Public and Land-grant Universities (APLU) www.aplu.org/, www.aplu.org/ members/our-members/ (accessed March 19, 2019). See also Douglass (2000).

8 See http://universityofcalifornia.edu/aboutuc/masterplan.html, www.ucop.edu/acadinit/ mastplan/mp.htm and www.ucop.edu/acadinit/mastplan/masterplanRegentsJan2005_ final.pdf

9 More information is found here: www.cccco.edu/, https://vision.foundationccc.org/, www2.calstate.edu/csu-system/about-the-csu/Pages/mission.aspx, www2.calstate. edu/attend/graduate, and www.ucop.edu/uc-mission/

10 See: www.cccco.edu/, www2.calstate.edu/apply/transfer/Pages/upper-division-transfer. aspx/, and http://admission.universityofcalifornia.edu/transfer/index.html

11 See: www.calstate.edu/, www2.calstate.edu/csu-system/about-the-csu, http:// admission.universityofcalifornia.edu/transfer/index.html, www2.calstate.edu/ attend/graduate, and www.calstate.edu/AS/stats.shtm

12 www.ucop.edu/, www.universityofcalifornia.edu/uc-system, https://graduate.uni versityofcalifornia.edu/admissions/, www.latimes.com/california/story/2021-0719/uc-admissions-new-diversity-record-but-harder-to-get-in, and www.nsf.gov/ statistics/infbrief/nsf14303/. Some think the UCSF and UCB numbers should be combined, but that argument has flaws, given that the two campuses have many similar programs and departments.

13 www.universityofcalifornia.edu/press-room/uc-s-economic-impact-california-hitsnew-high-82b-annually and https://newsroom.ucla.edu/releases/ucla-2020-economicimpact-report-108000-jobs 


\section{Chapter 2}

\section{Disciplining Universities \\ Performance Metrics, Policy Reforms, and Reputations}

\section{Introduction}

Worldwide, universities operate in quite different political economies, which shape their funding and their governance. This is not simply about how the wealth of nations affects university resources. There are many distinct systems of higher education. This reflects different relationships between universities and governments, not least the expectations regarding public goods. Governments may be active in shaping universities or take a more distanced approach; they may be clearly present or nearly absent in the governance of such institutions. They may provide funding in diverse ways. NTNU and UCLA, albeit both public universities in the legal sense, operate in quite different governance contexts as we learnt in the previous chapter. Here, we explore these differences further and how the universities navigate them. At the same time, we want to remember that both universities operate in relatively privileged economic contexts and have clear financial advantages, compared, for example, to universities in Australia and the UK that suffer from severe fiscal austerity measures from their respective governments (Collini, 2017; Fleming 2021).

We argued in Chapter 1 that historically, governments have tried to discipline universities to provide public goods, such as qualified candidates and socially useful research. As we shall discuss in Chapter 3, the disciplining is moderated substantially through ideas of academic freedom that often are supported by law. This limits the extent to which governments in Norway and the US may intervene directly in the content of teaching or research, as well as employment of faculty. Thus, the disciplining tends to be based mainly on indirect measures and general regulations that affect how, for example, NTNU and UCLA organize their operations and develop strategies. To what extent are these universities disciplined to emulate academic capitalism or similar market-like mechanisms? How is the making of public goods assessed, and with what consequences?

Besides their traditional role of providing social elites with status markers, modern universities emerged above all to provide highly qualified graduates 
in the classic professions of law, medicine, and theology, many of whom were employed by the government. When during the 19th and 20th centuries, industry and government increased their demand for competence in engineering, agriculture, forestry, health care, social services, and teaching, these demands tended to be met through the establishment of institutes of technology and colleges focused on agriculture, nursing, teaching, and so on. However, more recently, those kinds of education have become integrated in universities, which also have expanded into the social sciences, economics, and increasingly, new interdisciplinary fields of study. NTNU and UCLA both cater to a broad set of expectations for education and research. In this manner, the two universities have responded to expanded demands for public goods.

When we focus on the political-economic contexts, we study how the contexts are interpreted and acted upon. As suggested in the previous chapter, we expect to observe considerable differences between the strategic practices and measures employed by the leadership of NTNU and UCLA, as well as their related discourses, to accomplish their goals. We are particularly interested in how 'excellence' and reputation are articulated and managed, but also in how public policies are transformed into local practices. How important is the relative presence and absence of governments?

While these contexts are important, universities are also competing nationally and transnationally. In the third section of the chapter, we explore this by showing how NTNU and UCLA react to such competition by trying to improve their reputation through marketing, thus branding the institution. In turn, this raise questions about the role of rankings and metrics, which in Chapter 1 we argued to be vital features of the governance of and competition between universities. However, interestingly, NTNU and UCLA deal with these issues quite differently. We show how these differences are closely related to the political economy of the two institutions, which make them measure and process performances, as well as excellence and reputation, in dissimilar ways. To demonstrate some of the diversity among neoliberal universities, the rest of this chapter analyzes how rankings are used with respect to reputation, and the national and local management of metrics. By what features of the two universities are measured, for what purposes, and with what effects? This includes a more detailed exploration of the excellence practices of NTNU and UCLA and how the two universities consider provision of public goods, with a focus on how the two universities relate to their respective governments and other stakeholders.

Another related and important topic for this chapter is the autonomy of universities. NTNU and UCLA face a host of expectations and promises, regulations, and funding options that are produced through their respective political economies. These forces discipline the universities to provide public, as well as private goods to serve the many stakeholders that articulate such demands, including governments and industry. Some of these 
issues were addressed in Chapter 1 when introducing NTNU and UCLA. Here, we focus on the multitude of disciplining measures shaping the two universities we study, how their leadership interprets and mediates the articulation of the epistemic politics involved, and the emerging epistemic practices. This affects the space of navigation of university leadership. We analyze the effects on faculty and the enactment of academic freedom in the next chapter.

\section{Distinctions: The Role of National Governments in Governing Universities and the New Metric Managerial Models for Universities}

Before proceeding to discuss how NTNU and UCLA are disciplined we need to make three important points: one concerns the different role of national governments for NTNU and UCLA. The second concerns the very different kinds of funding sources that support the two universities. Finally, the metric management systems being used at NTNU and UCLA to allocate and manage resources are distinctive. In what follows we show the implications of those three differences.

NTNU has a very close relationship with its national government. UCLA does not. Historically, national governments have played a key role in the development of universities around the world, but far less so in the US. That difference extends into the present. While most public universities outside the US are funded almost exclusively by their national governments that has not been the case for the leading public universities in the US. Many did receive significant support from state governments, but those revenues began to decline about 50 years ago and the change has accelerated over the last 25 years. Most leading universities in the US, public and private, have a wide array of 'revenue streams'. One significant stream is research funding; that comes from many different national and state government agencies, as well as corporations, and non-profit foundations. Another funding source is student fees for registration, lodging, and board. A third important source is campus events, especially sports. Donor contributions provide another powerful source of funds.

Both NTNU and UCLA are quite dependent on their financial 'stakeholders', which are different in kind. This makes it pertinent to inquire about the autonomy of universities. Whoever funds higher education and research, it is because they expect benefits in return. The development of neoliberal policies, such as metric management models, represents efforts to make universities contribute more public, and increasingly also more private goods within economic and administrative constraints. The biggest change in everyday university governance since the 1970s has been the introduction of metric management practices which are now pervasive. The goal is to make universities contribute more goods for society within carefully monitored 
specific economic and administrative constraints. At NTNU and many other universities the new metric managerial requirements for universities have been imposed by national governments. In US universities those managerial practices have been 'self-imposed' and take another form. The metric managerial models used in US universities typically have been developed by the business sector during the 1980s and 1990s; since then much of the business world has moved in another direction, but the products they developed at the end of the last century were then marketed to universities and various government agencies.

It appears that the New Public Management (NPM) protocols widely used in Europe, the UK, and Australia were initially based upon the business models of the 1970s and 1980s, but then strongly modified by national and international governmental organizations. In the US, the metric management models developed by corporations have been widely adopted 'as is'. While both NTNU and UCLA use metric managerial systems pervasively, they are not the same protocols. In this chapter we explore the implications of these metric management models while keeping in mind their differences which will become apparent at different stages.

Metric management models have both increased and reduced the autonomy of universities (Christensen, 2011; Aberbach and Christensen, 2018). The assessment of autonomy raises complex issues, some related to the balance between autonomy and accountability, contingent on local conditions (Maassen, Gornitzka, and Fumasoli, 2017). That is, the metric management models include both extramural and intramural data. Intramural data can show how various parts of the university are contributing to meeting extramural goals. Extramural resources can be distributed and managed intramurally, but there is extramural accounting surveillance. Much leadership and disciplining in contemporary universities addresses the spreadsheets provided by the metric management systems that all groups have been trained to provide. That is, at the interface of goals and performance are the metric management models.

Assessment practices have always been integral to academic work. Peer review has been the most visible of these, but academics are also readers and writers who continuously evaluate the quality and relevance of scientific and scholarly work. These craft practices are based on qualitative interpretations in the context of specific disciplines and specialties. While the concept of 'peer' allows considerable interpretative flexibility, it still refers to the specialized academic competence usually needed to legitimately assess the quality and relevance of a given piece of work. Traditionally, this dependence of peer review has limited the assessment and decision-making power of university administrators and policy makers who have lacked the legitimacy needed to evaluate faculty arguments about quality and their performance.

As already indicated, the introduction of NPM and more generally, metric management practices represented efforts to amend this situation. We 
discussed some aspects of NPM in Chapter 1 and its effects on universities. The growing use of NPM policies has meant that universities - above all in Western Europe and Australia - have come to face what Wilsdon et al. (2015) call 'the metric tide' - pervasive quantitative indicator systems based on a growing range of aspects of academic work that are made measurable. To be fair, efforts to develop performance indicators of research institutions and researchers go back at least to the 1960s, when Derek de Solla Price paved the way for launching what later would be called the field of scientometrics (Leydesdorff and Miljojevíc, 2015). Counting publications was one early form of such measurements. Eugene Garfield's development of the Science Citation Index opened new possibilities for using citation counts as an assessment tool. Nevertheless, scientometrics which involves the use of a much wider set of indicators to investigate knowledge-making practices has played a minor role in the metric tide.

Jerry Z. Muller (2018) points to what he calls a metric fixation as typical of neoliberal forms of governance. Its key components are the belief that (p. 18):

a. judgment based on personal competence should be replaced by standardized numerical indicators;

b. making such metrics public (transparent) secures that institutions will do what they are supposed to do (accountability); and

c. people are best motivated by incentives related to measured performance.

Decades of research in many fields has challenged each of those assumptions, but many still believe them.

\section{Corporatizing and Bureaucratizing Are Not in Opposition}

When addressing changes in universities over the last 50 years, critical university studies have been concerned with the development of 'academic capitalism', often described as a corporatizing of universities, inspired by neoliberal ideas that market principles are more efficient than public bureaucracy (Slaughter and Leslie, 1997; Berman, 2011). We use the concept of corporatizing to describe efforts that primarily aim to increase the external funding of a university, aligning the organization to effectively pursue this goal. Usually, this was assumed to involve imitation of industry with an emphasis on clear chains of command with appointed rather than elected leaders and the articulation of highflying mission statements and ambitious goals. UCLA's use of a 'hub and spoke' management model fits into that hierarchical decisionmaking practice, as does their use of corporate metric management protocols. Data are collected at all levels and used by leaders to make decisions about meeting goals defined by stakeholders. Corporatizing also means that impression management is given a lot of attention, such as branding and rankings, 
to which we shall return, together with considerable efforts of university leadership to develop external relations with powerful actors that through financial or other forms of contributions may strengthen the university. Thus, the activities of university leadership receive much attention, while the efforts of faculty and students remain rather opaque.

In addition, universities may pursue a bureaucratizing strategy as a response to neoliberal governance, such as NPM or other metric management systems. This may seem paradoxical since neoliberalism claims to replace bureaucratic decision making with practices from industry. However, this paradox is endemic to universities, partly because bureaucratic systems are needed to provide accountability and governance. In fact, during the 1960s a quantitative approach to planning, management, and decision making based on military operations research and systems analysis in computing was implemented at Ford Motor Company by Robert S. McNamara. He and his team from Ford then used the same approach to policy analysis and governance at the US Department of Defense as he managed the Vietnam War. Later he did the same as president of the World Bank; he had begun his career as a professor of accounting at Harvard (Rosenzweig, 2010). ${ }^{1}$ That particular approach to management had become very well established in government and industry before it was introduced to governing universities.

We see bureaucratizing in universities as a process with a focus on control and homogeneity, with an increased emphasis on defining standards with respect to decision making in most areas of university activities. Digitization is a crucial tool to implement standards, since this allows the use of an increased number of online forms and templates. Assessment of performances is vital. In this respect, bureaucratizing means increased use of measurements, such as publication indicators, success in gaining external funding, and students' evaluation of teaching. There is also an increased requirement for reports of many kinds, such as documentation of teaching quality. Bureaucratization is also driving a shift from administrative support for faculty to administrative interventions to control faculty.

The two strategies may be combined in distinctive ways when universities domesticate neoliberal ideas, government policies, innovation discourses, and so on. Domestication theory highlights how the use of devices such as technologies or discourses involves work to establish concurrent practices, to interpret them to provide meaning, and social learning for further development of practices and sense-making (Sørensen 2006). For example, we expect neoliberal ideas of 'excellence', 'accountability', 'innovation', and 'diversity' to be transformed, explained, and enacted in particular ways; the question is how. As we indicated in the previous section, such domestication processes are aligned with a growing set of standardized metrics to measure performances and development of regulatory apparatuses meant to enforce compliance. The outcome of these efforts may not be considered successful, 
but we understand them as attempts to generate new forms of academic subjectivity. We return to this in Chapter 4.

\section{Building University Reputations: Branding and Ranking}

NTNU and UCLA face a host of expectations and promises, regulations, and funding options that are produced through their respective political economies and the university administrators. These pressures and opportunities discipline the universities to provide public, as well as private goods to serve the many stakeholders that articulate such demands, including governments and industry. Some of these issues were addressed in Chapter 1 when introducing NTNU and UCLA. One of those required goods has been excellence. Before exploring the mandated quest for excellence at NTNU and UCLA and how the two universities are managed to achieve that goal, we introduce two global practices for defining excellence in universities and what makes them distinctive: branding and ranking.

\section{Branding as a Strategy for Building a Distinctive Reputation}

A basic element of the prevailing neoliberal university discourses is the assumption that universities worldwide increasingly compete with respect to students, faculty, and funding. In short, they supposedly struggle for access to scarce resources, including attention. However, this competition is conceived in quite diverse ways at the two universities we study. UCLA's Academic Plan only indirectly hints at external challenges with expressions like 'enhance our competitiveness' and 'remains competitive in respect to salaries, support, and fellowships'. ${ }^{2}$ NTNU's strategy plan expresses greater and more explicit concern. 'NTNU will experience more intense competition, both in recruiting excellent staff and students and in obtaining research funding. Competition will become increasingly global'. ${ }^{3}$ Such worries have been recurrent in NTNU's strategic deliberations.

The different articulations of the issue of competition may reflect different cultural notions of competition, different standings in the local and global landscapes of higher education, and different modes of thinking about running universities. At the same time, there is considerable isomorphism between NTNU and UCLA in their marketing endeavors. Seemingly with inspiration from industry about how to succeed in achieving public recognition, university leadership in recent years have hired marketing companies to help them gain visibility by branding of the names of the universities. Eventually UCLA established its own office for branding and public relations. How are universities branded and what are the implications of such efforts?

In 2018, NTNU launched a campaign based on the slogan of challenge. The underlying idea was that inventiveness stems from asking critical questions, challenging existing knowledge. In one of the campaign videos, 
the audience is asked to challenge everything, including a series of social issues. The concluding image shows NTNU's motto: 'Knowledge for a better world'. ${ }^{4}$ NTNU has in previous years launched similar campaigns, where the main intention has been to focus public attention on the university. NTNU's reputation is supposed to be built from public recognition of the name of the university, not from professional achievements. A year later, UCLA launched a campaign with the slogan 'Knowledge Solves'. Commenting upon the campaign, Jim Poore, UCLA's Chief Creative Officer, who works in the Strategic Communications Office, launched in 2019, stated that 'UCLA's combination of academic excellence, intellectual curiosity, public mission and location in a global city make it a unique and powerful force in both higher education and society." The campaign clearly aimed at branding UCLA as a name to be recognized by the public, but at the same time, it spoke about the university's academic feats. The branding was based on reputation; not the other way around, as was the case with NTNU.

Still, both campaigns reflect a transnational idea of what universities are and what they are for. Considering the history of universities more broadly, such isomorphism is not surprising. As we briefly argued in Chapter 1, 20th-century developments have changed universities from relatively modest institutions of higher education into large-scale providers of research and knowledge to engage with a wide variety of societal concerns. Branding is supposed to produce an identity that makes that university appear different from all other universities. A comparison between NTNU and UCLA's campaigns suggests that this is difficult. It appears that the most distinguishing feature of a university is its location. Thus, typically, most universities are named to show where they are placed.

At the same time, it is important to observe that the branding efforts were met with considerable head shaking and ridicule on both campuses. For many bragging is a sign of weakness, or worse. At NTNU there was a lot of public criticism and many parodied the slogan, such as 'Challenge the neoliberal university' (Davies, 2020). Quite a few voiced a feeling of shame that academic activities at their university were reduced to such simple slogans. Still, the leadership defended the campaign and the marketing people were happy about the controversy, since it made the campaign more visible. Marketing is all about attention. At UCLA the new branding team focused on redesigning the university logo, specifying the font and exact shades of blue and yellow that must appear in all official communications that use the name of the campus, as well as on copyrighted merchandise that is both widely marketed and overpriced, alongside cheaper, pirated versions. ${ }^{6}$ Like all corporate logos UCLAs are heavily regulated and defended legally; nonetheless, they are quite easy to mock. For example, modified logos are found in the altered UCLA decals pasted on student possessions, caricaturing who owns what. The intensified attention to the UCLA logo as brand began just as the fashion for embracing them abruptly shifted, yet again, among trend setters. NTNU 
has also focused on its logo and changed it a couple of times, without much public attention. It also sells some merchandise with the logo but is not a significant source of income or attention.

NTNU's and UCLA's branding campaigns could be read as efforts to interpret the otherwise unclear slogan of 'excellence', which may not communicate well with the public or even prospective students. Instead, the campaigns focused on general claims about the usefulness of university knowledge for society as an important ingredient of the public goods that universities are supposed to provide. Presumably, 'excellence' works better with policy makers and potential donors, although not without ambivalence. The efforts to brand the universities clearly reflect a market-oriented framing of higher education and they signify that the leadership wants their university to appear as a single organizational actor, a homogeneous entity that may be unified under one banner (Krücken and Meyer, 2006).

Universities' investments in branding raise questions about how the management of reputation relates to the political economy of the institutions. The attention given to university rankings suggests that reputation is important, but in what way? Is it mainly about status? Are rankings considered politically important? To respond to such questions, we need to examine the metric practices of the two universities, including how indicators are employed and for what purposes. It is noticeable that indicators that are used in university rankings go beyond measures of research excellence to include assessments of teaching, diversity of students and faculty, graduation rates, and relative income of graduates. Presumably, university rankings have pushed the development of metric systems to assess universities. However, as we shall see, rankings are not the only driver behind such systems. Governments may be important drivers as well, depending on their role in funding and policymaking.

\section{University Ranking Systems}

As noticed above, university rankings have been an important driver behind the metric tide that has hit universities. In the US, the US News \& World Report has published university rankings since the 1980s, and actors in several other countries have followed suit, such as Germany, UK, Spain and Italy (Usher and Savino, 2007). The initial UK and US rankings had led to greater interest in such rankings both within and beyond national borders by different groups concerned with international higher education and university-based research practices: students deciding which schools to attend, faculty members deciding on their preferences for employment, funders seeking contextual information for the research proposals they receive, and policy makers determining future directions in higher education, locally, nationally, and globally. This attention to global rankings in the UK, Canada, Australia, and the US also correlates with the vast increase in foreign students attending universities in those countries; those students' fees are a particularly important funding resource in the 
budgets of universities and departments that attract such students; each place wants to increase their 'market share' of such students.

There are now three major world university rankings systems; two are based in the UK and one is in China. Each uses different variables and formulas to calibrate their rankings, although all address the amount of research published and the research funding accrued. They vary too in the relative emphasis they place on accumulated, as opposed to current reputation, and whose opinion is consulted, from researchers in the same field to funders, students, corporate employers, and so on. All rank both universities as a whole, as well as subject areas because not all subjects would be equally strong at the same university. Some address clusters of subjects while others focus on specific subject areas and subfields; some take new fields into account and others only include traditional disciplines. Some emphasize STEM subjects. Each publishes a set of reports annually.

The data for the Times Higher Education (THE) World University Rankings were provided by the Quacquarelli Symonds Company 2004-2009 and Thomson Reuters 2010-2013; Elsevier data have been used since 2014. QS World University Rankings (WUR) has operated independently since 2010. The Academic Ranking of World Universities (ARWU), sometimes called the Shanghai Rankings, was launched in 2003 by the Center for WorldClass Universities at Shanghai Jiao Tong University; it is now published by the independent Shanghai Ranking Consultancy. After 2003, the ARWU rankings have been developed to challenge what many perceived to be a bias in the Times rankings toward European and then North American universities. Its focus is on larger universities. Those three groups' annual ranking reports are mentioned in US news media and covered extensively on US campuses, as at UCLA. The information is posted at campus websites, along with the rankings generated by national groups. ${ }^{7}$ In Norway, the rankings are given little if any public mention. University newspapers may mention them, but not always.

There are well-known, fundamental problems with such rankings (e.g., Davies et al., 2021; Espeland and Sauder, 2016; Hicks et al., 2015; van Raan, 2005), but that does not seem to reduce their popularity or their use in allocation of resources. A similar case is the assessment of academic journals through the so-called 'impact factor' which is deeply flawed (Seglen 1997), but frequently invoked as a proxy for the quality of the papers they publish and even used in the assessment of university employees.

Criticism of university rankings and other metric systems abound, and we share these assessments. However, here we focus on the construction of such systems at NTNU and UCLA, their relation to governance, how they are linked to ambitions about excellence, how they are enacted, and how they affect university activities. Such calculation of university reputations offers some degree of transparency, but they are primarily a menu of management tools, which university leadership may choose from and interpret. Rankings 
may be used in different ways, or not at all (Paradaise and Thoenig, 2013). Metrics may also be applied to the assessment of individuals and departments, and various forms of quantitative performance measurements provide for assessment of fulfillment of goals (we return to this issue in Chapters 3 and 4). The current ideas of excellence have been co-morphed with the development of metrics; there is some degree of isomorphism, but also considerable flexibility of interpretation.

We noticed above that the branding-related, neoliberal claim that universities compete transnationally with respect to students, faculty, and resources was explicitly acknowledged at NTNU, but less so at UCLA. Although competition among US universities can be acute, it takes local knowledge to be aware of the competitors that matter. In terms of extramural research funding UCLA primarily competes with the Universities of Michigan, Washington, and Wisconsin, plus UC San Diego and UC San Francisco, although Johns Hopkins far exceeds all the others; Berkeley, Harvard, Yale, and Stanford are not significant competitors. However, UCLA does give much attention to its public rankings relative to UC Berkeley, quite aware that in the national and international public imagination Berkeley's reputation continues to exceed UCLA's. In terms of donor contributions, UCLA might compete with the nearby University of Southern California and Stanford, but as yet the three universities appear to be taping into different wealthy communities, locally, nationally, and internationally. All three universities have successfully used donor funding to launch decades long campaigns to improve their hitherto mediocre reputations; Stanford launched their plans first, in the 1960s and 1970s, followed by UCLA in the 1980s, and then USC in the 1990s. In terms of student recruitment UCLA is extremely successful. UCLA faculty recruitment is successful too, especially relative to all the other UC campuses, but is limited by the comparative high cost of living in Los Angeles, a problem also faced by universities in New York City.

\section{Presences and Absences: Features of the Political Economies of NTNU and UCLA}

Both NTNU and UCLA are public universities. However, this label says little about the actual involvement of government. In principle, governments are sources of funding, but also of policies for higher education and research. Previously, we have argued that, historically, governments have disciplined universities to increase their provision of public goods (Clark 2008). However, when we started to exchange notes about the political economies of our two universities, we observed striking differences that we attribute to the relative presence and absence of governments. Thus, we decided to use the juxtaposition of governments' relative presence and absence to structure our analysis. This does not mean that this is the only significant feature of their political 
economies, but as we shall show, its presence and absence also shape the space of influence for other actors and interests. Moreover, government presence seems to invite certain kinds of bureaucratizing, while its absence seems to encourage certain kinds of corporatizing.

\section{NTNU - a Bureaucratizing University with Government Presence}

Norway definitively provides an example of government presence in the context of universities. After 2000, there has been at least 18 substantial government-induced reforms of higher education in Norway. This includes institutional restructuring of higher education, introduction of the Bologna system of $3+2+3$ years of standardized duration of Bachelor's, Master's, and $\mathrm{PhD}$ degrees respectively, and a new budget system (Sørensen, 2019). In a blog from April 2021, the leadership of the University of Oslo complained that they presently must deal with a new set of government reform suggestions that amounts to 17 initiatives! There is no doubt that the Norwegian government is actively present in higher education. ${ }^{8}$

Relevant White Papers and similar policy documents express clear expectations regarding the production of public goods from universities and colleges. The most recent is the Long-term plan for research and higher education 2019 - 2028 (Meld. St. 4 (2016-2017)). There are three prominent objectives that these institutions should address: strengthened competitiveness and innovation capability for Norway, solving grand societal challenges, and the development of specialist communities of excellent quality (p. 17). The White Paper explains these goals and relates them to politically prioritized research areas, which include ocean-related bio-economy, climate and sustainability concerns, public sector innovation, and so-called enabling technologies. The Norwegian grand challenges are not particularly remarkable and include sustainable development, 'green' transitions, and sustainable welfare. Excellent specialist communities are defined by their ability to develop 'new knowledge, new insight and innovative solutions. They provide high quality education and communicate results from research and development in such a way that the knowledge may be utilized' (p. 27). It should be noticed that the plan does not refer to university rankings or similar metrics. Quality is described in qualitative terms.

However, this does not mean that metrics are not used in the governance of the universities. The award letter that accompanied the government's budget for NTNU for 2020 lists four long-term, main goals for higher education in Norway that NTNU must pursue. First is high quality in teaching and research. The second is that research and education should serve welfare, value creation, and social change. Next there should be good public access to education. Finally, the higher education sector and the research system should be efficient, diverse, and robust. ${ }^{9}$ The letter further states that achievements 
with respect to these goals will be measured by an assessment system decided by the government, using the following set of parameters:

1. 'High quality in education and research' is to be gauged by

a. The share of bachelor candidates completing their degree within the standard time frame (of three years).

b. The share of master's degree students completing their degree within the standard time frame (of two years).

c. The share of $\mathrm{PhD}$ candidates completing their degree within six years.

d. The average number of hours per week full-time students spend studying, measured by a yearly national survey called the Student Barometer.

e. The average score of how students assess the quality of their study program, measured by the Student Barometer.

f. The number of publication points per academic employee, including $\mathrm{PhD}$ candidates. Publication points are measured through a dedicated calculation system utilizing a data base where all employees of institutions of higher education are supposed to register their publications.

g. The value of grants from EC's Horizon 2020 program, per full-time employee doing R\&D.

h. The share of students participating in the Erasmus + exchange program of the EU.

2. 'Research and education should serve welfare, value creation and social change' - this is to be measured by

a. The share of master's degree graduates who are employed in relevant jobs within six months of graduation, calculated from an annual survey of graduates.

b. The income from the Research Council of Norway per academic employee, including $\mathrm{PhD}$ students.

c. Other income from contract research per academic employee, including PhD students.

d. Share of research conducted within science, engineering and medicine.

3. 'Good public access to education' is to be measured by:

a. The number of graduates from programs in teaching and health.

4. 'The efficiency, diversity and robustness of the higher education sector and the research system' is to be measured by:

a. The number of students' credit points produced per academic employee, including $\mathrm{PhD}$ candidates. Students are expected to achieve 60 credit point per year.

b. The share of women in full professor positions.

c. The share of temporary employees among faculty. This share should be low. 
The award letter further refers to a development plan, agreed upon by NTNU and the Ministry. This plan contains five more goals, which specifies the overall sector goals with a view to specific challenges that the Ministry asks NTNU to face. The achievements are to be measured by 24 additional parameters. Thus, NTNU is to be assessed through a government specified metrics system, calculating achievements by using 40 quantitative indicators. However, it should be noted that these indicators have little overlap with the measures used in the international university rankings. When NTNU is assessed by the government, it is to see if national goals regarding public goods and the policies aimed to shape its way of organizing its activities are implemented, not to stimulate efforts to improve its international ranking. 'Excellence' is a subordinate, not a superordinate goal, which is just hinted at in the documents. NTNU's national and international reputation is not an outspoken concern of the government.

The goals and the related parameters set by the government reflect a broad set of expectations regarding public goods. The policy documents leave no doubt that the Norwegian government is strongly involved with NTNU and does not shy away from intervening with the administration if goals are not met. The award letter, including the development plan with its assessment parameters is clearly a device to discipline NTNU to supply the expected goods. It is also a bureaucratizing device, which is evident from the prose of the document and its emphasis on goals and assessment parameters that invite NTNU to implement bureaucratic measures. It should be noticed that the documents from the government contain no reflections regarding public versus private goods. Implicitly, private goods, such as individuals getting an education or the industrial exploitation of university research, are seen as public goods since they contribute to the BNP and the national income.

An even stronger disciplining mechanism is the model that the government uses to calculate the yearly grant to the institutions. The model consists of two main parts, a fixed basis and a set of incentives. The incentives are calculated from selected assessment parameters that are indicators of last year's 'production': the number of credit points taken by the students, the number of graduates, a measure of international student exchange, the number of $\mathrm{PhDs}$ who have graduated, publications, and the grants from the EU, the Research Council of Norway, and other sources. NTNU uses a modified version of the model in its internal distribution of resources to discipline the faculties and the departments to pursue the politically decided incentives. The calculated budget objectivizes the pursuit of quantitative performance indicators and makes the distribution of resources more or less non-negotiable.

Thus, the calculated budget model together with the award letter imposes an impersonal command and control regime. This regime was accentuated when in 2004, the Office of the Auditor General sternly criticized Norwegian universities for lacking measurable goals. A statement of goal- and performance requirements in planning documents is the point of departure for goal 
and performance management of an enterprise. When formulation of objectives is missing, the consequences are that instruments and the responsibility for implementing plans are determined only to a small degree. At universities, there are no yearly planning documents at any level that state how results should be achieved, reported, and controlled. On this basis, questions may be raised if the universities satisfy pre determined requirements for governmental management of goals and performances with respect to the planning document structure. ${ }^{10}$

The universities in Norway, including NTNU, changed their ways, instigated by the Ministry's budget model and the policy goals set for the sector. Arguably, the resulting practice represents a morphing of the principle of 'management by objectives', which was made popular in the 1950s by management guru Peter Drucker (1954). The underlying idea of this style of management is that goals should be developed through participation of the employees. Furthermore, goals should be clear and limited in numbers. When NTNU's leadership faced the requirement to become auditable, they had to domesticate neoliberal ideals like excellence by articulating goals that allowed for assessment of the organization's achievements in terms assumed relevant to the government. To this end, the Board of the university over the years have approved strategy plans that contain a multitude of goals and ambitions. In this manner, at NTNU, neoliberal excellence has morphed into a multidimensional concern that spans from the recruitment of students to the quality of research. The latest strategy plan Strategy $2018-2025^{11}$ promises to pursue a total of 36 main goals. Ironically, this provides faculty with considerable room for navigation in their work. Nobody can effectively pursue 36 goals simultaneously. However, from interviews with heads of departments, we know that they experience the situation as difficult. How should they prioritize? For example, what criteria should be used when hiring new faculty (Lagesen, 2021)?

The award letters and the budget model are important technologies of governance of universities. According to domestication theory (Sørensen 2006), the use of such technologies requires sense-making and the development of a practice. What do the technologies of governance mean - how should they be interpreted - and how should they be enacted through practice? The strategy plan is an effort of sense-making with respect to Norwegian policies for higher education and research, but it contains only goals; no guidance for practice. Practice must be observed elsewhere. Of course, the strategy plan appears as a bureaucratic initiative to discipline the faculties and departments to enact the goals of the plan. This interpretation is supported by the fact that the faculties produce their own strategy plans that echo the NTNU plan, and then the departments are asked to produce their own version of that echo. On paper, this looks like a streamlined bureaucratic response to government policies, a set of interlinked technologies of governance that direct and discipline faculty, clearly imposing on academic freedom. It speaks to 
'the imagined university' that we referred to in Chapter 1, a strong vision of NTNU as governable.

To summarize, NTNU has made considerable efforts to conform to the dominant neoliberal perspectives and requirements. The domestication process has involved the development of governance practices that make the organization auditable by formulating goals and developing systems to measure and calculate achievements of these goals. These systems need to be co-morphed with appropriate symbolic interpretations to provide legitimacy, authority, and attractiveness to the new ways of timely accounting for what it means to be a university. Concepts like public goods, quality, relevance, and reputation are mobilized to this end. The main effort to comply with all the requirements from the government has involved bureaucratization.

By pursuing knowledge, we shall prepare for the future, creating new, green, and profitable jobs and a better and more efficient public sector. Therefore, good access to education is central. High quality of education and research is important to both a well-run work life and industry and to a continued stable development of the Norwegian welfare society. Norway shall have world-class research communities that participate in generating knowledge that may provide humanity with a better and richer life. ${ }^{12}$

Such grand statements require interpretation. We showed in the previous section how such expectations were turned into more outspoken aims and made even more concrete through the construction of comprehensive sets of assessment parameters. There is little doubt that NTNU's leadership has received the messages. This is evident from the way they have appropriated the policy makers' key words, repeatedly performing a discourse of ambitious goals to students, faculty, and, above all, in meetings with deans and heads of departments. However, the effect on everyday talk among faculty members and students has been modest. Rather, there is a faultline between the goal-speak of university leadership and the circulating narratives about bureaucracy and overwork. Faculty members are more concerned with struggles and perplexities regarding temporary employment, the burdens of teaching administration and reporting required to serve the metrics system, time squeeze, and the increased bureaucracy involved in research management. We explore this in greater detail in Chapters 3 and 4.

The budget and the budget indicators influence faculty and students at NTNU much more than the goal-speak. This influence is mediated through the setting of local goals of faculties and departments with respect to teaching and research. The goals mainly reflect the parameters present in the incentive system of the budget. The metrics create a sense of transparency, which allows for articulation of discontent with failure to reach the goals. This may make the everyday life of faculty more stressful, sometimes expressed by 
grievances about the workload and complaints about the frequent nudging about improved performances. The sub-politics (Beck 1997) voiced in the university corridors is concerned with resistance, guidance about quick-fixing, deconstruction of the goal-speak, distrust and indifference toward university leadership, and unfair distribution of resources. 'Excellence' is particularly interesting in the context of sub-politics since it is contested. We return to this in Chapter 3.

We have already indicated that the excellence concept has morphed at NTNU, but also at the Ministry of Education, which in recent years has been more reserved with using 'excellence' as a general goal for the Norwegian university sector. Rather, it has been replaced by 'high quality' as the key concept. This is found in both higher education and research policy. 'High quality' is defined in quite mundane terms. As we have seen, the need for easily available indicators has a moderating effect, which should be evident from the proposed parameters from the Ministry. The ensuing metric interpretation of quality seems removed from the international discourses on excellence. However, it reflects a political pragmatism that emphasizes economic effectiveness. There is nothing fancy about it. The university rankings are not mentioned in the award letters.

When we look at the rhetoric used by NTNU leadership related to university rankings, we observe a sobering process. In 2009, the Board of the university approved a strategy plan that aspiringly stated that 'Our vision is that NTNU in 2020 shall be recognized internationally as an excellent university'. This was made more specific in the aim that 'NTNU as a comprehensive university is among the top $1 \%$ in the world' ${ }^{13}$ When a new strategy plan was decided in 2017, ambitions changed. 'In 2025, we have achieved our most ambitious goal; all our departments have academic groups proven to be at a high international level in at least one of their core areas'. ${ }^{14}$ NTNU no longer aimed to be excellent, measured according to international rankings. Rather, some of its research groups should be excellent, but without any reference to how that could be determined.

The changed ambition coincided with the merger in 2016 with three regional colleges. The merger meant a large growth in the number of undergraduate students as well as the inclusion of three institutions with substantially weaker traditions of academic research. This fundamentally changed the ecology of the university. During the financial crisis of 2008 there were calls for merging some campuses of the University of California, as well as in other multicampus public university and college systems across the US. The practice has intensified during the pandemic. Similarly, over the last few decades university leaders have pushed for the consolidation of smaller departments. In both cases the goal has been to save money, while resistance is strong and merging organizational cultures has been difficult. There has been a growing scholarly literature on such mergers in academia globally and it is an important topic for future research. 
Arguably, after the merger at NTNU leadership redefined 'excellence' by leaving out reference to rankings and the university's international reputation. The concept was redomesticated with a greater emphasis on public goods (NTNU's relevance to the larger society), rather than its international standing. The emphasis was on improving the overall quality of activities, on becoming an even better social partner in efforts to develop industry and society in the whole of Norway, and on offering a set of more unified education programs. ${ }^{15}$ Thus, the meaning of being 'excellent' was morphed into 'socially relevant'. Research was not highlighted in the documents, besides expectations that NTNU should be able to gain more external funding for research and to strengthen its national role with respect to engineering education and research.

Unsurprisingly, when the world ranking of universities for 2020 was published, NTNU was ranked in the interval from 401-500, compared to the interval 251-300 in the 2017 ranking. This remarkable drop was given scant attention by university leadership until the pro-rector for research, Bjarne Foss, was challenged by the national university newspaper Khrono about the issue. Then he stated that

We are concerned with ranking as one of several measures of quality. The rankings are important and are actively used by those within academia .... We use the rankings as one of several inputs to develop professional quality and reputation. We follow and analyze why we are placed as we are on different rankings. We use them both internally and externally. ${ }^{16}$

How they would be used, has remained opaque, since there is no public discourse about rankings at NTNU. No new measures or initiatives to improve the ranking have been announced. NTNU faculty have voiced little concern regarding the changes. Lagesen (2021) observed that interviewed heads of departments said that their dean required that they should give priority to research excellence, measured by the number of publications in high-ranked journals. Others felt squeezed because they were expected also to emphasize experience from and competence in teaching. Improving the gender balance was an additional concern. Interviewees said this made their management of hiring processes challenging because they received little guidance from the dean or the administration about how to prioritize. Increasingly, the previously dominant emphasis on the research excellence of applicants has been replaced by an accent on 'suitability', an increased weight on teaching skills and academic service work.

NTNU overall has employed a 'soft' approach: excellence issues silently were morphed into 'high quality'. The economic incentives implemented in the budget model are based on quantitative production indicators, and they are mainly directed at departments and not at individuals. National evaluations of departments and research groups are undertaken every five to 
ten years and they have little consequence. However, as mentioned above, the metrics applied to measure performances provide a kind of transparency that sometimes is used by deans in exchanges about local distribution of resources.

Thus, NTNU's strategy for quality depends on governmentality: it is normal for academics to strive to improve their performances with respect to teaching, research, and other relevant areas. In this way, important aspects of academic freedom are respected formally, although quality governmentality definitively represents a moral pressure to work harder. To be fair, there have been some modest initiatives to encourage excellence, such as a program to foster research proficiency among a select, small group of promising young scholars. Also, economic resources, training, and advice are provided to support grant application writing, particularly to EU's Horizon programs and the European Research Council. Still, NTNU's quest for 'high quality' is, above all, a discursive undertaking intersected by pragmatism and a quest for relevance. Thus, excellence-speak is infrequent and moderate. This is reflected in NTNU's relative success in obtaining research grants where the criteria are a mix of 'excellence' and 'relevance', such as in programs that fund research centers focusing on sustainable energy and research-based innovation. It has considerably less success with respect to winning bids for centers of excellence and prestigious funding from the European Science Foundation. Occasionally, NTNU leadership voices concern about this, but they appear uninterested in finding explanations.

\section{UCLA - a Corporatizing University}

Like many public universities in the US, we showed in the previous chapter that UCLA is part of a system called the University of California (UC) that has ten campuses. The UC system shares many resources and regulations, but each campus has considerable autonomy in distributing resources and interpreting the shared regulations. Perhaps that autonomy is most obvious in their budgets. UCLA gets about 5 percent of its annual budget from the state of California through the UC system office. All the other UC campuses get increasingly larger shares of their budgets from the state government. UCLA gets more of its budget from private donors and extramural research funding than any other UC campus. The other UC campuses have less income from research and donors. Once accrued, UCLA decides how to allocate its own resources, as do most other US universities.

In sharp distinction to universities in most of the rest of the world, not the least in Norway as shown above, there is very little regulatory oversight of US universities beyond their campuses. That is, there is no national government agency regulating universities. Certainly, universities are subject to the legal requirements placed on any employer, as well as the legal requirements for managing the funds they receive from extramural sources, ranging from governments, corporations, and private donors. However, all decision making 
about teaching and research, admissions and hiring, as well as allocation of resources, is made within each university.

An important aspect of corporatizing is the changing evaluation practices. These changes may be described as a move from pondering quality to gaming metrics and governing with algorithms. This move is problematic since the judgment of quality in craftwork such as academic research and teaching is complex and impossible to teach a bystander quickly. A friend of Sharon who has been studying poetry since the 1960s and is now a panelist for a national poetry award, said that her judgment about the relative merits of poets' work is based on 'decades of close reading of poetry'. In any field the criteria for judging quality are learned from long apprenticeships in the craft of making and transmitting knowledge, along with learning the values associated with those knowledge practices.

In his Zen and the Art of Motorcycle Maintenance: An Inquiry into Values Robert Pirsig addressed the metaphysics of quality and concurred with Phaedrus that 'good' is a noun, not an adjective. Following the Challenger Space Shuttle explosion, the Rogers Commission tried to locate the cause. Roger Boisjoly, an engineer, testified about a problem with one part of the assembly: 'I cannot quantify it, but I know that it is away from the direction of goodness.' That did not convince the managers who had overruled the engineers. Later the physicist Richard Feynman commented on Boisjoy's testimony by remarking on the differences between engineering and managerial ways of knowing. We have learned from a body of research conducted during the 1980s in Science and Technology Studies (STS) that the design of 'expert systems' in artificial intelligence included a fundamental flaw, linked to the old distinction between 'knowing how' and 'knowing that'. The craft knowledge of expertise is about knowing how to craft something and make sense of it; expert systems focused on knowing information (Collins and Kusch, 1998; Suchman, 2008). From metric evaluations systems we can know that certain numbers of papers were published, but we cannot know how their colleagues decided which of the published papers were of higher quality. In Boisjoly's terms, we do not know which papers were 'in the direction of goodness'.

A fundamental task in teaching and research, as well as administration is evaluating quality and then using that judgment to allocate scarce resources, including our own attention. From 1980 to 2020 the evaluation of quality in academia first shifted to defining a set of 'metrics' and then using them to build algorithmic or model-based governance for universities as a whole. Another example we will address later is the shift from university-generated software for admissions, budgeting, financial management, personnel matters to products first made for corporations during the 1980s and 1990s, then repurposed for universities when it became outmoded in the business world. Many academics have colluded in these processes, justifying these choices in the name of efficiency and transparency. To turn judgment criteria (knowing how) into 'metrics' (knowing that) and then use those metrics to build 
algorithmic governance does not make the evaluation of quality transparent and fair. It does something else. That 'something else' is the topic at hand.

How do administrators and faculty members at UCLA decide how to allocate scarce resources to new lines of inquiry, new infrastructure, faculty hiring and promotion, and graduate student admissions? In part that is done by evaluating what has been accomplished. As mentioned earlier, for centuries universities, scholars, their research, and their teaching have been evaluated. Around the world different practices have evolved for evaluating the current state of universities, along with their centers, programs, and departments, as well as the work of individuals, plus how to use those evaluations for longrange planning. In some countries there has been a relatively stable prestige ranking of universities for a century or more. In those places many students, academics, policy makers, funders, and citizens would have assumed that the best scholars were at university $\mathrm{A}$, the second best at university $\mathrm{B}$, and so on, and allocated their resources accordingly. In turn, the consistent accumulation of resources at certain places meant that their continuing prestige was assured.

Since the 1980s, UCLA in its quest for excellence has, like many other universities, been measuring itself among others and measuring those within the campus according to a specific set of metrics: citation counts, impact factors, and rankings. In turn, it has chosen to allocate resources within the university from graduate student admissions to faculty promotions, by adopting a set of algorithms adapted from corporate governance models developed in the 1980s and 1990s. Meanwhile, as we saw above, NTNU has followed another path. UCLA's focus on metrics has coincided with a widespread awareness that the crucial measures can themselves be 'gamed', meaning that each of the measurement criteria can be deduced and then those variables can be maximized, strategically. The entire process occurs at the same time nation states have increasingly focused their economic policies on productivity, first based on labor and then on capital. Recently newer forms of productivity are being measured, including the 'social good', as we see at NTNU.

A notable change in evaluation strategies emerged in the 1980s that, in turn, led to massive changes in the management of universities in some places. The Thatcher government in the UK argued that university budgets were wasteful, and after severe funding cuts in 1981, they began to correlate future funding with a 'research assessment exercise' (RAE), launched in 1986. That process has been modified over the years; since 2014 it has been called the Research Excellence Framework (REF). The UK system of evaluating researchers, research programs, and universities eventually led to a set of public rankings that massively challenged the traditional prestige hierarchies of universities. Many date the launch of so-called neoliberal university infrastructures internationally with that 1986 UK assessment scheme, focused on 'quality' defined by certain quantitative measures that had become possible with new computing resources for tracking attention to research publications. Scientometrics 
is the scholarly field that has developed a complex set of tools for assessing those data to understand some of the ways in which knowledge is being made. The RAE and REF made use of some of the findings of that field. Later in their widely circulated 2015 Leiden Manifesto the scientometricians strongly criticized the faulty use of their work in research assessment schemes (Hicks et al., 2015).

By the late 1980s, colleagues were deciding to cite each other's articles. Undergraduates at Rice University were casually using the phrase 'salami science' to describe research projects that were designed to generate the largest possible number of papers, the 'least publishable unit' (LPU), that will go into so-called 'high impact' journals. By the 1990s the consumption of articles had become another important metric; many already had learned how to 'download' certain papers repetitively, but in various patterns, not easily monitored. Students had learned to enter fields in which they could generate early in their careers the greatest number of papers. Some were quite cynical about the process, but others clearly believe that quantity must mean quality.

Just as the metric evaluation system was becoming widespread globally in academia, there also were strong criticisms of its short- and long-term implications for the quality of research and teaching, as well as for university infrastructures and conditions of employment. The criticisms have intensified, such as studies of the then new 'audit cultures' (Strathern, 2000) and coalesced into the field of critical university studies as well as many commentaries on 'the neoliberal university' (see Chapter 1). The redefinition of quality through quantitative measures has led to the worldwide restructuring of universities, research, teaching, and careers in academia. That transformation has been described and analyzed extensively and we will not review that history here but take it as the consensus position. Our question is how that 1980s revision of evaluation practices and the remaking of prestige among universities shapes current practices at UCLA.

While there has been a worldwide shift toward defining the quality of scholarship and teaching by quantifying certain forms of productivity, there remain many different practices for evaluating the current state of universities, as well as how to use those evaluations for long-range planning. In some countries those evaluation reports are linked to national budget planning, but not in the US where there is no centralized oversight or funding of education at the national level and little such oversight at the state level. Despite all that local variation, attention to global indicators has become important for many policy makers internationally, as well as at specific universities. However, we note a stark contrast between the significance of productivity metrics and rankings for decision making at NTNU and UCLA. At NTNU and in Norway they attract little attention, while extramural rankings are followed quite carefully at UCLA and quantitative intramural evaluations are extensive and continuous. This heightened concern with quantitative rankings at UCLA correlates with its extensive corporatizing practices. 
Since 1982 there has been a meticulous ranking of US university doctorate programs generated approximately every 12 years by the US National Research Council (NRC). ${ }^{17}$ Its focus is on ranking departments among US universities by field. It once was very influential within the US, but its prestige was challenged ten years ago. Now few academics within and beyond the US are aware of the older NRC rankings, increasing the significance of the annual international rankings. After 1995 the NRC ranking methods were reassessed, due in part to all the new international university ranking systems. That led to a series of postponements for its subsequent controversial report, expected in 2007, but not issued until 2011. The new methods triggered many debates about how to measure the relative prestige of the programs, especially the shift from accumulated to current reputations, as well as the decision to announce clusters of universities at the same general rank, rather than listing them separately, in rank relative to each other. The NRC rankings are supplemented by surveys of graduate students; the influential, private Alfred P. Sloan Foundation sponsors the US National Association of Graduate-Professional Students (NAGPS) and provides the funding for a National Doctoral Program Survey (NAGPS) assessing graduate programs using nine criteria. ${ }^{18}$ Succinctly, the 2011 NRC report and the NAGPS survey showed that many of the older prestigious programs had been losing status and the newer ones were gaining rapidly when different evaluation methods were used.

US universities and colleges also are evaluated regularly by regional and professional accreditation agencies to establish that they are meeting basic standards, a process that began in 1952. In addition, research universities usually require their own academic centers, departments, and programs to conduct evaluations of their teaching, research, and planning every 5-8 years. These evaluations typically take one year and might involve many kinds of reports, including future faculty hiring priorities, curriculum planning, and surveys of their faculty, students, alumni, and so on, on many topics. For example, the UCLA Academic Senate also appoints a committee of leading faculty members from other departments to evaluate each department. In addition, there are evaluation reports by leaders in the same field at other leading universities around the United States, based upon their own visits, investigations, and interpretation of reports generated by the department. (The members of that committee are appointed by the relevant university dean.) The department uses these 5-8-year evaluations for assessment/ planning, as does the dean and the university.

Some departments post these evaluation reports on their websites, but others do not. Typically, a department will spend one year preparing for its evaluation, while strategically emphasizing its strengths and establishing its readiness for new initiatives, if only given adequate resources to do so, such as new faculty positions, more support for graduate students, and so on. The department will invoke any rankings it might have received from international 
and national groups, public and private, as well as any national or international recognition any members of the department might have received. Sharon has observed how new faculty positions can be allotted as a result of such reviews.

In the US there are also commercial rankings of colleges and universities, including graduate and professional programs. The primary target audience for those commercial ventures are prospective students and their families who might be uninformed about the rankings listed above. However, some of the commercial rankings increasingly influence public opinion more generally, as well as policymaking, even decision making within university administrations. The best known is a cluster or guides from the US News and World Report launched in 1983, such as America's Best Graduate Schools. ${ }^{19}$ US universities submit extensive information called the Common Data Set (CDS) to the various public and private university rankings groups. To do so universities now often have special offices devoted to the task of assembling that information, which they also use for their own institutional planning and decision making. At UCLA much of those data are aggregated by the Office of Analysis and Information Management (AIM). Another source is the UCLA Department Data on Graduate Admissions \& Enrollments. Considerable data on doctoral programs by department are available at many university websites, but clearly missing on others. At UCLA the data on number and rates of admissions, enrollments, degree completion, and normative time-todegree are aggregated into five-year averages, along with data on graduate students' gender, ethnicity, and nationality. These data, taken together, are regarded as measures of success for a department's graduate degree program, and the data are used to compare departments across fields in the university, and within a field across many universities. ${ }^{20}$

The variables in those data are the topics of current concern in the US. For example, in the US students apply to many graduate schools simultaneously, so most departments at the same rank are admitting the same students, hoping they will enroll in their program, rather than the others. Hence, a strong ratio of students who in fact enroll among all those admitted is about 50 percent. (This ratio is called the 'yield', a term taken from agriculture.) There is considerable variation in the yield rate across departments at UCLA and that ratio is used to compare quite different kinds of departments.

During US participation in World War II and the Cold War (roughly 19421990) the US government wanted more scientists and engineers to contribute to 'national defense' and pushed universities to generate more of them. For example, historian David Kaiser (2002) found that in the UC Berkeley physics department the pressure to generate more advanced degree recipients more quickly required a reframing of the degree requirements, including redefining 'do-able' dissertation projects to those that could be completed in less time. Faculty who did not cooperate with the reduced time-to-degree were not promoted. 
The UCLA Department Data on Graduate Admissions \& Enrollments includes demographic data on the students. The categories enumerated in some university datasets are African American, American Indian, Alaska Native, Asian, Hispanic, Pacific Islander, and White; students and employees can choose more than one classification. Note that the labels Asian and Hispanic can include both Asians and people with Hispanic surnames from anywhere in the world, as well as Asian Americans and Hispanic Americans. Hence, a department with many students and faculty members from Asia, Latin America, and the Iberian Peninsula might have many 'minorities' but few 'under-represented' groups from the US. Similarly, the category 'White' can include people from anywhere in the world. The term 'minorities' in some university data includes only 'under-represented' minorities, meaning those groups that are underrepresented in academia, relative to their proportion of the general US population. For example, Asian Americans are not included in some of the minorities listings because they are not considered to be under-represented in academia, relative to their proportion in the general US population. These vague ethnicity, race, and nationality listings vary significantly by departments at UCLA. Some might have a remarkably high proportion of foreign students from countries that provide funding for study abroad in those fields. Many departments admit few foreign students who cannot provide their own funding because such students will require substantial funding from the department.

There are over 5000 colleges and universities in the US, and there are more than 275 in California. In 1970 the Carnegie Commission on Higher Education developed a complex classification system of post-secondary institutions of higher learning, regularly updated. ${ }^{21}$ (It is now maintained by the Center for Postsecondary Research at Indiana University.) Those categories have become quite influential. Four of the seven primary classifications focus on the most advanced degree awarded: doctoral degrees, master's degrees, baccalaureate degrees, and associate degrees. Of the Doctorate-granting universities, 130 are labeled R1, designated as having 'very high research activity', and the 132 R2 universities have only 'high research activity'. Furthermore, within those $130 \mathrm{R} 1$ universities each will have designated a special group as their peer or comparable institutions. UCLA's 'comparison 8' includes four private schools (Stanford, Harvard, Yale, MIT) and four public universities (University of Michigan, University of Virginia, SUNY Buffalo, and the University of Illinois at Champagne Urbana). ${ }^{22}$

Each university's short- and long-term strategic planning in everything from student admissions criteria to faculty salaries will be considered primarily within that frame, while working to both maintain and its position among its comparable institutions and to advance its status to a more prestigious group of peers. One way to do that is to increase its ranking among the best-known ranking systems. 'There's no rankings problem that money can't solve', says Michael Bastedo, director of the Center for the Study of Higher and Postsecondary Education. ${ }^{23}$ 
Earlier examples include Stanford University and the University of Southern California. Until the late 20th century each was considered within the state to be a school for those who did not meet the entrance requirements for the University of California. Wallace Sterling who

served as president of Stanford from 1949 to 1968, a period that produced a 40 percent increase in students, mostly at the graduate level, and a 170 percent increase in faculty members. He was credited with playing a crucial role in the development of Stanford's national and international reputation..$^{24}$

He raised $\$ 400$ million (worth about $\$ 2.8$ billion in 2021) from donors which he first used to recruit nationally prominent faculty members in STEM fields and engage in massive reconstruction of the campus and its reputation. James H. Zumberge, president of the University of Southern California, 1981-1990, followed the Sterling template; he raised $\$ 642$ million (worth $\$ 1.312$ billion in 2021) to launch an impressive faculty hiring and campus construction program. ${ }^{25}$ Similarly, in the 1960s UCLA was still considered the 'southern branch' of UC Berkeley with UCB having far more status. In the QS Higher Education Rankings of 2004 UC Berkeley was ranked \#2 in the US; UCLA was \#15. In 2021 UCB is \#4 and UCLA is \#5. ${ }^{26}$ During 2014-2020 UCLA raised $\$ 5.5$ billion from donors.

Clearly the rank of universities is determined quantitatively by generating data on certain variables and that rank can be shaped by maximizing certain variables. Many universities have turned to using those data to make decisions about the allocation of resources within the university. UCLA is among these. The model implemented in 2021 is explained in the following way:

The Bruin Budget Model is a hybrid of commonly used budget models and is informed by the best practices of peer organizations. The goal is to provide transparent incentives for units to pursue revenue growth opportunities, to find efficiencies, and to exhibit strong expense management. ${ }^{27}$

Compared to the budget model used by the Norwegian government and the internal model adapted by NTNU the general reasoning is similar. The models combine the use of a relatively stable historical component and a variable component based on performance indicators. In this way, the models are supposed to provide incentives for faculty to do 'the right things'. When we look at the models in more detail, they differ in ways that partly reflect UCLA's greater dependence on donations and fees, but also differences in the metrics that the universities produce. A major difference between NTNU and UCLA is that NTNU's model is constructed based on political concerns of the government, while UCLA's model is created from rankings and comparisons with other US universities. 
In addition to the $\$ 5.5$ billion UCLA raised in donations in 2014-2020, it also has received over $\$ 1$ billion in research funding each year for over ten years which includes large fees for 'facilities and administration' services provided by the university. The state pays about 5 percent of the budget. What is being counted is what matters to the university. What is being excluded matters to many of the students, staff, and faculty. Unlike Knut, who has observed little discussion about the budget model at NTNU other than among deans concerned about 'technical' issues, primarily the weighting of different study programs, Sharon has encountered students, faculty, and staff coming to her and sharing stories and asking questions:

- Why and how do many strive to become what the platforms want us to be, learning to score highly in the evaluation games surrounding us?

- How do management platforms undermine equity, inclusion, and diversity?

- Why are so few interested in the epistemic assumptions, exclusions, and goals built into those platforms?

- Who designs, sells, and buys them? Why?

- What are the strategies for resisting this neoliberal algorithmic governance?

One response to such questions is to look at the software platforms that now structure five crucial, everyday campus practices: student admission and monitoring, faculty hiring and evaluation, research management, teaching logistics, and financial coordination. Typically, those five activities are built on 'customer relationship management' (CRM) platforms, first developed in the 1970s and in wide US use since the 1990s. ${ }^{28}$ Increasingly, CRM software has been conflated into the newer 'human capital management' (HCM) platforms, as has happened at the University of California system, through a contract with Oracle Corporation for their product PeopleSoft. ${ }^{29} \mathrm{CRM}$ is also the basis for two other platforms used at UCLA, as well as many other US universities: SLATE, an admissions system, and Blackbaud, a product for managing funding campaigns and donor relations.

The CRM platforms often have been seen as rather prosaic devices that turn routine university-based work into easy, but tedious Fordist tasks, done quickly and simply while easily monitored and made more efficient. However, generations of research on Fordist practices have shown that they redefine workers, intensify regulation, and enhance regimes of accumulation. The CRM platforms now regulating campuses are redefining us campus workers, the students, faculty, and staff; we have experienced new subject formation, encouraging us to do the work needed to operate the CRM platforms effectively, rewarding us for providing the data the platforms want. The university becomes the place that provides the work that makes for good rankings, which raises questions like:

- What happens when the system does not work?

- What happens when we workers do not provide the data the platforms want? 
- What happens when we do not use the software as we should?

- When we do not shift our work to enhance the data the software wants, what are the implications and alternatives, if we do not use these masters' tools to teach and learn?

- What happens if the students, faculty, and staff become Luddites, ignoring or hacking the platforms that govern us as knowledge workers?

In the early 2000s many universities began to plan moving their administrative computing from mainframes to server- and cloud-based technologies. At that time many also evaluated whether to move away from universitydesigned software which had been maintained and repaired on campus to outsourced software designed and maintained by vendors. Like most universities UCLA decided to outsource, as it did many other services. Many STS studies of technologies including computing have shown that local design, maintenance, and repair can lead to resilient, flexible tools, responsive to local cultural practices. Many oral histories of university 'mainframe maintainers' report on the complex relations between the computing staff and the university users, developing resources for local practices (Meyerson and McClenon, 2017). The socio-technical networks of the vendor supplied platforms now used in many UK and US universities have reshaped local practices significantly (Williamson, 2018). The decision to outsource many services was made in the name of efficiency and cost-savings; studies of shown that has not been the result (McClure, 2014, 2016, 2017).

During a break in an undergraduate seminar on research methods Sharon was teaching a few years ago, she overheard a student saying, 'I hate this place!' Others agreed. As the class resumed, Sharon asked what they had meant. They said they felt manipulated by administrative platforms that praised them while erasing needed resources. As UCLA has sought improved rankings the student applicant pool has increased enormously: more apply to UCLA than to any other university in the US. In turn UCLA has become very selective in admissions. Very interestingly, as admission has become more difficult, the family incomes of the admitted students are much lower than in the past. To recruit the admitted students UCLA promises much to them in resources, just as physicists and astronomers engage in 'promissory discourses' to gain access to scare resource for their facilities.

However, those high-achieving, low-income students arrive on campus to find much less than they need and were promised. Yes, UCLA is a very privileged site, but that wealth is not distributed equitably, and there are huge resource disparities. One colleague long ago said it was better to live in a rich place where even the weeds get watered. Even so, during droughts some parts of campus get more water than others. Another example: when walking into the offices of the UCLA Gender Studies Department where the administrative staff members work, they tell stories of terrible stress; the staff members worry much over the actions required by the new HCM platform 
being implemented across all ten campuses of the University of California system. This past year the financial platforms systemically thwarted the pay of student workers for several weeks. During the pandemic UCLA 'laid off' many student workers without providing them with information on how to access government resources for the unemployed.

Those outside the US higher education ecology might not know that most US students are receiving financial assistance in two forms: working up to 20 hours a week on campus and taking on debt to be repaid in future decades, at interest. In turn the students must use some of their salary to pay their student fees. Research shows that their remaining wages are not enough to cover their rent in student housing. Those who manage the payment of those student workers worry about the students. The platforms are designed to find the misuse of funds. Of course, many of us would say the platforms are working fine. They indeed are detecting misuse, but it is not by the workers or their managers; it is in the allocation of resources. Meanwhile, many enjoy gaming the new algorithms. As described earlier, many conduct what is called 'salami science' and learn to generate the greatest number of papers in the shortest period of time. Many are socialized to believe that quantity must mean quality. US universities now know how to 'game the rankings' as suggested earlier. Articles abound on how certain schools have had their international rankings increase stunningly in short periods of time. That increases their revenue, enabling them to attract better students and faculty members, as well as more private donors, which in turn increases their rankings. That is a very destructive 'positive' feedback loop. Maximizing the metrics has led to more extensive use of the 'customer satisfaction management' platforms.

US universities, including UCLA, have increasingly worked to maximize five so-called 'revenue streams' of student fees, student campus residence fees, facility fees for sports and cultural events, extramural research funds, and private donors. CRM is designed to generate and maximize those revenue streams. Of course, CRM sees everyone as a customer or a provider of services to customers; students are customers and faculty provide them with services. The customers expect a degree in exchange for the transaction of paying tuition and attending classes. However, what happens when the student customers are no longer satisfied and there is no place to post their discontents in the CRM audits? The software does not understand those problems.

What happened when Covid-19 closed the campus from March 2020 to August 2021? Many students have not wanted to pay full tuition at expensive universities for online courses and many did not want to return to campus to live in campus dormitories while taking online courses. Many have not wanted to return to campuses during a pandemic. University administrators seem to believe they are confronted with financial collapse if they cannot get students to pay fees and return to campus. In order to maintain their 'revenue streams' (their income) they began advertising their pandemic safety plans. As one professor at CUNY wrote in the New Yorker, our universities are facing something 
worse than financial bankruptcy: it is moral bankruptcy (Robin, 2020). This perfect storm has been brewing in universities for decades. The software for 'customer relationship management' and 'human capital management' as well as the related risk management software had long ignored what they could not incorporate into their algorithms. It is a problem of categorical thinking, ignoring what is excluded and reifying what does fit into the classification system. It is a problem of misplaced concreteness, a clear sign of ideology. What has been left out of the software, the algorithmic governance? It is the university as a site of knowledge making, the epistemic work of teaching and research, the meshwork at faultlines. CRM and HCM have excised all that as outside their categories and their metrics. CRM is designed to generate revenue streams. The detritus of CRM is the university: our commitment, determination, curiosity, and passion for learning and making knowledge.

\section{Conclusion: The Co-morphing of Metrics and 'Excellence'}

When we began to discuss this book, we believed that primarily we would inquire into the dynamics of neoliberal universities by analyzing the quest for excellence at NTNU and UCLA. Our understanding then of universities' definitions of 'excellence' was linked to international university rankings. Our assumption was that their quest for excellence involved a singular focus on these rankings and how to improve our universities' position. This was what it looked like at that time. We learned during the course of our inquiry that our initial assumption had to be modified. As we noted in Chapter 1, the concept of excellence is fundamentally vague. The circular definition found in Merriam-Webster emphasizes the point: 'Excellence is the quality of being excellent'. ${ }^{30}$ The meaning is contingent on context. As we have seen, the meaning of the concept with respect to university practices is mediated by the local political economy and academic culture. Financially, UCLA depends on its academic reputation as measured by its position on national and international rankings. This makes these rankings vitally important; a concern that permeates the way that UCLA distributes resources and conducts assessments. It also shapes the metrics constructed by the university. The ensuing corporatizing practices flow from the co-production of 'excellence' and performance metrics. No excellence without indicators.

With NTNU, the story is different. The ranking held some significance to the university, but not to the government that funds NTNU. Rather, after some political interest in the rankings ten to 15 years ago, the government has preferred to emphasize different kinds of performance indicators. Reputation is important to the university, but it is determined by popular assessments and student surveys, not on international ranking. Situated in Trondheim, the administration at NTNU assumes that the city's standing as a nice town for students to live in is at least as valuable as any recognized academic 
achievements. Moreover, NTNU's income is not directly affected by its reputation, but decided from a heterogeneous, pragmatic, and mundane set of indicators with little overlap with those used in the international rankings. The government's focus is the production of public goods, assessed by political priorities. Thus, in recent years, NTNU leadership has replaced the concept of excellence by 'quality', which often is linked to 'relevance'.

This does not mean that NTNU is excused from the discursive pressure of 'excellence' as an international ambition for universities, but it is quite effectively moderated through the government's presence in university politics and decision making. This presence accentuates public goods and social relevance and clearly circumscribes the autonomy of NTNU, even if the budget system in theory provides considerable financial autonomy with regard to how the university spends its resources. The state of California is more absent with respect to UCLA. This does not necessarily provide UCLA with greater autonomy than NTNU; we are observing different forms of autonomy. We expand on this in the next chapters.

Thus, the juxtaposition of the political economy of the two universities shows distinct differences, to the extent that the shared use of the label 'neoliberal' for both NTNU and UCLA appears to offer limited analytic benefits. On the one hand, NTNU and UCLA share a reliance on metrics as tools of governance, metrics that largely measure performance and invite internal competition for resources through productivity. On the other hand, the indicator system constructed by the Norwegian government includes many other achievements than those usually applied to measure excellence at UCLA, leading NTNU to follow different excellence practices than UCLA. In this way we claim that metrics are co-morphed with 'excellence'; neither of the two concepts have a stable meaning, but on the other hand, they cannot be made sense of without reference to each other.

In the coming chapters, we analyze some further consequences of these different political economies and their enactments. First, in Chapter 3, we explore how academic freedom intervenes and the ensuing processes of collegial relations and the making of academic work cultures. This includes a discussion of different assessment practices and the role of factions in decision making at the level of departments. We inquire into how the effects of the comprehensive bureaucratizing and corporatizing are mediated by academic cultures, thus focusing on what it means to live and work at universities that have domesticated neoliberalism differently. How have we experienced NTNU and UCLA?

\section{Notes}

1 For a succinct survey of McNamara's advocacy of systems analysis in corporate, government, and NGO leadership, see https://en.wikipedia.org/wiki/Robert_ McNamara. See too The Fog of War: Eleven Lessons from the Life of Robert 
S. McNamara a documentary film interview with McNamara in which he criticized his earlier work.

2 https://ucla.app.box.com/s/xj7xumhd40nh1scglvzw1pm9562kq8dy (downloaded October 13, 2019).

3 www.ntnu.no/documents/1277297667/1278198595/20180228_NTNU_strategi_ web_ENG.pdf/cb19eb5b-bd82-453d-ac54-8e89c67509e3, p. 13. (downloaded October 13, 2019).

4 www.youtube.com/watch?v=hnzCuhyuf4c (accessed June 7, 2021).

5 https://newsroom.ucla.edu/releases/knowledge-solves-brand-campaign (accessed June 7, 2021).

6 See https://brand.ucla.edu/ and https://shop.uclastore.com/c-312-apparel-accessories.aspx

7 Information about THE; www.timeshighereducation.co.uk/world-university-rankings/ and https://en.wikipedia.org/wiki/Times_Higher_Education_World_University_R ankings. About QS www.topuniversities.com/ and https://en.wikipedia.org/wiki/QS_ World_University_Rankings. About ARWU www.shanghairanking.com/ and https:// en.wikipedia.org/wiki/Academic_Ranking_of_World_Universities

UCLA typically has its own rankings site www.ucla.edu/about/rankings

8 www.uio.no/om/aktuelt/rektorbloggen/2021/ang.-udferdigelse-af-en-lov-om-detkongelige-frede.html (accessed May 5, 2021).

9 https://innsida.ntnu.no/c/wiki/get_page_attachment?p_1_id=22780\&nodeId= $24647 \&$ title $=$ Strategi + og + styrende + dokumenter $\&$ fileName $=$ Tildelingsbrev $\% 20$ fra $\% 20 \mathrm{KD} \% 202020$.pdf (accessed October 20, 2020).

10 Riksrevisjonen (Office of the Auditor General). Dokument nr. 3:3 (2004-2005), p. 2. Translated from Norwegian by the authors.

11 www.ntnu.no/documents/1277297667/1278300731/20180209_NTNU_strategi_ print_enklesiderENG3.pdf/b9f001ed-7236-4628-8874-27a78489c441 (accessed October 20, 2020).

12 www.regjeringen.no/contentassets/72751a723c654b029d7c04f67e171275/ tildelingsbrev-2021-for-norges-teknisk-naturvitenskapelige-universitet-ntnu-.pdf (accessed July 5, 2021, translated by the authors).

13 www.ntnu.no/styret/saker_prot/12.09.08web/60.08.pdf

14 www.ntnu.edu/strategy-2018-2025, p. 15.

15 www.ntnu.no/documents/1262755726/1262827395/VEDTATT_Fusjonsplattform_ 190815.pdf/bf5c2955-bf6c-4388-8152-8a18583e7210 (accessed February 9, 2021).

$16 \mathrm{https} / /$ khrono.no/nmbu-ntnu-rangering/ride-ride-ranking-pa-harvards-fang/ 401715

17 www.nationalacademies.org/our-work/an-assessment-of-research-doctorateprograms. The 1995 rankings appear in Research-Doctorate Programs in the United States: Continuity and Change. They include 3,634 programs in 41 fields at 274 institutions.

http://books.nap.edu/catalog/4915.html

The NRC 1995 rankings for science and engineering are also online at www.phds.org/ and www.phds.org/rankings/

$18 \mathrm{http}: / /$ survey.nagps.org//admin/manage.php

19 http://grad-schools.usnews.rankingsandreviews.com/best-graduate-schools 
20 UCLA graduate program reports: https:/grad.ucla.edu/programs/program-statistics/

21 https://carnegieclassifications.iu.edu/ and https://en.wikipedia.org/wiki/Carnegie_ Classification_of_Institutions_of_Higher_Education

22 https://apb.ucla.edu/faq/comparison-8-schools. UC Berkeley has a similar group of universities they use for benchmarking https://opa.berkeley.edu/sites/default/files/ucb_ standard_peer_group.pdf. The benchmarking practice is common in the US, see www. chronicle.com/article/in-selecting-peers-for-comparisons-sake-colleges-look-upward/

23 www.bostonmagazine.com/news/2014/08/26/how-northeastern-gamedthe-college-rankings/

24 www.nytimes.com/1985/07/03/us/wallace-sterling-led-stanford-u.html

25 https://about.usc.edu/presidents/james-h-zumberge/

26 https://dailybruin.com/2021/05/07/ucla-ranked-no-5-nationally-in-qs-worlduniversity-rankings

27 https://apb.ucla.edu/bruin-budget-model

28 There are 5 types of CRM. For a brief description and history of the genre since the 1970s see https://en.wikipedia.org/wiki/Customer-relationship_management

29 See https://fomucpath.ucr.edu/faq.html and www.oracle.com/applications/humancapital-management/what-is-hcm.html

30 www.merriam-webster.com/dictionary/excellence 


\title{
In the Shadows of Excellence and Neoliberal Interventions
}

\author{
Enactments of Academic Autonomy \\ and Strained Collegiality
}

\section{Introduction}

During late winter 2021, a brief exchange appeared in NTNU's newspaper related to the election of new representatives to the Board of the university. The candidates presented statements and programs. A predominant view was that the governance of NTNU was increasingly dominated by the top leadership, including the central administration. When asked to explain, the 'top-down' argument was extended to include the Ministry of Research and Education and its directorates that are concerned with higher education. The complaints centered on two issues: the outspoken efforts to govern university research and the lack of participation of employees and students in decision making.

We may recognize such complaints from the critical university studies literature that we reviewed in Chapter 1. It highlights the increased corporatizing of universities through the introduction of 'academic capitalism'. We also showed in the previous chapter how NTNU was intersected by a deluge of policy reforms, including the use of performance metrics and budget incentives, that provided for external governance. The situation of UCLA was different in this respect; not so much because external pressures were less important, but because of less direct interference from the state. However, in both cases it is pertinent to ask about the governability of the universities. To what extent may the daily tasks of faculty and student be governed 'top-down'?

In the previous chapters, we have outlined the political-economic context of NTNU and UCLA, observing the effects of different governmental engagements. We also explored the development of metrics for auditing academic activities. NTNU mainly adopted metrics and indicators used by the Ministry of Research and Education, while UCLA adopted auditing metrics from three sources. The three sources are instructive for both the governance practices embedded in them and the different relationships they facilitated within and beyond UCLA. Gradually UCLA has moved away from a set of metric management systems that were generated, maintained, and modified 
by people in universities and shared among them as open-source systems. The last ten years UCLA has shifted toward a set of metric management systems generated, maintained, and modified by private industry during the 1980s and 1990s when hierarchical, top-down, data-driven management practices were still widespread in the corporate world, even if they had not been recommended for some while. That is, the products in use at UCLA are corporate products from the last century with multi-layered management hierarchies embedded in them.

The first set of metrics practices had emerged in the context of universities providing data to the various ranking systems within and beyond the US, leading to the development of what is called the 'common data set' (CDS), an initiative that established common definitions for all the elements to be used in the rankings, which each university provides to the rankings groups. In turn, the UCLA CDS can link us to a wide array of numerical data UCLA collects about itself; the compilers are the UCLA Office of Academic Planning and Budget, the UCLA Office of Research and Creative Activities, and the Chancellor's Office of Data Analytics. The data from those offices often are circulated elsewhere as part of many monitoring schemes that exist to show that the university is in compliance with various state and national requirements for employers, such as safety, diversity, harassment, financial management, and so on. ${ }^{1}$

The second source initially was an informal coalition of people at various universities trying to design what are now called 'learning management systems'. Sharon worked at Stanford on one of the early versions of 'computer assisted instruction' (CAI) systems. By the 1980s there were many rather straightforward systems providing for quizzes and class record keeping. 'The first fully-featured Learning Management System (LMS) was called EKKO, developed and released by Norway's NKI Distance Education Network in $1991^{\prime} .^{2}$ For many years there were both local practices and much sharing in developing platforms for online 'collaborative learning environments'. ${ }^{3}$ In 2007 UCLA selected an early version of Moodle, a free and open-source LMS:

We looked at this decision as being a lot more than about selecting a technology - it was about a new direction for UCLA. First, it was a commitment to becoming part of a larger community of educators and institutions; second, it was about open source; third, it was about a common toolbox to support teaching, learning, AND collaboration; and fourth, it was about UCLA units and individuals working together to provide a common service that supports rapid innovation. Our goal is to benefit through contributing to and learning from a global partnership that holds values of access and cooperation matching those of UCLA. ${ }^{4}$

In 2008 the first platform for 'massive open online courses' (MOOCs) appeared. Sharon was involved in a 2010 Fembot Collective effort to design 
a MOOC platform that did not embed hierarchical pedagogies in its infrastructure. ${ }^{5}$ By the 2010s many universities around the world were using one of a small set of open-source and commercial products: Blackboard, Canvas, and Moodle. During the pandemic the multiple features of such systems were much more widely used. During summer 2021 UCLA shifted from Moodle, which it has used since 2007, to Canvas, developed by Instructure, a corporation owned by a private equity company. ${ }^{6}$ The third source of management metrics software systems at UCLA is a set of platforms designed in the 1980s and 1990s for corporate use. UCLA has bought such systems to use in undergraduate and graduate admissions (based on customer management systems), personnel evaluations (including for faculty members), budgeting, and fiscal management, as discussed in Chapter 2. These are the systems that are structured hierarchically and implemented alongside a 'hub and spoke' management structure, also from the last century.

One consequence of this series of algorithmic choices was that while UCLA has pursued excellence much more explicitly than NTNU, it has done so with strategies that follow specific corporate models from the last century with a focus on market share. Those strategies are pursued through top-down, hierarchical management structures by administrators, many of whom have never been academics. Meanwhile at NTNU, excellence was morphed into 'quality' and the interventions from the government have led to increased bureaucratizing, but again with top-down management by bureaucrats, many of whom have never been academics.

Many are unaware that there has been a vast and very expensive increase in the employment of administrators at universities. For example, at the ten campuses of the University of California,

management positions grew 13.6 times $(1258 \%) \ldots$ between 1975 and 2015. In the same period, UC students grew 2 times (100\%). Tenure track faculty grew 1.6 times (55\%) ... In the last five decades, UC management and non-academic occupations have grown substantially while tenured faculty have lagged behind student growth. ${ }^{7}$

Already in the 1990s, Gornitzka, Kyvik, and Larsen (1998) observed the relative growth of administrative positions at Norwegian universities, which they saw as contributing to changing expectations of what university administration should be. Keep in mind that this stunning expansion of a managerial class at universities over the last 45 years has occurred when most corporations around the world have decided to become 'lean', giving the impression that a very large workforce has moved from one sector to another, bringing with them their old management tools and models under the label of efficiency, while corporations moved in another direction.

In this chapter, we build on these observations to inquire further into our two universities' respective governance practices. There is no doubt that 
universities such as NTNU and UCLA face strong external forces that make them comply with neoliberal ideas about governance and the way universities shall provide public goods, including 'excellence'. However, what are the possibilities of implementing 'top-down' decisions in organizations with a strong tradition for local autonomy? We begin the chapter by discussing the hierarchical aspects of universities before we focus on academic freedom as factor that modifies external forces. In turn, this leads to an analysis of collegial organizing, using a constructivist perspective that we briefly introduced in Chapter 1.

We think that the critique of neoliberal governance of universities should be supplemented by an assessment of how local autonomy is practiced and with what consequences. We argue in this chapter that NTNU and UCLA through institutionalized forms of local as well as individual autonomy offer important spaces of self-governance that allow for resistance and resilience, but also for conflict and harassment. The conflicts usually are embedded in epistemic politics and factions through local competition for resources and recognition, as well as constructions of expertise and authority. We believe it is important to observe such spaces to be able to defend them, but also to understand how they might be improved.

The chapter develops this argument by drawing on the concepts of academic freedom, collegiality, factions, meritocracy, and academic citizenship, in the context of epistemic politics. While we observe how bureaucratizing and corporatizing circumscribe everyday life of universities, these interrelated concepts help us to observe important enactments and intersections related to local autonomy and the consequences for the enactments and circumscriptions of academic expertise and the academic work culture. At the same time, universities are hierarchal organizations with a wide array of hierarchical practices embedded in them. That is, to some extent, local academic hierarchies are the outcome of sedimented collegial practices and assessments. The relationships between academic freedom, university governance, and hierarchies are complex. This invites further scrutiny. In Chapter 4, we pursue these issues by studying processes of academic subject formation in the context of entrepreneurial universities.

\section{Universities as Hierarchies}

Many assume that academic governance takes place in a forum where many, if not all, would know the conventional ideas, discursive patterns, everyday procedures, and strategic moves that will be pursued there. The participants would have a rough familiarity with the alliances and factions, recruitment activities, rhetorical turns, and how people negotiate. They would agree on what constitutes heroic, mundane, venal, or selfless actions, if not who should receive those designations. That is not the case in contemporary academia. Many are aware of another kind of governance forum where people from 
many different cultures gather to adjudicate matters of concern, such as a United Nations or a trading zone at the interface of several cultures and economies. Typically, those participants would agree on a set of ground rules, such as diplomatic discourse and decorum, or at least a shared currency, within which they would discuss their differences and reach some agreement, however temporary or fragile. That also is not the case in academia.

During the last several decades, academia has become a much more turbulent space. It is as if a large assortment of people were using the same space, sometimes making use of or exchanging the same resources, but unaware of the different practices used by clusters of other people in the same space, cultivating a willed indifference to the existence of the others. In some cases, the awareness exists, but a stylized ignorance and silence is professed and taught, apparently as a way to refuse the slightest acknowledgment of the other governance options. Perhaps useful analogies are societies is which there is systemic ageism, racism, sexism, nationalism, ableism, and so on; people of one kind might be vaguely aware of the other kinds or make use of them, but the others do not matter at all. In such an arena what can be done? What cannot?

At both NTNU and UCLA there are a wide array of groups, each of which is very much engaged in building, maintaining, and reinforcing its own distinctive set of robust governance practices. Those groups might be engaged in academic capitalism, algorithmic corporatism, academic freedom initiatives, distinctive kinds of peer review, entrepreneurial individualism, regulatory administration, collaborative 'start-up' ventures, cronyism, bureaucratism, and so on. Some of these groups have been common on university campuses for a very long time; several have arrived more recently. Each of these clusters tends to focus either on a certain set of resources, some of which might be ignored by others, or particular stages in the allocation of those resources. Often, they will explicitly ignore the other groups' practices and concerns, in exchange for the group's autonomy. Sometimes they remain quite ignorant of other groups' expertise or actions. UCLA is a larger campus in many ways, so the range and size of these groups might be larger than at NTNU; scale might matter. Here we describe some of these practices and then address the consequences of the highly stylized, indifferent interactions among all those groups on campus.

Major universities usually include people from many different generational, gender, regional, class, ethnic, occupational, and organizational cultures from around the world. Those campuses are a cultural crossroads where we circulate ideas and resources from near and far. The customary practices of getting something done on a campus takes place within a rather fixed framework, often quite similar in universities around the world. The primary groupings are people clustered by kinds of expertise called disciplines with relatively rare mobility across the segments. The established disciplinary segments often are usually located in departments. Those kinds of departments compete with each other for resources and then unite to guard their traditional 
privileges against appropriation by the newer, emerging kinds of expertise, usually located in organizational sites called centers, programs, and institutes. Sometimes those marginal groups overcome the resistance and manage to 'departmentalize' and gain access to a 'line item' in the university budget, rather than subsisting on annual discretionary allotments.

On a daily basis, US universities are governed by two parallel authority structures: one is the organization of the professoriate, focused on teaching and research. The other authority structure is administrative; that one manages the university and all its bureaucratic processes. In practice that bifurcation is not firm and during the last few decades the teaching and research domains have become increasingly bureaucratized and many more administrative levels have been added to an already very hierarchical infrastructure. In addition to that twin framework, universities normally are saturated with extensive extramural, formal regulatory procedures as would any workplace. Both the academic and administrative formal procedures and customary practices have both informal and formal means of adjudication, revision, and redress. Some are strategic and some are not. At NTNU, there is formally only one authority structure, where the administration is subordinate to the academics employed as rector, deans, and heads of departments. Recently, this threelevel hierarchy has been supplemented by a fourth level that reports to heads of departments. This level is called sections or research groups; the authority of their leaders remains unclear. Not all departments have this fourth level; it depends on their size. NTNU departments range in size from 50 to 400 employees.

In US universities the academic hierarchy, in ascending order, begins with undergraduate (officially four years but often longer), then graduate students (five to ten years), culminating in doctoral degrees. Some become post-doctoral researchers, instructors, or lecturers, all of which are short-term appointments, perhaps renewable up to six years; there are a few universities, including some 'ivy league schools' that hire assistant professors on six-year terminal appointments. Sometimes those jobs are called 'folding chairs' in sharp contrast to the luxuries of the 'endowed chairs' offered to only a few full professors. Next in the US hierarchy are 'tenure track' assistant professors; in the US that position requires a doctoral degree. For the last 50 years typically 100-300 people from around the world apply for those jobs; during the same period an increasing percentage of the applicants have worked as postdocs, instructors, and lecturers before being hired as a faculty member. That lengthy process of awaiting a faculty appointment is referred to as 'being on the market', as in being a commodity awaiting consumption. It is a precarious condition. Once hired tenure track assistant professors in the US usually have a seven-year appointment; during their sixth year they face a year-long evaluation for promotion to tenure. At the top US universities about 30-50 percent of assistant professors are granted tenure and promoted to an associate professorship. Informally the process is described as 'up or out'. 
Typically, associate professors are eligible for promotion to a full professorship after seven years. However, in many US universities there are many long-term associate professors; most are women and members of other under-represented groups. A few full professors are granted special positions called 'chairs', endowed by private donors, which provide additional intramural funding to support various initiatives of the professor's choice. The entire process is structured by a series of required evaluations at specified intervals. However, that process can be accelerated and often is for those with powerful mentors.

The hierarchy of Norwegian universities is constructed differently. Students follow the so-called Bologna model, which is implemented in most European countries. They begin as bachelor students (should last three years), then master students (two years), and $\mathrm{PhD}$ students (three years). Norwegian authorities put a lot of emphasis on students fulfilling their degree within the allotted time frame, although many exceed it. The next level after the $\mathrm{PhD}$ is usually temporary positions, such as post-doctoral researchers, but the $\mathrm{PhD}$ formally qualifies for permanent employment (tenure) as an associate professor. However, being hired to such positions usually requires substantial additional publications beyond the dissertation, as well as teaching experience. Compared to the US situation, it is noticeable that the Norwegian government puts substantial pressure on universities to make them reduce temporary employment. Associate professors at Norwegian universities may apply for promotion to full professor whenever they assume that they are qualified. Qualifications are assessed through peer review by a committee with three members, two of whom should be from another university and at least one member from outside Norway. National guidelines inform the review process. At NTNU, nearly half of faculty are full professors.

In the US, university faculty members are hired by the faculty members in the department in which they will work. In Norway, the hiring process is managed by the faculty (what in the US would be called a division, composed of several departments) and the head of department, based on an assessment committee report. Faculty members have limited influence. This difference impacts departmental politics in US and Norwegian universities.

The age-grading of university hierarchies in both Norway and the US means that most students are young, and most professors are older. In the US in 1975, the age restrictions on admission to university undergraduate and graduate programs were declared illegal, leading to a rapid increase in the number of women and minority applicants. At about the same time fixed retirement ages became illegal in the US, except for very few occupations, such as being a surgeon, a firefighter, or an airline pilot. Subsequently, the same change occurred in Australia, Canada, and the UK. As in most wealthy countries, lifespan in the US doubled from 1920 to 2020, so many people remain active and healthy into their $80 \mathrm{~s}$. Although people can choose to retire at 65 , many who like their jobs do not. Consequently, the workforce in the 
US increasingly includes people 18-85 of multiple generational cohorts. In universities most people dispensing resources are over 50 and many with authority are older. In 2000 about one third of the US professoriate was over 60 years old; that proportion is increasing. The situation in Norway is similar, but the retirement age is fixed at 70 .

In sum, NTNU and UCLA are hierarchical, age-graded infrastructures segmented into clusters of expertise with rare mobility across segments. Leaders are chosen by those who are senior to them. Juniors are promoted by elders. However, arguably, universities have been hierarchical for centuries. Aside from some signs of increased informality, what has changed? At UCLA the hierarchy is greater than at the other UC campuses and continues to intensify. For example, the faculty committee with the greatest authority on campus, the Academic Senate's Committee on Privilege and Tenure, requires that only full professors be members, unlike the analogous committees on other UC campuses. Another difference concerns salary. While there are recommended salary scales for the UC system, for many years about 85 percent of UCLA faculty members have had 'off-scale' salaries. Periodically the office of the UC President has tried to curtail the difference, but that has not worked, yet. Within UCLA the pay gaps among faculty members at the same rank continue to increase. The gaps reveal different access to discretionary resources allocated to faculty members by their deans. ${ }^{8}$ The wage span at NTNU is narrower and subject to negotiations with faculty unions. There is no faculty union at UCLA, although there is a union for the post-doctoral researchers and some of the graduate students.

\section{Extramural Circulation}

Universities are not autonomous islands. The professoriate, as well as administrators, through the practice of their expertise, as teachers and researchers, have ties to colleagues in the same field at other universities nationally and abroad. Career advancement for professors and administrators in the US is often through moving to other universities. This is much less the case in Norway. The US-based administrators and faculty members who have moved among universities typically will have the most extensive, dense, and powerful meshworks and reputations beyond the university, ties that might be local, national, and international. Access to and participation in dense webs of relationships is based upon personal reputations for both quality work and reciprocity. Much is exchanged through those 'meshworks' which require active maintenance of reputations.

By contrast, those who do not circulate much beyond the home university, either as faculty administrators or as regular faculty members, might have acquired much local power, and developed intramural webs of relationships, but they often have little extramural influence. In the US there can be a very large gap in the scale of extramural relationships between those whose careers 
have moved beyond one university and those who have spent most of their careers at one place. Put differently, prestige usually is associated with circulation. (Of course, those with strong local ties would be reluctant to circulate, which is often the case for women and other under-represented groups.) In Norway, that distinction is not so vivid. A much larger percentage of faculty members will have spent most of their careers at the same university, often beginning as undergraduates. There will be a difference between those who have cultivated international relationships, as well as local and national ones, but prestige is not correlated with circulation.

Sharon has noticed in her circulations through universities in Europe, Japan, and the US that there usually is some stylized tension between the faculty members who have worked at more than one university and those who have not, as well as between those who have worked at universities outside their home countries or not (Pettersson, 2016). Even if academic mobility and international collaboration is encouraged by policy, and appears to be reinforced by prestige, in practice it can be resented strongly by those who have not moved or collaborated with extramural colleagues; they sometimes coalesce to push promotions of those like themselves. In Japan she heard one physicist criticize another as someone who 'reads too much sideways print'. By contrast, she also heard of cases in which a Japanese university department wanted to hire one of their own students, but others beyond that university coalesced to insist that a full evaluation of both extramural and internal candidates be conducted, resulting in the hiring of an extramural candidate.

In departments within highly ranked US universities, such as UCLA, the proportion of faculty members and graduate students raised outside the US can range from 10 to 75 percent. That wide variation suggests strong differences between department cultures about the internationalization of scholarship and academia. Because of its rather privileged global position, academics in the US sometimes believe that the best scholarship is conducted there; to counteract that clearly false thinking it is helpful to have more people from around the world working together daily. Global competition in academia clearly has reduced the number of US faculty positions held by those who have not circulated outside the US. Sharon has found the same cluster of issues being enacted in Japanese and Swedish universities and has heard much about similar concerns among academics in other countries when increasing numbers of very highly qualified foreigners begin to apply for faculty positions in departments where there have not been many. Meanwhile, the international university rankings continue to favor those departments and universities with more international representation. The universities most concerned with rankings will increase the international representation on their campuses and often will reward departments that do so. Nonetheless, ambivalence prevails.

So far, we have shown how universities are multiple, crosscutting hierarchies based on formal as well as informal criteria. Positions, prestige, 
reputation, expertise, and meshworks, both intra- and extramural, are all sources of power, most of which is allocated through hierarchies. This is the context within which universities are governed, collegial relations are shaped, academic freedom is enacted, and academic work is performed. In the next sections, we explore these practices more fully.

\section{Academic Freedom, Autonomy, and Epistemic Politics}

We both remember when 'academic freedom' primarily was invoked as a ceremonious concept on formal occasions. At the University of California those whose academic freedom had been violated years before, could be celebrated, such as Angela Davis who was fired by the UC Regents in 1969 from her position in the UCLA Philosophy Department, but became a Regents' Lecturer in Gender Studies at UCLA in 2014. ${ }^{9}$ However, in recent years academic freedom sometimes has become a strongly contested principle in the present, most often called upon in debates related to the enactment of freedom of speech in university contexts (Scott, 2019; Reichman, 2019). Freedom of speech is a right that all citizens have, although in present debates, this freedom is considered in particular ways when universities are in focus, often because freedom of teaching is at stake. However, here we are primarily interested in the institutionalization of the principle through higher education governance practices because this is a key to understand the basis of local autonomy and the development of local epistemic politics, including the nature of university governance.

The legal basis of the concept varies. In Norway, academic freedom is mandated through the Universities and University Colleges Act of 1968 and has been confirmed in the recent revision of the act. ${ }^{10}$ In the US, the legal basis is a Supreme Court decision from 1957 (Reichman 2019:10). This decision identifies four essential freedoms of a university, to determine who may teach, what may be taught, how it shall be taught, and who may be admitted to study. Thus, the main focus is teaching. However, in a broader sense, academic freedom should be understood in the context of Wilhelm von Humboldt's university ideal as an institution based on freedom of learning and research. This is clearly reflected in the proposition to the Norwegian Parliament to amend the Universities and University Colleges Act, which states that:

Academic freedom implies that universities and university colleges have the professional freedom themselves to shape their own professional and value-based foundation within the frames decided or supported by law. Furthermore, this indicates that they shall make sure that teaching, research and professional and artistic development maintain a high professional level and exercised in accordance with reputable, scientific, art professional, pedagogical and ethical principles. Academic freedom also implies that there cannot be given orders or instructions about teachings 
and the content of research or about the artistic and professional development work beyond the frames decided or supported by law. In addition, the individual employee of an institution subsidiary to the University and University Colleges Act an independent professional responsibility for content of and plans for the teaching. The individual employees also have the right to decide the topic of and plan for her/his research or development work, within the frames given by the employment contract or particular agreements. ${ }^{11}$

Thus, the proposition maintains the autonomy of academics with respect to research and teaching, both individually and institutionally. However, academic autonomy in the Norwegian context is relative to legal constraints that allow the government in general terms to regulate university affairs. However, the Act is very clear in stating that the content of teaching and research, as well as hiring and promotions, cannot be decided by the government.

The University of California has its own policy regarding academic freedom, which largely is similar to the Norwegian. It states that

'The principles of academic freedom protect freedom of inquiry and research, freedom of teaching, and freedom of expression and publication'. Legal constraints are not mentioned, but 'Academic freedom requires that teaching and scholarship be assessed by reference to the professional standards that sustain the University's pursuit and achievement of knowledge. The substance and nature of these standards properly lie within the expertise and authority of the faculty as a body'. ${ }^{12}$

Consequently, academic autonomy is relative, since neither faculty nor students can do, say, or write whatever they want while still considered to be 'proper' academics. Research and teaching should be shaped by professional and scholarly standards; they are at the center of local epistemic politics. As Joan Wallach Scott (2019) argues, academic work is disciplined in many ways. In professional contexts, to be accepted as academic performances, arguments must adhere to rules, standards, and tastes that are upheld through peer review and related collegial practices that assess and rank academic expertise. In addition, university bureaucracy confines academic work through administrative regulations and allocation of resources. Resource allocation is particularly important, since this may provide incentives to prioritize research topics that are high on the political agenda, such as those falling within the category of 'large social challenges'.

Both at NTNU and UCLA we find considerable autonomy exercised in decisions about the content of research and teaching, but confined by collegial preferences, assessments, and competition. Bureaucratic organizing practices impose formal standards and requirements about reporting and grant application work, but not with respect to subject matter other than at 
the level of study programs and course structure. However, funding agencies and external clients may exercise considerable influence regarding research topics, occasionally also with respect to research design. Thus, individual and institutional autonomy is relative and under pressure.

The main line of defense is the practice of collegial organizing that we introduced in Chapter 1. Collegial organizing is a set of processes related to decision making that is based on and upheld by the principles of academic freedom, but also a system that mediates this freedom through the practice of epistemic politics. It is important to explain that ambiguity. To begin with, 'collegial' sounds attractive because the concept signifies a friendly and democratic, face-to-face working relationship of people of equal standing and power that constitute a professional community, such as a department or a research center. Universities may be described as a community or a federation of such communities. Outside the US leaders traditionally were elected among the employees, who participated in the making of important decisions.

This way of organizing everyday academic work has been considered to be ideal for universities (Sahlin and Eriksson-Zetterquist, 2016). It seems to uphold academic freedom, while also providing for decisions to be grounded in academic expertise in a broad sense and in a deep knowledge of the local situation. Accordingly, Sahlin and Eriksson-Zetterquist describe collegial organization as resting on three pillars: formal structures for collegial decision making, elected leaders, and peer review. Then they observe that 'Collegiality is as much a culture of how work should be pursued ... primarily defined as a work process' (p. 3). This process we see as based on academic freedom but moderated by local epistemic politics. Sahlin and Eriksson-Zetterquist emphasize that the main rationale for a collegial system is that it is believed to produce high quality research and teaching, based on scientific, scholarly knowledge, arguments, and procedures. Thus, the system is assumed to be wiser and more competent in the relevant concrete decision making with respect to applying for grants, running projects, and publishing than its leaders. However, as we saw in Chapter 2, collegial systems have been intersected by changes in the political economy of the two universities. University governance has been transformed and hierarchies have been reproduced, while academic freedom as a principle has been retained. How does this shape collegial practices and everyday life at the universities?

\section{Transformations: Growth, Democracy, and Resilience}

According to the critical university studies literature, which we reviewed in Chapter 1, collegial systems have been eroded and subsumed by regimes where decision-making power has been centralized and coalesced into the hands of professional managers. In Chapter 2, we identified two such regimes at NTNU and UCLA that we termed bureaucratizing and corporatizing. The resulting multi-level, hierarchical decision making coincides with and is legitimized by 
a solidified belief in universities as strategic actors, a transformation argued to be necessary for making universities 'entrepreneurial' (Clark, 2004) and able to deal with changing political-economic contexts. However, this does not mean that collegial practices have disappeared.

There is no doubt that universities have faced comprehensive shifts in their political-economic contexts, which have changed their organizational and governance practices. However, there are three additional transformations that have been significant: (1) growth, (2) democratization, and (3) professionalization of research and research training. The most visible change is the enormous growth of universities, measured by the number of students, faculty, and staff but also in terms of budgets, buildings, and external relations. The number of universities worldwide and nationally has also grown tremendously for over 100 years (Frank and Meyer, 2007). These changes reflect the growing importance of higher education and research in modern societies, which has impacted nearly every kind of social activity. This explains the increased presence of government in university affairs, clearly demonstrated by the Norwegian developments described in Chapter 2.

The effect of size is often overlooked in ongoing discussions about universities. When Knut was a student, the university in Trondheim had 6500 students (1973) compared to 42,000 in 2019. The number of faculty has grown accordingly. This has changed fundamentally what it means to study and work at the university. Everyday life and the status of academics as well as the organization of university affairs have been transformed. For example, the small departments where we each started our careers have become much larger; to some extent due to mergers, a widespread phenomenon at our universities. This means that departments have become much less transparent and more difficult to 'know' in terms of knowledge about faculty and students, including teaching and research activities. In our experience, personal acquaintances beyond one's own department have declined, and the relationship to people in leadership positions is increasingly distant. The result is a more segmented and opaque university culture, which make internal meshworking and informal initiatives more demanding. In turn, this invites increased reliance on formal procedures and bureaucratizing. University life has definitively become more regulated, with more forms to complete, more applications to write, and more rules to follow.

Paradoxically, the second transformation - democratization - has also contributed to these developments. Historically, faculty's participation in decision making was an exclusive, elitist phenomenon. It was a privilege of the few who were full professors and allowed to elect their leaders among their own. Only those belonging to the most senior professoriate could take part in elections and formal decision making. Moreover, full professors had access to resources and the power to decide about research, teaching, assessments, and hiring. Thus, they dominated local epistemic politics. These privileges were first beginning to be undone due to student activism in the 1960s and 1970s. 
Student activism had many objectives and the emphasis varied considerably from place to place. A shared perspective was making universities more democratic in the sense that the might of full professors should be dismantled. An important goal was that participation in decision-making bodies and elections of leaders should be made representative of the university as a whole. This should include not only students, but also temporary faculty and administrative employees. Over time, largely, these demands have been met in Norway. At UCLA, full professors have retained some positional power and leadership positions are not elected. (As mentioned earlier, at UCLA it remains the case that only the most senior professors can serve on the most important faculty committee on campus.) Students and staff have little or no voice in governance. Knut has found that some NTNU professors recruited from other countries express surprise when they discover they have little such influence in the Norwegian context. Sharon has observed in Japan that foreign full professors from Germany and the US have found their day-to-day control to be far less than they would have in their home countries.

In recent complaints about the demise of collegial practices, the benefits from the dismantling of some of the power of the professoriate tend to be forgotten. If professors have lost some influence, this was not primarily due to the introduction of business-like management practices. In Europe, the changes came earlier and were also rooted in the formal, representation-based democratization of universities. However, over time, this democratization has reinforced the effects of the growth of the universities to increasingly formalize decision making and to bureaucratize university life. The informal way of settling issues that was predominant and feasible at small departments has increasingly been replaced by more formal procedures because large departments face many more decisions than small ones, and many more people could in principle be involved.

Thus, the vastly increased number of students, faculty, and staff has partly been accommodated by systems of representation. At the same time, the decision-making power of deans and heads of departments has increased considerably, explained by a claimed need to make decision making more efficient. UCLA has retained a system with departmental faculty meetings that make important decisions, for example regarding tenure and promotions, but they can be over-ruled. In the larger departments, decision making is located in elected 'executive committees' and the full faculty meetings tend to be filled with announcements of recent decisions made elsewhere.

NTNU has a system of department boards with limited decision-making power. As already mentioned, this has increased the distance to decision making, which is experienced as a loss of involvement (Ingelsrud and Falkum, 2019). What originally was intended as a democratizing reform has contributed to producing an opaque, bureaucratic organism, which circumscribes collegial ways of organizing academic work. Thus, democratization has proved to be an elusive concept, one may argue that a democratic 
governance of universities should go beyond workplace democracy to give the wider public some influence. This has not happened, although increasingly so-called external representatives have been appointed to the boards. In Norway, this is required by law, and the representatives are appointed by the Ministry of Research and Education.

The governance system at UCLA is more complex, since UCLA is part of the University of California (UC) system, which in principle is subject to the State of California. In practice, the UC system is governed by a Board of Regents, meant to represent the public. They are appointed by the governor; they provide links to social groups that are considered important to the running of universities, such as industry and the public sector. Many are prominent businesspeople who think the university would best be managed as a business. Often, such governing boards have strongly advocated corporatizing initiatives.

The third transformation is the increased professionalization of research management and research training. By professionalization we mean the introduction and development of formal procedures that has furthered the bureaucratizing of the universities. This includes the rising demand for more detailed reporting, but also growing requirements of administrative involvement in grant application and project management. Previously, we could work on grant applications until the deadline and calculate expenditures on our own. Now, budgeting requires accounting expertise due to a complex set of new rules and systems, and the administration demands to be involved in quality control of grant applications. Thus, the work with applications must start much earlier and involves more effort than previously. There often is a presumption that there should be extensive oversight of the ethics of faculty members regarding both budgetary matters and research design. We will return to the issue of research training and the changes in the processes of becoming an academic in the next chapter.

\section{Corporatizing and Bureaucratizing as Circumscriptions of Academic Life}

We addressed some of the issues related to governance of universities in Chapter 2, highlighting the role of bureaucratizing and corporatizing. We also emphasized how the strong presence of the government in the case of NTNU resulted in a pressure of increased bureaucratizing. So far in this chapter, we have been concerned with academic freedom that we consider as an important, but often overlooked organizing principle. How does this relate to corporatizing and bureaucratizing? Undeniably, collegial organizing of work has been encroached by increasing bureaucratizing. This is evident from the growth of formal procedures for work and study and the increased demand for more reports and metrics to assess achievements. As noticed above, there has been an increase in the number of administrative positions; 
that increase is vast at UCLA. Judged from available documents, bureaucratizing has been justified by concerns for efficiency, harmonization of rules and practices across the universities, but also by the wish to control the performance of teaching, research, and outreach. As we noted in Chapter 2, such efforts are meant to make the universities more transparent and accountable. The increased emphasis on students' rights has also been an effectful driver at NTNU. The related bureaucratizing admittedly has some progressive, but ambiguous effects. Presumably, students experience improvements that to faculty mean an increased workload.

At NTNU bureaucratizing has been more effective in changing the everyday life of university faculty than the business-inspired corporatizing that has been the focus of much critical literature on the development of higher education. However, the two forces are not mutually exclusive. At UCLA many of the bureaucratic forms and procedures have been taken from 1980s and 1990s corporate models and implemented via corporate management software and outsourcing contracts to private companies. Bureaucratizing has shaped research and teaching comprehensively through the disciplining efforts that have increased homogeneity and governability. This has had a tangible influence on everyday life at NTNU and UCLA because the efforts clearly intersect with teaching and research practices and thus interfere with collegial ways of organizing these activities. For example, we observe new requirements about teacher student interaction, about the procedures to assess teaching quality, about assessment of students, about simple things such as travel expense claims, access to office equipment, installation of software, and reports of professional achievements.

Thus, bureaucratizing changes some academic practices at a very detailed level, but it does not replace collegial organizing. Rather, to some extent the efforts may counter some weaknesses with collegial ways, such as dealing with teaching quality and coordination. There are also benefits in terms of transparency with respect to the distribution of teaching duties and service work among faculty. This may increase fairness in the distribution of responsibilities and benefits. Fairness is potentially an Achilles' heel of collegial organizing.

We use the term corporatizing to describe practices mainly exercised by upper-level university leadership dealing with so-called strategic decision making. The practices are clearly inspired by ideas from business administration and industrial management of the last century that above all highlight the importance of leaders and strategic leadership. The corporatizing discourses also focus on financial issues to make university leadership sensitive to 'business opportunities'. However, as we observed in the previous chapter, these discourses are understood and implemented rather differently at NTNU and UCLA.

At NTNU, corporatizing is visible largely as a rhetorical device to provide a measure of accountability of the university to outsiders such as policy 
makers, politicians, industry, funding institutions, and the public. A major aspect is to make the top-level leadership appear competent and up-to-date with respect to business management approaches. At NTNU, as discussed in Chapter 2, this appears somewhat paradoxically in strategy discourses based on a perverted use of so-called 'management by objectives', promoted by Peter Drucker in the early 1950s. The objectives have been shaped by the morphing of excellence ideology in the strategy plans, resulting in moderated ambitions. Most of all, as noticed, the objectives reflect the requirements of the Ministry of Research and Education, translated into marketing prose, intersected by pictures of engaged and good-looking young people. Since NTNU has limited opportunities to change its operations to pursue economic opportunities beyond research funding, corporatizing appears largely as efforts of steering with 'big words' (Bos et al., 2014). The top leadership presents a broad spectrum of ambitions, referring to 'our owner' and 'our social mission'. 'Excellence' is seldom used and in case, with considerable care. This may produce a sort of academic governmentality, the understanding that it is 'normal' to pursue the stated ambitions. However, it is unclear how effective this governmentality is, given that many academics shrug at and joke about 'big words'. This reflects the imagined features of the university that we touched upon in Chapter 1, the game of make believe that the university is controlled by the top leadership.

Corporatizing exercises greater influence in its shaping of the systems of internal distribution of resources through incentive systems. There is also competitive allocation of resources for some purposes, such as improving teaching methods, or research in a few so-called focus areas, for example 'emerging technologies'. To be effective, incentive systems need to be accompanied by information about performances. NTNU has a designated system for such information, called BEVISST (CONSCIOUS), to emphasize that performance management should be informed.

As leader and leadership support, through BEVISST insight you will gain a better understanding of activity areas such as teaching, research, dissemination, and innovation, and the support functions economy and human relations. BEVISST plan shall make you able as leader and leadership support to resource plan operations and assess the realism of plans and budgets for as long as 10 years into the future. ${ }^{13}$

The scope of the system is impressive, and it clearly demonstrates how a wide variety of academic performances may be measured and calculated to give deans and heads of departments a comparative overview of achievements as a tool for planning and control. Thus, at first glance, BEVISST looks like a Panopticon, a system where the center sees everything while the parts only may observe (and send data to) the center. According to Foucault (1977), panopticon-like constructions are effective disciplining devices. However, 
most faculty are blissfully ignorant of the system's existence, and it contains only department-level information. Moreover, the access to the system is restricted. It can only be used by leaders and administrators designated as 'leadership support'.

The UCLA Provost's Office is in the midst of implementing a new organizational model, called 'hub and spoke'. The model is identified as one of UCLA's six organizational 'strategic priorities', along with the New Campus Budget Model, Administrative Reorganization, Sustainable Operations, Processes and Personnel, and Revenue Generation, all based on existing platforms developed long ago in the corporate world. Closer inspection reveals that the hub and spoke model interestingly leaves out the wheel rim. Consequently, all information and communication focus on the hub at the expense of building a robust and resourceful array greater than the sum. The general tone of the information on the hub and spoke model provided by the UCLA administration to faculty and staff is simplified and vague. One example: "Hub and Spoke" is a way of linking the hiring, training, and supervision of local staff (the "spokes," including locally-run service centers) to organizations at the center (the "hubs")'. ${ }^{14}$

Although it claims the model is not hierarchical, the scholarly literature states that is its defining feature. Hub and spoke organizational models have existed since the 1950s beginning in two fields: transport (and later service) distribution industries and the other in cold war diplomacy, exemplified by the US bilateral ties with several Asian countries, as opposed to the multilateral relations that the US and Europe countries have cultivated since the 1949 establishment of NATO. Another study showed that the British foreign office long used a hub and spoke structure that worked against the emergence of communities of practice among its offices in various countries (Venters and Wood, 2007). Another study showed that the Occupy Wall Street (OWS) protesters who communicated via Twitter found that the technology had hub and spoke consequences (several central users distributed information to a loosely connected hierarchical network), while those using YouTube videos generated a dense meshwork, reinforcing shared ideas and meanings among the OWS members (Park, Lim and Park, 2015). Many corporations and government agencies stopped using hub and spoke models due to their inherent weakness: It is highly centralized; failure at the hub leads to system collapse.

The main idea behind NTNU's centralized BEVISST system is to provide leaders with information from many systems, simplifying the overview of the economy and performance of departments. Faculty may gain information from their dean or head of department, usually through designated meetings. The information provided, tends to be selective, mediated, and adapted to highlight successes or concerns considered relevant by the leadership. Information about individual performances is available from other databases and reports, but NTNU has no formal system for individual assessments other than promotions, which are triggered through application. 
Thus, academic performance by individuals or departments is seldom made into a public issue at NTNU. This limits corporatizing efforts. NTNU's Rector ritually congratulates faculty with their achievements at the end of the calendar year. Some departments have been told at internal meetings that they need to do better with respect to external funding, but such complaints tend to be countered by faculty explaining that funding is difficult to achieve in their professional area. Thus, the complaint is discarded as unreasonable. The governmentality emanating from corporatizing discourses at NTNU appears to be restricted.

The widespread concerns about the erosion of collegial organizing have focused on changes in faculty members' participation in formal decision making, but there are also worries with respect to the autonomy of everyday academic work. The Covid-19 pandemic has added to these qualms because in many universities, temporary university positions, even tenured professorships, have become more precarious. These concerns are serious, and often the precariat is reluctant to assert any privileges. However, it is important to remember that the reproduction of academic freedom and autonomy feeds on the efforts and struggles to make use of the autonomy. What we see is that academic autonomy resides both in beliefs and in practices; it depends on enactment by individuals and clusters of people making decisions about the conditions of their work. We do find that such collegial practices endure, evidence of the performativity of the idea of academic freedom. Ironically, these practices are also upheld because they are cost effective, resulting in a low density of leaders at the departmental level, compared to many other types of organizations. However, what is involved in the informal enactments of collegiality? How may we understand decision making in this context?

\section{Decision making with factions}

We have described how decision making at NTNU and UCLA is shaped by the hierarchical, age-graded segmentation into departments that include faculty members engaged in lower to mid-level administration, alongside those focused on teaching and research. This group coordinates the day-today work of teaching and coordination of resources for the work of faculty members and students. Superimposed on the departments is another apparatus that manages the campus and its extramural affairs. At NTNU that twopart structure is overseen by a powerful extramural administration from the national government. In the US there is no such government agency.

There is another feature that has a strong effect on decision making: our systems are characterized by considerable factionalization, albeit to a varying degree. Sharon learned from one of her dissertation advisers, Triloki Nath Pandey, a specialist on factionalized societies, that such infrastructures form around access to the discretionary allocation of resources, dispensed by a few (Pandey 1967, 1968). For a very long time the UC system has distributed 
discretionary resources, including salaries, throughout the administrative apparatus, creating extensive webs of patronage allocated through factions. Every UC campus and every department with which Sharon is familiar has been saturated with factions, along with the usual patrons and clients. Knut's experience from NTNU is that many departments have factions, but there are fewer discretionary resources available in the system. This erodes the potential power base of factions. In turn, that difference between NTNU and UCLA has important and interesting consequences.

In order to be promoted beyond their current positions, upper-level administrators must show that they have achieved something distinctive. Upon taking office each of them specifies some 'mission' or 'initiative' that they then plan to accomplish in 3-5 years. The chancellors, provosts, deans, and in some cases department chairs all receive resources they can dispense on their own authority to accomplish their mission statements. The nature and scale of the resources depend upon each administrator's rank and negotiating skills in dealing with their supervisors. At NTNU, mission statements tend to reflect government policies, and the resources that administrators have available is fairly small, in particular for heads of departments.

At UCLA, the resources each administrator can distribute are not transparent, nor is the distribution process. The administrators will use their resources to accomplish their stated initiatives. They also build webs of patronage among those whose goals intersect with the administrator. Within a department the resources might seem rather paltry to an outsider, but when not all the resources to do a job well are made available routinely, access to scarce resources might strongly affect the quality of life and work on an everyday basis: the scheduling and location of classes, access to teaching certain courses, support for teaching assistants, a newer computer, and so on. As the university pushes for increased international rankings and greater performance metrics from departments and faculty members, competition for the scarce resources increase. The resources are being allocated through increasingly hierarchical and factionalized governance. Even attention from a power broker becomes another scarce resource to be found strategically.

In her first faculty position at MIT, Sharon gradually noticed that in the faculty lunchroom there was a certain 'elbowing' among the junior faculty attempting to join certain senior faculty members at certain tables. Usually each of the tables was occupied by a full professor, along with their normal cluster of postdocs and graduate students. The department chair normally sat at a table with the 'endowed' chairs. Sharon routinely took a place at the least occupied table. After some months at MIT, the department chair asked Sharon to schedule a meeting with him which she did. He asked about her teaching and research progress and she told him about that. He listened for a while and then interrupted to say that she seemed to be doing fine. He added, 'Why haven't I heard about this before now?' He said that Sharon should keep him posted through informal chats. She asked if he meant she should be 
'elbowing' the others in the lunchroom as she had been witnessing; he laughed and said yes. Sharon asked if instead she could just send him reports or meet with him once a semester. He agreed, but said she should learn to do some 'elbowing' and Sharon said she would rather not.

The faculty meetings there followed a routine she has seen at other departments and universities since then. If one person of a certain rank spoke for five minutes, then everyone else at the same rank predictably spoke for five minutes. The people with the most and the least power said very little. Gradually Sharon realized that very little was being decided at most meetings, but much was announced by some and discussed by yet others. When asked to serve on a MIT university committee as a department delegate, she asked if the committee had any decision-making power; her chair told her he was very surprised she felt entitled to ask such a question. He seemed both annoyed and intrigued. She said she was following the advice of a mentor. Knut's experience has also been that little is decided at faculty meetings. Increasingly, at NTNU such events have disappeared; occasionally replaced by open meetings where also PhD students and temporary researchers may participate. Such meetings are mainly for exchanges of information, usually dominated by department leadership.

Joining local factions may be necessary for access to local power. However, in academia such access is definitely not necessary to have a significant reputation beyond the local site, so it is easy to find people in academia who actively avoid all local forms of power, including access to any local resources. Still, joining local factions has major consequences within and beyond the local environment, as does not joining factions. They are led by senior faculty members and include people at all ranks, even graduate students. They interact with those in other factions only in stylized ways. Factions usually maintain their differences from the others. It is easy to witness some of these routine activities at department events, such as colloquia. Motivations for joining local factions vary. Some do not realize that local factions often have little influence beyond the local environment. In the UCLA ecology where factions are pervasive, it is more unusual to avoid factions, but not rare. Some are suspicious of those who are not members; most just ignore the outliers. It is difficult to contribute to the everyday work of departments or the university when most of that work is managed informally by factions and decided by their leaders.

Sharon has spoken to several people in university administration about governance through factions, including a former president of the UC system. They all have seen the factions as predating their own position in governance and likely to continue long afterward. If factions strongly correlate with the non-transparent allocation of scarce resources at the discretion of individual administrators, the only solution seems to be ending that way of defining (and funding) leadership. However, over the last few decades it has been intensifying, making the system unsustainable in the long run, a topic 
we will address in Chapter 6. By contrast, NTNU only allocates insubstantial resources through leaders since the main part of the budget is allocated through models based on university performance indicators and previous allocations. This was described in more detail in Chapter 2.

\section{Factions, Conflicts, and Receivership}

There are two other troublesome byproducts of a factionalized ecology. The first is that factions compete, often in messy ways, and the competition can escalate. Competition may also be about recognition and status or about professional standards and curricula. This may make a university department unmanageable and a hostile place to work. When department members cannot agree enough to fulfill their basic governance responsibilities and retain a reasonable work environment, this may lead to external intervention. At NTNU, university leadership and administration tend to show considerable restraint in intervening in what some factions insist on calling professional disagreements. Professional disputes are a normal feature of academic life and often an important resource of professional development of research and teaching (Latour, 1987). Usually, academic communities have learned to live with disputes, accepting that there are disagreements and competition, which may be managed through collegial recognition of differences and condoning decisions that are made in proper fashion, even if one disagrees with them.

However, occasionally, departments have factions that are unable to manage their disputes without turning them into conflicts. Recently, it has become publicly known that three departments at NTNU have struggled with internal fights to the extent that several faculty members were reported sick at one of them and that students made strong complaints about the quality of teaching at one of the others. In one case, it took several years before university leadership and administration intervened and eventually decided to split the department in two parts. With the two other departments, it is still unclear what will happen.

Such conflicts illustrate a problematic aspect of academic freedom as a pillar of collegial organizing; it may allow for unhealthy power games and harassment. Usually, according to our observations, such practices are moderated and to some extent contained. Unlike NTNU, UCLA has a formalized practice of regularly reviewing departments where so-called academic receivership is a potential and thus a disciplining outcome. Academic receivership means that the control of an academic department or program is removed from its own faculty members and an outside chair is put in place by the college or the university administration (Stone, 2009). The official term of 'academic receivership' is not the same as 'financial receivership'. UCLA has a website that lists the normal departmental review process; the last three items identify 'special actions', one of which is receivership and one identifies the actions to be taken if receivership is unsuccessful. ${ }^{15}$ The consequences of unsuccessful 
receivership include splitting the department, forced merger with another department, and dissolution. However, the factions usually are reproduced.

There are extensive instances of receivership in US universities, but few studies. Sharon recalls hearing of several departmental receiverships over the decades, plus at least six departmental dissolutions, three departments having split, and three departments having been merged, all forced by university administrations. In public, all those cases were represented as either merging groups with very similar intellectual commitments or separating very different research subfields, each with stronger intellectual ties to other departments' disciplines. Informally the issues of factions and bullying were discussed widely. Sharon has heard about three cases of 'threatened receivership' at UCLA; each led to a series of required mediation processes. She was on research leave during one of those required mediation periods. The first day she returned to campus, Sharon was surprised when a colleague who barely knew her and had ignored her for years greeted her in the corridor. Later Sharon learned that everyone had been told that being 'cordial' was required and an example of being cordial was to acknowledge each other when in a shared space.

In another department Sharon was present during the required mediation sessions and was startled when she briefly became the subject of some criticism that seemed off-target; it quickly ended when the perpetrator suddenly realized Sharon was not a part of the faction that the accuser opposed. She had 'forgotten' Sharon was not in any faction. The last several years Sharon has experienced students seeking her counsel when they decide to leave factions or are pushed out. They know her reputation for being an outsider who somehow survives. In turn, Sharon has learned much about the anxieties of the students who have thought being in a faction was a necessary part of graduate school. A related issue is faculty members in one department wanting to move to another department. In addition to her own experience, Sharon is aware of about 15 faculty members at UCLA who have changed their departmental affiliations, as well as about 30 people who maintain multiple departmental affiliations. A colleague at another highly factionalized, prominent university once cheerfully described how he had managed to become affiliated with four departments. He said each one forgets he is a member, enabling him to avoid the extensive conflicts in each.

\section{Factions and Pawa Hara}

Another consequence of factionalism is the isolation of those who do not join. Those without patronage are subject to bullying, done partly to demonstrate to others that they should join a faction, not only for access to resources, but also for protection. We have different experiences with harassment, which reflects differences between the local political economies and university cultures. Competition is definitively a feature at Norwegian 
universities, but the competitive practices at leading US universities such as UCLA are harsher and more pervasive. Our experiences reflect this but also our dissimilar positions at our institutions, Knut as an insider, Sharon as an outsider. The following excerpt from Sharon's fieldnotes show some of the consequences of being an outsider at US universities.

First, I hasten to interject that the issues I describe here and elsewhere in this book were selected because I have learned how typical they are. That is, they represent routine practices rather than instances of unique, distinctively personal interactions. As noted in the discussion about autoethnography in Chapter 1, we participant observers study our own interactions in the 'field' to learn more about sense-making in a community, how decision making is conducted, and so on. Next, I want to introduce a Japanese expression, pawa hara or power harassment. It refers to any abuse of authority, from sexual harassment to bullying and scorning, by someone in authority against someone who is not.

I have felt bullied for a long time in academia, but not always. In retrospect it began about the same time my work began gaining some extramural recognition beyond that normally expected, enabling me to get access to more extramural resources, such as funding for my research. Gradually I realized I was immersed in local worlds of intense competition for scarce resources. I was aware of the old clichés about academia as a place where the nastiness increased with the diminishing size of the rewards. When I began to be seen as having resources that I might allocate locally, some treated me as a potential benefactor and others saw my resources as something they should and could capture. I had witnessed efforts to capture others' local resources since I had been in graduate school. From those experiences I gradually learned much about what is seen as power within universities and how to get it. For our current project I ask what kind of bullying happens on campuses and has it changed with the 'quest for excellence' and enhanced reputations?

Initially I experienced the bullying as rudeness coming from people I was able to ignore. In my first week as an assistant professor at MIT a woman stopped me in the hallway near my office, introduced herself, and asked me who I knew. I did not understand the question, nor the blunt way it was delivered. She explained by saying I had a PhD from 'a nothing place' (UCSC) so I must have gotten my job through connections; she wanted to know who it was. I responded that I did not know, so she must ask the people who hired me. Another time the rudeness came from someone senior to me. I got funding to conduct research that required I be granted a temporary "leave of absence without pay' from my position, a request that I had thought was perfunctory, based upon my all my prior experience in academia. (Typically a recent $\mathrm{PhD}$ who had not yet secured a faculty position would be hired to teach the faculty member's courses during their absence.) My mentor told me that my request would be refused because I had 'to wait my turn' for that local resource. I then realized that everyone senior to me had had more research leaves than I had; 
the rest of us had had fewer leaves. I had not noticed the difference as a barrier I was not (yet) allowed to cross. I was stunned, reflected on the situation, and decided to begin seeking work elsewhere, and eventually left. A few years later I learned my former mentor had spread some untrue stories about me to explain the reason for my departure, never mentioning that I had been denied the privilege of having as much research leave as the top half of the department's members.

Several months after I got to UCLA, I began to feel especially tired at the end of days I spent on campus. Eventually I noticed that a cluster of people were consistently being rude to me in what seemed to be rather random, impersonal ways. Obliquely, I asked a colleague about the people involved. She explained that it concerned power: in that very large department there were a set of highly sought-after administrative positions; those holding the more powerful jobs appointed those more junior. My colleague said that I was attracting the attention of the informal powerful group running the department, which annoyed those seeking their attention. She added that when the elders were watching, the annoyed would try to put me in a bad light. As part of being hired at UCLA I had been offered some resources to build links within the department and across the university in my research field. I noticed that in both meetings on campus and departmental social events some peers would make casually rude remarks about my research specialty. At a cocktail party I briefly mentioned an interesting conference I had attended recently. With a big grin someone asked: 'Sharon, why would anyone be interested in [that subject]?' While in a campus restroom, a university administrator I only slightly knew said I should know that one of my colleagues was spreading negative stories about me, while asking how he could get the resources allotted to me, transferred to him. Once one of my prior mentors who had become quite influential internationally was visiting UCLA and gave a lecture in one of the grander venues; I was sitting in the back of the large room next to some colleagues. The visitor made some opening remarks, thanking his hosts and added how pleased he was to see me. A senior colleague next to me whispered, 'Did you sleep with him, too?' Later I learned that another senior colleague had been regaling colleagues at dinner parties about my alleged promiscuity. That explained some shunning I had experienced from some people I had known who were rather conservative socially. At first, I could not understand why any of those senior colleagues had an interest in my social life, which in fact was quite mundane. Then I realized that, just as at my prior university, I was being seen as someone presuming to move 'up' into their privileged local domain, but they were demonstrating that I was not one of them, at least yet. My extramural resources were routinely demeaned and the intramural ones were seen as available for capture.

I asked an array of senior colleagues in other fields and at other universities how I should respond to all that. They said the behavior was normal and to be expected whenever we begin to get resources on campus. They all gave the 
same advice: 'kneecap them'. When I asked for another strategy, they all said that was the only option. Later I told each of them I had abandoned all the intramural resources; they all were aghast. I said I had learned to never seek any campus resources of any kind. I would only seek extramural funding for my research. I was not interested in that elbowing or kneecapping, a very embodied form of gaining power through symbolic violence. I learned that to refuse both domination and submission is considered an affront to the increasingly competitive, hierarchical, factionalized apparatus at UCLA. I had worked at being ignored on campus, but was not succeeding. UCLA seeks a greater reputation and wants to acquire those whose reputations are rising, as it had done with me; I was recruited with resources. However, I was seen as a problem among the locals who had honed their routines for accessing scarce intramural resources through years of working within an increasingly hierarchical, highly factionalized university that allocates important resources everyday through discretionary means under a metric veneer. They were demonstrating that I was not (yet) one of them.

One day while walking across campus, an informal mentor who was a very senior university administrator asked me to help him by joining a special digital teaching project at the last minute; I was interested and agreed. The next day while walking near my department's offices someone in the governing circle began shouting at me about doing something without permission. I just walked away and went to the administrator's office in another building and asked what I should do; he said I should do nothing, but he would. The next day the shouter gave me a friendly hello, but no apology. The change in his pawa hara displays were not for my benefit. What infuriated him? I had gotten access to a scarce resource, however trivial, that was allocated by someone far senior to him without immediately consulting the department's governing circle. At the time (2010) I thought the department leaders could care less about me being a substitute teacher for an undergraduate class with digital access to Japan. The shouter thought I had ignored the group that ran the department, unacceptable in the context of endless reputational negotiations. I merely wanted them to ignore me; I wanted some autonomy.

It is interesting to see what newcomers do when they witness one of the ritual pawa hara displays against the marginalized, which includes me, among others. Of course, the typical response is to shun the marginalized, displaying submission to the power harassers. Later, when some faculty members or students have sensed they are becoming marginalized, they then remember me and my strategic survival in the margins; they want to talk. We share the negative stories we have heard about each other from the power harassers who appear to not be aware of either the communications among those of us at the edge or our supporters. In many ways we marginalized do not have much in common, demographically, generationally, or epistemically, and the bullying we experience, no matter how intense or trivial, never seems particularly personal. Instead, we seem to have been bullied as a display of control, 
or rather a punishment for not having been controlled. What we share is being outliers to the factions. I have noticed in both Japan and the US that those at the edge of a group, not fully assimilated, often are selected as the object of pawa hara displays. Some who are seeking power will imitate the pawa hara displays of the more powerful, selecting the same marginalized people. Studies of bullying internationally show the same features: bullying is not about the ritually scorned bullied. Everyone learns the consequences of being in the margins; some begin to reflect on what they see. It is an excellent location from which to observe the construction and practice of local power, as noted by many social theorists, from Marx and Derrida to Anzaldua and Sandoval.

In my fieldwork among physicists and astronomers I sometimes notice the same processes at work. Newcomers learn the limits of acceptable behavior and thinking by noting the ritual pawa hara of those at the edge. The marginalized are not outside the community but mark its boundaries; we have an important function, outlining the borderlands. Of course, the most powerful are those who have not only learned to practice pawa hara, but also have become aware of its limits. They sometimes will have conversations about those practices with those of us at the edge, knowing that we, too, understand very well how the system works. They certainly are aware that there is another kind of power at the edge. They want their teams and their labs to be at the cutting edge, and that takes risk. They are intrigued by those of us who are willing to risk being at the edge of a knowledge-making community and unwilling to either lead or submit to factions.

Of course, factions exist in any system that allocates resources through discretionary means. Pawa hara is an ancient practice. What is it about these situations that locate them in the current academic formations? In each case I was accessing resources newly designated for resolutely interdisciplinary projects within arenas controlled by either traditional disciplines or those building disciplines. Those interdisciplinary projects were beginning to get the kind of resources that had been the province of either the existing or emerging disciplines. At the edge of the disciplines are many eager to build new ones; some of us are not interested. We return to that topic in Chapter 5.

\section{Enactments of and Limits to Academic Freedom}

Ideal forms of collegial, democratic organization never dominated universities. Existing collegial relations may be friendly, but also strained, conflictual, hierarchical, and harassing. Competition and power struggles are too often played out in the local epistemic politics. Thus, as demonstrated by Sharon's account above, we need critical reflection about how collegiality and academic freedom are enacted and how enactments might improve, acknowledging their ambiguous features. Academic autonomy may facilitate harassment, but it also allows a kind of self-management at work that many of us 
highly appreciate. We believe that academic work, at least to some extent, takes place in partly protected spaces that facilitated resistance toward neoliberal reforms; niches where we may find seeds of ways to organize universities other than the neoliberal template. What constructive practices have endured, despite bureaucratizing and corporatizing? Is it possible to find more beneficial, non-harassing practices of local epistemic politics?

Consider the everyday life of a university academic. If we shadow faculty, we quickly observe how work is shaped by the different temporal requirements of teaching and research. Teaching, including preparation and follow-ups, is done largely according to set schedules. These schedules are usually beyond the control of the individual academic, although the structuring and content of teaching efforts allow some freedom. There are limited instructions about how teaching should be done. NTNU has mandatory pedagogical courses, but Sharon has never encountered one in any US university. Nobody reminds us about when or where to lecture.

Research is different. Temporal shaping may occur in the form of deadlines, meetings, workshops, and conferences, but research is usually a flexible activity that may be done at any time, if access to labs and equipment is flexible. To some extent, teaching schedules override research since their temporality is stricter. Therefore, faculty often complain about lack of time to do research because teaching commitments periodically only allow for intermittent research efforts (Sørensen and Moratti, 2019). Still, research is largely selfmanaged, more so than teaching, mainly limited by considerations related to collaboration with other people. This is a mixed blessing, since this freedom also provides a temptation to work more and to do so outside normal hours of work. Moreover, the different temporalities of research and teaching may explain why many academics talk about teaching obligations, but rarely about research duties.

In Norway, in legal rules regarding work-life environment, faculty are classified as having a particularly independent position. At least in principle, this means that they decide what to do, when it shall be done, how it shall be done, and what may be delegated to others - in other words that they are selfmanaged. One formal consequence is that university academics are excepted from rules regarding hours of work. For example, they are not compensated for overtime. The concept of scholarly independence is used for praise, most often in the humanities and the social sciences. It signifies the ability to conduct research individually. However, when faculty members talk about our work, we seldom use such terms as independence, autonomy, and freedom. Rather, we emphasize being busy, having too much to do, and the need for hard work. However, when confronted with long hours of work and relatively low wages, a common response is 'I appreciate the freedom in my work'.

The issue of collegial organizing concerns different modes of influence and participation in decision making. We may explore this by drawing on Thorsrud and Emery's (1970) account of the large-scale Norwegian experiments in 
industrial democracy in the 1960s. They usefully distinguish between formal systems of workers' representation on boards of directors on the one hand and workers' participation in decisions directly related to their work on the other. At both NTNU and UCLA, faculty members have retained some formal representation in decision-making boards. However, the experience of being part of formal decision making has declined more strongly than the actual representation, since the massive growth in the number of students and faculty means that only a very small minority serve as board members and most of the university population do not know anybody who serves.

However, we want to focus more on collegial organizing enacted through our participation in decision making in everyday academic work, in line with the second aspect of the Norwegian experiments in industrial democracy. Consequently, collegial organizing needs to be understood with less emphasis on formal participation and election of leaders. Rather, we pursue Sahlin and Eriksson-Zetterquist's (2016) stress on work culture. At the heart of the issue is the concept of academic freedom. What kind of everyday life practices constitute our relative academic autonomy? This is a complex issue also with respect to a given university, since such practices vary across departments, research groups, positions, and individuals. We have seen how some departments try to reduce this autonomy, for example through prioritizing certain research areas or forms of teaching, while other departments are more relaxed. Still, self-management is a predominant practice, meaning that to a large extent, faculty members individually and/or through collegial collaboration decide how they conduct their work.

In fact, our universities largely rely on self-management. This is evident from the low proportion of leaders relative to the number of faculty. The exact number is difficult to calculate because neither NTNU nor UCLA measure the volume of resources they spend on formal leadership positions. Contrary to popular belief, self-management is both efficient and effective. It is efficient because little resources have to be spent on formal day-to-day management of work. It is effective in the sense that in general, faculty know what they need to do and plan accordingly, without detailed instructions. Graduate students are evaluated in part on their demonstrated ability to manage their own work with only weekly or monthly guidance. The effectiveness of self-management among faculty was clearly demonstrated when NTNU and UCLA closed due to the Covid-19 pandemic. Around noon on March 12, 2020, NTNU's administration informed students and faculty through a post on the intranet that all normal teaching was canceled. The next day, without further notice faculty moved their teaching online. Face-to-face meetings and seminars were canceled; all interaction between faculty members happened through digital media. This also took place without any commands from top leaders and the administration. The same thing happened at UCLA.

Moreover, both NTNU and UCLA depend on the entrepreneurial efforts of individuals, most obviously with respect to research and mobility of 
knowledge, but also in efforts to establish teaching collaboration across departments and didactical experiments. Entrepreneurship is expected, such as successful acquisition of external funding. The expectation is also articulated through policies for innovation and interdisciplinarity. Both Knut and Sharon have been actively engaged in such work throughout our careers. We return to such policies in Chapter 5. Obviously, the conditions for exercising autonomy are different for those temporarily employed - PhDs, post docs, lecturers, and so on - compared to tenured faculty. There is less risk involved for the latter group when they embark on new initiatives or resist bureaucratic efforts to shape their work. Entrepreneurship is time consuming and may not pay off. Knut spent a considerable part of his post doc period trying to establish a center for technology studies at NTNU, together with a couple of other post docs. We organized an informal center, initiated interdisciplinary seminars, wrote annual reports, held meetings with university officials, applied successfully for research grants - but the administration had other priorities. Then, by a stroke of luck, one of the research councils set up a call for a technology studies center. This call got the attention of the university rector who intervened to provide funding for a small, temporary technology studies center at NTNU where Knut was eventually hired. While still a graduate student, Sharon was asked by a dean to design a science and technology studies program from a humanities perspective. It was a demanding, but very interesting project, although nothing came of it. Later during her job interview at the MIT Science, Technology, and Society Program, she was able to refer to the report, indicating she had suggestions for further expansion of the existing program. The MIT faculty members were surprised that a graduate student had been thinking about 'institution building' for new lines of inquiry.

However, it would be naïve to ignore the fact that the autonomy of faculty at both NTNU and UCLA is under authoritarian pressure from top leadership and administration through their striving for excellence, efficiency, and reputation. We discussed this at length earlier in this chapter by analyzing how academic freedom is being circumscribed and partly eroded through bureaucratizing and corporatizing. When we nevertheless emphasize academic autonomy and self-management, it is because these features remain important, not only for our ability to conduct academic work in a proper way, but also because they are a base of resilience and resistance toward neoliberal efforts of control and restraint. Given the pervasiveness and strength of the neoliberal efforts, we have been struck by the lack of protests against the neoliberal measures and what seems like docile acceptance of authoritarian governance among faculty members. Certainly, there are complaints, but the huge majority remains silent. This may reflect an authoritarian feature of the academic system, where knowledge is considered more important than democracy. 'The university is not a marketplace of ideas in the sense that any opinion is worth hearing' (Scott, 2019: 5). However, our experience is that 
the lack of protests also reflects unwillingness to spend time and resources on such engagement. Rather, faculty members prioritize the activities where they may exercise control and harvest benefits - their research, teaching, meshworking, and so on. Moreover, many faculty members do not seem to consider authoritarian governance that much of a nuisance. Most choose to ignore the invasive epistemic governance of the bureaucratic infrastructures increasingly imposed on everyday academic activities. Some also find it convenient that other people do the work of interacting with policy makers and similar stakeholders, as long as they believe they are still allowed considerable autonomy and self-management.

We also get the impression that most academics consider public articulation of critical opinions about university development as useless. The situation is like what Herbert Marcuse (1964) characterized as repressive tolerance. Faculty members have freedom of speech, but few even listen to critical interventions. Instead of articulating dissatisfaction, many choose to ignore the governance initiatives of the leadership and the nagging about excellence and efficiency. Others more actively choose to remain ignorant about the implications of the specific forms of algorithmic governance being inserted in every university activity. However, some are fascinated by the excellence talk that works as a governmentality measure among very ambitious and competitive academics. Thus, they accept excellence demands because they themselves want to generate the kinds of academic achievements that are supposed to be rewarded. This points to yet another feature of the relativeness of academic freedom - the susceptibility of many academics to governmentality and incentives that discipline us to increase our research and teaching efforts.

\section{Academic Autonomy and the Regime of Meritocracy}

As mentioned above, collegial organizing practices rest on the belief that knowledge plays a decisive role in university decision making. This belief is co-produced with the idea that position and authority in universities are based on merit; that belief sometimes requires the 'willing suspension of disbelief' as Aristotle indicated theater audiences routinely do. This regime of meritocracy shapes collegial organizing in paradoxical ways, due to its ghostlike qualities. Universities' quest for excellence is openly articulated through intersecting discourses of quality, public trust, political expectations, and the role of metrics. Merit is ubiquitous, a self-evident aspect of academic life, but its definition and assessment are contextual and contingent.

The principle that achievements should count rather than various social characteristics (such as class, gender, race, sexuality, nationality, bourgeois comportment, and ethnic background) is a shared ideal, but at the same time it is known frequently to be violated in practice. A century ago Max Weber (2020 [1919]) claimed that 'Academic life, in short, is an utter gamble.' He further warned that a young scholar who wants to pursue an academic career 
needs to be able 'to bear to see mediocrity after mediocrity promoted ahead of him [sic], year after year, without becoming embittered and broken inside' (p. 10).

The paradoxical, continued reproduction of the regime of meritocracy we see as based on a willingness of academics to be assessed on our merits while risking that these merits will not be appreciated by the evaluators. We know that scientific and scholarly knowledge often is contested and that assessments are influenced by intellectual tastes and preferences, but we still prepare for and participate in such assessments. We may do so tongue in cheek, sometimes bending the format, but nevertheless, we contribute. As Weber observed, there is a strong element of luck in academic assessments. However, in our experience, this knowledge tends to be suppressed into a kind of academic sub-consciousness. Many academics are critical of the regime of meritocracy, but their concern is with assessment criteria and practices. In this manner, it seems that we academics need to believe in the regime of meritocracy, and its handmaiden, peer review, even if we know they are contingent and can lead to biased outcomes. We need this belief because it provides a rationale for hard work and a hope of future success. Also, the regime legitimizes academic freedom and autonomy as achieved and productive privileges that help to provide public goods. This is policed through peer review of our professional performances through scientific/scholarly publications, quality of teaching and scientific/scholarly engagement with society. Academic freedom is not a birthright.

Still, when we see that the ideal is sidestepped, for example in processes of hiring and promotion, this produces considerable affect. In an ongoing study of letters of complaint, filed as response to hiring assessments at the faculty of humanities at NTNU, this is very clear. ${ }^{16}$ Most of the complaints endorse meritocratic principles; the problem is that they have been applied improperly. This is sometimes stated in an ironic way, sometimes expressed with fury. In any case, the meritocratic regime is expected to be helpful, career-wise. More generally, universities are expected to uphold and protect this regime as a way of maintaining public and academic respect. Thus, it is striking that the study at NTNU found that such letters of complaint were rare. Probably, most applicants choose to keep their disappointment and fury to themselves, at best sharing this with a few close friends. We are very familiar with these practices of grief, worry, frustration, depression, and anger.

Unsurprisingly, the analysis of the letters of complaint further shows that there are different expectations regarding what should count as an example of merit. Does unpublished work count? What about publications in Norwegian that cannot be read by an international audience? Of considerable importance is the longstanding debate of the relative importance of research and teaching, found at many universities. Traditionally, publications have been decisive in hiring and promotions, but increasingly, teaching experience and resources are given weight. Other activities and achievements, such as 
academic service work, success at getting research grants, and experience with research collaboration, are progressively emphasized in some contexts, but scorned in others. When we are being evaluated by any academic in our larger discipline and not by someone informed by the scholarship in our specific research area, we know there was, in fact, no peer review. While the appreciation of publications involved subjective valuation, the increased scope of merit makes evaluations even less transparent. The study of the complaint letters clearly shows how opacity becomes a problem, making assessments less robust. We have observed that junior scholars often believe that the only merit that counts is publications, preferably in so-called high-ranking journals. They criticize this practice while focusing their academic efforts singularly on publications. In this way, they may become victims of increasingly opaque assessments when they neglect cultivating other attainments than publishing.

Academic freedom and autonomy are discursively embedded in merit, and peer review remains the dominant process of assessment. This makes peer review a cornerstone of collegial practices, but that is not well defined or regulated. For example, at UCLA most departments include only one or two people in each scholarly subfield. Our research colleagues are in different universities, not in the same department. Our department colleagues might be in the same discipline or larger field of inquiry, such as anthropology or physics, but we are likely to know relatively little of the history or current debates in each other's subfields of research. Peer reviews of our work, including how we teach that material, should necessarily be done by our research peers, almost always found elsewhere. The widespread critique, or even careful examination of peer review assessment practices, could weaken collegial systems, but there are additional practices that provide resilience. We have already mentioned the efficiency and effectiveness of self-management, another pillar of collegial organizing principles. This points to the critical importance of the conduct of academic citizenship.

\section{Academic Citizenship as Legitimizing Academic Freedom}

Academic freedom allows for autonomous spaces of decision making with respect to research and teaching. These spaces are not given much concern in present discourses about universities, be they critical or based on neoliberal ideology. However, as we have argued, the spaces are vital to the running of universities and the self-management of faculty members. To uphold them requires conduct of good academic citizenship in the sense that this conduct provides evidence for and trust in our ability of self-management. Bruce Macfarlane (2007) defines academic citizenship as the pursuit of virtues related to performing services to students, colleagues, and society. In this manner, the concept mainly refers to the academic service work that is critical to make universities function, but much less visible and rewarding compared 
to research and teaching. We extend the concept to include normative aspects of the performance of all academic tasks, including research and teaching. To ask about academic citizenship is to raise questions such as: What is good research? What is good teaching? What is appropriate collegial behavior? Thus, the concept covers the trinity of research, teaching, and service. To be a good academic citizen means to engage broadly in these activities to provide public goods of reasonable quality in a virtuous manner.

There are many sources of relevant norms, such as codes of research ethics, discourses such as Robert Merton's characterization of the ethos of science as communism, universalism, disinterestedness, and organized skepticism (often familiarly abbreviated as CUDOS), or the implicit standards of academic community. Most of us have pretty good ideas about what we should do to be good citizens. Thus, it is unsurprising that at NTNU and UCLA we observe that academic citizenship mainly is an object of policing practices instigated to stop obvious misbehavior and that most of the policing is ineffective. Most prominently, there are ethics committees that may address complaints. Students are trained in research ethics, and there are bureaucratic procedures in place to secure ethical research practices, such as mandatory informed consent forms. Both universities have formal procedures to manage complaints about sexual and racial harassment, although the effectiveness of these procedures has proved to be questionable. The policies all focus on the actions of individuals; they do not address institutional racism and sexism, or other forms of institutional inequities, formal or informal. Neither university has bothered to provide a guide to good academic citizenship for individuals, small groups, and the university as a whole. Silence prevails on that topic.

Maybe we could extract relevant guidelines from advertisements for academic positions and hiring practices. The common suspicion is that we would end up by defining good academic citizenship in terms of research excellence, which would preclude important reflections regarding what is good research. However, when we study hiring practices, we see that research excellence is not that predominant. Such focus tends to be moderated by concerns regarding social relevance and the ability of university research to provide public goods that external stakeholders are requesting, even demanding. Increasingly, the criterion of research excellence is replaced by a concern for 'suitability', which reflects a wider concern for academic citizenship.

Present policy discourses about grand societal challenges clearly have an impact. UCLA has started a grand challenges initiative that is presented as connecting 'faculty, students and supporters from all disciplines to work together, adopting a holistic approach to solve critical issues'. ${ }^{17}$ Two challenges have been in focus; to help Los Angeles to become sustainable and to help understanding, preventing, and treating depressive disorders. Both initiatives may be considered as indirect efforts to develop academic citizenship to include concerns about collaboration across and beyond disciplines and problem-solving. We miss more outspoken engagement. NTNU 
is required though the award letter from the Ministry of Education and Research to address the grand social challenges that the government prioritize. As part of its response, NTNU has started four strategic research areas directed at energy, health, oceans, and sustainability. The underlying rationale is similar to UCLA's initiative. 'Research, education and innovation are lifted and intensified through interdisciplinary collaboration. ${ }^{18}$ To what extent the engagement with grand social challenges impacts the exercise of academic citizenship at the two universities is difficult to assess. Clearly, there is a discursive pressure to make faculty members and $\mathrm{PhD}$ students consider the social relevance of their research, including interdisciplinary collaboration. According to our observations, the pressure has effect in the sense that faculty members and PhD students are clearly aware of these expectations. However, many express frustrations about having to balance excellence and relevance and find this balance difficult. Neither NTNU nor UCLA offer any official guidance about how to deal with this.

Concerns with respect to teaching are probably even more important to moderate the demand for research excellence as the main aspect of academic citizenship. The quality of university teaching has received increasing attention, partly in response to the comprehensive changes in the size and composition of the student body. Often, we hear complaints that faculty members prioritize research over teaching, that research has much more prestige than teaching, and consequently that the quality of teaching is not good enough. Thus, critics argue that the university culture is insufficiently appreciative of teaching efforts, and that faculty lacks pedagogical training and tend to keep to teaching methods that are considered old-fashioned and ineffective.

Such engagement changes academic citizenship, putting greater emphasis on both the relevance of research and on teaching quality. An important aspect is the growing emphasis on students' assessments of teaching, which is institutionalized through new regimes of reporting. Gaming the teaching metrics is as developed as gaming the research productivity metrics. Many students have become neoliberal too, increasingly demanding transactional pedagogies: courses with clearly demarcated goals and highly specified requirements for each possible grade which they then want to negotiate. Many want a marketable 'skill set' instead of an education, much less an understanding of how to recognize and assess knowledge making or learn how to make some in new ways.

Increasingly, faculty members must participate in mandatory pedagogical programs. When applying for tenured positions or promotions, applicants must provide comprehensive documentation of teaching activities and achievements. This is not enough. We need broader, public considerations of academic citizenship, in particular to include reflections on academic selfmanagement and the normative regulation of academic communities to make them more emphatic and liberal. This is about cultivating generosity and 
collaborative forms of competition. To defend academic freedom, we need to demonstrate virtuous academic citizenship. As we have seen in this chapter, in this respect we have some way to go.

\section{Epistemic Politics and Collegial Relations}

At the heart of the argument in this chapter is epistemic politics, the local arguments about what constitutes proper academic expertise, the range of that expertise, and who may be considered experts (Doing, 2004). This concerns both research and teaching. In principle, academic freedom guarantees that academic peers have an exclusive right to participate in epistemic politics, but substantial boundary work is conducted to regulate and police such participation. Factions feed on such boundary work, which is legitimized by metric measures of meritocratic practices. Bureaucratizing and corporatizing efforts circumscribe epistemic politics; in particular bureaucratic measures may introduce formalized practices regarding reports and assessments that preclude informed discussions of quality. The allocation of resources may empower or disempower factions and thus change some of the premises of epistemic politics. Nevertheless, epistemic politics have remained a collegial affair. Without relevant academic accreditations, participation is at best difficult.

Neoliberal policies have aimed to confine epistemic politics and reduce its importance because epistemic politics hinder the transformation of universities into a marketplace of ideas where economic valuations dominate. Quests for excellence also impose on epistemic politics, since the metrics involved in such quests represent additional criteria in the assessment of expertise. Excellence is an exterior and not an intrinsic quality of knowledge. The quest metrics impose a system of valuation of expertise where it is more important how findings are published than their epistemic quality.

We have proposed academic citizenship as a norm that should reshape epistemic politics to make it more virtuous. Some have criticized that the concept of virtue, built with the Latin words for 'man' and 'merit', is necessarily saturated with the performance of entitlement enabled by certain kinds of class and gender privilege, or worse yet, only those with such privileges are capable of virtue. We mean it in a rather straightforward way: the struggle to understand, act, and work with others 'in the direction of goodness' toward all. We have shown how the conduct of epistemic politics may cause discomfort and marginalization of academics. Proper academic citizenship should help moderate the power gaming sometimes present in epistemic politics, thus making academic life fairer and more inclusive. Another way of conceiving academic citizenship is as the outcome of academic subject formation. This is the focus of the next chapter where we explore processes of becoming an academic in the time of entrepreneurial universities, including research training and performance assessment. The formation of academic subjects as 
entrepreneurial is of course shaped by the political economies of our universities, but as we shall see, also by the academic culture.

\section{Conclusion}

We have examined different governance practices, ranging from academic capitalism, algorithmic governance, academic freedom, corporatism, peer review, entrepreneurial individualism, regulatory administration, collaborative 'start-up' ventures, cronyism, bureaucratism, and so on. What does it mean for universities that all those kinds of governance are being employed at the same place while there is so little interaction or disputation among them? Why all the indifference and silence, aside for the occasional stylized encounters, such as at ceremonial events or protests?

Sharon is reminded of the history of California, first occupied by a very large array of indigenous communities, mostly occupying different ecological niches. Then a long series of occupations began by waves of colonialists: Spaniards, Portuguese, Mexicans, Russians, and Americans, among others, some arriving simultaneously. Most of those groups have been as diverse as the first indigenous occupants, with many different kinds of plans for occupation, some pursued, some discarded. Each wave brought new political economies with different kinds of rankings, exclusions, and violence; almost all have left their infrastructures, resources, and toxic waste. Like Rome, the ruins of each layer, along with their accomplishments and wealth, elites and oppressed, have been either incorporated or ignored in building later empires. For example, if we want to understand contemporary water politics in California, such as the domain of the City of Los Angeles Department of Water and Power, we must understand that all of that past is still very much present, embedded in the layers of epistemic and material infrastructures that determine how water gets from one place to another, or not, along with all the toll charges at every interface.

UCLA is like California. It has been occupied by waves of ambitions, plans, reforms, exploitations, and achievements, each new one adding to the complexity of what it found. Lineages of each kind occupy the university today, along with the more recent arrivals, all with their own ways of ignoring the others, perhaps with a twist of contempt. Some have comfortably taken control of some niche, building a fiefdom within it. Some try to expand their domains, creating vassals among others who must provide specific services in exchange for certain benefits of territory, offices, rank, and so on, including the power to simply be ignored. At UCLA we are awash in academic capitalists, corporate algorithmists, bureaucrats, regulators, reviewers, entrepreneurs, collaborators, and cronies, some old timers, and some newcomers. For the last several decades a new configuration has taken hold, with the capitalists and algorithmists in an odd marriage to control the 'senior professional' level of the administration which has multiplied stunningly since 1975 , relative to the 
number of faculty and lower staff, as noted elsewhere in this book. In turn, that odd couple has made accommodation arrangements with many of the others, most of whom prefer to not acknowledge they have done so. Without discussion or protest by those with influence, the corporate algorithms and capitalist priorities are practiced everywhere, even by those we might expect to be protesting vehemently. Metrics of diversity and productivity are widely discussed, but power and quality are rarely mentioned.

Are capitalism and corporate algorithmics altered in academia nearly beyond recognition? Of course, but their origins remain obvious. Have bureaucrats and regulators managed to maintain control of their classifications and exclusions? Definitely not, but the regulatory apparatus is everywhere. Have faculty autonomy, peer review, and academic freedom changed fundamentally? Certainly, although they have survived in new forms, with most parts redefined by regulations, algorithms, and academic capitalism. Has there been a substantial increase in sycophantic cronies and ruthless bullies? Yes. The widely practiced decorum of ignoring the existence of the others in the crowded academic arena leaves the bullies to manage the traffic among all the different groups. Are there limits on the designs of each of the groups? Very few are found at UCLA where it seems we work in a space of unlimited speculation with fortunes frequently made and lost. The only external authorities are the auditors from the State of California and the financial regulators from national governmental agencies. Civil society does matter, as do the 'revenue streams' from student fees, donors, research funders, and event fees. Usually, those relationships are managed effectively by university branding with its stellar rankings, even if disrupted momentarily by the global pandemic. From a distance NTNU seems far less turbulent. In these matters scale is probably significant: the scale of the resources to be accumulated and allocated locally. At NTNU, the Norwegian government gathers the resources and influences much of their distribution on campus. That is decidedly not the case at UCLA.

\section{Notes}

1 UCLA's Common Data Set 2010-2020 is found here: https://apb.ucla.edu/cam pus-statistics/common-data-set. See too the Common Data Set Initiative https:// commondataset.org/ and UCLA Office of Academic Planning and Budget (APB) https://apb.ucla.edu/, which includes the Chancellor's Office of Data Analytics (CODA) https://apb.ucla.edu/campus-statistics/overview as well as UCLA Office of Research and Creative Activities www3.research.ucla.edu/about/stats

2 https://en.wikipedia.org/wiki/Learning_management_system

3 https://en.wikipedia.org/wiki/Collaborative_learning

4 On Moodle see https://moodle.org/. The quotation is from a 2007 interview conducted by Ken Udas with Ruth Sabean, then Director of Educational Technology in the UCLA Office of Information Technology. https://vendor.cnx. org/contents/91Itzn4r@7.2:R99BDs4o@8/Ruth-Sabean-Interview-Part-2-UCLASelects-Open-Source-Solution 


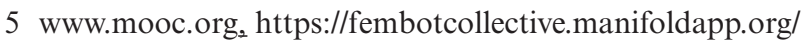

6 https://en.wikipedia.org/wiki/Instructure

7 Lu, T. J. (2021). Employee Data Tables in the University of California (19642015) https://doi.org/10.17605/OSF.IO/BCZQ4 (accessed June 23, 2021) and Lu, T.J. (2015), 'UCLA Senior Management Growth in Comparison to Faculty and Students (1993-2014)'. https://scaleatucla.weebly.com/ucla-senior-managementgrowth-in-comparison-to-faculty-and-students-1993-2014.html (accessed June 23, 2021).

8 The UCLA Faculty Association Newsletter, Fall 2006, reported that 'In Oct. 2005, at UCLA, Letters \& Sciences, 97\% of Assistant Professors were Off Scale; 94\% of Associates; and $82 \%$ of Full Professors were Off Scale' https://uclafa.org/newslett ers/uc-shadow-salary-scale/. An October 7. 2020 report from the UC Task Force on Faculty Salary Scales recommended that UC 'progressively decrease off-scale increments'. They added that 'The current model is not transparent. There is no clear policy for annual adjustments to the salary scales that reflect market rates and that can be relied on when discussing faculty compensation with the Regents and the State. The current model relies heavily on off-scale compensation to cover the gap between on-scale salary and market rates. Such additional compensation likewise lacks transparency because it the off-scale amount is discretionary and typically determined by an administrator, such as a dean. In 1999, 50\% of General Campus ladder-rank and equivalent (LRE) faculty were paid off-scale, and 6\% of total faculty pay was off-scale. In 2019, $99 \%$ of General Campus LRE faculty were paid off-scale, and $24 \%$ of total faculty pay was off-scale.' They go on to declare that 'Off-scale compensation is pernicious. Off-scale compensation creates multiple problems.' https://senate.universityofcalifornia.edu/_files/reports/mg-mbfaculty-salary-scales-task-force-report.pdf

For historic and current UC Academic Salary Scales see www.ucop.edu/ academic-personnel-programs/compensation/index.html and www.ucop.edu/ academic-personnel-programs/compensation/2020-21-academic-salary-scales. html. For 2019 salaries of each UC employee see the Sacramento Bee State Worker Salary Database at www.sacbee.com/news/databases/state-pay/article229468549. html

9 'Angela Davis returns to UCLA classroom 45 years after controversy. The Regents' Lecturer will take the stage at Royce Hall again.' UCLA Newsroom, May 5, 2014. https://newsroom.ucla.edu/stories/angela-davis-returns-to-uclaclassroom-45-years-after-controversy

10 www.regjeringen.no/no/dokumenter/prop.-111-1-20202021/id2840742/

11 Ibid., p. 12.

12 www.ucla.edu/search?qa =academic $\% 20$ freedom

13 https://innsida.ntnu.no/wiki/-/wiki/Norsk/BEVISST+-+System+for+virksomhets styring (accessed April 30, 2021.

14 UCLA FAQ on Hub and Spoke: https://evcp.ucla.edu/priorities/hub-and-spoke-faq/

15 www.senate.ucla.edu/bylaws/appendices/app16

16 The study was undertaken by Sofia Moratti and Knut H. Sørensen and is in the process of being published.

17 https://grandchallenges.ucla.edu/about/

18 www.ntnu.edu/research/strategicareas 


\section{Subject Formation and \\ Re-formation Throughout \\ Academic Careers}

\section{The Double Bind of Disciplined Entrepreneurs}

\section{Introduction}

In this book we have been discussing entrepreneurial universities, those questing higher and higher national and international rankings, along with greater 'revenue streams from extramural research funding, private donor contributions, student fees, and campus event fees. As that entrepreneurial ecology has emerged in US universities since the 1970s, and in a more moderate fashion in Norway after 1990, the various participants have changed too, also in response to the need to navigate internal resources and politics. In this chapter we address how university faculty members and students learn to become what they should want to be, their formation as academic subjects that must strategize for and within entrepreneurial universities in their quests for becoming excellent in these turbulent new ecologies. We also address how their decisions and choices are situated in universities that are corporatizing and bureaucratizing in strategic ways that rely upon new kinds of entrepreneurial subjectivities among their members. We ask too how those increasingly entrepreneurial subjects might be altering universities.

People have been becoming academics for centuries. Some of the early practices persist, as do those from successive waves of change. Those archaeologies of pedagogies are not experienced as layers of the past, but as a complex, conflicting morass of urgent requirements and everyday routines. Similarly, established practitioners in each field, old or new, might regard without reflection that their own formation was simply normal and see no reason to not reproduce it. Because Knut and Sharon have participated in several disciplines and many interstitial academic arenas, our own experience with the changing practices of academic subject formation are far wider than the norm. Most of our colleagues have experienced one model and many presume that is what their students are doing. Here we report on that set of differing expectations and practices now found on our campuses and our own earlier experiences as the current changes in universities were still new.

The processes of becoming an entrepreneurial academic might well last throughout whole careers, as they have ours. This means that we may face 
forces that try to re-form us as academics, and our ways of coping with such forces may change. In the previous chapters, we have repeatedly criticized the idea of a globally uniform neoliberal university and juxtaposing our observations from NTNU and UCLA to demonstrate some of the existing diversity. We continue this effort when we study the formation of academic subjects, recognizing that we are based in distinctly different academic systems. We explored features of the local political economies in Chapter 2 and cultural diversities in Chapter 3. Here, we juxtapose our observations of different regimes of subject formations. In addition to clear differences in the organization of research training and the working conditions of young academics, US universities follow a tenure-track system with continued individual assessments, while Norwegian universities offer permanent employment once hired into a faculty position. We explore some consequences of this variation.

In doing so, we return to the topics of meritocracy and academic citizenship. Meritocracy may be seen to provide an ideological foundation of academic subject formation; its double-bind quality can both motivate and inflict pain (Bateson, 1972/2000). Academic citizenship is acquired; it is a set of practices, civilities, ethics, comportment, and virtues that shapes the way we may influence the becoming processes of others. Good academic citizens are helpful, but academics may also learn to inflict harm upon others as might have been inflicted on us. There are multiple subtexts to all this strategic thinking about changing ecologies and the growing demand for entrepreneurial academics for entrepreneurial universities. Among those are discussions about how to either exploit all this for personal power or share our strategies for mutual aid. Differentiating those two and yet others is sometimes not easy. For example, knowing someone's position in global and national politics is not necessarily a guide to whether or not they support doxa, canons, and authoritarian hierarchies within the academy and vice versa. Campus and epistemic politics often seem independent of politics at large.

To begin, we focus on the decision to become an academic: what kind of advanced degree to seek and what kind of knowledge making to pursue. We then examine the situation of graduate students pursuing doctoral degrees $(\mathrm{PhDs})$. The idea of the $\mathrm{PhD}$ as an advanced degree and a certificate of a high level of research qualifications emerged with the research university in Germany (Clark 2008). However, thinking about the $\mathrm{PhD}$ as the outcome of a long period of organized training came later and was in particular shaped by the practices of US universities from the 1860s. Graduate schools where $\mathrm{PhD}$ training is separated from undergraduate and subsequent professional education is a US innovation. Organized training of early career academics came later in other countries. In Norway, such initiatives first emerged in the 1980s, mainly as programs based in departments or faculties. Overall, there are considerable international variations with respect to what is involved in becoming a PhD and becoming an academic (McAlpine and Åkerlind, 2010). 
In both Norway and the US, courses of study and degree programs, from the bachelor's to the doctorate, are conducted within departments. Over the last 25 years a large administrative apparatus has emerged in the US to manage a growing regime of rules and reports about the graduate students, campuswide; what had been a rather small office at UCLA now has dozens of staff members and is led by a vice chancellor. Every department also has a staff member assigned to maintain liaison with the graduate division office and to keep the students informed about all the required reports and procedures. There are no such 'graduate divisions' in Norway.

The development of $\mathrm{PhD}$ programs has been an important driver behind the vast expansion of university research in absolute, but also relative terms, compared to teaching. They are a major component of international university rankings, although undergraduate programs also can influence commercial rankings within countries. Moreover, graduate students are a very substantial resource for the research efforts of universities, as well as an important element in the changes toward collaborative ways of organizing research. Arguably, $\mathrm{PhD}$ programs are machineries of research as well as research training. Relatively speaking, graduate students are also cheap labor. New forms of graduate and professional education also constitute important new revenue streams for universities.

Getting a $\mathrm{PhD}$ is not the end point of becoming an academic; at best it is the end of the beginning. We discuss some of the possibilities and challenges academics meet further on in their careers, with an emphasis on cultures of assessments while notions of quality and merit are under constant revision. Academics are continuously assessed, in informal collegial settings as well on more formal occasions. We show not only the diversity of such cultures, but also discuss how they may be experienced. Academic life is filled with corridor talk and implicit, tacit knowledge about how systems may be navigated. We want to explicate this. Some might argue like Showalter (2005) that a large trove of novels about academia show that the pain induced by academic power plays and competitiveness has been part of universities since the 19 th century, at least. Of course, academics have been taught and bullied for centuries into wanting to be the kind of academics we should be, within the old, but persistent requirements of ritual subordination within complex hierarchies and the frequent, stylized competition for the same scarce resources on campus. Indeed, the structure of the lengthy contemporary apprenticeship in academia (up to 15 years in the US) has many parallels with that of the medieval craft guilds (Traweek 2006). However, we argue that there are now new demands laced into the old. Our focus is on how the current developments in universities have added something new to those conventional practices, beyond 'publish or perish', as we are expected to be entrepreneurs in some new 'start-up' initiative, while also being a good corporate employee, assisting in meeting yet another new 'goal' defined elsewhere, and working 
with various extramural 'stakeholders' and communities, plus 'building the brand' and improving the rankings.

\section{Entrepreneurship in Academia}

We have argued that UCLA has been more focused on building a corporate university model, while NTNU is more engaged in bureaucratizing, keeping in mind that these projects are not mutually exclusive. We might expect that the ways of becoming the right kind of academic in those two settings might be different, and they are. However, there also are some similarities and there are contradictions within each. How does UCLA make academics fit for a corporate life and yet require entrepreneurship? In fact, management schools have been addressing the paradox of corporate entrepreneurship for 40 years: specifying definitions, typologies, implementations, assessments, refinements, and pedagogies, all while invoking it as a fundamental characteristic of global information-based political economies (Burgelman, 1983; Covin and Miles, 1999; Kuratko and Morris, 2018; Phan et al., 2009; Schendel, 1990; Wolcott and Lippitz, 2007). Universities are far from original in demanding this new kind of worker, nor are academics the only ones to find the persona problematic. Presently, there also is much concern about academic precarity and how this may be addressed (OECD, 2021). In some putative golden age, most of those who embarked on the path to becoming an academic did so. Now there are a series of 'purgatories' in which supplicants can petition for advancement at one stage or another ad nauseum, with many failing to succeed. The survivors can feel simultaneously relieved, unworthy, and entitled. We consider academics' need to cope with precarity as an additional effect of entrepreneurial universities and an issue of academic subject formation, to which we return at the end of the chapter.

The old ideal of having a 'calling' to practice a profession, in this case knowledge making, has been supplanted during the last 50 years by a demand for metric productivity, which we discussed in Chapter 2. This is best met by staying strictly within the same topic of inquiry, making a large 'start-up' time investment only once, and then using that knowledge to generate as many small contributions as rapidly and as long as possible. Within this frame of thinking, teaching should be done transactionally, specifying as much as possible in an easily reproduced syllabus, read by students as a contract. We can all become entrepreneurial in the sense of 'gaming the system' to generate the best metrics. All works smoothly, as long as we want to do as we should, formed as appropriate academic subjects. How is this achieved, and at what costs and benefits to whom and what? There is another definition of entrepreneurialism: the demand for innovation, originality and transdisciplinary work, which become far more elusive in the context of maximizing productivity metrics (Felt et al., 2013). Being that kind of entrepreneurial academic 
might be incompatible with the others while still in limited demand within some kinds of universities.

When we discuss the becoming of an academic framed through the mixed metaphor of entrepreneurship this is not just to reflect on some consequences of universities becoming entrepreneurial in the sense of broadening their sources of income (Clark, 2004). We see this concept as having several dimensions. One focuses on the ability to deal with extramural actors and interests, including the acquisition of the best applicants, donations, and research grants. Another emphasizes the resources involved in navigating the academic system as a hierarchy, but also as an ecology of knowledge. Moreover, the focus on entrepreneurship also means attention to agency and the process of becoming an academic actor in the context of striving for 'excellence'. Yet another paradox emerges with this corporate entrepreneurship; who or what is the actor? Is it the 'incorporated' university, the department, a field of inquiry, a research team, or the individual scholar? The prevailing epistemic politics require that the proper entrepreneurial actor be an individual working within a discipline, yet the corporate, bureaucratizing university is an assemblage of many forces, strategies, divisions of labor, missions, and personnel. What is the entrepreneurial choreography for that amorphous entity?

\section{Subject Formation in Academia}

Many scholars, such as Felt and Fochler (2012), Fleming (2021), Gill (2009; 2014), and Pereira (2017) have explored the harsh costs of becoming the right kind of academic in today's entrepreneurial universities in Australia, Austria, and the UK. However, we are well aware that many academics regard such matters as private and personal, with no place in the scholarly literature, and to do so is inappropriately 'confessional' or 'subjective'. Instead, we regard the contemporary forms of 'subject formation' to be part of the process of generating the right kind of person to work effectively in our changing universities. This approach has a long history, far too long to rehearse here. From studies of worldviews and climates of opinion to thought collectives, mentalities, paradigms, and epistemes, and then structures of feeling, ways of seeing, and habitus scholars have addressed how our embodied engagement with ideas, affect, action, and each other in specific historical political economies are co-constituted and continuously co-morph. To focus on ideas without attending to affect and action are strangely partial accounts. We require 'strong objectivity', accounting for the changing standpoints and positionalities in the making of our 'situated knowledges' (Collins, 1997; Haraway, 1988; Harding, 2009).

Fanon (1963) showed how we incorporate not only the judgments of powerful others about us, but their justification, as well. In academia we learn to believe in the adjudication system, however harsh it might be, before we 
learn our place in it, so we usually accept others' pronouncements about the quality of our own work. Deleuze and Guattari (1983) discussed how political economies must have the just right kind of actors, such as a certain kind of consuming subject, always desiring the next new product or experience, while quickly bored by the ones most recently consumed. In today's academia most want to be seen as framing the newest ideas, not advocating older ones. There is a strong commitment to teleology, believing that the most recent must be the best. Foucault (1977) examined how we are 'disciplined and punished' into becoming an effective actor, how our subjectivity is shaped by the ways we are classified and engage with the norms around us, even when harshly confined. In Japan our academic disciplines are called takobako, designed for catching an octopus that can swim into the box easily, but then cannot get out.

As both participants and observers, our work invokes and challenges this long, active scholarly inquiry about the shaping of affect and consciousness as a necessary part of creating contemporary academic subjects. This chapter includes Knut's and Sharon's autoethnographic comments on some of our own subject formation experiences in academia. In the first chapter we discussed autoethnography in greater detail. Succinctly, in this chapter we ask about the everyday cultural messages in universities guiding cohorts through our career-long, successive apprenticeships. What are the options among the expectations and how do the academics at that stage learn how they are doing? How can they succeed and if not, how do they compensate? They might be more or less predisposed to cooperate or at least accommodate themselves to the prevailing expectations. To what extent can those instructions be modified and how? We discuss those practices in the context of powerfully changing ecologies. As UCLA corporatizes and NTNU bureaucratizes, what are the changing expectations for knowing subjects and how do those subjects respond? How do their strategic responses change NTNU and UCLA?

As we have noted, at both UCLA and NTNU academics are increasingly expected to be entrepreneurial. Sharon notes that many of the UCLA undergraduates already are making strategic choices about their educations. As they have decided to become academics, how have their strategic approaches to their education begun to change the conventional university procedures for getting Master's and Doctoral degrees? At UCLA we see such students making epistemic choices that alter many conventional distinctions between kinds of knowledge and ways of making knowledge, distinctions that might have enabled the academic subject formation of their teachers. These days when undergraduates contemplate becoming an academic, they face a very wide array of options among established and new fields of inquiry and among multiple ways of making knowledge. They can assemble a set of degrees from different programs. The entire apparatus is in flux. We describe the options at UCLA, including how the university organizes graduate education and how those changing arrangements fit into its corporatizing strategies. Next, we 
describe the rather conventional features of getting a doctoral degree and how students work to modify it to fit their goals.

Then we discuss the assessments that punctuate academic careers. At each career stage academics must show we understand the current expectations of us: eventually we need to do work that gains recognition from influential colleagues internationally; we need access to research funding from important extramural sources, to publishing our work in the appropriate venues, and, of course, to find employment in academia. Mentors can facilitate all that, but the candidate must be seen as pursuing those goals as an individual, competitive entrepreneur. Only after those criteria have been met, graduate students should get doctoral degrees and find faculty positions, then become successful researchers, well evaluated teachers, and participate in university governance productively, while very much wanting to do so. We all falter at some stages and learn from our errors; others watch and learn, too. Sharon and Knut describe their own experiences with the normative expectations in contemporary academia.

\section{Becoming Academics in the US: Disciplined and Entrepreneurial Epistemic Choices - Sharon's Account}

In the United States the path to becoming an academic begins with the decision to seek education beyond the Bachelor of Arts (BA) or a Bachelor of Science (BS) degree, received at the end of undergraduate studies, which typically lasts four years with a concentration the last two years in one field, referred to as a 'major'. Increasingly, the more entrepreneurial students study other fields as well and meet the requirements for completing a second major, or a minor. Those students see this as a strategy to increase future opportunities. For example, in order to create more impressive applications, ambitious students planning to attend medical school might take many courses in biology, but major and minor in other fields, such as disability or gender studies; similarly, a student planning a career in law might take many courses in political science and history, while majoring in computer science. To simply major in biology or 'pre-law' is seen as particularly unimaginative; to do more and do all of it well is the strategy. US universities oblige by providing extensive guides to curricular requirements for the various assortments of 'double majors' or combinations of majors and minors, while planning for large enrollments in certain classes by non-majors, such as those biology courses required for admission to medical school. Over the last few decades entrepreneurial students have decided that it is strategic to be interdisciplinary; universities and departments have accommodated that practice by redefining majors and minors. An important stress point is that most faculty members did not pursue such interdisciplinary strategies themselves and many are adamantly opposed. 
At UCLA about half of my undergraduate students have said they want an advanced degree (a Master's or Doctoral degree). They must decide what kind of study to pursue and whether to attend graduate or professional school, an important, but rapidly changing distinction in the US. Many students only learn during their final year as an undergraduate about the differences between the two paths and in which arena their own interests might be located. Until about 50 years ago a student who wanted a career as an academic normally would have selected a graduate school, and then become a physicist, sociologist, philosopher, and so on. A student who wanted to become a physician, an attorney, a filmmaker, an architect, a school principal, and so on, would have chosen a professional school. Now students might want various combinations as part of a strategic plan. There also has been a vast expansion in university-based professional schools over the last 50 years: the number of programs, students, faculty members, and funding, all of which represent significant income for universities, far more than the graduate school programs. In turn, the professional schools have assumed much greater influence within universities as a whole. The expansion of choices for advanced degrees is part of our report on how universities have been changing. These expanded curricular pathways with new certifications of undergraduate 'double majors' and 'minors' along with new kinds of Master's degrees also represent new ways for universities to expand revenue, along with new pathways to becoming an academic, specializing in certain forms of knowledge making. However, many faculty members oppose these options and try to enforce a more disciplined path, routinely claiming quite incorrectly that there is no success without disciplinarity. Sharon used to ask colleagues uttering that mantra for their evidence, always receiving only a blank stare. Is their ignorance deliberate?

There once was a stark division of labor between doctoral programs in graduate schools lodged in the traditional sectors of universities and the Master's degree programs in the professional school, conventionally at the status periphery of universities. It was also a division of epistemic work. There are multiple classifications of research; Sharon has encountered five kinds among colleagues in anthropology, astronomy, gender studies, history, physics, and STS (science, technology, and society studies) at various universities in Japan, Sweden, and the US. These include:

- Basic research on questions about fundamental processes (the normal domain of graduate schools).

- Research to identify possible applications of basic research findings to existing problems (for example, in biomedical fields this is now often called translational medicine).

- Developing applied research findings into products or services and evaluating their effectiveness (sometimes called 'R\&D' for research and development). 
- Research designed to inform policy makers' so-called 'evidence-based' decision making.

- Data-intensive research explores large data sets for patterns.

The first kind is sometimes called 'curiosity-driven' research, to highlight that it often explores ideas raised by other ideas and it presumably is unmotivated by solving current human problems. Applied research usually tries to answer an odd question: how could we make use of some bit of new knowledge? Both the first and second kinds of research have been conducted in universities for a couple centuries. Traditionally, the third and fourth kinds of research had been done primarily in corporate and government laboratories or government agencies; now those groups often provide funding for such research to be conducted in professional schools at universities, as a kind of 'outsourced' research. Data-intensive research appears to have emerged simultaneously in academia, industry, and government. Conventionally those five kinds of research have employed different epistemic practices for identifying topics, raising questions, posing hypotheses, invoking research methods, and devising interpretive strategies. In turn, each of those epistemic practices had a different status relative to each other and usually were taught in different ecologies with different pedagogies. As universities have become more entrepreneurial, corporate, and bureaucratic while seeking new kinds of extramural engagements, these epistemologies and their pedagogies have been reshaped too.

The very differentiation of kinds of research has become a significant act of 'branding' in some fields of inquiry and practice where they then claim to be the leading practitioners of a certain kind. Meanwhile, others have begun to challenge the epistemic privilege of basic research and begun to assert, sometimes emphatically, that they are not applying knowledge made elsewhere, but that they work at a new interface located at the intersection of basic understanding, experimentation, societal needs, and market creation. Some claim that they have created new ways of knowing, increasingly called 'the fourth paradigm' while invoking the new field of 'data science' (Hey, Tansley, and Tolle, 2009).

The students who want to become academics are now expected to decide by the end of their undergraduate education which of these kinds of knowledgemaking practices they want to pursue. Fifty years ago, the array was far more limited and there was a rough consensus about the status hierarchy among the choices. Going to graduate school and practicing basic research had far more prestige and resources; that is decidedly not the case now. Graduate students often want to link with multiple departments across the entire campus; they want to learn how to conduct several kinds of research on the same topic. Most faculty members are far less interested in or experienced with such epistemological adventures. The point is that the massive expansion of the professional schools, along with their multiple ways of making 
knowledge, plus their engagements with knowledge makers in many other sectors, have been redefining the nature of academia and the practice of being an academic for several decades. At UCLA that process is manifest. Many of the undergraduates and graduate students regard that array as normal and find the formulaic distinctions between 'the college' version of graduate school and that found in the professional schools to be arcane. They are first confronted with the distinctions when they apply for admission to programs for advanced degrees.

\section{Strategic Choices: Place and Pace}

Conventionally, students went to graduate school to study a specific topic within a particular discipline and to acquire some expertise in that research process with the guidance of someone highly regarded in that field. The expectation was that we could spend a lifetime learning everything about that topic. We might regard such work highly and it might have enabled our own; however, US academia has not rewarded such commitments for at least a generation. In the US we can still acquire the knowledge, credentials, support, and network to build a career in academia, but the way to do that has changed drastically over the last 50 years. What are the current practices for acquiring some specialized knowledge and then making use of it as a faculty member?

As already mentioned in other contexts, faculty jobs in the US for new doctorate holders in the humanities and humanistically oriented social sciences have been declining for 50 years. Typically, 100-300 people from around the world apply for every position in every field in the top 500 universities in the US. Increasingly, US students know that in order to have a strong application for one of those scarce positions it is necessary to think strategically from an early stage in graduate school. Many of them have been thinking that way since they were in secondary school. That strategic, entrepreneurial thinking has become central to academia. Just after I arrived at UCLA in the mid1990s, a very senior, prominent academic at another campus of the University of California advised me: now we must all think of ourselves as self-employed. Do not expect the university to define your job or provide you with resources to do it; you must define your goals and assemble the resources to achieve them. Around the same time the university was beginning to evaluate faculty by an ever more elaborate set of 'metrics' about our accumulated resources (including extramural funding and awards) and products (such as publications and graduate students), which was discussed in Chapter 2.

In many other countries most people spend their entire careers, beginning as undergraduates, at one university. However, in the US this practice is rare, at least at the higher ranked schools. It usually is seen as a sign of weakness, both for the student and those doing the faculty recruitment and graduate student admissions. That is, departments that admit their own university's undergraduate students to their graduate program often are seen as too 
weak to attract students educated by others; similarly, students who stay at the same university are seen as having learned only one approach and hence are not intellectually flexible or sophisticated. There is one respected way to affiliate with a department or program in which we studied earlier: go elsewhere and build a strong reputation. We learn to see home in new ways and those at home learn to see us in new ways, too. The widespread slogan is 'go away to come back'. During the last 50 years in the US we learn early that to succeed we should move, circulate, and ideally 'spiral up' to employment at universities of increasingly higher status. Those who stay in the same place can build local domains but rarely build beyond that. Conventional success requires migration. I had received all those messages as a graduate student at a University of California campus in the late 1970s, realizing that if I wanted to return, I had to leave; I was gone 1982-1994. I did have classmates who stayed in California, but almost all accepted lower ranked positions with less opportunities for research. Several took jobs outside academia so that they could continue living in the same place, remaining active in the same communities. In US academia it is the circulating migrant workers who have more access to the better jobs. I find that most undergraduates know nothing of these norms and often think they would have a better chance being admitted to a graduate program or professional school at the same university they have been attending.

Once a student chooses to attend graduate school there are two more paths to consider. Universities that do not grant doctorates (PhDs), might offer Master's degrees in traditional fields with the expectation that their students would then apply to transfer to a doctoral program at another university. Some undergraduate students who think their applications to an important doctoral program might not be strong, decide to enroll in such MA programs, planning to then apply as a beginning graduate student in a more prominent program, lengthening their path to a doctoral degree by one to three years. A significant proportion of the applications to my current department are from such students. Undergraduate colleges are increasingly interested in launching such MA programs for their schools. They provide income in the form of tuition, a workforce of teaching and research assistants for the regular faculty members who are increasingly expected to seek extramural research funding, and they also add to the prestige of the faculty members who then can teach graduate seminars. Many of the faculty hope to publish effectively and gain positions at kinds of universities where they might have gotten their doctorates.

As described above, most faculty members in US graduate schools conventionally conduct so-called 'curiosity-driven', basic research on questions about fundamental processes and teach their doctoral program students to do the same, rather than applied, development, or policy-driven research, much less how to work conduct data-intensive research, all of which are still customarily found in the professional schools. Such graduate students 
usually spend two to three years in seminars, learning about current research and debates. Then they design and conduct an original research project that advances basic, fundamental knowledge in their field of inquiry. The normal time to degree is five to ten years, depending on the field of specialization. (The period is much shorter when students serve as assistants in faculty-led projects and longer when the student must conduct independent research.) In the US about one third of doctoral recipients take faculty positions in colleges and universities. The rest take positions in government, industry, and NGOs, often leading research programs. That is, about two thirds of the students getting doctoral degrees will take their knowledge of how to conduct original research into arenas outside academia, usually while maintaining strong ties with their friends and colleagues in academia. They are embodied examples of 'knowledge transfer'.

Typically, the average normative-time-to-degree (NTD) is much shorter in some departments than others. In some STEM fields students normally do research as assistants on faculty members' projects, whose extramural funding supports the graduate students, including their tuition fees. In other fields students must conduct fully independent research projects. Unless they have independent incomes, such students are supported primarily by working as teaching assistants 50 percent of the time, work that is accompanied by tuition waivers. A low NTD average for a department is highly regarded. It indirectly signals that such departments are 'self-supporting' while those with longer NTDs are using more university resources in the form of TA salaries and tuition waivers. About 20 years ago US universities began to limit the number of years a graduate student could be employed by the university as a teaching assistant and began to reduce the amount of time a student could be enrolled before receiving a degree. Many students in my current department find themselves held to these increasing expectations, but feel they were not fully informed about them at the time of application and enrollment.

\section{Applying to Graduate Schools in the US: Strategically Selecting Reputations}

I recommend that students look carefully at the requirements for the first two years within each program where they are applying, plus the interests of the program's current students, current faculty members, and their affiliated faculty, as well as the placement of their graduate students. I advise them to learn what research funding the faculty members have, where they are publishing, and where they are giving presentations about their work. They should be wary of people still teaching and writing about the same research questions they first addressed 20 years earlier, as in 2000. As mentioned earlier, pressure for quantitatively measured entrepreneurial productivity means it is more efficient to not modify our research topics, queries, interpretive approaches, modes of inquiry, and bibliographies/databases, but mine those accumulated 
resources to manufacture as many research reports as possible. However, to be innovative, students need to learn not only the current issues and techniques being widely explored in their subfield, but also how to generate new ones, another side of entrepreneurship.

The significance and prestige of topics, queries, methods, and interpretive strategies have been rising and falling at faster rates during the last 50 years. To engage in that process effectively students need to learn the history of ideas in their field, including the specifics of why and how they had changed; such knowledge is foundational for learning how to make the next changes, not merely to learn the catechisms of the moment, however arresting, and the mythic teleologies of how it came to be so much better than all that preceded it. Increasingly students realize they must learn how to 'innovate' rather than to become an expert with a specific 'skill set' associated with certain theories and exemplars awaiting further applications. Expertise is associated with an industrial political economy; continuous innovative knowledge making is now part of the information-based political economy.

Unlike universities in most other countries, in the US the reputation of departments, as well as their subfields, have been fluctuating drastically over the last five or six decades. A prominent university might have several weak departments and vice versa. A university prominent 1950-1975 might be much less so now, even if it has maintained significant social prestige; schools seen as peripheral in 1975 might have become internationally powerful by 2000. (We discussed university rankings in Chapter 2.) I warn applicants to beware of departments relying on reputations built upon work done 1530 years earlier in the context of so much change. Such groups attract many students, but those students do not, in turn, circulate far.

Outside the US the research funding, publishing, and academic employment infrastructure is often more stable with the same universities and departments getting the same proportion of national resources they got 25, 50, or 100 years earlier. In the US that practice began to erode in the post-WWII period and that erosion has accelerated since the 1970s. As we discussed earlier, there are many national and international rankings of universities; many of them include rankings of specific departments and programs within those universities because the rank of the whole university is not an indicator of the status of any one department. Those rankings are done at least annually because the reputations can and do change quickly (although in some places the fluctuations remain minimal).

Graduate students will invest five to ten years of their lives in gaining advanced degrees; to ignore the massively fluctuating reputations of the departments where they will study is exceedingly unwise. The most competitive graduate programs in the US usually admit about 15-20 percent of their applicants. Most universities now have websites that give data on the number of applicants for each grad program, the number accepted, the number registered, diversity data, and sometimes their completion rates. Note 
that the most highly recommended programs in the US usually register less than 50 percent of the students they admit. ${ }^{1}$ For example, during a recent five-year period UCLA admitted about 30 percent of the applicants to all graduate programs and professional schools; they registered about 50 percent. More specifically, the History Department admitted 23 percent and registered 32 percent. The Physics Department admitted 20 percent and registered 34 percent. The Gender Studies Department admitted 16 percent and registered 38 percent. The UCLA graduate division maintains all those records and allocate resources accordingly.

\section{Becoming a Graduate Student}

What is called graduate school in the US is a rather distinctive instance of 'invented tradition' invoking multiple imagined genealogies, perhaps a caricature of what might have occurred centuries ago elsewhere (Hobsbawm and Ranger, 1983). While much variation in graduate programs has emerged over the last several decades, many programs are very similar to those in place 50 years ago. Those practices have strong affinities with those in medieval religious organizations with a strong focus on discipline. Reading the Rule of St Benedict ${ }^{2}$ on the first year of a novitiate in a monastery will resonate with anyone who has experienced the first year of graduate school. ${ }^{3}$ Anthropological studies of prolonged initiation rites itemize many of the same features that are found in religious and military organizations: prolonged isolation under the control of rigid taskmasters demanding strict obeisance in performing the most basic physical and mental activities, separating the initiates from their previous social worlds and identities, while informing them that all they had learned earlier now requires fundamental revision. The new truths must be absorbed as canon; upon successful mastery the recruits emerge in society as fully initiated and transformed into a new kind of persona (Traweek, 1988).

Put differently, graduate school in the US can be, at the very least, a rather constrained environment. The conventions are simultaneously comedic, pathetic, highly annoying, very harsh, and sometimes far worse, even in the newer, more interdisciplinary fields. Of course, most of the faculty members received their doctorates in traditional departments. As with other strenuous experiences, it helps to have a robust sense of humor and a resilient web of relationships to assist the students in enduring the challenges that seem designed, like military 'boot camps', more to test their physical and mental health than introduce them to new ways of thinking (Ong, 2002, 2005).

Many faculty members see their job not as mentors, but as judges, deciding who should stay and who should leave, a practice known in the US as 'weeding out' the putatively unqualified. These are not idyllic 'ivory towers' echoing with leisurely abstract conversations. Students spend the first few years of graduate school in the US taking intensive seminars to learn the current scholarship on their general research topic area, as well as learning currently 
respected topics, modes of inquiry, research methods, and specific interpretive or analytic strategies, usually learning only those characteristics in one of the subfields within a traditional discipline. Typically, they also learn to scorn other approaches, such as those taught at another university in the same field.

During that period, the students also select research subfields of particular interest, prepare to take written examinations in them, and identify possible dissertation topics. Those exams are taken about the time they complete the required set of seminars. Sometimes students are awarded a Masters' degree upon successful completion of those exams. Many doctoral degree granting programs do not award Masters' degrees or do so only if the student is leaving the doctoral program upon completion of the written exams, as a sort of 'going away present'. After passing the written exams (sometimes called comprehensive or qualifying examinations), the student then writes a dissertation proposal defining a dissertation topic and outlining a probable argument. Typically, the proposal writing process takes about three to six months, resulting in a 25-50-page description of the project, including a review of the scholarly research relevant to the topic, situating the project in the context of ongoing debates. Often this section of the proposal becomes the first chapter of the final dissertation. Drafts of the proposal are shown to the dissertation adviser and the other members of the student's advisory committee. When each member of the committee decides that the proposal is adequate, an oral examination about the proposal is held.

The oral exam often is called a proposal defense. At minimum the defense is attended by the graduate student and the advisory committee. In a few departments the student can request an open defense, inviting fellow students and other faculty members. Usually, the defense is the first time that all the advisers and the graduate student have met together. The proposal defense begins with a 15-20-minute description of the proposal by the graduate student. Then each member of the committee asks questions about the proposal and makes suggestions. The various advisers also discuss how the parts of the proposal fit together, how the dissertation research and writing process can be funded, and the feasibility of the research schedule. Often the student has defined an intellectual project that might take ten years to complete. The committee can advise the student to divide the long project into parts: a dissertation, a post-doctoral research project, and several publications. It is not unusual for a student to be required to reframe part of the project before the proposal is accepted. After the dissertation proposal is accepted the student becomes 'advanced to candidacy' and is called a 'doctoral candidate'. A doctoral candidate is no longer required to take any courses or any examinations.

Until about 20 years ago, students were not discouraged from taking many seminars, studying more languages, and exploring more ideas very carefully. All that has changed. Taking too many seminars, postponing the written exams, and working a long time on the dissertation proposal are considered serious warning signs that the student is not sufficiently self-motivated and 
self-governing to do independent research. I have become known for being willing to work with such students. Many of them have experienced graduate school as triggering massive loss of self-confidence and much confusion about how to present themselves as a knowing subject. They do not see themselves as self-employed entrepreneurs. I find most faculty members, especially the very ambitious ones, are quite uncomfortable with such students. They will have shunned the students they found unsuitable and sought those most likely to finish their work in a timely way with as little need for attention as possible.

\section{Selecting Research Topics, Pedagogies, and Mentors: Disciplined or Entrepreneurial?}

In addition to taking seminars, passing exams, and defining a distinctive research project, graduate students are engaged in an intensive, immersive, transformational apprenticeship, learning to become an epistemic subject, someone who knows in certain ways, and an academic, a certain way of being in the world. That experience would be typical of graduate students in many fields in universities around the world during the last two centuries. How has that changed in the last few decades and what is distinctive about being a graduate student at UCLA?

One feature of the experience that has not changed much is the stylized process of selecting the dissertation research project. The student is expected to focus on a quite specific topic, usually limited in time and space, although showing how that project is related to larger concerns. Then a dissertation proposal is generated, hopefully showing how the specific topic, interpretive approach, and mode of inquiry all contribute to, revise, and/or fundamentally challenge the existing scholarly debates in at least one research field. Hopefully, there is room within all those constraints to design a project that is situated at the intersection of each student's interests.

The structure of a dissertation proposal varies little between research fields. Any university graduate student should know how to understand, evaluate, and generate this template because for 150 years it has been the standard form around the world for evaluating new ideas and new research in many fields. This is a fundamental skill. Most of those making crucial decisions about access to resources in academia know only this form and do not know any critiques of this form. That is, in the context of major ecological changes in universities, the demand for this template remains unchanged. It is a powerful form of tacit knowledge, rarely taught explicitly. Many advisers are quite impatient with students who do not already know how to practice the prevailing templates in their subfields. As students learn to generate what is expected, often without explicit guidance, they also learn to not challenge the tacit assumptions embedded in the templates they are struggling to learn.

Sometimes a research field has converged on a set of questions and formed consensus about the sensible hypotheses, appropriate research methods, and 
useful analytic/interpretive strategies. In that case those issues usually are addressed only briefly and in a formulaic manner. In fields/subfields where so much has stabilized it often is difficult to find the content of that convergence/consensus, or to determine how that convergence occurred and how that consensus was formed. Nonetheless, it is very useful to identify when/ where this has happened and why. In such fields it is very important to learn if questioning that consensus is considered reasonable, and if so, by whom. I recall physicists telling me that when they raised certain questions in grad school their mentors would say that those were questions for mathematicians or philosophers, not physicists, and sometimes advise them to consider making such a move.

There are always research areas with scholars from many countries working on the same themes with similar queries, modes of inquiry, and interpretive strategies, such as the pursuit of the 'standard model' in high energy physics since the 1960s. Usually, these thematic concentrations do shift over time, but in some fields those changes are occurring much more frequently during the last 50 years. Paradoxically, in many of the rapidly changing fields there is not much interest in the processes of change, with much more attention given to the current canon and doxa, no matter how short lived. I have been intrigued that few students are interested in those processes of change that preceded the present concerns. Many faculty members are very clear that they too do not want students to study earlier work in a field which many see as a collection of 'mistakes' or worse. Most graduate students believe teleologically that the earlier ideas must have been wrong, since they were replaced. I first found that thinking among physicists, but now see it is widespread, although historians, of course, find such Whiggish ideas laughable. Most faculty members only want the students to learn the current ways of thinking about their subject, not how those practices emerged. That is, they are being taught canons, however recent, not how to change their minds.

Pedagogies for originality are an interesting paradox. Hence, I suggest to students that it is in their self-interest to work with people who are teaching how to examine assumptions and formulate questions, debates, modes of inquiry, and interpretive approaches, not just making clones by teaching doxa and canons, and not making experts by teaching fixed skill sets, however exciting, complex, or authoritative they might seem. Later when the new doctorates are applying for academic jobs, interviewers will ask about their future projects; they will want to know if the former students can only think of expanding their doctoral dissertations to encompass new cases, however original they might have been. Instead, they must show they know how to continue making new knowledge in new directions, not merely expanding, much less replicating their existing knowledge base. The students are learning how to live within a double bind: the immediate goal in graduate school is to learn the canon and generate the assigned work frequently; success beyond 
the doctoral degree will depend on knowing how to anticipate new lines of inquiry and how to pursue them.

Most students do not realize that faculty members are seeking good graduate students and post-doctoral researchers to enhance their own careers. A more elusive, traditional goal for faculty members was showing the wider academic world that they were able to guide the best of the next generation of scholars. These days professors need such students to populate their graduate seminars and to show their universities that they are advising the requisite number of doctoral students, much like they should be seen as having generated new publications at an 'above average' rate. Gaining access to graduate students is achieved by participation in the graduate admissions committee. There is an ongoing competition among faculty members for those admitted students increasingly seen as 'the best' (according to criteria that might not be clear) and growing avoidance of others whose reputations have declined since admission. The focus is on students who do excellent work at a good pace.

Some faculty members will try to capture such students working with other faculty members. Typically, there also are factions among the faculty members, which we discussed at length in Chapter 3. This affects the graduate students in ways that might not be discernible to them at first. Some gradually learn that they have been working within a faction; some enjoy the clear identities, loyalties, and hostilities that a faction provides; others will not. The graduate students can also play an active role in the factions. Sometimes they display their membership and loyalty by openly showing contempt for any work their adviser has scorned or widely circulate what they take to be their advisers' bon mots. A Stanford colleague once described that process as very old factional practices: hordes of young people are sent to trash the resources of other groups while other hordes stay behind to protect their property from the other groups' hordes. It is both pathetic and violent, a useful device for recruiting certain kinds of students and teaching them the power of belonging to a faction, or not. Pacifists and other outliers will not be impressed; some will be horrified. The collateral damage is large.

In some programs there is a focus on mentoring, professional development, and treating the students like junior colleagues. The alternatives are far more common. The problem has many features: how do students learn to select their advisers and dissertation topics, how do faculty members decide to begin or stop working with specific students, and how do students see their responsibilities to their dissertation committee members? It is in those spaces that harsh pedagogies, abuse, and harassment abound. The old hierarchies persist even among those who might appear on the surface to represent something new. At informal gatherings I have heard my colleagues discuss when they 'allow' their graduate students to address them by their 'first names'. Some say they make that transition after the student passes the dissertation proposal defense; others prefer to wait until the dissertation is complete, while 
yet others insist that it is best to not lower that barrier until the former student has tenure. Some are surprised by all that and say they have never addressed their own teachers informally and do not want their students ever to do so. The hierarchical imperative in academia is rarely challenged, no matter what other kinds of political views the academics might hold.

In the US, students, not faculty members, select their own advisers (dissertation committee members). In each university there are various categories of dissertation committee membership; students need to learn the local definitions for each category, as well as the regulations for the minimum and maximum numbers for each category. Ideally all dissertation advisers provide the following for their doctoral students:

- Intellectual support and detailed information about resources generating an effective dissertation, while also

- Showing students how to generate work that is original, making crucial contributions to current debates, revising positions in those debates, or reconfiguring the debates, not simply 'filling gaps' imitatively.

- Giving access to their meshwork of colleagues who specialize in the student's research areas.

- Giving very strong recommendations for access to funding, postdocs, jobs, and publication; and

- Giving detailed, prolonged advice on job and funding applications, as well as publications in each of their fields of expertise.

We all must select our mentors and advisers carefully with those criteria in mind. There are few explicit guidelines or regulations for graduate student advisers. In most departments only dissertation committee chairs receive any acknowledgment or credit for such mentoring, so some faculty members feel no responsibility for doing more than the minimum. With diplomacy, students can change their dissertation committee members, but only up to a point; after that, the current chair of the committee must approve any changes, including their own removal from the committee. Although changing committee membership can seem awkward, it is far from rare, as exemplified by the fact that everywhere I have been employed there is a bureaucratic form for changing dissertation committee membership. Nonetheless, the experience is harsh. The process might take a year. Sometimes I have helped to facilitate such moves. Each time I have had other senior faculty members privately tell me how glad they are that I am helping the student, how much they understand the difficulties the student has faced, and they offer detailed advice about how to accomplish the change. However, over the years only one of those people has been willing to 'let their name be used'. Even though the bureaucratic forms exist to make structural changes, the very strong tacit message is to not help advanced students once a faculty member has passed a negative judgment on them. Over the years those of us who do help such students informally learn 
about each other and become allies, learning one another's strategies. Again, outliers form communities at the edge. Some with significant local power turn to the resourceful outliers for help in resolving awkward issues informally.

Sometimes the problems are not resolved informally. A quick glance at blogs and chat rooms conducted by graduate students and postdocs, as well as precariate instructors will provide a rich sampling of scathing satire about their mentors and their experience with neoliberal university practices. An elaborate 'whisper culture' exists about how to survive and finish graduate school and a postdoc with one's mental and physical health intact. In some of the worst cases litigation is pursued. Every department in which Sharon has worked in the US has had litigation about problematic relations between students and their mentors. Almost none of it is officially acknowledged because the 'settlements' require a 'non-disclosure agreement' (NDA). There is an ongoing case in my current department; the university lawyers regularly send me notices that I must not discard any email correspondence I might have had with the relevant parties. The regular notices show me that the cases have not been resolved. The other students are another source of information about the status of their colleagues' cases. I was intrigued to see a few years ago that harassment cases brought by graduate students in the UCLA History Department were being argued not by labor lawyers, the conventional practice, but by human rights lawyers based in another country; perhaps that will be a more effective strategy. The other students were circulating petitions and demonstrating on campus. In most cases such matters are far less transparent.

Internationally, Americans have a strong reputation for litigation. It is useful to know that very few legal cases in the US reach courtrooms; the legal system is a vast negotiation apparatus and invoked as such. The legal negotiators share a set of conventional strategies for reaching stylized compromises. In other societies different agents perform the role of negotiators; usually they occupy positions inside the communities where they conduct negotiations and design compromises. In the US they often are consultants working at the edge of organizations.

\section{Funding Ecologies or Access to the Means of Production of Our Work: Teaching + Research}

Another topic usually discussed only obliquely is defining what we need to do our work and how to get those needs met. In that context it is important to know what kind of resources that normally are part of the various work environments we might find and how that is changing. Then we might be able to find a match between our needs and the various ecologies available to us. Over the last few decades I have found that, contrary to my expectations, many grad students, post-doctoral research fellows, and even some faculty members are not familiar with the economic infrastructures currently available to academics in US research universities, much less our strategic 
responses to these conditions. Such knowledge is crucial. It is important to reflect on why that knowledge is not taught.

At US universities faculty members receive nine-month, not 12-month salaries each year. The exceptions are those doing administrative work, such as department chairs or deans, for which they receive either three additional months of their faculty salary or a new salary for those doing fulltime administration with no research or teaching. (Long ago I was told that if such administrators were getting a new salary, they probably have more power than those getting an additional stipend based on their old salary. At UCLA most chairs are getting an extra stipend, as are some deans.) Many US faculty members seek funding for research during those three summer months for which we are not paid; others do summer school teaching. Some invest the time in beginning new projects that are not yet ready for funding proposals. In academia we do a lot of unpaid work or what I euphemistically call 'investing in ourselves'. In the humanities and related fields there is much less extramural funding available than for other kinds of research. Most graduate students in these fields are taught how to apply for both intra- and extramural funding through a series of applications for each stage of research and writing, beginning with funding for conference travel and preliminary archival/field site investigations.

Typically, there are standardized minimum salary scales in US universities; however, unlike academia in most other countries, there is a huge variation by field and subfield in the normal salaries, as well as in opportunities for increases over time. My colleagues in other countries are stunned by the salary differentials in US academia. The first distinction is that applied research pays, R\&D pays better, and data-intensive science pays best, while basic research pays much less, even in the sciences and engineering. More abstractly: there is a 'market' for applied research and other kinds of research in high demand by society, but not for basic research. That is, for many decades society's interest in different kinds of research has strongly shaped pay scales within US academia. The greatest variation in salary is by research field, gender, and ethnicity/race. Such disparities are receding slowly in US academia, just as in the rest of society. There are fewer disparities in the lower ranks and much more at the upper ranks. There are two current glass ceilings: the first is between receiving the doctorate and getting a tenure-track assistant professorship; on average about 25 percent will be successful. The second glass ceiling is between associate and full professors; the success rate fluctuates significantly by field and university.

Next, although we are hired and promoted primarily for our capacity to do research and gain extramural support for it, many US research universities, like UCLA, only provide supplies for our teaching, not our research This includes everything from paper, copies, and pens to books and computers. Most universities provide airfare for one research conference per year, if that, and no per diem. Most of us work far more than 40 hours a week. The usual 
cynical remark about all these conditions is that we are paid in prestige, not money. Former graduate students who leave US academia usually have larger salaries within ten years, at most, than their former teachers. US university faculty in the humanities and social sciences are not doing these jobs for the great money.

Our extramural research funding supplies little for the mentoring we do for student researchers. Most National Science Foundation (NSF) dissertation and post-doctoral funding programs allot no funding for mentors. If an NSF grad student or post-doctoral proposal is successful, NSF will send $\$ 5,000$ to the university in lieu of the usual 50-60 percent 'indirect costs' given to universities by extramural funding sources. That $\$ 5 \mathrm{k}$ probably will not get to the mentor's department, and certainly not to the mentor. Furthermore, in most departments only dissertation committee chairs receive any 'credit' for their work with graduate students, but not other committee members. Beyond teaching graduate seminars, any mentoring work with graduate students, postdocs, and junior faculty is generally uncompensated and, if acknowledged, it is described as 'service'. Of course, if those we mentor eventually do good work, our own reputations might increase among those who know about our contributions.

In academia our reputation and our meshworks are our primary form of what Bourdieu called social and cultural capital. Much of value circulates through those meshworks, including people. For example, I often begin working with students or postdocs because a valued colleague has introduced us, just as some colleagues have worked with my former grad students. Abstractly stated, colleagues can transmit some of their social/cultural capital to their students, although some do not. After we provide mentoring eventually the student/postdoc will be in a position to do that for other people.

Almost all basic research funding resources in the US are linked to established lines of inquiry. Private foundations in the US fund some basic research on new lines of inquiry. Most US government agencies only support basic research in fields that have been established for at least one generation. Newer fields (such as astro- or bioinformatics, communication, ethnic, gender, STS studies, etc.) have special opportunities and problems as they get established in universities, beginning with the development of new research, faculty seminars, graduate courses, interdisciplinary undergraduate minors/ majors, and graduate programs, ultimately leading to the establishment of interdisciplinary research centers, routinely budgeted research units, new faculty positions, and even departments.

All that needs to be matched by launching new extramural funding programs, new research journals, new conferences, and new series at university presses. The whole process takes about 25 years. The people engaged in building those ecologies (like me) are rarely funded by their universities for doing so, although we might receive some 'credit' for what is called 'institution building'. Sometimes the newer fields have extramural funding resources 
(especially from private foundations or new government funding initiatives) and more publishing opportunities because presses believe there are larger audiences for the more recent scholarship. Often, our colleagues in traditional lines of inquiry are annoyed by and actively work against what they regard as the deflection of scarce resources to ephemera, locally and extramurally, as did two of my UCLA colleagues over a period of years. A few US universities reward the work of building the institutional scaffolding around new lines of inquiry, but most do not.

\section{Working in 'The College'}

Meanwhile, US university positions outside the professional schools are scarce and highly competitive. As noted earlier, since the 1970s it is not at all unusual for 100-300 people from around the world to apply for assistant professorships at major US research universities; at least 25 will be stunningly well qualified. Six years later only about one third of those hired will make it through the year-long tenure evaluation process. The compensation for working in these highly competitive resource-scarce ecologies, aside from the putative prestige: only tenured university faculty members 'in the College' are free to pursue curiosity-driven basic research without fear of losing our jobs if we do not have continuous funding for our research. That privilege is very important. Many other kinds of privilege have emerged in US universities over the last several decades.

My friends in the US professional schools have better salaries and there is far greater tolerance for interdisciplinary work. However, they are expected to maintain high levels of extramural funding, almost all of which is for applied research. Many positions in the professional schools remain unfilled because many prefer to get even higher salaries in industry where there will be no need to seek individual funding. Some very large corporations still maintain their own basic and applied research programs. From my research about funding ecologies at US national laboratories I have learned that many former university-based researchers are vastly relieved to have escaped the need to seek funding personally for themselves, their postdocs, and their grad students. People far removed from their projects negotiate funding for the entire organization; then small committees within the organization determine resource allocation. Similar processes exist in industry, although funding there usually comes from the sales departments, not government allocations.

From friends at US non-profit research centers I have learned that they are 100 percent dependent upon grants, whether from government or industry. Stated bluntly: no current funding means no job, no matter what the history or extent of previous funding. Usually, they spend about 30 percent of their time tending to the grant process. These conditions are characteristic of any 'softmoney' research job. Most of what I know about US industry-based research and development comes from colleagues at Apple, IBM, INTEL, and the old 
days at Xerox PARC: my general understanding is that the research funding tide ebbs and flows with the companies' current profits. Historically, there were some major exceptions, for example, Bell Labs and the Dupont labs, but their invulnerability paradoxically has not survived the transition from manufacturing-based to information-based political economies. By contrast, US university-based researchers are much more like individual entrepreneurs. Most of us are quite accustomed to working in 'scarce funding ecologies' and even take pride in that. I have heard many US university-based physicists react with scorn at seeing in industry and government laboratories what they call 'over-spending' due to a lack of judgment and failure of nerve. Of course, those university-based physicists are working in a much fatter funding ecology than mine.

\section{Hiring Colleagues}

Now I turn to how new faculty members are hired. What is the process for generating and filling a new professorship position in leading (R1) US universities? My colleagues at most universities outside the US indicate that new positions are allocated by universities when someone retires or leaves the university for another position. In over four decades I have never seen or heard of a new faculty position created that way at any R1 university in the US. No positions are routinely bestowed in US universities. Faculty members must invest a huge amount of institutionally unacknowledged work to gain access to positions that will be filled with new colleagues. Learning how to access such positions and doing that work is an important part of a faculty member's job. Most new faculty members know nothing of the process. Over the last several decades there have been significant new developments, showing the ways US R1 universities are navigating new university-society relations. The process also shows how faculty members must act together as an entrepreneurial team in competition with those in other departments to build, maintain, and expand our home departments. Very little happens without such time-consuming activity.

New positions (usually called FTEs for 'full-time equivalent') in US R1 universities are created through two channels. One pathway is a so-called 'faculty FTE'. For example, in the University of California when someone retires or leaves the university that FTE reverts to the UC system as a whole. When budgets are determined for the system, a certain number of new faculty positions are created. Through a complex negotiation process each of the ten campuses are allocated a changing number of FTEs each year. In turn the campus chancellor and provost decide each year how many FTEs to allocate to each professional school and the 'College' (the traditional university). The deans of those units then determine which departments are to receive how many FTEs. The allocation of those quite valuable and very scarce resources are at the discretions of the chancellor, provost, and deans. 
Meanwhile, each department holds annual hiring priority meetings at which the faculty members discuss the most important developments in their field and how the department ranks those developments in terms of hiring a new faculty member to specialize in one of those currently important research areas. The department then generates an annual hiring priority report for the dean, describing how and why the department members reached their decision. For example, simply because a specialist on the history of the French Revolution has retired does not provide a viable justification for the department getting a new FTE or that when an FTE emerges for the department it should be filled by someone with the same research specialty as the retiree. I have never witnessed or heard of such a practice in a US R1 university. The report to the dean about the department hiring also must include a justification for the department getting a new FTE; someone having left the department is far from sufficient. The appropriate reasons include the current and anticipated number of undergraduates and graduate students in the context of highly fluctuating student enrollments for the reasons already discussed. Another is that for graduate students to be competitive in the faculty hiring process they must be educated in the currently significant research areas in the field which means the department must have faculty members working in those areas.

Put differently, the UCLA Dean of Social Sciences oversees ten departments, five of which have existed nearly since the founding of the university and five of which are much newer interdisciplinary fields. About ten years ago, during a time of massive financial restructuring of the University of California the then dean decided that the two largest departments (history and political science) were 'abnormally' large and should begin a long process of downsizing, which continues to happen, in spite of their massive ongoing objections and efforts first to change the dean and then the policy. The two departments had held impressive informal power within UCLA for decades and the command to downsize was shocking to them, although welcomed by many other groups that had had to function for years in their turbulent wake. Any justification for a new Faculty FTE must take a position on such larger ecological issues and explain why any one department should have access to the scarce, important resource of an FTE. The Gender Studies Department has requested annually, but never received a 'Faculty FTE' from the UCLA Social Science Dean.

There is a second process for a department to gain access to faculty positions; those are called 'Institutional FTEs'. Those positions are defined at the discretion of the campus chancellor or the president of the UC system, usually in response to a significant new initiative. One example is the UC President's Post-doctoral Fellowship Program (PPFP), designed to increase the proportion of minority and other under-represented groups among the UC faculty. Through that program a number of postdocs are awarded annually. If any department petitions to hire one of those former Presidential 
Fellows, then the UC President's Office will fund the FTE for its first five years; after that the home campus supports the position. About half the current faculty members in the UCLA Gender Studies Department were hired using the PPFP FTEs, showing a consistent pattern of entrepreneurial activity in the department as faculty members locate and gain access to new resources. Many of the older departments conventionally avoid the Institutional FTEs because they want to focus on Faculty FTE positions because that enables them to define precisely the research specialization of the new member in order of the department's defined priorities.

UCLA also has a few hundred so-called 'endowed professorships' and 'presidential professorships', all of which are Institutional FTEs. ${ }^{4}$ Once one of those positions was designated for research on women; along with several other departments the Gender Studies Department applied for that FTE, and we were granted the chair which was filled a few years ago. Finally, the chancellor has additional FTEs to allocate based upon exceptional opportunities to recruit a faculty member to UCLA. Sometimes that includes the opportunity to hire family members of new recruits, who must be someone of the highest scholarly reputation who would not otherwise be considering a move. Deans sometimes ask departments to accept such an FTE as a favor to the dean. The Gender Studies Department has accepted two such FTEs, but not all that were offered.

In sum, a significant number of faculty positions in the Gender Studies Department have come from a long, carefully honed practice of successful strategic engagements with the Institutional FTEs from UCLA chancellors and the UC President's Office, rather than the conventional Faculty FTEs that are allocated by deans. The other faculty members in the department chose to move their positions from their prior departments at UCLA, as I did, moving from the History Department. About a dozen of us in that department were actively involved in 'departmentalizing' the then existing undergraduate curricular programs in Gender Studies, African American Studies, American Indian Studies, Asian American Studies, and Chicano Studies. As those programs achieved departmental status, many of us moved our FTEs into the new departments.

When I decided to move my faculty appointment to Gender Studies, my former colleagues in History said that seemed too risky. I tried to explain the trends I saw. When I left the History Department it had about 75 regular faculty members; ten years later the university plan is for it to continue reducing to 40 members. Gender Studies had eight members when I joined; it now has 21 and next year will have one more. All over campus the newer, more interdisciplinary programs are growing, as they do at universities everywhere, although widely scorned by most faculty members in the traditional disciplines. Interestingly, some harsh critics of interdisciplinarity opportunistically hold faculty positions in the interdisciplinary departments. The graduate students working with them get very mixed messages. 
Once a department has received an FTE, the hiring process begins, which takes about one year. It begins with an elaborate job description, decided upon by the full department, which is then published in a wide array of scholarly venues, hopefully international, related to the field in question. It is those announcements that elicit so many applications. A full application dossier will include about 50 pages: statements of interest in the position, another about diversity issues, a third on pedagogic commitments, and a fourth on research interests and goals for the next five-ten years. There also will be a full curriculum vitae (at least five pages), three or more letters of recommendation (normally each will be three to five pages), sample syllabi, and examples of scholarly work, including any publications. The process is extremely competitive.

Hiring committees are composed of faculty members from the hiring department. They first narrow the applicants to about 25; at that stage they might conduct brief interviews online or at a conference in the field, narrowing the group to about ten when additional recommendation letters are requested. Finally, a 'short list' of three to four is selected and each of those candidates are invited to visit the campus separately, usually for two days of intensive interviews by department faculty and students, lasting from breakfast through dinner. The candidates will also make up to three presentations, one on their research to the full department, another as an undergraduate lecture, and a third as a graduate seminar.

Departments want to hire people into tenure-track positions who will do well at their third-year review and then in their sixth-year tenure evaluation; failure reflects not only on the candidates, but also on the departments' original hiring decision and their subsequent mentorship. The most important tasks for tenure-track assistant professors are to obtain research funding and publish as much single-authored research publications as possible.

\section{Strategic Mobility and Negotiating}

The changing standards for a strong academic career in the US leads to many people changing jobs every seven to ten years, with substantial improvement in their working conditions at each move. People move to strategically enhance their resources for future projects. In many countries the terms of employment for academics are not negotiable and, in such places, academics often believe that everyone in the same rank is paid the same in every field at every college or university of the same rank. That is decidedly not the case in the US. My friends outside the US are startled that even basic resources are part of individual negotiations in the US. Many universities encourage faculty members to think of ourselves as self-employed entrepreneurs, so we are expected to negotiate for resources as if we were outside consultants seeking a contract with a corporation. However, in my experience at several private and public universities in the US since 1975, I have never seen an individual employment contract. At each place where I have studied and worked there 
are 'academic personnel manuals' that identify some general employment conditions and requirements for each job title. ${ }^{5}$ Because our negotiations with deans are informal and a new dean may appear in a few years, faculty members try to get the informal negotiations confirmed at least in email exchanges.

Third, there are few US universities with unions; some have faculty associations that have little if any negotiating power with universities. That implies that every feature of academic employment is individually negotiable at every career stage, from graduate student, post-doctoral, and instructor positions to full professor and even endowed professorships, when changing positions, but rarely re-negotiable once working within a university. Once employed the changes are more incremental than substantial. I did not know that at the beginning of my career, but once learned I resolved to share that knowledge. At each stage I began compiling more information and sharing that. Decades later I still get requests from strangers to see the list, having heard of it from friends. I only ask that they share something from their own negotiations so I can add to the list; only one has not done so. In our negotiations we must define our priorities; what scarce resources matter most to us, such as time, decision-making authority, independence, money, rank, or other items. We are expected to state that our situation, needs, and goals have evolved since our last moves, promotions, negotiations. We have important intellectual and professional reasons for moving, but we do not want to lose what we already have. We ask for additional resources to enable us to accomplish our new goals. In conducting those negotiations, we learn how the new institution makes decisions, in what way, and at what level. We also learn the normal procedures within each university for accessing resources. It is important to clarify, in detail, current policies, and our own current and eventual levels of access to these kinds of resources. Some may think that itemizing the resources needed to do our everyday work is obvious and/or too petty to discuss. In the US we have learned to beware of that kind of thinking.

\section{Becoming an Entrepreneurial Academic}

I recount my own experience because it is an example of the changes in forms of knowledge making in universities, graduate education pedagogies, faculty positions, research practices, and evaluations, as well as subject formation during the course of a career. The incidents I describe were far from unique which is why I recount them. They were unusual in the sense that I have been part of a hitherto unusual cohort to enter and continue in academia which has worked on hitherto usual topics and epistemic approaches. Together we have moved through academia as a faultline; we were a sign of ruptures with past practices that first were seen as fleeting aberrations and then we were considered a trend. Gradually, we gained access to and even control of some resources, which is when we became annoying. Finally, we came to represent 
a viable alternative and even new lines of inquiry and practice. Various groups try to make use of us.

When I finished my undergraduate degree at UC Berkeley (UCB) it was still very unusual for women to be accepted into doctoral programs; we were expected to get Master's degrees and then teach at the best secondary schools or perhaps lead programs at local cultural institutions. On the recommendation of my adviser at UCB I went to San Francisco State University (SFSU) for a Master's degree in history. My adviser there was a foreign student completing his doctoral degree at Stanford. Later he taught at the University of Texas and Brown before becoming provost at the University of Pennsylvania, the president of the New York Public Library, and finally head of the Carnegie Foundation. He became a powerful and important academic leader in the US, displaying the strategic and entrepreneurial acumen that I had witnessed at SFSU. I left SFSU to do as women with Master's degrees were then expected to do: teach at interesting secondary schools; one was a site of great privilege and another was profoundly not. I became intrigued by that juxtaposition and worked in an assortment of such positions in an effort to understand more about the educational inequities that accumulate around the other kinds.

Eventually I decided to return to graduate school for a doctoral degree at the same time as several friends who all had gotten Master's degrees. All of us had reached a point in our work in which we realized that we would have far more opportunities for promotions and to make changes if we had doctorates. It also was the moment when US graduate schools had been forced by litigation to not discriminate against women applicants. We returned to school to get doctorates, but we did not intend to become academics, although all of us did. I knew that I wanted to stay in my community in which I was a busy activist, so I only applied to two schools, both within an hour of where I lived; I also applied for extramural funding. I got the funding and admission to both schools. One was for a very highly regarded program at UCB and the other was for an unusual interdisciplinary program at UC Santa Cruz (UCSC), then about ten years old. Several friends in academia said that if I were to 'invest' in getting a doctorate, I should get one with the most accumulated prestige which was not available at either UCSC or the doctoral program there.

The day I visited the UCB History of Science Department to meet two prominent faculty members, I first encountered a visiting assistant professor who explained some gender problems at UCB, including a then new national government investigation about discrimination in five UCB departments, including that one. After that I was far less interested in UCB. I then visited the program at UCSC, which had intrigued me because it was run as a collective by its students. The application was a dissertation proposal that explained why the proposed project could not be done within any one discipline. Clark Kerr, the president of the University of California when several new UC campuses were launched, had planned for UCSC to demonstrate that a public university system could include a first-rate liberal arts college. The History 
of Consciousness doctoral program (Histcon) existed there because many of the more prominent faculty members recruited to UCSC in its early days had wanted some graduate students to mentor individually, in what they called a European tradition; Histcon was the efficient institutional solution. The day I visited I spoke with a faculty member whose last name began with a B because it was the first one on the list. He asked about my research interests and I explained I had a problem: I wanted to do an oral history project on research practices at a lab where I worked; the scientists had accepted my plan, but I had learned they had three very competing versions of their past. My interlocutor said that then they must have competing versions of their future; I happened to know that they did. I asked if he had ways to study that process and he did; I was hooked. The men at Berkeley had said they would help me find the correct version among the three claims.

I was an activist and a rebel; I knew that if I returned to UCB I would spend a lot of energy and time trying to change things. I realized that if I went to UCSC I would be helping to build something new. Later I learned more about Gregory Bateson, why he was at UCSC, and that he was a Regent of the University of California. Together we designed a course of study for me which would be primarily tutorial, supplemented by many reading groups organized by fellow students. We would compete with each other about who was doing the project least acceptable to academia. We all planned to return to our community activism with new ideas. Our indifference to futures in academia enabled us to ignore cheerfully the conventional expectations. Faculty members whose knowledge I sought declared my research topic very interesting, but not academic; I was not deterred. As anomalies my student colleagues and I were learning to survive successfully in the margins of academia, being innovative and original, all within an experimental ecology designed by the UC president which we were glad to appropriate for our own purposes. Oddly, almost all of us became academics; academia was changing more than we realized. In retrospect I realize that in today's academic discourse we could have been labeled 'social entrepreneurs' who were together learning how to modify academia. UCSC's and Histcon's reputations would rise over the next few decades while the UCB department's gradually waned, as has UCB's which made some extremely unwise investments in a new tech campus that foreclosed, leaving UCB with massive debt that still strongly affects everyday life on that campus.

Now I know that to ignore how the reputations of the departments where we will study or work are changing is exceedingly unwise. All this is much more obvious now than it was when I decided to return to school for a doctorate, but the signs were there, in retrospect. In my choice of UCSC over UCB I was far less anomalous than I realized. My fellow Histcon students and I could be seen now as having taken an intensive course in academic corporate entrepreneurialism. I think we succeeded because we had no sense of risk; we did not intend to stay in academia and thought we were only changing our local 
ecology to make it a bit more accommodating for our needs. We were not taken very seriously because we were students in a cheap program designed to please a few faculty members who demanded a few graduate students as part of their recruitment. It seemed a lot like some of the start-up companies I knew in Silicon Valley, over the hill from Santa Cruz.

After I had been in the program two years some UCSC faculty members tried to get Histcon closed for reasons that had to do with their ambitions for its limited resources, not the program. A few of us students decided to negotiate with the chancellor; we asked him to consider our proposition: would he keep the program open if we could hire an internationally prominent scholar to head the program? He was willing to fund such a recruitment, but warned us that it would be difficult; we managed to do as we planned. The new chair of the program was Hayden White; as part of his recruitment he got the authority to hire two junior faculty members. Donna Haraway and James Clifford soon joined Histcon; both became internationally prominent scholars. The three of them got resources to hire more faculty members. The individuals who had tried to get the program closed became sycophants. Later in my career I saw the same process repeated in various contexts.

As a graduate student at UCSC I had audited an anthropology seminar at Stanford. The professor said that a Stanford student and I should combine our papers, submitting the result for publication. After a lot of effort, we managed to get an article published. ${ }^{6}$ Ever since the article was published whenever I have been hired or promoted by anthropologists, historians, gender studies scholars, and STSers I have been asked to identify 'the pages I wrote' in that co-authored article. I have been told that to not identify the pages would mean that the article would be considered only as 'service to the profession', not scholarship. At every stage of my career since then in each of my four fields I have been told the same about collaborative work. For the last 20 years I have engaged actively in collaborative work; this book is yet another example. Over the last 50 years many fields in academia have become much more engaged with collaborative work, but many others have not accepted the practice among either students or colleagues. They are still focused on an individualistic, private property understanding of knowledge making, even though they might support collective action in public political arenas. I think the contradiction powerfully undermines both aspects of their work.

When I arrived at MIT as an assistant professor (1982) the anthropology department chair explained to me that one of the reasons they liked my application was that I already had begun learning how to participate in an array of normal academic activities: current debates in the field, workshops on current debates, organizing conference panels, department administration, and teaching. He pointed out that when he began his career, older faculty members were expected to mentor younger faculty members as they learned those practices. He added that by 1982 no professors in the MIT anthropology department had time for that much mentoring of new faculty, so 
they wanted to hire people who had begun to get that mentoring in graduate school, plus had shown signs of learning to learn those strategies on our own. Later my MIT STS department chair told me the same thing. (I had a joint appointment in those two departments.)

\section{Becoming Evaluated}

For both the 3rd year midterm and 6th year tenure reviews assistant professors at R1 universities are asked to provide the names of three to five people outside the university who would evaluate their work. Since I was in two departments, Anthropology and STS, I was expected to give that many names in each field, and it clearly was important for me to name well-known people. (In the US the candidates' dissertation committee (DC) members will not be asked for letters of recommendation for either the midterm or tenure evaluations. DC members are seen as too eager to see their former students tenured.) The MIT promotion committee would add three to five more names for each of my fields. I had learned from studying physicists that assistant professors should build, strengthen, and maintain their own ties with prominent and other post-tenure specialists in the fields relevant to their research. As a shy person I felt very uncomfortable engaging in such activities; however, I knew it was necessary and not doing so was self-defeating, not seen as a sign of modesty. I volunteered to give many presentations at conferences and other venues, always introducing myself and my work to those whose work inspired me. The process has always felt awkward, but gradually I developed some skills in self-presentation. I noticed friends in other countries could rely on much more predictable set of introductions from their advisers. It seemed that in the US we all had to become adept at one- and three-minute 'elevator pitches' and seven-minute 'pecha kucha' presentations about ourselves and our work. A 15-minute conference presentation was a luxury.

At my third-year review I got the highest evaluation: promoted at MIT to associate professor without tenure, with the encouragement to prepare for a tenure review three years later. My dissertation had been revised and accepted for publication at Harvard University Press. I got funding from the Fulbright Foundation to continue my research in Japan. I got excited at one stage when a different university press approached me about another book on a topic of strong interest to me: changing practices in the visual representation of data in the sciences. My MIT mentors said that was not appropriate; I was to develop a reputation for being an expert about something specific. I began to think that what would get me promoted at MIT would enable me to leave. I wanted to find someplace where I could set my own intellectual agenda.

While in Japan one of my graduate school advisers suggested I apply for a position in the small, but internationally recognized anthropology department at Rice University in Houston, Texas. To some of my colleagues it was clear I should stay at MIT because it had a higher rank as an institution. Someone 
also told me that if I left before getting tenure, forever after everyone would think I had been denied tenure. However, the Rice University anthropology department was far higher ranked than the MIT department and the MIT STS department was so hierarchical that it would have taken me another ten years, at least, to have a decision-making role. I moved. A year later at a conference the chair of my MIT department walked up to me at a large gathering, gave me a big hug, and chatted warmly for about ten minutes. Part of what he said was that he knew everyone thought I had been denied tenure at MIT and he was signaling that was not the case to all those present. Then I noticed all the surprised faces.

Most of what follows addresses the evaluations of my work as an associate professor at UCLA. In principle, the next major stage of the 'becoming an academic' process is promotion to full professor, a step I have never achieved in the US. (Over ten years ago I was invited to be a special visiting professor at a university in Sweden; I was evaluated as qualified to be a full professor there.) What follows describes what I take to be the reasons I have not been promoted. I do so not because I think my situation is unusual, but rather because it is not. In the US the unpromoted are called 'stalled associated professors' with the acronym SAP, which also is a colloquial expression with several meanings. The Cambridge Dictionary describes a sap as 'a foolish person', and for the Oxford Dictionary a sap is 'a stupid person that you can easily trick, or treat unfairly', providing this example: 'The poor sap never knew what was going on behind his back'. At many US universities there are programs to assist SAPs in overcoming their 'stalled' condition. I receive their announcements regularly and save them all for my research. Like so many designated SAPs, I do not think I am 'stalled' and consider myself quite active and rather accomplished. Why might my university disagree so emphatically and punitively on this point?

As an assistant professor at MIT I was told that for tenure, promotion to full professor, and any subsequent honors it would be necessary to change the way people in a field think about an important topic. I was not surprised by the criteria. My undergraduate and graduate school advisers all had achieved that. I have further examples because several colleagues and former students have done that too: their first significant work did not resemble their second, and so on. A few of them also have engaged in 'institution building', launching some new infrastructure for a new kind of knowledge making, as I too have done various times. Some of my exemplars would not have been considered particularly productive in the contemporary modes of evaluation by 'metrics' (counting), but they have regularly changed people's minds while building academic infrastructures for others to use. Some of them also had setbacks and then pushed ahead. I still find them excellent role models as I have tried to do the same. Others have told me to become expert at something and stick to it; other colleagues told me my first book was so interesting I should keep doing that kind of work. I knew that instead I wanted to keep changing my 
mind, and hopefully others' as well. To many that would be called a focus on innovation.

The repeated promotion denials at UCLA have taught me that what I was seen as doing was not valued and much of what I was doing simply was not even noticed. Eventually I decided that the various institutional evaluations of my work, along with many other SAPs, were radically inappropriate; I resolved to examine how that could have happened, rather than simply rebut the claims the reviews included, as we SAPs are always invited to do. I resolved to interrupt some ongoing research projects at that time and investigate the problem. As UCLA gained higher rankings why did it rank me lower? I now understand the problem lies with some university strategies for 'questing excellence' that do not conform to mine. They are seeking rapid production of certain products. They want another kind of 'entrepreneurship' than the sorts I perform.

Several years ago, I belonged to a group of SAPs at UCLA who launched a systemic study of the problem. With the help of a legislator overseeing the California State Board of Audit that annually evaluates the University of California (UC) budget we got access to relevant data for the entire UC system of ten campuses. We learned that the SAP problem was greatest at UCLA and within UCLA it was greatest in the social sciences; the most extreme problems were in the history and sociology departments. SAPs were not only not promoted; if we received extramural awards or offers of employment elsewhere, we did not receive salary increases or 'retention offers'. The criteria for promotion that were used for our cases had not been followed in many cases that had resulted in promotions. Few of us had institutional mentors advocating on our behalf, as had the promoted ones. We only had prompts telling us what we had not done. As you might have surmised, women and other groups under-represented among academic faculties are far more likely to be SAPs.

Eventually we submitted a report to the university, including deans and department chairs, and some new deans gave extensive pay raises to quite a few SAPs across campus. The then vice chancellor for Academic Personnel acknowledged that certain criteria for promotion would be applied equitably in the future, such as articles being accepted in lieu of books, and so on. Recently I heard mid-career colleagues in my current department, all advancing quickly by performing the expected kind of academic entrepreneurship, smugly insist on 'the second book' criterion, as if that were the only signal of proper achievement.

Part of the problem is with a set of forms used to collect data for decision making about promotions. Several years ago, UCLA began to require a single format for representing our work as faculty members. The first half of my career there was a wide array of practices that clustered by discipline and subfield; part of the act of 'belonging' was knowing what to include and ignore in generating a 'curriculum vitae' (CV), a learning curve that began 
in graduate school. At first, I chose to submit a CV of my own design. More recently I was told the current template is required. There is no space on the form for much of what I do. That is how I have become designated officially as an 'inactive scholar'. As discussed in Chapters 2 and 3, UCLA has adopted a nested set of platforms and algorithms for admissions, budget management, teaching, and faculty evaluation, among others. They have helped to build an elaborate system of metrics, used to define excellence with a focus on quantity, not quality. Clearly, my work has been found wanting by those particular systemic metrics. As I have shared that story with others around the world, I have been given similar examples about scholars in many fields at various career stages in different universities. We have consulted with prominent scholars who are dismayed by the kinds of work being thwarted by inappropriate reviews.

As part of my protest to the decision about my work some years ago that I found so shocking, I had resolved to not update the same forms used as part of that review. It is the assumptions embedded in the platforms that structure the review apparatus that I am challenging with my current scholarship. Clearly my work has not been found wanting by the international scholarly society in my field that bestowed upon me their Bernal Award for careerlong achievement, the two journals which have published two articles by me this year, the university press which will publish a chapter by me in an edited volume next year, nor my co-author or the publisher of this book. The considerable accomplishments of my former students are not taken into account, although I continue to serve as a mentor for them, as well as several others.

I have received the faint praise that those current publications are evidence that my research has revived; it never waned, so it has not needed reviving. As I had described in earlier reviews, following my recovery from an intense health problem in the late 1990s, I had begun exploring collaborative work in a series of projects, some of which have proved far more interesting and rewarding to me than others. Collaborating is of course not without challenges and I have had a steep learning curve. One colleague said to me that he was very surprised that I had ever agreed to work with one of my collaborators; that is how I learned what many already knew, but I had not. I should have investigated the person's informal reputation, but had not. That reminded me of when academics worldwide had begun asking me what it was like working alongside two colleagues; I was surprised by the question and asked what was meant. My interlocutors told me that both people had very problematic longstanding reputations and many had wondered how I had managed. We discussed the depths of my naivete. I was reminded yet again of the informal meaning of SAPs, as well as why so many scholars work alone. In Chapter 6 we address the role of gossip, informal communication in professional meshworks, in recalibrating the reputations of even the most prominent scholars, noting another feature of quality that is not captured by metric evaluations. 
News of the harsh consequences of my naïve choices has spread. As I mentioned earlier, many know I have been scorned before they begin to realize they themselves are beginning to be marginalized; then they seek suggestions. I often get email messages from academics, including graduate students, within and beyond UCLA, even from other countries, intrigued by how I have survived. They want to know the strategies for continuing, rather than leaving. I have worked over the decades with several people who were on the verge of leaving or being dismissed; all are now doing very well. I ask them to spread the word. Although we can do that successfully, none of us has been able to dislodge the problems that marginalized us; indeed, those problems in academia seem to be increasing. Some who have succeeded quite impressively and are recognized internationally are still reluctant to acknowledge the setbacks, the tenure denials, the refused promotions they have endured. We all need to acknowledge openly such experiences, not only to inspire others, but to make some substantial changes, such as we discuss in Chapter 6.

As Maria Ong has long argued, there are 'strategies for success' among those who are outliers (Ong 2002, 2005; Ong, Smith, and Ko, 2018). I too began to study the survivors who succeed without conforming to the new metrics. There are many who have found that strategies for surviving at the edge of academic institutions also can be used for our epistemic explorations at the edge of academic assumptions and canons. We have learned many times that ethical hypocrisies might have epistemic, methodological, and political correlates. If we are engaged with tasks and colleagues that inspire us, along with communities of practice that sustain us, we have learned how, intellectually, socially, and personally, to endure the scorn and condescension of others. Of course, we also bear the scars of accumulated anxiety and melancholy, as well as the comfort found among the scorned and those who support us. Increasingly, the survivors are willing to tell their stories about the contradictions embedded in the academic work done in the name of 'metrics'. We had stopped wanting to be what we were supposed to be, the right kind of subject in the metric economy of the gig university. The precariat is refusing to accept the scorn as justified. Yet others see the process and refuse to embrace it, even when it supports them.

I have been intrigued to note that the most recent evaluation of my dossier does not acknowledge my statement of protest against the formats, the templates, the algorithms, my refusal to outline my accomplishments in those templates. It is simply noted that many items were missing in the required dossier, although I had clearly stated in my letters enclosed in the dossier why items were missing and then explained my position, as I am doing here, and as so many others have done in similar accounts. From the field of Whiteness Studies, we have learned that the violence of racial privilege is sustained by the silence about the means of production of its own privilege; it is maintained by not acknowledging power explicitly while practicing it tacitly. To call 
attention to the processes of privileging is the beginning of unraveling the power of tacit practices.

My protest is not against any individuals. My focus has been on a cluster of strategic practices that UCLA and other neoliberal universities have pursued intensively for the last few decades, clearly generating great rankings and much success of the kinds that can be measured by its chosen metrics. It is my contention that it has done so in ways that needlessly but actively thwart other kinds of success, such as mine and many others, leading us marginalized to find new strategies. Of course, that provides us with more epistemic resources, too. I have spent the last several years determining how to specify how that has happened and how to address it, institutionally, epistemically, and collectively. I have no expectations that UCLA will change its dominant strategies for success, but I do hope that those evaluating my work will remove the obstacles inappropriately put in my path and many others as we continue to achieve success in other ways. This book will go into my next dossier.

Conventionally we think of knowledge being made by knowing subjects whose epistemic privilege has been individually generated and maintained through a series of certifications from disciplines and institutions, while demonstrated by the circulation of original interventions in scholarly debates, all published in venues with access restricted by rigorous peer review. I study those subject formations, the fashioning and performance of the required discursive strategies, and the global assemblages of highly stratified knowledge infrastructures, as well as how they change over time. How and why do certain disciplines, institutions, debates, venues, and infrastructures in certain places accrue the authority of bestowing the privilege of abstraction, classification, and universality, and others are saddled with specificity (Traweek, 2021b, 2022)? How is knowledge made at the edge of that assemblage of academic power (Traweek, 2021a)? What are the success strategies for making ideas and careers as intellectuals while occupying the margins $(\mathrm{Gu}, 2016$; Holbrook et al., 2018; Holbrook, 2021; Murillo et al., 2012)? Clustering at epistemic faultlines where conceptual ruptures are expected, those at the edge form robust meshworks, webs of relations for circulating their ideas and making them more robust and resilient. Many have taught us that embracing our mixtures, borderlands, margins, and differences can provide an excellent standpoint for challenging the stylized assumptions, narratives, and practices of epistemic privilege, entitlement, and authority.

In an earlier book I outlined how people come to be the right kind of person to be a physicist, learning by listening to and then telling certain kinds of formulaic stories at each career stage, rehearsing a certain kind of normativity that enabled them to fit well into certain kinds of collaborations with certain kinds of goals, and even to lead that community of inquiry (Traweek, 1988). I pointed out that some were not telling the right kind of story. In talking with leaders, I asked about their exclusionary practices. One said it always 
is important to keep some outliers, because they just might be right. When I asked about the proportion he demurred. Over the last few years a group of researchers in the US and the UK have written about the importance for 21stcentury corporations of a certain kind of difference, described as 'positive deviants', 'loyal rebels', and 'rebels with a cause'. I am amused to learn that after all these years as an outlier among others, we are a kind that is considered just what corporations now need: an impatient critic who cares enough to want the organization to improve (Dahling and Gutworth, 2017; Heracleous, Terrrier, and Gonzales, 2018; Heracleous and Robson, 2021; Petrou et al., 2020). However, I harbor no illusions that UCLA is likely to notice. The corporate model espoused globally the last 25 years is not the 20th-century model now being intensively pursued at UCLA. The newer model has a flat organizational structure and rewards lateral ties with other companies in the same industry and beyond; it nurtures its own ecology. Laurel Smith Doerr (2004, 2005) studied how women biologists moving into such companies have thrived far more than their women colleagues who have stayed in the hierarchies of academia. While it might appear we are recommending that universities merely shift, belatedly, from being 20th- to 21 st-century corporations, that is not the case, as will become clear in Chapter 6 .

The paradox of corporate entrepreneurship has been widely addressed by many commentators on various sectors of information-based global political economies, not least of which is the precarious world of gig workers. In the context of US academia there can be stark tension between being a good corporate worker, adding to the productivity metrics to improve universities' rankings, and being another kind of good worker, developing new lines of inquiry and building new infrastructure for them. The corporate academic accepts engagements with the new algorithmic governance, managing with metrics, but only as long as it does not challenge the conventional allocation of resources. Another kind of academic accepts the challenge of making new kinds of research and teaching, not just more research and more students, but at risk of not meeting the productivity demands.

The test of that performativity is at times of evaluation and hiring. The struggle to be the right kind of performer is most apparent at four stages: when graduate students are evaluated for promotion to doctoral candidacy and then hiring as tenure-track assistant professors, followed by the tenure evaluation and then consideration for promotion to full professor. As those evaluations approach mental and physical health problems accumulate, sometimes in debilitating ways, including the 'imposter syndrome', all widely documented on internet blogs and chat rooms. Zorn and Boler (2007) identified five factors which cultivate the syndrome in universities: scholarly isolation, disciplinary nationalism, aggressive competitiveness, the valuation of product over process, and lack of mentoring (or bad mentoring). Those troubling states, always on display in US academia, serve as powerful prompts to become the 'right' kind of academic subject. 
There are other displays that provide powerful motivation to perform appropriately. At any one of those four major evaluation points the aspiring academic subject is vulnerable to failing. Those who have not gotten an assistant professorship in their first or second cycle of applications try to conduct post-doctoral research on term contracts with various levels of prestige. The more precarious route is to take short-term teaching assignments at multiple institutions. Such teachers are essential to US universities; since the 1970s surveys indicate that about half of all undergraduate students (and about one third of all courses) are taught by such precarious workers (AAUP, 2017; Baldwin and Chronister, 2005). Few have access to union membership and in most cases, they cannot be rehired to teach again after about five years. Every year their ranks are increased by the most recent doctoral degree recipients who have not yet gotten a tenure-track appointment. They are the surplus labor force of academe. That practice might seem unsustainable, but US universities have been relying upon such labor for 50 years.

Many, if not most, of those who have repeatedly not been hired or promoted to tenure do not leave academia, but become 'support staff' members, simultaneously serving as perennial exemplars of the 'up or out' disciplining and punishment. The more secure support positions include joining the university administration apparatus, becoming a researcher for large projects managed and led by others, joining a university press as an editor, or becoming a program officer at a research funding agency. Holders of support staff positions know that they can never be promoted to the senior levels of university administration which are reserved for tenured faculty members who have resolved to do full-time administrative work. Unlike the corporate world and more like the military and many religious organizations, universities are highly stratified systems in which people in some positions can never be promoted into others. All aspiring and current holders of faculty positions are surrounded by the consequences of not becoming the right kind of academic subject. The display is certainly not a necessary, but unfortunate byproduct of innovation or excellence, nor is it admirable, useful, productive, or sustainable.

\section{Becoming Academics in Norway - Knut's Account}

Formal systems of research training in Norway emerged while I was a graduate student and a post doc in the 1980s. Traditionally, university studies led to a cand. mag. degree (four years) which was the most common choice, and a Master's degree (at least two years, usually more) or professional degrees (four to six years). Taking a doctorate was often a life-long achievement, a culmination of your academic career. This happened rarely. Prior to 1980, there were no formal systems of coursework and supervision and no culture for mentoring of PhDs. The sentiment was clearly expressed by a prominent professor who stated that 'good students do not need supervision. If they need supervision, they're not good enough'. On the other hand, you could be 
hired as an assistant professor on a permanent basis. You might have benefitted from having a $\mathrm{PhD}$ to get a promotion or a raise, but promotion even to full professor - could be awarded based on publications without any requirement of a $\mathrm{PhD}$. Several outstanding academics signed a petition in the 1970s, stating that they would abstain from getting a doctorate because they considered such degrees a symbol of academic individualism that should be left behind. Later, some of them got a doctorate, acknowledging that the status was useful.

I entered a PhD program in 1981, working with a professor coming from the US, together with three other students. Or rather, we were not students we were formally denied that status by the student welfare organization but something else, a bit undefined. All four of us had Master's degrees in engineering or applied physics. Thus, our professor insisted on much more coursework than later became the norm, including one year of basic sociology, but we enjoyed extensive discussion about action research and industrial sociology. On the other hand, the professor showed little interest in my dissertation; I am not sure if he ever read any of it. I got a PhD in something termed 'organization and work life studies', which had an interdisciplinary ring to it. Thus, it was open to interpretation if I would qualify for a position within a social science discipline (I experienced that the interpretation could go both ways).

The development of $\mathrm{PhD}$ programs in Norway was a slow process that met with resistance. As a post doc beginning to supervise a couple of $\mathrm{PhD}$ students, I remember attending a meeting of potential supervisors where several of the elderly and established professors were critical of these new ideas. They deemed $\mathrm{PhD}$ programs unnecessary, maybe even as a system that might erode the individual autonomy of future academics. This meant that for a long time, Norwegian universities, including NTNU, struggled to establish a culture of supplying appropriate courses and effective supervision for PhDs. An intermediary step was to organize courses on a national level. I attended several. They were of high quality, often with a handful of eminent international scholars as speakers. After the mid-1990s, this changed, but still many PhD programs offer few courses and students may have to attend courses at other universities, also in other countries. This is useful in terms of networking and getting input, but it is troublesome with respect to having a shared base of knowledge. Today, as noticed in the previous chapter, Norway has adapted the so-called Bologna model, which means that a Master's degree is a prerequisite of entering a $\mathrm{PhD}$ program. $\mathrm{PhD}$ studies are expected to take three years; of this, coursework is supposed to take half a year. Therefore, the main part of the research training is supposed to take place through the work with the dissertation.

$\mathrm{PhD}$ students will have one main supervisor, sometimes with one or two co-supervisors. The options for choice are limited. Often, the department will appoint the supervisor - when PhDs are externally funded, which they often 
are, the main supervisor will usually be the project manager. It is possible for students to ask for a replacement - there are bureaucratic procedures for this - and if such inquiry is made, it is usually accepted. Such changes may be painful, because it involves criticism of previous supervision, sometimes personality mismatches. Some students try to get around the problem by including a co-supervisor that in reality becomes the main supervisor or by asking for informal supervision. I have been involved in both kinds of situations; their ghost-like features are not comfortable. Supervision is a critical feature because it is so central to the research training. In many situations, the supervisor(s) is/are the main intellectual resources available to $\mathrm{PhD}$ students. When asked about their experiences - such surveys are made regularly - the most common complaint is - unsurprisingly - with supervision. There is not enough of it, there is a lack of mentoring, and so on. While the official PhD regulations stipulate in some detail the obligations of supervisors, such as advising on methods, the use of theory, ethics, and networking, there is no established standard for how to meet the requirements. ${ }^{7}$ Often, supervision means mainly to provide critical feedback on written drafts, without much constructive advice about how to do the research and how to write about it.

During the last two decades, the dissertation format has changed. The monograph used to be dominant, above all outside the STEM disciplines, but it has increasingly been replaced by a collection of articles with a lengthy introduction that expands the theoretical and methodological considerations and summarizes findings. The latter format has also become common in the humanities. This means that $\mathrm{PhD}$ students are expected to write three to five publishable papers. However, often, in particular within the STEMM disciplines, the articles are co-authored with the supervisor(s) and possibly with other people that have contributed to the research. In this manner, most PhDs are part of teams. This collaborative model sometimes causes controversy regarding authorship, usually emerging from $\mathrm{PhD}$ students that are dissatisfied with the distribution of credit. Supervisors may also be unhappy with not being credited as co-authors of papers they have spent a lot of time and effort contributing to. Still, the model and the article format of the dissertation provide for academic subjects that are adapted to teamwork and the publication of journal articles in a way that prepares them for future academic life more effectively than the old focus on the single-authored monograph. The status of the single-authored monograph is, in my experience, declining considerably.

It is broadly acknowledged at NTNU as well as at other Norwegian universities that the quality of $\mathrm{PhD}$ supervision needs to be improved. The main strategy is to offer more training to supervisors and seminars to discuss challenges with supervision. The latter initiatives are interesting since, in my experience, supervision has been conducted according to supervisors' personal preferences and experience. An obvious issue is how supervisors are credited, in terms of workload. In many STEMM disciplines, supervision is 
considered as part of the research of faculty and not part of their teaching; thus, not included in their teaching load. In the humanities and social sciences, it has been calculated as part of the teaching duties, with 70 hours per year for three years to be shared by the supervisors. This is going to be reduced. The underlying expectation is that $\mathrm{PhD}$ supervision shall increasingly be integrated into the research conducted by faculty members in all disciplines. This reduces the options for PhDs to conduct independent research, while implicitly suggesting that supervisors need to plan that they also get publication opportunities from the collaboration. In the humanities and the social sciences, this is a considerable symbolic but also practical shift both in research and supervision practices.

The change from the monograph to the article-based format has transformed the research training, since writing articles raises new challenges regarding length, precision, and quality to fit the format required by most journals. The advantage is obvious, since articles increasingly have become the dominant form of scholarly publishing, also in the humanities. However, in my experience, this format is more demanding for students, who have to learn how to select journals, how to adapt to their different formal requirements, and not the least how to cope with reviews. As we all know, quite a few reviewers are pretty heavy-handed in their feedback, which is a challenge to upholding self-confidence. Thus, supervision ideally requires substantial exercise of care. This is not always offered.

Self-confidence may be a problem for many academics, since we invest a lot in our work, particularly publications, that may be subject to negative assessment from journal reviewers and colleagues. This is not the least a challenge for graduate students, who in addition may struggle with concerns for their future career, how and where to get a job, and so on. Sometimes, the feeling of inadequacy is self-diagnosed as the so-called imposter syndrome that Sharon also mentioned, a feeling that one is not deserving to be in academia, that achievements mainly owe to luck, and that it is just a matter of time before one's academic inabilities are revealed. This points to the importance of belonging to a supportive community; not everybody has access to this. I try to tell my students when they admit to such problems, maybe to their disappointment, that I have met quite a few that suffer from similar challenges. Actually, what they thought was a special problem is normal. I think 'normal' is good, as long as it is widely defined.

\section{Employment and Admission}

In Norway, nearly all $\mathrm{PhD}$ students are hired as research associates for three to four years with a minimum wage of around USD 57,000. They are formally considered as regular, if temporary, university employees, with health insurance and social rights, for example unemployment benefits and paid parental leave for 49 weeks after childbirth. With the hiring normally follows 
a small grant to cover research expenses, including travel. This means that the students have their own funding to travel to conferences; such participation including presentation of papers is expected as a part of their becoming PhDs. NTNU may offer additional funding and usually equip $\mathrm{PhD}$ students with computers and software as well as office space. There is no tuition. The employment format means that $\mathrm{PhD}$ students are recognized as regular staff members of their department, to be included in the everyday life matters. This means that they are supposed to be invited to development discussions with department leadership, to strategy meetings, and so on. In many departments, $\mathrm{PhD}$ students are a source of social renewal and diversity. The inclusion into departmental affairs should teach graduate students about decision making; in practice, about how department leadership makes decisions. However, this may appear rather opaque to many of them.

To be admitted to a $\mathrm{PhD}$ program usually requires funding that covers wages and social costs for a research associate as well as some overhead to the university. This means that one applies for $\mathrm{PhD}$ positions that are advertised, either with funding from the university or, more often, paid for by external grants that faculty members have acquired, usually from the Research Council of Norway. In the latter case, the dissertation topic is more or less defined by the project description. Thus, often, applicants are mainly asked to provide a letter of motivation and an outline of research interests, in addition to their $\mathrm{CV}$ and publications. However, in some cases, they are required also to outline their plans for their dissertation. Admission to the relevant $\mathrm{PhD}$ program is based on a research plan that the student must write when s/he is employed, but the real challenge is to succeed in getting a position, not to be admitted. The number of applicants to $\mathrm{PhD}$ positions vary a lot, which means that competition varies. An increasing number comes from other countries. In 2018, nearly 40 per cent of the graduated PhDs were non-Norwegian citizens. Some STEMM disciplines complain about a lack of applications from Norwegian citizens, explaining this as a wage issue. Especially, with an MSc in engineering or computer science, jobs in industry are much better paid than $\mathrm{PhD}$ positions.

\section{Transformations}

Before 1990, there were few positions as research associates at Norwegian universities. Since then, there has been a spectacular growth. In 1990, NTNU had around $130 \mathrm{PhD}$ students. In 2020, the number was 1720 . This reflects the relative increase of university research, compared to teaching. The introduction of $\mathrm{PhD}$ programs and the growing number of $\mathrm{PhD}$ students have also transformed the research culture and tilted university ecology toward junior academics. The latter change has been reinforced by the tenfold growth in the number of post-doctorals and researchers from 2000 to 2019. A simple indication of the growth of research at NTNU is the fivefold increase in 
publication output in the same period, as measured by Norway's system of publication metrics. ${ }^{8}$

The national emphasis on training more $\mathrm{PhDs}$ has introduced more research collaboration, since $\mathrm{PhD}$ students are expected to work with faculty members. Their dissertations form a very important part of present university research and are resources for faculty members. This has stimulated the development of research groups that often consist of one to two professors and two to four PhD students, sometimes also with a post doc or researcher. In the humanities and social sciences, such groups were rare before 2000 (but were more commonly found in STEMM research).

Thus, the emerging becoming processes of academics have also shaped the subject formation among faculty. The increased dependency on external funding requires a stronger entrepreneurial subjectivity. Faculty meet a growing pressure to acquire such funding, even if this is not yet a requirement in the Norwegian contexts. Faculty are paid for the whole year, including five weeks of vacation, independent of their success with research grants. The growing pressure is sometimes openly resisted. Some claim that external funding is not needed in the humanities and social sciences and that grant applications are too much a game of chance. Since the establishment of new fields of research, often interdisciplinary such as STS in Norway, depends on external funding, I find this view rather arrogant and selfish.

In parallel with the growth in the number of $\mathrm{PhD}$ students and the changes in the university research culture, a research bureaucracy has emerged. One of the main tasks is to survey the PhD programs, based on new sets of metrics. As already mentioned, the main focus in Norway is throughput (completion of the degree within the normative time), which is particularly emphasized by the government. PhDs are supposed to finalize their degrees when their funding ends, after three years (four years if they have an extra year to teach). Most of them spend more time, but considerable bureaucratic attention is given to reduce the average time spent on getting a $\mathrm{PhD}$. This focus on throughput, which is reinforced by a system with strict time limits on funding, implies a streamlining of the $\mathrm{PhD}$ process that confines the academic freedom of $\mathrm{PhD}$ students and their possibilities to explore alternative theoretical and methodological options. Clearly, it formats them as time-sensitive, punctual, academic subjects, appreciated in applied research.

As suggested above, dissertation topics are often predetermined by the external funding or strategic considerations of NTNU, and their choices regarding coursework are limited by the small number of relevant courses that are offered. This means that the academic freedom of $\mathrm{PhD}$ students is negotiated in a cultural setting that hails professional independence but provides inadequate resources for its pursuit. On the other hand, acquiring a doctoral degree in 'good old days' was based on 'the loneliness of long-distance running' and the mental strains of individualism. It was ... different. Individualism and independence are praised on ceremonial occasions, but in 
practice when hiring academics, a capacity for collaboration is increasingly emphasized.

\section{Getting Out of Limbo}

I sometimes say that PhD students occupy a liminal space. At least it is to some extent a protected niche that allows for professional development and learning about academic citizenship, even if it is intersected with meritocratic expectation and uncertainty about future possibilities. There is no welldefined initiation ritual; you get a letter of hiring by email and are expected just to turn up on your first day of employment. With respect to the ritual for incorporation, Norwegian universities use the ritual of the public dissertation defense, the disputation. However, there are distinct differences with respect to ceremonial details. After the dissertation is handed in, the faculty appoints a committee to assess it. The committee has three members. One internal, a departmental faculty member, and two externals. In most cases, the assessment concludes with the phrase: the dissertation is accepted for defense. In practice, this means that the student has passed.

The dissertation defense is a public ritual where the student must present the thesis and defend it from oral critique from the two external members of the assessment committee. Commonly, at least 20 to 30 people are present, including colleagues, friends, and family. The dean or a representative of the dean chairs the event, robed in a traditional academic costume. Afterwards, there is usually a party to celebrate the conclusion of the $\mathrm{PhD}$, with speeches applauding the newborn doctor. Many students are nervous about the occasion and unsure about how they will perform in front of friends and family. They may have been present at colleagues' defenses, some of which went well, some where the student struggled with responding to the critical inquiries to an invited outsider called the opponent. Mentoring usually helps, but nerves are still present, since the defense is a public performance.

Ideally, the dissertation defense is an opportunity for dialogue, where the opponents and the student together explore strengths and weaknesses of the dissertation to discuss possible future steps and additional interpretation of findings. When this is not happening, it may be due to opponents who see the defense mainly as an examination to disclose weaknesses or who are overly critical. Some try to enact the classic disputation, which was a kind of academic infighting. We always have at least one opponent from a university outside Norway; sometimes, we observe in play academic traditions where critique is appreciated more than constructive interventions.

It is debatable if the dissertation defense really leads to incorporation into academic society in Norway. The $\mathrm{PhD}$ has become a mandatory step in an academic career, but the degree has lost its former status and competition for faculty positions has grown. Traditionally, getting the degree was a high point, but increasingly it is a box to tick. Formally, in Norway the PhD qualifies for 
a position as associate professor, but the road to such positions is long and winding. Nothing has changed, but now there seems to be greater concern than there used to be. The competition has grown also because there are more people from abroad that apply for faculty positions in Norway. Some of them are what we may call traveling post docs, academics that for a long time have moved between countries and post-doctoral positions to acquire impressive publication merits.

\section{Assessment Experiences}

Becoming an academic means to be formed as a review-ready subject. When applying for academic positions - I have done my share of that - you prepare for being evaluated by a committee of people supposed to be peers (they are not always). This is risky, since you may be severely criticized and/ or deemed irrelevant like I have on several occasions. For example, once a professor of business administration characterized me as a philosopher, meant to disqualify me for a position that someone else was supposed to get. Such assessments are painful; becoming an academic may train you in pain management, but also teach you to cope with anger, frustration, denial, and maybe also shame. The latter feeling inhibits the sharing of the experience with friends and colleagues, which is unfortunate since such sharing often provides healing. Applying for academic positions means in the Norwegian context today to provide a $\mathrm{CV}$, selected publications - preferably with an overview of their content and framing - and a teaching portfolio. Teaching experience and resourcefulness is increasingly emphasized. The appointed evaluation committee will usually rank three to four people on top; they will be invited to do a trial lecture and an interview. The number of applicants fluctuates, but sometimes there are not more than ten. The level of competition in Norwegian universities varies a lot.

The trial lecture and interview will be conducted by a committee of faculty members at the department - usually three to four - and two student representatives. They will rank the candidates in a report sent to the head of the department, who may or may not change the ranking, before the documents are sent to the faculty. The final decision is made by the board of the faculty after the hiring has been discussed in the hiring board, where unions participate. Many faculty members have joined trade unions. In general, only a few faculty members participate in such hiring processes, and there is rarely much collegial debate about hirings. I have experienced only one or two occasions where some of us have protested the outcome. Applicants may protest but do so seldomly, because protests can only be made with respect to formalities, not regarding professional assessments of quality and performances.

My own experience in applying for positions may demonstrate another kind of process, which is uncommon but not rare. In 1988, I was asked to lead a new STS center together with a friend and colleague. We had been active in 
trying to establish the center. The positions were not advertised, but to avoid formalities, we were engaged in temporary positions with an independent research institute affiliated with the university. Five years later I was asked to provide the necessary documents to be assessed by a committee, potentially as professor of sociology of technology. I was found qualified, hired, and formally became a member of faculty at the Department of sociology without further consideration, while continuing my work at the STS center. Some years later, NTNU's rector gave us the choice between merging with a center for women's studies into a new department or being discontinued. The choice was easy. Suddenly I was a professor of STS in a new department placed in the Faculty of Humanities.

I have never been exposed to a merit review; NTNU does not apply such practices. After being hired in a permanent faculty positions you may only be reviewed in the context of wage negotiations where trade union representatives participate; you may be asked to provide a brief form with simple metrics about publications, grants, and supervision during the last few years. However, often, other considerations lie behind raises; you will never know why you got a raise or not.

The STS group I work with has been evaluated twice during its 30 years of existence. These reports have never assessed individual achievements, only the collective performance. As an associate professor, the only performance review you encounter is when you apply for promotion. Promotions in such cases are decided in the same way as hirings; there is a committee of three peers that provides an assessment report. Their decision is normally just rubberstamped by department and faculty leadership. Other faculty members are not involved in the decision. However, when people apply for promotion, they may be advised to do so by senior colleagues.

Some departments use so-called appraisal interviews done by the head of department or another member of departmental leadership as an occasion for assessment of performance. Normally, this is a push for improvements with little opportunity for leadership to reward or punish. As previously noted, there are not many resources distributed by departmental leadership, so there is little to give or take away. However, there are institutions where the share of working hours allotted for research is negotiable and where faculty members who are considered underperforming, may be required to do more teaching - thus given less time for research. Some departments also reward financially prominent publishing achievements, but most departments lack funding to do so. Appraisal interviews have a recommended format but is usually not based on form-filling.

To some extent, Norwegian academics is protected by a work-life culture with considerable legal regulation of the work environment and trade union representatives that may interfere in situations with unfair treatment. Maybe the sparsity of merit reviews reflects this work-life culture, where managers tend to be reluctant to criticize their employees openly and explicitly. This 
does not mean that Norwegian universities are free of bullying. Harassment is potentially present among colleagues, as noted in the previous chapter, and it tends to be overlooked by being characterized as 'just' professional disagreements. The formation of academic subjects may fail to shape academic citizenship to perform civility.

The absence of systematic individual performance reviews does not absolve Norwegian academics from neoliberal subject formation. We are surrounded by authoritative voices that tell us that we need to perform better. I definitively see myself as formed to become an entrepreneurial academic. Since I started my academic career, I have initiated or been part of at least one grant application every year, often three or four. I have been engaged in establishing and expanding a department, trained a substantial number of PhDs, done my share of mentoring and publishing, and been active in university politics. Does that make me an 'ideal academic'? Such discursive constructs abound, but they tend to sidestep the fact that the imagined scientist of policy makers has many deficits (Åm et al., 2021). Given the reforming zeal of the Norwegian government that we described in Chapter 2 and the comprehensive set of goals NTNU faculty are supposed to pursue, the ideal academic would have to be a superhuman with multiple identities. Nevertheless, there is governmentality at work that forms us as academic subjects, but in the plural. To provide the public goods that the Norwegian government asks for, such plurality is needed. We all do our bits and pieces, making our priorities as academic autonomy allows, and hoping it adds up as an acceptable collective performance. In this Norwegian context, a singular quest for excellence does not work that well, which I think is a good thing.

\section{Systems of Formation of Academic Subjects}

When we juxtapose our observations and experiences, this invites the question: Do UCLA and similar US universities form academic subjects differently from and with better resources than those formed at NTNU and other Norwegian universities? On one level, the answer seems to be yes, given that UCLA scores much better on international university rankings than NTNU. Thus, presumably, UCLA faculty members perform better than NTNU faculty. UCLA's emphasis on performance metrics and the application of such metrics in individual merit reviews seems to pay off in the quest for excellence in university rankings. However, this judgment may rest on an ecological fallacy - concluding from the collective to the individual level. A more important question is to what extent the formation of academic subjects is fundamentally different in the two contexts. The answer depends on what we see as the main features defining academic subjects. To what extent are these features given by university rankings and the related quest for excellence?

McAlphine and Amundsen (2018) show how diverse young academic subjects may be. Such diversities are in line with our own experiences that 
tend to contradict ideas about a single ideal academic subject, even in a given context. When we juxtapose our two narratives, we see that the academic subject formation in both cases produces entrepreneurial agency. To some extent, such agency has always been asked for, but the neoliberal context of universities for the last 30-40 years has intensified expectations of academics and made becoming processes more demanding, constrained, and risky. The authoritative ideas about what are the right things to do have increasingly been shaped by the growing arrays of metrics that are supposed to measure academic performances; what cannot be measured does not count.

Sharon has described the paradox of corporate entrepreneurship in academia, a prolonged double-bind performance: productivity can only be maximized by rapidly generating more of the same kind of research and teaching, hopefully of some longstanding significance, while actively engaged in the everyday admission, evaluation, and budgeting processes according to the corporate algorithmic governance apparatus. Innovation is generated by pursuing new lines of inquiry that require large investments for not only building the ideas, but also building academic scaffolding around the ideas so that they can circulate, attract interest, become useful to others, and survive. Doing both kinds of tasks well is nearly impossible, but required; that leads to the double bind: doing one well means doing the other far less well. The stress of trying to do both can lead to either a very troubled subject or an increasingly shrewd performance of bad faith. Marx might have seen those conditions produce alienation from academic work.

In an ironic way, the formation of academic subjects at NTNU is softer and more diversified than at UCLA due to the reforming zeal of the Norwegian government and the extended requirements for public goods. As noted above, NTNU must provide for more academic plurality to meet these requirements. UCLA, which operates within a political economy that makes the university financially dependent upon its reputation and position in university rankings, pursues a more singular quest for excellence. In turn, this leads to a more unambiguous formation of academic subjects according to excellence metrics. The announced goals are for innovation and originality, but the focus at every stage on productivity metrics makes clear that the goal is quantity.

We certainly will not rank the two systems; we leave the ranking enterprise to others. Our main point is to demonstrate and elaborate on the differences and similarities. Academic life is nevertheless challenging, and the formation of academic subjects is a disciplining process that may be experienced as more or less tolerable. It involves not only engaging with what is expected of us as graduate students and faculty members at different career stages, but also how we learn to alter the ecologies in which we are embedded, such as through hiring colleagues, and also how we adapt to the changes in our local ecologies wrought by other forces. Outsiders may survive, even succeed, since assessments at the end of the day are contingent and somewhat unpredictable. Felt and Fochler (2012) introduce the concept of epistemic living spaces 
to describe the conditions of academic work and how neoliberal reforms have tended to constrain these spaces and make them more difficult to bear. Precarity as a growing phenomenon of universities in many countries exemplifies some of the potentially dire consequences. It has emerged through growing financial strains due to cuts in public budgets. Precarity seems to contradict the belief in universities as critical institutions in the development of so-called knowledge societies. In the next chapter, we address this belief by investigating how universities interact with society through teaching, innovation, and interdisciplinarity. How may this explain the central position of universities in knowledge societies?

\section{Notes}

1 For UCLA data see the Admission, Enrollment and Degree Data by Graduate Program www.gdnet.ucla.edu/asis/progprofile/profile.asp

2 www.osb.org/gen/rule.html

3 See also Clark (2008) and Becker and Clark (2001). Memoirs of professional education show many parallels with military recruit training in 'boot camp'. See Henry (1983), LeBaron (1981), Turow (1977), and White (1991).

4 www.apo.ucla.edu/academic-listings/endowed-chairs

5 An example is the University of California Academic Personnel Manual www. ucop.edu/academic-personnel-programs/academic-personnel-policy/

6 Dubinskas and Traweek (1984).

7 https://lovdata.no/dokument/SF/forskrift/2018-12-05-1878

8 https://dbh.nsd.uib.no/statistikk/rapport.action?visningId=278\&visKode=false $\&$ admdebug $=$ false $\&$ columns $=$ arstall $\&$ index $=1 \&$ formel $=1125 ! 8 ! 1126 ! 8$ ! 1127 ! 8 !1128\&hier=instkode!9!fakkode!9!ufakkode!9!itar_id\&sti=\&param=arstall\%3 D2020!9!kanaltypekode\%3D1!8!2 


\section{The Meshworking University \\ The Traffic in Knowledge through Teaching, Innovation, and Interdisciplinarity Practices}

\section{Introduction}

In standard accounts of university history, there are two major transformations. First is the emergence after 1770 of the so-called research university, which according to Clark (2004) developed in response to market forces and government bureaucracies, producing a new kind of academic whose goal was to do original work and achieve recognition through publication. Second, the advent of 'the mass university' came after 1960, with a huge increase in the number of students and faculty members. There was a parallel rise in university research, reinforced by increasing investments in research, both public and private. The result has been, as we suggested in Chapter 1, that universities today are seen as a critical part of modern society, far beyond the moderate expectations of the past.

However, as we have noticed, in some countries this view has been moderated by economic setbacks and the introduction of austerity measures in public spending. Notwithstanding those obstacles, OECD continues pushing for greater investments in research and innovation, and its latest report on precarity is seriously concerned about the future academic workforce (OECD, 2021). The EU stresses the importance of research and higher education, ${ }^{1}$ and the US under President Biden will increase its spending. ${ }^{2}$ According to a report from the European University Association, most European countries provided growing funding to universities from 2008 to 2019. The growth in investments were largest in Austria, Belgium (Flanders), Germany, and Norway. Decline is observed in Italy, Spain, and the UK. ${ }^{3}$

Given those investments, universities have become objects of much political attention and increased expectations regarding the public and private goods that they are supposed to provide and the efficiency through which this shall be accomplished (Gumport, 2019). We described this in Chapter 2 while noting the considerable difference between NTNU with a clearly present Norwegian government and UCLA with a far more absent government. What we see in the European, including Norwegian, context is how policy makers 
want the provision of public goods to accelerate; universities are supposed to help solve the pressing grand social challenges, such as climate mitigation, food scarcity, poverty, and health inequities. The Covid-19 pandemic and the subsequent chase for vaccines and cures is a case in point. The expectation of such public goods from both NTNU and UCLA is compounded by the presumption that they also will provide innovations, spin-offs, and useful engagements with a wide array of agents from different sectors of society, as well as enhancing the position of its graduates in demanding labor markets.

There are three main neoliberal strategies to make universities accelerate their provision of those public and private goods. The first is an extension of the expectations. Traditionally, universities have had two main tasks - teaching and research - supplying society with educated adults and a storehouse of knowledge. Now, they are expected to supply industry and the public sector with accessible knowledge that can be transformed to innovations, both social and technological. Moreover, university graduates should have acquired the kinds of knowledge that meet the current needs of employers. The second strategy is financial, making university funding increasingly dependent on the production of the required goods, and that productivity should be measured meticulously in order to improve efficiencies. Third, neoliberal policies call for improved openness and transparency of universities; in practice this means that industry, government, and the public should have easier and faster access to the knowledge that universities produce, synthesize, and store. The underlying imaginary is that such access will enable increased innovation and economic growth.

There are many faults in this acceleration imaginary, along with the putative solutions to problems that do not exist. To begin, there have been many criticisms of the linear model that basic research on fundamental processes leads directly to useful, innovative ideas for addressing current social needs. Moreover, the arguments of the so-called Plan S imply that industry and the public sector should have immediate access to newly published scholarly articles. ${ }^{4}$ This ignores the critical role of universities in assessing the quality and relevance of that new knowledge and in synthesizing the new findings. (Of course, open access publishing of scholarly research clearly is useful for universities everywhere that cannot afford the high cost of many journal subscriptions.) Acceleration policies imply that universities have erected unnecessary barriers to their accumulating knowledge, restraining innovation. The imaginary also underestimates universities' current engagements with wider society and misunderstands the mechanisms and channels of such engagements.

That acceleration imaginary may be seen as a response to another faulty stereotype. Conventionally, a university is represented as a place, a specific space in which the work of academia is conducted. In the US that place typically is called a campus, a concept increasingly used in Norway too. The stereotype is that it is both a park-like environment and an 'ivory tower' or a 
distinctive 'city on a hill' apart from the work of commerce and government. However, in fact such places rarely have been self-contained; people work and study in universities, but most of them live elsewhere. The porous border of the campus has raised problems of jurisdiction for centuries, as represented in the old 'town-gown' binary. Meanwhile, the towns around universities have long prospered from all the traffic into and from universities. About a century ago 'real estate' entrepreneurs in California realized that hosting a new campus of the University of California system would increase the price of the neighboring land, so they have donated land for the new campuses; UCLA is but one example. The Janss Steps, a UCLA landmark, was named after such entrepreneurs. ${ }^{5}$ They also engaged in racially discriminatory housing practices, and there are recent demands to rename the long outdoor staircase after the Tongva indigenous people whose unceded lands the university occupies. ${ }^{6}$

During the last 50 years US universities increasingly have become venues, eventful spaces where sports, concerts, and other cultural activities are held. At UCLA those public events have become an important 'revenue stream' with university offices managing the spaces that can be used, charging the fees, and making the arrangements for services like food and parking. ${ }^{7}$ The campus is seen as a desirable location for many kinds of events, including filmmaking, which in turn increase those who want to use the space. When UCLA faculty members want to host academic events on campus, we too must make reservations and pay fees from our grants. About 15 years ago Sharon learned that UC Irvine, about an hour from UCLA, charged far lower fees and began planning events at UCI for that reason. During the pandemic the loss of event fees became worrisome at UCLA, identified as one of the five major sources of income for the campus, along with student fees, donor contributions, research grants, and other services. NTNU's campuses are less eventful, although they serve as conference locations and a meeting place for many knowledge society actors.

Thus, from our perspective in this book, the university is now best seen as a shared space, a crossroads, a trading zone, a borderland, and a commons, where the traffic in ideas circulates and knowledge grows. The daily agents of that circulation include the students, alumni, faculty members, administrators, and a constant stream of visitors. Calls to make universities 'relevant' to society and to make university knowledge accessible to the public rely on the image of the university as isolated, self-referential places that might be suitable sites for cultural events, but not as busy crossroads which they increasingly have become over the last 50 years. Shapin (2012) has analyzed critically the emergence of the ivory tower stereotype, showing that in fact 21st-century scientists are deeply engaged in socially relevant issues. Universities are not and have never been ivory towers; they are too deeply engaged in traffic in knowledge, in making knowledge mobile, and in complex exchanges with a multitude of actors. 
As demonstrated in earlier chapters, many universities are filed with very competitive entrepreneurial academics and the relationship between universities can be at least as competitive as any other sector in society. Åm et al. (2021) found that Norwegian science policy makers widely employed a discursive construct, an imaginary scientist, who were insufficiently engaged with social problems - an indication that the 'ivory tower' remains present in research policy. The scientists that Åm and collaborators interviewed countered this view by emphasizing that social utility was a strong motivating factor of their research.

From a neoliberal perspective, the ideal is 'the service university' (Cummings, 1998) that 'develops products that are competitive in a knowledge market' (Tjeldvold, 1998: 9). Thus, what comes to mind as the neoliberal alternative to the ivory tower is the metaphor of the shopping mall university, where students and other actors may select knowledge products from the shelves of a variety of department stores. As we saw earlier, many students now use universities exactly that way, selecting an assortment of epistemic resources as important tools to accomplish their goals, unconfined by the traditional narrow curricular requirements. The European University Association published a vision document in 2021, aptly titled 'Universities without walls', proposing another metaphor to replace the ivory tower. Their metaphor allows diverse interpretations; one possibility is to think that society largely will become an academia: through increased openness and engagement with social problems universities will be embedded in society as a whole, much as STS laboratory studies have argued. Thus, a growing part of human practices will be guided by knowledge making, drawing upon scholarly research. While this is an interesting vision, it probably overstates the epistemic power of universities. It seems particularly indebted to older ideas of technocracies, from Saint Simon to Daniel Bell, that technocratic elites are best able to judge what societies need and govern accordingly, a model particularly attractive to powerful bureaucracies around the world since the 17th century, at least. Another, perhaps more favorable interpretation is the idea of universities as knowledge commons with inclusive policies regarding who may contribute and who may harvest.

Having asserted that the neoliberal model is based on a set of clearly false assumptions, we could ask why that model has persisted and gained as much influence as it clearly has. If its assumptions are so readily refuted, why has that been ignored? That silence and apparent bad faith point to ideological practices and motives, which we address in the concluding chapter. In this chapter, we explain how NTNU and UCLA already do, in fact, engage with society in ways that the neoliberal models, including the technocratic version, do not and cannot grasp. Here, we draw on an image of our universities as knowledge commons. They make, assess, synthesize, store, and distribute knowledge widely. Commons have been observed to be threatened by overuse and depletion of resources. Such 'tragedy of the commons' (Hardin, 1968) 
does not affect knowledge commons. They cannot be overused or depleted but are rather strengthened by being frequently accessed and used. Knowledge flows to and from universities in complex ways, sometimes orchestrated by faculty members, sometimes driven by particular searches from other actors.

Thus, we may understand universities as a nexus between research and society, defining NTNU and UCLA's engagement with society as meshworking. We use this concept to emphasize the interactive and rhizomic features of three ways in which our universities already are complexly engaged with society: education, innovation, and interdisciplinarity. In this manner, we provide a different perspective on universities' quest for excellence. As we have noted, research excellence may be counted upon as a source of reputation, as well as epistemic authority. However, with more pragmatic considerations regarding the public and private goods expected from universities, excellence may need to be considered in plural. In a world where it mainly is metrics that count, it is tempting to think that excellences may be aggregated by simply adding them. We demonstrate why policies based on additive thinking may hamper the traffic in knowledge because it overlooks the faultlines between the areas where excellence is assessed.

This chapter continues with a critical investigation of the slogan 'researchbased teaching' and the growing emphasis on teaching quality as a concern separated from research. On the one hand, we argue that teaching is a vital channel for selecting, integrating, and making relevant research that is currently available. On the other hand, we warn against what we see as an emerging faultline between teaching and research, which may make teaching a less effective channel of bringing research into wider society. We also are critical of the predominance of one-way (linear) thinking about the relationships between universities and society in standard discourses of research circulation and university-industry relations, usually discussed in terms of diffusion and impacts. To go beyond this, we explore these two relationships of teaching/research and universities/society by analyzing how universities are public spaces that co-morph these knowledge relationships largely through teaching and faculty members' exchanges with colleagues in other sectors. The traffic in knowledge is embodied: former students and faculty members circulate widely.

Our alternative is to see universities as contributing to growing, meshworked knowledge commons where much knowledge is liminal with respect to its use; that is, the relationship between knowledges and their uses is necessarily and fruitfully ambiguous at certain stages and should not be prematurely determined and classified. We show how liminality may result in knowledge mixing that mobilizes resources from the commons. That mixing should be encouraged, not quickly sorted, labeled, and measured. Furthermore, knowledge mixing is important for innovations. Interdisciplinarity is a significant part of those processes, found at both NTNU and UCLA. We demonstrate clear differences with respect to their institutional strategies 
and interdisciplinary practices for addressing the disciplinary faultlines, the interfaces, folds, and ruptures where much knowledge mixing occurs. As we have shown in earlier chapters, both NTNU and UCLA routinely domesticate the global demands for innovation and relevance. However, faculty members are left with the task of meeting those demands. Often that happens through their engagements with inter-, multi-, and transdisciplinarity.

\section{Transmitting and Transforming Research Through Teaching}

Conventionally universities are characterized as providing research-based teaching. This was inspired by Wilhelm von Humboldt's proposal for university reform from the early 19th century. He argued that universities should be institutions of research as well as higher education and that research and learning be unified. Students should be taught the knowledge generated by research and ideally how to generate new knowledge through research. Students were to learn about academic debates and the adjudication of quality in ideas. However, the concept of research-based teaching is notoriously unclear. According to our experiences, it is rarely discussed. Probably, it is implicitly understood as a rationale of university teaching. In their review of the large literature addressing the topic, Elken and Wollscheid (2016) find considerable diversity in definition and approaches. Many contributions are primarily concerned with the academic socialization of students and the role of students' participation in research as a pedagogical strategy.

We want to highlight that university teaching is a practice where knowledge is presented, assessed, synthesized, transformed, exchanged, and imparted. Thus, teaching is a comprehensive infrastructure where research - old and new - is made available to society through the graduates that populate an increasing number of positions in a growing variety of social fields. This means that university teaching is the main mechanism of making research useful and usable to society. It is the practice where students learn both how research is conducted and how to acquire knowledge from research - new and old - enabling them to bring this knowledge with them in their future roles as professionals, citizens, and perhaps as academics. Students are the carriers of the knowledge base from universities into society, far more important conduits than articles and research reports. Some companies even explain that hiring recent graduates is their main strategy to bring new knowledge into their organization (Hojem 2012). Alumni may continue to stay in touch with their teachers as part of the exchange of knowledge. At NTNU, the students' association every other year organizes a three-week long festival, which serves as a national meshworking event where former and current students, as well as faculty members, interact, not only in the evenings but during seminars adjunct to the festival. 
As we all are keenly aware, in any subject there is much more knowledge available than is humanly possible to learn. Universities are huge repositories of old and new knowledge; chances are great that much knowledge remains liminal, forgotten, or overlooked unless it is transmitted through teaching, not just as content, but also as analysis of the processes by which the knowledge was made. We want to emphasize first that teaching is a mechanism for making research publicly available; second, it is a resource for research, because teaching requires a lot of effort to select and synthesize research findings. That process challenges the teacher to articulate the research process but it also serves as a preparation for doing research, for example reviewing what presently is known. Moreover, students increasingly learn about conducting research and begin to do so. In such ways, teaching has become the most important channel of bringing research into the public domain. Unfortunately, this usually is not acknowledged.

By contrast, research policy tends to sidestep higher education by focusing singularly on direct dissemination of research, reflecting the prevalence of linear thinking. They do this by highlighting published papers as the main outcome of research and calling for commercialization and dissemination beyond teaching of such products. Those policies effectively frame universities as two bifurcated worlds: teaching and research, separating measures to improve teaching from policies aimed to make university research more socially useful. However, the policies reflect the lack of engagement with the relationship between research and teaching. What are the consequences of the bifurcation, and how do faculty members deal with the situation?

To the extent policy documents at our universities and at the national level mention the issue of research-based teaching, it tends to be understood mainly as an issue of the competence of university faculty: faculty should have documented competence in research in the fields relevant to their teaching. In a few places in the documents, faculty are required to learn about teaching, but there are few or no guidelines about evidence-based best practice to unify research and teaching. It is interesting to notice that the Standards and Guidelines for Quality Assurance in the European Higher Education Area, ${ }^{8}$ issued by the European Quality Assurance Register for Higher Education (EQAR), takes for granted that research and teaching is linked. The monitoring of teaching quality is supposed to include an assessment of 'The content of the program in the light of the latest research in the given discipline thus ensuring that the program is up to date' (Standards, 2015: 15).

A study conducted at NTNU analyzed how faculty navigated this situation and how they related research to teaching. ${ }^{9}$ To begin with, when asked about the meaning of research-based teaching, it was clear that they had not given much thought to the concept. Accordingly, their responses varied considerably. Some said it was about teachers having done research or about using their own research in teaching. Others explained it as using updated research as the basis of teaching or teaching students about research. However, for all 
of them research was a crucial resource, even if they used it in diverse ways. To clarify this diversity, the interviewees were asked about how they selected readings for their courses. The responses showed that there was no common strategy or criteria for selecting readings and there seemed to be little reflection about the selection process, although some mentioned considerations of the didactic qualities of readings and the need for updated material. Thus, the selection of course material appeared as a pragmatic matter. Most academics probably recognize the choices: textbooks, compendia, and published papers, including review papers. The texts could be written by the lecturer, colleagues at the department, or scientists/scholars nationally and internationally. Many also mentioned that they included research reports, computer programs, and internet resources to the extent they were considered appropriate to the course. Given the need to limit the volume of readings, the course material is highly selective, decided according to the learning goals of the course. By implication, a lot of available research was not selected.

A striking finding from the interviews was the importance attached to what we will call 'relevance work'. All the interviewees emphasized that their teaching should be relevant for the students and their future employers. The relevance work drew on one or more of the following resources:

- The experience and wider engagement of the lecturer.

- Readings from practice areas.

- Practice-oriented assignments.

- Guest lecturers from industry and public administration.

- Research engagement with industry and public administration.

Thus, in their teaching, the interviewed professors explained that when they presented research in their teaching, it was made sense of and amalgamated with knowledge related to the areas of future employment of the students. They explained that the relationship between research and teaching was rhizomic, with complex flows of knowledge and concerns.

That study confirms our claims above that universities are relevancemaking devices that translate research into something potentially applicable in the context of teaching. University teaching mobilizes research to prepare students for future engagements in society, including the ability of critical assessments. University professors mediate research above all by selecting reading material and interpreting this material by drawing on a broader set of research, often their own, and by presenting illuminating examples. They also integrate into their teaching experiential knowledge of conducting research but also from their extracurricular activities. In this way, they try to help their students to understand the practical implications of the research that is presented. Assignments are also used for such purposes. Another important observation is that the use of research in teaching is shaped mainly by considerations about what students should know to be able to do the tasks they 
might encounter in the future, either as advanced students or employees, and to be educated according to disciplinary or professional standards. That is, their teaching is not determined by their research, but it is a significant part of their teaching.

As noticed, there is little public reflection about what is and should be involved in considering university teaching as research based. Teaching is often described in ways that render these practices as linear processes of dissemination, with little concern about universities as meeting places where faculty interact with people coming from industry, the public sector, NGOs, professional unions, community activists, and, of course, the public. Such encounters may be face-to-face or mediated, on campus or elsewhere, and they are extensive and multi-layered. For example, professional associations are one of the arenas where university faculty historically have interacted with practitioners, exercising considerable influence with respect to the training of medical doctors, engineers, policy makers, and so on (Johnson, 1972). Another widespread practice is the use of people from industry or public sectors as adjunct faculty and guest lecturers. Work placements are common in the training of professionals; it is becoming more common also in disciplinary programs. For example, the Faculty of Humanities at NTNU offers a subject where work placement is an integral part. ${ }^{10}$ Many students also write their theses in collaboration with companies or institutions. Kristensen (2020), who has mapped how work-life experience is integrated in the education offered at NTNU, shows that such integration is frequent across all faculties.

At UCLA it has become common for departments to host events for undergraduates to hear presentations by people who had gotten a degree in the discipline. The point is for the alumni to convey to students how they were able to build careers in an array of fields. For example, the undergraduates in the humanities are taught they should tell future employers that they have learned an important conceptual strategy applicable in many lines of work: how to examine many disparate kinds of cultural materials carefully, think analytically about those materials, and find patterns among them. However, they normally do not learn how to present themselves as having specific cognitive resources or knowing how to make new knowledge.

There is a growing concern among Norwegian politicians that university graduates are insufficiently well prepared for their future participation in the labor market. The catchword is 'work life relevance' of study programs. The political concern has been partly fueled by an assessment study that argued that some university departments offering discipline-based programs did too little to integrate concerns about future employment of their students. The work-life relevance of these programs was deemed to be unsatisfactory (NOKUT 2020). We may discuss what work life relevance should mean and if the assessment was fair. However, the important point is that the resulting deficit understanding was quickly shared widely by Norwegian politicians 
who at the outset are skeptical of universities' ability to make knowledge flow effectively to external communities. NTNU and other universities do not quite understand their role as public spaces and are unable to document the extensive meshworks of faculty members, which make them unable to counter the deficit approaches of politicians and other critical, external actors.

\section{Regulating Quality}

Another challenge to the appreciation of research-based teaching emerges from the framing and management of quality issues. In Chapter 3, when we discussed academic citizenship, we emphasized how teaching quality has become an increasing concern and rightfully so. At NTNU, this is occasionally explained with reference to the growing number of students; the fact that relatively more young people study at universities is used to argue that there should be more focus on didactical issues. In this context, some consider the quest for research excellence a problem. First, research is seen as taking time and attention away from teaching. Second, it is assumed that faculty will prioritize research because it is presumed to be more prestigious than teaching. For example, as we noticed in the previous chapter, traditionally, hiring and promotion have been decided mainly on the basis of research achievements; teaching has been more of a box to tick. Faculty members were expected to have broad teaching experience but did not have to show any documentation of the quality of their teaching. As we have seen, this has been changing over the last 25 years. First, promotions now include more emphasis on teaching and teaching knowledge is highlighted in hiring processes. Second, efforts are made to increase the prestige of teaching, for example by promoting teaching quality awards. Norwegian universities have also introduced a new academic title that faculty may be awarded if they apply, based on a thorough examination of their teaching records and teaching philosophy. They become socalled merited teachers, a title that is an add-on to their position as associate or full professor. It may also give a wage rise.

Furthermore, there is a growing requirement that faculty at NTNU and other Norwegian universities take courses in university pedagogics. There are no such requirements in US universities, but increasingly graduate students who serve as teaching assistants are required to take such classes. Thus, there has emerged a clear generational divide in the professoriate separating those who have had such instruction. Also, the norms of what constitutes appropriate didactics have changed, not the least by highlighting so-called 'student active' or 'interactive teaching' methods. Centers have been established to support more experimental teaching methods, and universities allocate funding for such initiatives. These reforms are timely; the traditional university teaching with monolog-like lectures as the dominant method was clearly deficient. Both in the US and in Norway, that information often is conveyed in 'learning modules' teaching faculty members how to use the 
latest developments in the online 'learning management system' (LMS) that the university has purchased.

However, this emphasis on teaching has meant that didactics is prioritized over content. The discourses about good pedagogical methods are shared across disciplines and professions, which means that they engage little with content. Guidelines for assessing teaching quality often show this. The abovementioned report from EQAR states in one of the three instances where content is mentioned that

Quality, whilst not easy to define, is mainly a result of the interaction between teachers, students and the institutional learning environment. Quality assurance should ensure a learning environment in which the content of programs, learning opportunities, and facilities are fit for purpose.

(p. 7) $)^{11}$

The only thing the report says about quality of content is that teaching programs should be up to date.

Of course, it must be acknowledged that many teachers design their course plans in a formulaic way, covering the same topics in the same way they had experienced as undergraduates, while they might update the readings periodically. Sharon served on a departmental 'teaching committee' in which faculty members visited each other's classes and offered suggestions about pedagogy. Eager to see how one highly regarded teacher conducted his classes, she was startled to see a familiar syllabus. Later she compared it to the one she had been assigned in a class on the same topic at Berkeley decades earlier and found they were nearly the same. Many faculty members see undergraduates as consumers of foundational subject matter and students in such classes learn to be adept consumers; they usually do not learn either about the research process or about becoming knowledge makers.

The Norwegian government has issued a White Paper addressing pedagogy, called 'Culture for quality in higher education' (Meld. St 16 (2016-2017)). It notes that quality is a multifaceted concept but defines it as 'Quality implies to set ambitious goals and working continuously and good to achieve these goals .... Students shall

- Achieve the best possible learning outcomes and personal development.

- Encounter relevant education programs that prepare them well for active participation in a democratic and diverse society and for a future career.

- Carry though their education in the most efficient way (p. 15).'

In the conclusion, the White Paper refers to the existing education-related metrics as the main tools of governance. The governance parameters are quite general (see Chapter 2 for further discussion) and although there are institutional arrangements in place that require that all universities have systems for 
quality control of teaching, it is left to the universities, in practice to faculty, to translate the abstract and general aims into teaching methods and course content. This is in line with the emphasis put on academic freedom in other circumstances.

However, the governance parameters, the metrics, and the bureaucratic policing of quality control systems drives the bureaucratization of higher education. As we pointed out in Chapter 3, at NTNU, this leads to considerable work to produce the 'quality reports' that the system requires for each course and each study program. In turn, these reports are aggregated and in principle assessed by the university's teaching administration. Many faculty members complain about the time spent on reports; of course, some have found ways to game the reporting requirements. The bureaucratization of teaching evaluation is also evident from the introduction of learning management systems that digitalize and standardize the interaction between faculty and students. NTNU uses Blackboard as such an interface, together with Insperia, a digital exam system. As discussed in the last chapter, UCLA has been using Moodle, but switched to Canvas in the summer of 2021, changing from an LMS designed by a loose meshwork of university-based researchers in education technologies to a corporate model. In the US the largest corporations have long had their 'in-house' instruction systems and have systematized the apparatus of learning modules, assessments, and so on.

The formal evaluation of courses by students has been systematic in US universities for decades. Initiated in the 1960s and 1970s by students, they were developed to elicit debates about 'relevance' of content and pedagogies which often meant the students wanted courses that addressed matters of concern rather than formulaic recitations of content that had been taught for generations. By the 1990s that evaluation apparatus had been appropriated by universities as a way of assessing teachers. Increasingly, students see their education as 'transactional', meaning they are at the university to get a degree and to do so, they must take certain courses. They want the course syllabi to contain explicit lists of tasks which upon completion will generate certain grades. The students evaluate their courses much as they would any other consumer product. Over the last generation there has been extensive research conducted on the course evaluation process; one common finding is that women and minority faculty receive lower rankings.

Meanwhile, the rankings are carefully tabulated by the university and increasingly used in evaluating faculty. When the evaluation forms were still on paper and distributed in class, it was unacceptable at UCLA for faculty members to collect and return the completed forms to the appropriate office. There was concern that faculty members might alter the markings to improve their rankings, which apparently happened. By now it is rather clear what kind of syllabi, classroom pedagogy, and student-faculty interactions lead to high rankings which is not difficult to achieve. It is especially important for the precariat instructors and the untenured faculty to perform to those 
expectations. Sharon teaches her graduate students how to do so, although she often choses to not do so herself. Increasingly few challenge the formulaic expectations; to do that is usually seen as a tiresome waste of everyone's time, if not simply labeled pedagogic weakness by both the students and the other faculty members.

Although the relationship between excellence in research and teaching is not clear, the increasing emphasis on regulating teaching quality is causing changes in the relationship between teaching and research. NTNU's linguistic transformation of 'excellence' into 'high quality' (see Chapter 2) may moderate the strain, but not much. The faultline between research and teaching is nevertheless increasing. To some extent, this growing separation has been fueled by the rising number of PhD students, post docs, and researchers, who do research but little or no teaching. Relatively speaking, as already noticed, research has become a larger share of the activity of universities. Above all, the faultline is discursive, articulated in abstract university policies, which separates measures to improve teaching from policies aimed to support university research and improve the traffic in knowledge.

\section{Academics Circulating Through Government, and Industry}

If students are one way to circulate knowledge from universities to society, another set of encounters arise from research and exercise of academic expertise. Knut has had extensive contact with both industry and the public sector through research projects and participation in committees and giving talks at seminars at the Ministry of Climate and the Environment and the Ministry of Finance, in addition to several other directorates. Increasingly, both the Research Council of Norway and EU's Horizon programs require collaboration with non-academic organizations. Both institutions are themselves arenas where university academics meet with people from industry and the public sector. Moreover, it is noteworthy that Christensen and Holst (2017) show how the participation of university academics in public commissions in Norway has been growing considerably. This indicates increased policymaking influence of faculty.

In her visits with colleagues in Norway, the Netherlands, Denmark, and Sweden, Sharon has been impressed and intrigued by the exchanges between faculty members and policy makers at all career stages; they clearly were very well-informed about each other's work and the discussions about policymaking were sophisticated. In the US there is almost no university policymaking at the national or state level and very little policymaking about science and technology in those arenas. However, many different governmental agencies and private foundations fund university-based research. It should be remembered that there is some kind of exchange being conducted in these encounters. The funding agencies want to encourage certain kinds of research; faculty members want research funding. Those grants also are a 
significant revenue source for most universities. For example, if a UCLA faculty member receives an extramural research funding grant from a national government agency, the university receives an extra 54 percent for what are called 'facilities and administration' (F\&A) costs. If UCLA faculty members together receive grants over $\$ 1$ billion a year, then that 54 percent becomes a large number. Each university negotiates their F\&A fees separates with the government agencies; they typically range from about 50-65 percent.

Some of the funding program officers in the US are academics who have 'rotated' into those managerial positions for three years, having taken leaves of absence from their home universities (in Norway, such practice is rare). The more established, senior funding program officers at government agencies and private foundations often have left their academic positions to pursue a career in research funding. Some entered their positions directly after finishing their doctorates. In the US, discussions are held regularly between faculty members seeking such funding and those who allocate it. The conversations among the US funding program officers and academics are productive and informed; the exchanges often are informal and relaxed, held among people who might have known each other for decades. These meetings are held both at the government and foundation offices and on campus. Although most leading US universities now have extensive offices for managing extramural research funding, they are relatively uninvolved in initiating the funding process. Most of that is conducted through informal meshworks.

In general, there are active engagements at many levels between US academics and those whose offices and agencies fund university-based research. The meshwork is dense, but perhaps only about 10 percent of faculty members are acquainted with a research funding program officer. Typically, when teaching is discussed in those exchanges the interest from the funders is focused on developing and sustaining the workforce in the fields of inquiry. Since the 1990s there has been a sustained and growing interest in diversifying that workforce, especially with respect to gender, ethnicity, race, and disability. They also want to encourage 'public outreach'. Over the last 25 years most funding groups now require those topics be addressed seriously in any funding application.

Sometimes the university-based factional epistemic politics extends into the funding agencies and foundations. Some engaged in established lines of inquiry are understandably territorial about their traditional funding sources and actively work against the establishment of new funding lines for the new lines of inquiry. In that context there can be harsh relations between meshwork clusters. Part of that competition concerns the recruitment of younger scholars. Sharon knows of two such territorial issues that lasted for decades; both concerned research funding lines from government agencies which conventionally focus more on established lines of inquiry with a slow rate of change. Meanwhile, the funding program officers from the private foundations are especially eager to know about new lines of inquiry and how 
they might link to new initiatives from their agencies and foundations. Some of the foundations specialize in encouraging certain kinds of knowledge, as, for example, the Ford Foundation does in urban, gender, and ethnic studies, the Carnegie Foundation supports peace studies, the Mellon Foundation funds innovation in humanities scholarship, and the Sloan Foundation encourages public outreach in science and engineering. Clusters of faculty members and program officers can work together to build new lines of inquiry.

In Norway most university faculty members participate in such extramural meshworks - certainly to varying degrees - but these exchanges seem to be given little public notice. Probably that is because the discourse around universities' interaction with larger society's focus on deficits. The main view of research policy makers is that the interaction is insufficient and that academics are too reluctant to engage with outside actors (Åm et al., 2021). The traditional perception of the university as an ivory tower that we discussed earlier, persists and casts long shadows. Above, we referred to the vision document of the European University Association, titled 'Universities without walls'. The use of this title clearly owes to the ghost of the ivory tower, since, as we argue, universities are fairly open public spaces already. However, the deficit discourses also reflect the neoliberal acceleration imaginary, the insistence that knowledge must flow more quickly. Thus, university academics must make their flow activities more effective and efficient. From the point of view of acceleration and efficiency, university teaching is a detour. To increase the speed of knowledge flows from universities, faculty must engage more actively in innovation.

\section{Innovation - a Third Mission}

Innovation has become what some call universities' third mission, alongside teaching and research. While teaching and research are conducted at the university, most think innovations happen elsewhere, in industry and the public sector. Admittedly, universities engage in commercialization of research and the filing of patents, but universities' main contribution to innovation is their traffic in knowledge and their role as knowledge commons. This largely overlaps with the traffic emanating from teaching and research and is therefore difficult to distinguish. NTNU and UCLA face considerable challenges in making visible the active, intensive meshworking that faculty and students engage in when they contribute to the third mission. We are particularly interested in these challenges.

First, we should acknowledge that over the last generation there has emerged a large and lively global interdisciplinary research field of 'innovation studies' with many such centers at universities around the world, scholarly research journals, university press book publication series, and research funding programs. Scholars are based in departments of administration, economics, policy studies, sociology, and so on. Governments and corporations 
are actively engaged in defining their work in terms of innovation strategies. The most important source of innovation is learning from practice, from making or using technology or knowledge.

For our purposes, we find it useful to emphasize the observation by Pfotenhauer et al. (2019) as to how policies in many countries reflect an 'innovation imperative', resulting from a growing belief that innovation is key to solving pressing social, economic, and environmental problems. The innovation imperative also saturates university discourse; this is evident from the idea of innovation as their third mission. Our two universities have willingly accepted this mission and integrated it into their goals and strategies. In turn, the focus on innovation has produced a widespread concern with so-called innovation deficits, claims that the achievements of research, industry, and the public sector are insufficient. NTNU and UCLA also are seen to have innovation deficits. How do they deal with this?

The introduction of innovation as a third mission has come alongside the growing investment in university research. Government and industry require this added type of public goods to legitimize universities' growth. University teaching and research are supposed to provide flows of knowledge that increasingly stimulate innovation. As a guiding vision, research-based innovation is now on a par with research-based teaching; the unity of innovation and research complements the unity of teaching and research, potentially forming a virtuous trinity. However, the display of such virtue is difficult to prove because of the lack of metrics, given the universities' preference of numerical indicators. What do universities do when they try to stimulate innovation?

At UCLA the innovation projects typically support new ways to engage university-based research with business initiatives. UCLA has a Center for Innovation, an Innovation Fund, a Creativity and Innovation program, an Innovation Lab, an Innovation Hub, a Center for Education Innovation, a Cross-Campus Innovation Challenge, an INNOVATE@UCLA program, Faculty Innovation Fellows, and an Innovation Award. The campus-wide UCLA Technology Development Group for Innovation, Research and Entrepreneurship (TDG) is led by an associate vice chancellor and it manages UCLA's patenting procedures. In 2017 the government of the State of California allotted \$22 million equally to each of the University of California's ten campuses for Innovation and Entrepreneurship Expansion: 'UCLA will direct much of its share toward helping turn grant-funded research into marketready technology by demonstrating that ideas work as proofs of concept' ${ }^{12}$

NTNU is presently hosting 12 centers for research-based innovation (SFI) that are supported by the Research Council of Norway. The underlying intentions demonstrate the complexities with respect to what such centers are supposed to achieve:

The Centres for Research-based Innovation are to develop expertise in fields of importance for innovation and value creation. Through 
long-term research conducted in close collaboration between researchperforming companies and prominent research groups, the SFI centres are to enhance technology transfer, internationalisation and researcher training. ${ }^{13}$

The centers have been evaluated with mostly positive assessments, but are still seen to have a set of innovation deficits. 'The centres have many common innovation issues including recruitment and mobility; verification of simulation results; proof of principle research; pilot testing and the use of demonstrator projects; patenting and intellectual assets in projects; and start-ups. ${ }^{14}$ Research-based innovation is not a simple linear process, even if there are linear assumptions built into the concept.

The evaluation of the NTNU SFI centers was qualitative. However, Reuters, for example, annually publishes a ranking of the most innovative universities, based on a set of metrics. These metrics illustrates a widespread view of what counts. Reuters uses three main categories: the number of patents filed over a five-year period, the number of successful patent applications in the same period, and the frequency of academic papers originating with the institution being cited in commercial patent filings. ${ }^{15}$ Thus, innovation is reduced to patents, which clearly is a very narrow definition. It reduces innovation in university contexts mainly to commercialization.

Consequently, such innovation metrics are challenging for our universities because most innovation-related activities are not included, just as many scholarly activities are excluded from the other metric evaluation systems. Some argue that the amount of commercialized research at NTNU and UCLA is insufficient to legitimize the massive investments in their research. They need a broader understanding of how universities contribute to innovation. Arguably, they are unreflexively trapped in the metrics-based governance when it is applied to innovation, given that such activities shall amount to be their 'third mission'. With respect to innovation, universities struggle harder than is the case with teaching and research to overcome what appears as an iron cage of quantitative indicators. The problem is similar to assessing quantitatively the 'work life relevance' of graduates and the extent to which teaching succeeds as a device for traffic in knowledge. Such traffic is at the heart of universities' contribution to innovation, but how may it be accounted for and audited? How are the meshworks of students, faculty, professional associations, industrial companies, public institutions, policy makers, and so on to be measured? Can they be measured?

NTNU tries to deal with the challenge of assessing its impact on innovation in three ways. First, innovation and entrepreneurship have become topics offered to students and is the focus of one study program. Students are encouraged to become entrepreneurs in the meaning of agents of commercialization, a narrower interpretation of entrepreneur than the one we used in the previous chapter. There is also economic support available to students 
who want to try their luck at commercialization. Thus, there is a clear link between teaching and innovation, but probably the opaque connections are more important and interesting.

Second, NTNU leadership pursues a bureaucratic strategy toward the third mission in the sense that they try to demonstrate to external authorities that they actively try to fill the assumed innovation deficit by implementing visible, reportable organizational measures. This includes presenting innovation as a main goal of the university and the establishment of a strategic program for knowledge-based innovation, led by a pro-rector with a special responsibility for the program. The program includes support for faculty who want to try to commercialize their research and a technology transfer office. In addition, NTNU has employed 15 innovation managers with quite ambitious responsibilities: ${ }^{16}$

- Explore research results in depth to identify and realize ideas for potential innovations.

- Help to increase innovative activity.

- Realize research-based innovation together with partners.

- Further develop a culture of innovation.

- Increase the visibility of innovation activity.

The achievements of the innovation managers and the strategic program are unclear. However, the discursive effects are evident. Concerns for innovation and entrepreneurship have permeated the policy talk of NTNU and UCLA leadership. In this sense, the third mission has been put on the agenda. The extent to which practices have changed is an open issue. NTNU and UCLA have always been knowledge commons, with an emphasis on traffic in knowledge with industry, schools, the public sector, and so on. Probably, the traffic has increased concurrently with the growth of research activities and increasing demands from funders about engagement with 'users'.

UCLA announces on its Facts \& Figures website:

During the 2016-17 fiscal year, UCLA had a total impact of $\$ 11.06$ billion on the California economy. Over 72,700 full-time jobs throughout the state were supported by the spending activity of UCLA. In the same period, 24 startups launched using UCLA-developed technology and 251 U.S. patents were issued to UCLA. Since the year 2000, startup valuations built on UCLA's technology totaled $\$ 33$ billion. ${ }^{17}$

An NTNU study from 2017 found that in the period from 2005 to 2015, the university had entered into more than 10.000 R\&D collaborations with Norwegian and foreign private sector companies. The university had collaborated with 1200 Norwegian and 760 foreign companies; the number 
of collaborations doubled in the period. ${ }^{18}$ Given that also the collaboration with the public sector is substantial, this suggests extensive meshworking.

The bureaucratic approach acknowledges the importance of meeting places to build 'an innovation culture'. A committee appointed to make innovation more visible at NTNU also suggests the introduction of incentives to stimulate faculty's engagement with innovation and that innovation is given more weight in hirings and promotions. They also argue that NTNU needs to consider its system of work plans. Presently, the system allocates time mainly for teaching and research, which is seen to make innovation marginal (Daugstad et al., 2020).

The third strategy is indicator gaming. NTNU has succeeded in convincing the Norwegian government that the impact of universities on innovation needs to be considered more broadly than commercialization and patenting. The result is that NTNU has been given the responsibility to develop more suitable indicators to assess the efforts of Norwegian universities. Based on a comprehensive literature review (Kaloudis et al., 2019), 45 indicators have been proposed. They are classified as measures of either direct or indirect contributions. Most of the indicators measure innovation indirectly, 32 compared to 13 indicators of direct contributions. This nicely illustrates the difficulties involved. The direct indicators reflect the ranking metrics, emphasizing commercialization and patenting. The indirect indicators constitute at best circumstantial evidence, such as students' satisfaction with the emphasis on innovation in their program, the number of participants in architectural competitions, or the number of articles with co-authors from industry. Since 45 indicators are too much, the next step is a reduction. ${ }^{19}$ Still, it appears that the outcome of NTNU's effort will be that the number of innovation indicators exceeds the total number of indicators presently used to assess university teaching and research.

Sometimes UCLA points to another form of innovation: social change.

Perhaps most notably, UCLA was ranked the number one top-tier university for enrolling low- and middle-income students, as well as mobility after graduation by the New York Times in 2017. ... Specifically, UCLA was first among 65 other 'elite' schools for having a significant share of students from families making less than $\$ 20,000$ per year - a testament to UCLA's commitment to equality of opportunity. ${ }^{20}$

Increasingly, US universities try to position their own students' social and economic mobility as innovation indicators. UCLA's many innovation programs and their representation as part of the university's 'economic impact' show how the innovation discourse has come to define the universitysociety relationship. Nonetheless, the scope of how the university defines its innovation activities is limited to a narrow range of economic indicators. The strong implication is that those brought into the university to administer its 
'innovation' strategies are generating activities and then indicators that would be included in a corporation's annual report to its shareholders. They do not seem to know the university's activities very well.

NTNU's struggles with indicator gaming illustrates the difficulty with an accountability and audit approach to how universities support innovation. A typical feature of their efforts is that they reflect a linear understanding of the university-innovation relationship, which makes universities into driving actors. This seems paradoxical, given the emphasis on collaboration between university faculty and external participants. It implies a lack of understanding of how universities are knowledge commons that at least in principle are widely accessible and the way in which innovations tend to be the outcome of rhizomic flows of knowledge, needs, and experiences from many sources. Moreover, the struggles clearly indicate that when NTNU emphasizes innovation it is not as potential stream of income. It is not so much a corporatization initiative as a response to requirements from the Norwegian government.

\section{Interdisciplinarity and the Epistemic Angst of Disciplines}

In this chapter we have discussed the research-teaching relationship and university innovation strategies as part of our attention to what we have called knowledge flows into and from universities, exposing the failure of the stereotype of universities as 'ivory towers'. Here we continue our investigation of the traffic in knowledge with a discussion of interdisciplinarity. Like innovation, interdisciplinarity is a notoriously unclear concept, which might be why it is so widely used. At our universities, the word interdisciplinarity appears frequently in plans and policies without much clarification. Since our main focus is on discourse, we also use it without a clear definition and without discussing the many other concepts that sometimes are used, such as multior crossdisciplinarity. The meaning and effect of 'interdisciplinarity' will be explained when we discuss how it is employed in the setting of our two universities.

Interdisciplinarity is supposed to open the traffic in knowledge to improve the capacity of research to address social problems, including stimulating innovation. There are many prompts for interdisciplinarity from research funding sources, such as the required participation of two or more disciplines and professions, as well as the participation of non-academic actors to form transdisciplinary collaboration (OECD, 2020). There also is quite a lot of scholarly work on interdisciplinarity indicating it is invoked as an ideal in higher education and research policy discourses (Frodeman et al., 2010; Frickel, Albert, and Prainsack, 2017; Weingart and Stehr, 2000). According to Klein (1990), interdisciplinarity for some time has characterized academic fields such as area studies and urban studies, which emerged after 1945. Here, we do not aim to provide an inventory of interdisciplinary practices. Our 
attention is on the extent to which interdisciplinarity measures smooth and increase the traffic in knowledge across disciplinary boundaries, reducing the obstacles to creating spaces where flows of knowledge from different disciplines may mix, be added, or integrated. Thus, we are particularly concerned with exploring the epistemic politics of interdisciplinarity and the construction of disciplinary privilege.

Just as universities have long been caricatured as 'ivory towers' filed with professors engaged in arcane debates about less and less, the subjects of academic inquiry, conventionally called disciplines, have been represented as silos or even takobako, fishing traps that octopuses can enter, but not leave. However, like the mythical ivory towers, the disciplines are not enclosures. Even in the medieval period the old trivium (grammar, logic, and rhetoric) and quadrium (arithmetic, geometry, astronomy, and music), a curriculum designed to transmit a basic set of thinking skills and a body of information, were continuously challenged to become more practical and relevant to society. Medicine and architecture were among the early additions. Throughout the 20th century the additions of disciplines to universities have proceeded at an intense pace.

Institutionally, universities typically have added to the fields of knowledge by expanding the arenas called departments. Often the disciplinary departments established earlier have resisted the inclusion of new disciplinary departments in universities, fearing that the proverbial resource pie would be divided into more and more pieces. Often new subjects of inquiry first appear in universities as new courses and new interdisciplinary programs. If the affiliated faculty members expand their audience among students and if their field of inquiry gains more researchers who continue meeting at workshops and conferences, publishing together, establishing new curricular programs, launching new faculty positions, and building new extramural research funding streams, then those interdisciplinary groups often become disciplinedepartments. There is a lengthy list of exemplars that have emerged over the last 50 years, including environmental, ethnic, gender, communication, media, and urban studies, as well as cognitive, computer and neurosciences, as well as STS.

This development is often overlooked in university discussions, even if there is quite a lot of collaboration across disciplines, especially in the professions, such as business, education, health, law, and policy. They have a history of emerging from assemblages of theory and practical experience, as well as disciplines and even other professions. For example, engineers are supposed to know some mathematics and physics, a bit of economics, maybe also pieces of social science and law, in addition to their engineering specialties. The professions may be more accepting of interdisciplinary work because they are more engaged with pragmatic social needs and problems. The concept of transdisciplinarity is increasingly popular with policy makers because it requires bridges between academic and experiential knowledge (Nowotny, 
Scott, and Gibbons, 2001; OECD, 2020). Another extramural pressure for interdisciplinarity comes from research funding programs that see the many achievements over the last century of work conducted at the edge or beyond the disciplines. The US National Academy of Sciences has strongly endorsed such work, recommending that university leaders actively support interdisciplinarity and the scholars who pursue it. The Research Council of Norway engages in similar ways to promote inter- or transdisciplinarity.

On campus 'interdisciplinarity' is mainly invoked as a flagship concept, reiterated from the voices of extramural authorities, but as noticed, without much effort to clarify what is meant. It is commonly used simply as a label for collaboration between two or more disciplines and professions, preferably from different areas such as engineering and social science or medicine and the humanities. How such collaboration is enacted remains a mystery to other people than those actively involved. Thus, in university discourses, interdisciplinarity appears as accidental. This may explain NTNU's ongoing, strange, and very expensive experiment to facilitate interdisciplinarity. The plan is to merge the two main campuses of the university by moving all humanities and social science departments to the campus that presently hosts the engineering and natural sciences. The price tag is estimated at USD1.4 billion! The campus will be very densely populated by students and faculty. Thus, they are expected to be forced into frequent interactions, which are supposed to produce interdisciplinary collaboration from mingling. ${ }^{21}$

However, it is striking how little our universities try to learn from the experiences of faculty members who actively engage in interdisciplinary collaboration. Knut and Sharon have worked across disciplines their entire careers and presently are situated in two interdisciplinary fields, STS and Gender Studies. Sharon's doctorate is in interdisciplinary studies and Knut has participated in several such initiatives. Both of us also have experience with disciplines: Knut with sociology and Sharon with anthropology and history. We have encountered a wide variety of practices with respect to the making and exchange of knowledge, including efforts to stabilize areas of interdisciplinary inquiry in ways that emulate disciplines. This is a clear indication that many academics find being in a discipline attractive.

While interdisciplinarity in principle is accepted, in everyday practices on campus it is the disciplines that count. As mentioned earlier, the interdisciplinary programs, centers, and institutes at UCLA receive discretionary support from deans, provost, and the chancellor. Departments have fixed 'line items' in the university budget; the departments are the institutional infrastructure for disciplines and the interdisciplinary fields that are prepared to take on the form of disciplines. At NTNU, a report was published in 2021 about the future developments regarding studies in the humanities and social sciences. ${ }^{22}$ It states that the ambitions are "to educate students who with a strong disciplinary identity and skills with respect to interdisciplinary interaction contribute to create a better world' (p. 10). Thus, the future, and not 
only the past, is supposed to belong to the disciplines. Interdisciplinarity is an epiphenomenon, it is an extra skill to be added to the soup of disciplinebased knowledge. The content of this extra interdisciplinary capacity remains fairly opaque.

The defense of the disciplines and the understanding of interdisciplinarity as an epiphenomenon are outcomes of an epistemic politics where considerable efforts are made to reproduce distinctions. We have discussed such distinctions previously and noticed how they are articulated in existing academic cultures. Interdisciplinarity tends to be seen as inferior to the disciplines. We hear people referring to such work as 'lacking engagement with basic theoretical concepts', 'too applied', 'lacking in consistency', 'lacking theoretical framing', and 'lacking rigor'. The most frequently used argument is that the disciplines are the foundation of academic work, that a solid training in one discipline is needed in order to do interdisciplinary work. To some extent, such views reflect the pecking order within the disciplines, with theoretical work having the most status. Interdisciplinarity challenges such assumptions and hierarchies, triggering what we call epistemic angst, a diffuse fear of change, maybe also loss of position and resources.

We use the concept of angst because there is little reflection regarding the sources of fear. The endeavors to make interdisciplinarity inferior are a bit strange, given that so many university academics engage in diverse forms of interdisciplinary collaboration already. In particular, we noted earlier that students and early career researchers seem quite eager to take courses in disciplines other than their primary one and are intrigued by interdisciplinary collaboration. They also seem less impressed with the conventional epistemic authority of the disciplines. This may lead to changes in the epistemic politics that currently defend disciplines. There are strong drivers of such change, not the least with respect to research policy and extramural funding. Young people see this and want to participate in the growing, often applied, interdisciplinary work that funding agencies want. The disciplines see these changes too and frequently can be heard telling the students that they will never get academic positions if they do not focus on a single discipline. Sharon has learned to inquire, knowing there will be no answer: What an interesting claim! What is the basis?

We are not against disciplinary knowledge formations as such. Much important research and teaching are done within such contexts. What we question are the efforts to make distinctions, the forcefulness of the performance of boundary work, and the reproduction of internal hierarchies. This produces barriers to much-needed flows of knowledge, even within the disciplines, but not the least with respect to the much-needed traffic in knowledge between universities and society. There is a saying that 'society has problems, universities have disciplines', an articulation of the perception of disciplines as silos. This is a view shared by many policy makers, although it is a misleading overstatement of the present situation. 
The growing extramural demand and rapidly increasing student engagements with interdisciplinary research and teaching are confronted by the disciplinary recalcitrance for any change. University leaders seem incapacitated by this dilemma. NTNU's expectation that a co-location of all its faculties will provide what is needed, is quite indicative. Believing that mingling is a good strategy to initiate interdisciplinary collaboration just demonstrates a trivialization of such work as something that just happens by coincidental meetings. It overlooks the effort that is needed to discover the potential of collaboration, to find time to meet and sort out interests and contribution, to establish social bonds, and so on. Interdisciplinary collaboration requires a lot of meshworking; the construction and maintenance of rhizomic, multi-directional relationships. The importance of that meshworking receives far too little attention in the policy discourses. At most universities interdisciplinarity work cannot rely on the stable institutional infrastructure that would support those engagements. Within such constraints great time commitments are required to sustain the discretionary funding resources. Many academics are willing to transform the epistemic inventiveness of their interdisciplinary work into something more like a discipline in order to acquire the stable infrastructure of departmentalization. Confining interdisciplinarity to discretionary resource allocation severely undermines it. The disciplines win, but their angst remains; they see the extramural pressures, epistemic curiosity, and students' entrepreneurial strategic interests have not abated.

\section{Conclusion: Flows and Deficits}

At the backbone of much neoliberal interventions in universities is the assumption that universities suffer from serious deficiencies: teaching fails to properly prepare candidates for future employment, research is not effectively disseminated, too little is done to stimulate innovation, and interdisciplinarity in teaching and research remains stifled. Thus, universities face accusations of a series of deficits that they are required to fill. Even worse, seen from policy makers and other extramural stakeholders, universities seem to fail in filling the deficits effectively, at least not quick enough. Neoliberalists tend be impatient. They want acceleration.

It may be seen as surprising that universities appear fairly reserved with respect to countering the extramural deficit arguments. One explanation is that university leadership largely has appropriated the neoliberal outlook and want their university to be seen as entrepreneurial, and so on. It may also be that university leadership considers the deficit argument not as critique but as encouragement to improve; Strathern's (1997) analysis supports this view, that performance criteria and reviews are supposed to lead to searches for ways to make progress. However, we think that university leadership is unable to counter the argument because they lack sufficient and appropriate 
knowledge about how their institution works. Although there is a comprehensive scholarship on higher education, we have yet to observe that this scholarship is taken notice of and made use of in strategic decisions about the development of our universities. These decisions are usually based on metrics we do not find are grounded in proper evidence regarding the traffic in knowledge and the role of the universities as knowledge commons, as main institutions of making, exchanging, and assessing knowledge in modern society. NTNU's struggle to develop innovation indicators, discussed above, is typical of the misguided efforts to deal with deficit arguments. The importance of teaching as a channel that can inform about research goes under the radar and interdisciplinarity remains terra incognita.

It should be clear from this and other chapters in the book that we see many deficiencies with our universities. However, we argue that the neoliberal deficit thinking is making things worse. It reinforces the reliance on metrics as our universities' main source of reflection and it causes growing faultlines between research and teaching. The result is that our universities appear unreflexive. Many other organizations use research when defining and supporting best practices, as well was setting new goals. It is deeply paradoxical, and disturbing, that our universities do not make use of interdisciplinary scholarship about universities.

In this chapter we have focused on knowledge flows related to our universities' practices regarding the unity of research and teaching, plus their efforts to stimulate innovation and interdisciplinarity. We offer a two-fold conclusion. The existing knowledge flows could have been facilitated significantly by university leadership with greater appreciation of how these flows are enacted and more interest in the way that disciplines and professions provide barriers. On the other hand, universities should be credited with the intensive and comprehensive society-wide meshworking that faculty members actively engage in through their teaching and research, their various forms of engagement with the public, and their interactions with both industry and government.

\section{Notes}

1 https://ec.europa.eu/info/research-and-innovation/strategy/strategy-2020-2024_en

2 www.insidehighered.com/news/2021/05/03/biden-aims-increase-collegesuccess-62-billion-investment

3 https://eua.eu/resources/publications/969:public-funding-observatory-2020-2021part-2.html

4 www.coalition-s.org/

5 Daily Bruin, July 6, 2020: https://dailybruin.com/2020/07/06/janss-brothers-historyof-discrimination-prompts-call-for-changing-name-of-steps

6 Daily Bruin, August 7, 2020: https://dailybruin.com/2020/08/07/usac-unanimouslypasses-resolution-in-support-of-renaming-janss-steps-to-tongva-steps

7 About UCLA Conferences \& Catering, see https://conferences.ucla.edu/ and www. events.ucla.edu/plan-an-event 
8 www.eqar.eu/kb/esg/

9 The study, which is not yet published, was undertaken by Knut in collaboration with Dr Lina Ingeborgrud. It was based on interviews with 30 university professors from many different disciplines and professions.

10 www.ntnu.edu/studies/courses/HIP3000\#tab=omEmnet

11 See note 8.

$12 \mathrm{https} / /$ newsroom.ucla.edu/releases/ucla-receives-2-2-million-to-supporttechnology-transfer

13 www.forskningsradet.no/en/apply-for-funding/funding-from-the-researchcouncil/sfi/

14 www.forskningsradet.no/siteassets/publikasjoner/2019/midway-evaluation-of-17centres-for-research-based-innovation-sfi-iii.pdf

15 www.reuters.com/innovative-universities-2019

16 www.ntnu.edu/innovation-resources/knowledge-based-innovation

17 www.ucla.edu/about/facts-and-figures

18 www.ntnu.no/documents/1272503658/1276139892/Damvad_Analytics_NTNU_ Report_18.01.2017.pdf/6d3b3e8b-c562-4111-8fbe-42a84796c2df

19 NTNU's Innovation Indicators. A feasibility study. Unpublished report, Trondheim: NTNU.

20 UCLA Economic Impact Report 2018: www.ucla.edu/economic-impact/pdf/uclaeconomic-impact-report-2018.pdf

21 www.ntnu.edu/campusdevelopment

22 www.ntnu.no/fremtidenshumsam 


\section{Chapter 6}

\section{Conclusion \\ Questing a Sustainable University}

\section{Introduction}

This chapter summarizes and integrates some of our main arguments and observations, while simultaneously considering the growing focus on the need to make universities more sustainable in an environmental sense. We will explain why we see all these topics as entwined. Our universities have responded to the environmental sustainability challenges in a bureaucratic manner by issuing new regulations and establishing entities on campus that engage with environmental concerns, such as waste treatment and energy efficiency. At UCLA there is strong commitment to reducing toxic emissions and waste from the campus. At NTNU particular attention is given to reducing travel, especially air travel. We acknowledge that the growing focus on global warming and other environmental concerns has forced institutions of research and higher education to address their own climate footprints and environmental impact. This is important work that raises many significant issues to study and discuss.

However, the authoritative World Commission on Environment and Development defined sustainable development more broadly as efforts to meet the needs of the present without compromising the ability of future generations to meet their own needs. This meant that the commission integrated environmental issues with social and economic concerns (Brundtland et al., 1987). In line with this understanding, we apply a comprehensive approach to the challenges involved in developing sustainable universities. In this book we have shown that many universities have come under increasing pressure to economize in distinctive ways that threaten their relative autonomy, as well as the working conditions of faculty and students, leading to increased precarity, job insecurity, and debt. As we showed in Chapter 1, many scholars have raised concerns about this development, which threatens the sustainability of universities. Our analysis of NTNU and UCLA has revealed unsustainabilities emanating from their bureaucratizing and corporatizing governance practices, but also from unhealthy collegial relations and academic cultures. 
The Covid-19 pandemic led to a shut-down of many universities, which resulted in the replacement of physical with digital interaction in teaching and research, increased social isolation, and larger workloads. Moreover, the pandemic also disclosed serious problems regarding the economic sustainability of universities in many countries and the future employment of graduates. The recent surge of protests against racism serves as a reminder that diversity, equity, and inclusion remain a challenge to academic institutions and their sustainability in the broad sense of the concept. The pandemic has revealed again that the increasing implementation of equal opportunity, diversity, equity, and inclusion policies over the last 50 years has not eradicated the assumptions that regenerate the problems. Adding resources for the under-represented has improved the demographics, but only up to a point. Universities are a microcosm of the wider society, so universities' ongoing struggles with failures and success in recognizing and addressing the longstanding marginalizations, exclusions, and silences that have saturated their projects of teaching, research, and societal engagements are instructive. In this book we have investigated fundamental problems in how universities engage in four of its primary activities: evaluating quality, performing governance, forming academic subjectivities, and circulating knowledge. It is our argument that addressing those unsustainable practices requires engagement with marginalization, exclusion, and silencing.

When the pandemic rolls back, universities face challenges regarding the reopening of campuses. These challenges seem to be met by much uncertainty and anxiety. There are expectations about a return to normal conditions or a so-called new normal. We question the sustainability of 'normal', be it new or old. We see the experiences from the shut-down and the slow, ambivalent efforts at re-opening as providing an opportunity for critical engagement with academic institutions and practices for which this book is a contribution. This opportunity should be used to explore and discuss the needs and options for making academia sustainable in the multiple meanings outlined above. We also need to include reflections about how universities can contribute better to the traffic in knowledge that is vital to the much-needed sustainability transitions of wider society globally. We agree that universities should address social challenges through research and teaching; the issue is how this is done and the kind of meshworks that are and should be developed and employed. It should not be just governments and the rich and powerful who should define social challenges; this requires broader democratic engagement worldwide.

Thus, we pursue two main concerns in this chapter. First, we turn to unsustainabilities in academic cultures and university governance. This includes the epistemic politics of disciplines and some deficiencies in academic citizenship. Second, we raise some issues regarding the role of universities in the transnational traffic in knowledge and changes in the ecology of knowledge making that we have experienced. We also discuss some consequences 
of these changes with respect to knowledge flows related to climate and the environment. At the end, we return to some specific challenges.

In Chapter 1, we briefly commented upon some of the many existing suggestions of how universities should change to remedy their current struggles and weaknesses. The most popular one, which tends to reappear in a ghostlike fashion in many debates, is a return to what is considered as the golden age of universities. The 'golden age' is definitely a view from partial perspectives. It is what Bauman (2017) calls a retrotopia, a mode of thought that represents a nostalgic return to an abandoned past. It should be clear by now that we do not share such retrotopic thinking. We do not remember our universities as idyllic islands when we entered academic life.

The ideas of 'the entrepreneurial university' (Clark, 2004) and 'the service university' (Cummings, 1998) have paved the way for neoliberal changes and academic capitalism. They were responses to the struggles of 'golden age' institutions to adapt to the mass production of teaching and research in universities. Douglass' (2016) proposals for reforms to increase the relevance of academic work to provide for 'the new flagship university' represent an alternative approach worthy of consideration. We also see interesting efforts to discuss a multiplicity of reforms and ways of constructing universities, such as Connel (2019) and Staley (2019). Importantly, they invite reflection about institutional diversity as a positive achievement, avoiding the idea of a 'normal' university. However, we abstain from such engagements to provide blueprints of alternatives for universities alone. They are fundamentally part of society; it is problematic to discuss university reform without considering the need for broader social change. Next, we address some issues that relationship raises.

\section{Our Indicators, Ourselves}

Academic freedom is under pressure, in many countries including the US. One form of such pressure originates with austerity politics, like we see in Australia and the UK, with serious effects (Collini, 2017; Fleming, 2021). Another kind of erosion of academic freedom stems from authoritarian government politics, which intervene in the content of research and teaching. Such interventions may aim to stifle academic critique of the government and ongoing rolling back of previous social reforms. It also is striking how authoritarian governments try to remove or silence fields that do research and teaching that do not comply with nationalist, conservative ideologies, such as studies of gender, sexualities, races, and ethnicities. NTNU and UCLA do not now experience such interference, but recent developments in some states in the US and in Denmark should be a warning not to take anything for granted. ${ }^{1}$

There are many critiques of authoritarian interventions in universities and the demands for neoliberal austerity in their operation. In general, critical 
university studies tend to explain entrepreneurial, corporatizing, and bureaucratizing transformations by placing the responsibility with the government, university leadership, or both. There are good reasons to do so, but as we have shown in previous chapters, a singular focus on extramural forces means to overlook that we, faculty as well as students, participate in the changes that we criticize. This is most evident with respect to metrics and the ways in which we relate to or play indicator games and how we engage with the multitude of evaluations that are now widespread ingredients of academic life. We are complicit.

Strathern (1997) observes that the roots of the audit cultures that we today consider painful are to be found the 18th-century practices of examining students. She ironically points out that industry learned auditing and accountability from universities, and then they sent them back to universities in the latter part of the 20th century. Auditing needs criteria for performance assessments; these criteria are then used to define goals that in turn drive the idea of improvements. 'Auditing is not a foreign activity in higher education, but one that sits rather well with it' (p. 319). Auditing co-produces aims and performance measures that inspire academics for at least two reasons. The subject formation of the entrepreneurial academic makes us eager to improve, to play the indicator game, and to compete.

Fochler and de Rijcke (2017) invite STS scholars to reflect on our dual roles of criticizing the system and participating in it. To what extent has the growing number of performance metrics been gamed or sabotaged? Or have those metrics been appropriated as an instrument to monitor self-improvement? Strathern (1997) points in the latter direction when she notices the implications of measurements being turned into goals for universities, departments, faculty members, and students. When we use systems of evaluating academic performances of others, we are enticed to think about how we may improve our own performances according to the metrics. Even if we notice that the systems represent a particularly odd way of valuing academic work, we learn to feel pride in being assessed positively. We enact the entrepreneurial subjectivity that we have been formed to become, as we showed in Chapter 4. The authors of this book do not claim to be unaffected by such subject formation.

Meritocracy is at the heart of the matter. We must cope with the double bind, the simultaneous hope of being fairly recognized and the knowledge that the system is unfair and unpredictable, often unaware of what we face. The academic subject is formed to believe in success from hard work, rationality, and discipline, while accepting an inherent uncertainty about how the worth of our achievements is assessed; all that is coupled with an arrogant trust in our ability to assess the worth of the achievement of others. As objects of review, we are nervous about the outcome; as reviewing subjects we feel confident about our ability to value. Thus, academics tend to play the indicator game, rather unreflexively. We perform the epistemic politics of 
allocating worth without recognizing how we enact the authoritative voices of the masters of university governance and our disciplines.

Let us provide an example to illustrate. Some 15 years ago, the Norwegian Ministry of Research and Education introduced a ranking system to value publications, to provide metrics that could be used in the budget model that allocated money to the institutions of higher education. The most striking feature of the system was how journals and publishers were split in three levels. Level zero consisted of publication outlets that were not considered scientific, usually because of faults with their peer review practices. Most outlets with satisfactory peer review belonged to level one. Level two should consist of publication outlets that could be seen as excellent. The share of level two outlets was for unfathomable reasons set at 20 percent of the total number of items at levels one and two. The decision of which journals and publishers were to be designated at level 2 was left with the discipline and profession-based national expert committees within Universities Norway, a cooperative body for all 33 accredited universities and university colleges in Norway. Thus, it is a collegial decision. Publications at level 2 are given three times as much worth as level one publications.

This publication ranking system has been subject to a lot of controversies, but it should be noticed that unlike similar systems in many other countries it does not discriminate against the humanities and social sciences, compared to the STEM disciplines. The main focus of the resulting controversies was the idea of having a system that attributed different worth to publications, depending on the outlet as judged by the expert committees. Thus, it may seem a paradox that a noteworthy consequence of the ranking system was its impact on defining excellence in various evaluation practices. The system was intended to be used only at the institutional level, as one item out of several to assess the research performance of universities and university colleges. It was explicitly not meant to be used to evaluate individuals. However, soon this intention was perverted. In many disciplines, hiring and promotion committees began to use the distinction between level one and level two publications to value the research performance of individuals, giving much higher worth to level two books and articles. Moreover, it became more common to count rather than read publications.

This practice of doing peer review on the basis of quantitative measurements did not happen at the bequest of the government or university leadership. It resulted from faculty members' preferences and assessment practices. The socalled Declaration on Research Assessment (DORA) was launched in 2012 to counter such practices. ${ }^{2}$ Seen from the perspective of critical university studies, it appears ironic that policy makers, administrators, and university leadership have been more active than the academic rank-and-file in promoting the DORA principles for more responsible assessment practices. Metrics appear to seduce academics in yet another fashion; it simplifies assessment work and saves time. 
From our experience, the ecologies and practices of university research have changed during the last 40 years. Some of the changes are caused by the introduction of performance metrics and rankings. There is little doubt that the pressure to publish has increased. 'Publish or perish' is an old academic saying, but the new ecologies of research that are intersected by metrics have made it far more complicated. It is not enough to publish; you must publish in particular formats and channels. Format and channel strongly shape the worth of a publication, while numbers of publications and citations to them have become a proxy for quality. Academic publishing is to a worrisome degree similar to new social media practices in becoming a click economy. Thus, it has become vital for ambitious academics to learn how to be worthy of that kind of attention, to be downloaded and cited.

Good work is not sufficient to achieve this. Reviewers and publishers increasingly ask that publications demonstrate newsworthiness, while adhering to disciplinary and professional standards. This reflects the insane growth of academic publications, which makes it difficult to stand out in the crowd. There are good reasons to question the sustainability of this growth, which makes it impossible to read but a small number of the available publications in any academic field. The deluge of publications is due partly to the huge growth of the number of academics, but substantially also to the pressure from publication metrics and competition about resources and positions. Müller and de Rijcke (2017) show in their study of life scientists how publications metrics have changed the content of academic work at every stage, shaping research to yield publications that are valued by the metrics system. Sarewitz (2016) argues that the situation generally threatens the quality of research, not the least due to the widespread practice of using small samples that allow for speedier projects.

The changes in the ecology of university research appear incremental and at any given moment may not be that easy to detect, not the least for people who recently have started their research careers. However, there has been a serious transformation of the moral economy of academic research and publishing by altering the relative worth of activities and qualities of results. Arguable, the publication metrics have become a temptation to play indicator games, not only with respect to conducting research and writing papers but also in the way peer reviews are conducted, replacing thorough qualitative assessment practices with quick calculative evaluation procedures. Our teaching assignments feed the rankings, too.

We do not think it is possible to stop the use of metrics. What we need are considerations regarding what we could call a metrics morality, ensuring that metrics systems provide fair indications of quality and effort, that they are used to attribute worth fairly and sensibly, and not misused to engage in superficial assessments and hyper-publishing of micro-efforts. Thus, we need considerable improvements in the conduct of academic citizenship, since the problems are not only caused by extramural forces, even if these forces are 
very influential. Present debates about metrics are highly critical of current practices, for good reason because of the excessive, proliferating use of quantified measurements with poor validity. This malpractice is largely, but not only, caused by neoliberal regimes such as New Public Management.

We should think of alternatives, since the power games attributing academic worth in 'the good old days' were quite harmful too. It is useful to consider more carefully the diversities of metrical systems and their impact, to reflect on alternatives and inequalities. For example, it seems as if the life sciences have experienced more harmful effects than many other areas of research. The fields that practice far more open peer review, such as those employing arXiv, appear to have a more sophisticated discourse on quality. The fields with free access to scholarly publication appear to be avoiding the accumulation of epistemic authority in the usual sites of privilege. Fields with active, shared evaluation among scholars, community activists, and policy makers seem to be making work that is both original and implemented. STS researchers should know much more about these processes.

In this book, we have juxtaposed the evaluation systems at NTNU and UCLA and discovered substantial differences, both in the construction of the systems and the selection of indicators and in the ways the systems are implemented. In the case of NTNU, the main part of the metrics has been supplied by the Norwegian government, largely to oversee the production of public goods, their social relevance, and the implementation of new policies of research and higher education. The metrics were not intended for other purposes than the assessment of the university; to the extent that, for example, publication metrics are used to evaluate individuals, it happens at the initiative of faculty and only in the case of hiring and promotions. UCLA, on the other hand, has constructed its own metrics based on international and national rankings and benchmarking. The systems have been made to promote 'excellence' and they are regularly used to assess faculty members individually. Constructing metrics to incentivize social relevance has rather different effects than focusing on excellence, as we have observed repeatedly. These differences are clearly relevant when we consider sustainability issues.

\section{University Governance: Bureaucratizing at NTNU and Corporatizing at UCLA}

Throughout the book, we have emphasized the importance of carefully examining the use of stereotypes such as 'the neoliberal university' to understand the considerable variation in those practices that exists. We intended the juxtaposition of NTNU and UCLA to contribute to this. We have discussed features of the local political economies to show that context matters, and to explore how the governance of the universities can be seen as responses to those differences. We saw the strong presence of the Norwegian government 
in the funding and governing of NTNU as leading to a mainly bureaucratizing governance, based on a proliferation of policies, standards, and reporting. The bureaucratizing at NTNU thus follows a specific pattern that reflects government policies and requirements about internal quality control systems, including human resource considerations and systems to oversee that the rights of students are accommodated.

We observed UCLA to be more corporatizing, focused on managing the many complex streams of revenue on which the university depends. This is a distinct form of corporatization as we observed in Chapters 2 and 3. A significant feature is the way in which corporatizing is accompanied by specific 20th-century forms of bureaucratizing: adopting management models and software from the 1980s and 1990s when the ideal was a 'tall' hierarchical organization, just before the move to 'flat' structures required new kinds of management models and software. The old focus was on generating standardized products efficiently and managing customer relations effectively, just as UCLA's management software does when misapplied to teaching, budgeting, admissions, personnel issues, and so on. Perhaps the downsized staff from the tall corporations simply moved to the public sectors of government and universities, bringing with them their favorite models and software. From 1975 to 2015 management positions expanded 13.6 times at the University of California, while students increased two times, and tenure track faculty grew 1.6 times (Lu, 2021). Corporatizing UCLA has meant a stunningly larger administration than the bureaucratizing NTNU, which appears much leaner. We see NTNU's leanness as related to a different way of corporatizing, which mainly is a rhetorical construct to demonstrate that NTNU's leadership has picked up on the discourse of 21st-century management, such as 'serving our owners', as if state ownership were similar to any private corporation. However, the large merger of campuses in 2016 could also be considered in a corporatizing frame, given that it led to a considerable growth of the university.

'Entrepreneurial' is another stereotype that needs investigation since it too carries multiple meanings. As with performance metrics, what began as a way to describe and evaluate universities has quickly become a form of academic subjectivity. Studies of corporate entrepreneurship have been instructive in identifying different corporate ecologies that support different kinds of entrepreneurship sought by the corporation (Wolcott \& Lippitz, 2007). We also note the kinds of deviant entrepreneurship that some corporations encourage as a resource for innovation, and others, like UCLA, do not (Heracleous et al., 2018; Dahling and Gutworth, 2017; Petrou, et al., 2020). The corporate move from tall hierarchical organizations to flat ones by the beginning of the 21 st century was done to increase competitiveness and innovation in rapidly changing, global ecologies; paradoxically UCLA adopted the abandoned old model just as it was shown to be ineffective for the goals UCLA seemingly was beginning to embrace. 
We have described both of our universities as entrepreneurial, in the programmatic sense that they both make efforts to increase their revenues and improve their situation in the larger, transnational ecology of knowledge formation. With NTNU, increasing revenues mainly means finding ways to get more research funding from the Research Council of Norway and the Horizon programs of the European Union. This has meant growing administrative intervention in grant application work, including economic support for preparations. NTNU even has an employee in Brussels that is supposed to help with networking and guidance with respect to calls. We also saw in the previous chapter that NTNU has hired innovation managers, as has UCLA, to stimulate commercialization and innovation activities among students and faculty members. However, the NTNU effort is not primarily intended to increase university income, but rather to satisfy expectations from the Norwegian government.

As noted in many places in this book, UCLA depends on many streams of income that invite much more mandated entrepreneurship than simply increasing grant applications. For example, offices are established specifically for strategic interaction with potential donors, sports advertisers, event planners, and other powerful agents. UCLA also seems to require more intramural, factionalized entrepreneurship than NTNU, due to the volume of resources that are distributed through the personal discretion of administrators. We even learned from Chapter 4 how UCLA students and early career researchers face many more options that require comprehensive, individual strategic reflection and maneuvering than are needed in the Norwegian context. These might be called 'positive deviants' or 'loyal rebels with a cause', as are those building interdisciplinary projects. However, young academics at NTNU also have to learn to navigate individual career choices and funding opportunities.

\section{Academic and Administrative Cultures: Stylized Differences and Indifferences Persist}

Throughout this book we have described universities as a collection of situated activities: crossroads, borderlands, liminal space, and a venue. They are located at the faultines of political economies and epistemic projects. They are arenas for the contested performances 20th-century corporatists, mission-making bureaucracies, silo-building disciplinarians, and interdisciplinary entrepreneurs. They are agoras for utopians and pawa hara, community activists and epistemic corruption; they are hackerspaces for making new lines of inquiry. Sometimes the badly maintained privatized infrastructure generates toxic waste: massive debt and inequities. Workers at the knowledge factory make private and public goods. We might see universities as a commons built precariously on faultlines, sites in which all those activities and more are under negotiation, an unending set of co-morphing, domesticating, and 
meshworking. All that happens every day. We have argued that in its present form it is unsustainable. In the midst of that are some powerful actors. Some take the form of two cultures: the administrative and the academic. Their daily encounters are both stylized and strategic. Both ignore the unsustainable features of that world. The agnotology of their performance is breathtaking. We pause for a moment to describe the choreography.

A shared feature of the two universities is the unsustainable limitations of their governance. Both NTNU and UCLA rely on incentive systems, report regimes, and bureaucratic regulations and control, albeit not in the same manner. Governance is still constrained by the ideas of academic freedom and the embedded practice of self-management, on which the universities depend. Academic freedom is, is we have seen, saturated with bureaucratizing and corporatizing moves, sometimes also confined by extramural political interventions. Still, academic autonomy largely prevails because it is effective, even efficient, with respect to the performance of teaching and research. Moreover, we want to emphasize, it is a resource of resistance against governance. However, the imagined university of policy makers and university leadership is a powerful, make-believe form of governance, where commands are expected to flow down through the hierarchy to eventually be implemented below. To use a mathematical metaphor, the universities are overdetermined by the huge number of goals and thus allows more freedom of choice than intended by those governing. There is also a great deal of studied indifference to what different groups are doing, which leaves room for some autonomy. It should be noted that the vast, highly elaborated administrative structure at UCLA still relies on the faculty members to get the work of teaching and research done autonomously. As we have noted throughout this book, the leadership apparatus and the academic one both use the campus, but they appear to ignore each other in many of their routine activities.

The academic cultures we observe at NTNU and UCLA are shaped by their different kinds of governance and autonomy, as well as the very powerful non-academic forces at NTNU and UCLA. Those forces might be aware of these academic cultures on campus and beyond, but they do not participate in them. Dubinskas (1988) noted that in biotech start-up companies the academic researchers were seen as childish, self-absorbed nerds by the businesspeople in companies who in turn were seen by the researchers as necessary, but unintelligent devices for providing the money needed to do the research. Both groups strategically exploited the differences between them. They completely ignored each other at their peril. The same happens on university campuses where people often signal their affiliations and rank with their dress and speech, much as would happen anywhere in society. Even with all the variation among the academics and among the administrators the differences between the groups can be seen and heard easily. The performances of being in two cultures permeates university governance. 
The distinctive styles of dress and speech of the academics and the administrators display the differences and show every day and in every meeting that the two have not coalesced. Individuals from each culture clearly have moved into the other one and it is obvious when a faculty member begins the trek toward administration by the mutations in their dress and speech. Both groups are very much aware of the other group's styles and even exploit that knowledge to make decisions and interventions. Nonetheless, why has this difference between the two cultures not been muted over the last several decades of neoliberal practices?

We use 'cultures' in the plural because no cluster of academics or administrators displays a single culture, a homogeneous understanding of academic or administrative citizenship, and both include a varied set of epistemic politics. We have explored some of this diversity, with an emphasis on the practices among academics of collegial organizing with both benign and harmful aspects that are shaped by the local political economies and their local traditions. Hierarchies, boundary work, factions, and harassment need to be considered and reconsidered too because this shapes the traffic in knowledge, as we saw in the previous chapter. Although disputation is at the core of academic knowledge-making practices, those activities occur in a context, specifying what kind of disputation is to be conducted, by whom, and how. The differences between a friendly discussion in one field and another can be startling. Knowing the local rules of the game is important. Similarly, a discussion about university resources is deliberated differently by the academics and the administrators.

There are significant commonalities at NTNU and UCLA that show us we are among academics at universities. As in any community or workplace, much is conveyed discursively and in comportment. In academia there is much conformity in presentation of self, even if people are taking unusual positions in their work. Most of us recognize these clusters of norms and know what to make of them. To take a trivial example, contemporary academics tend to dress in an informal style, although there are specific variations by field of expertise. In most cases the normative style includes the requisite attitude that style is of no importance, which, of course, shows that it is. Most dress informally with an emphasis on comfort while not looking careless; the general idea is to convey a focus on the world of ideas. Overtly dressing to look particularly well dressed, fashionable, expensively attired, or sexually attractive is unwise. Administrative staff members, along with those in some of the professional schools of business, law, and medicine, tend to 'dress up' as if they worked in any big business office.

Of course, there are variations too. Those in architecture, arts, comparative literature, design, film, and theater fields tend to dress with some attention to 'edgy' fashion. In addition, there are swarms of students 18-35 years old on most campuses who have their own sartorial concerns; a certain cohort of faculty members of all ages tries to dress like the students and openly socialize 
with them, but that style is far less common now than it was in the 1960s and 1970s; even then it was often scorned by both the students and the other faculty members. At UCLA there is a commonly used distinction between 'south' and 'north' campus, with the STEM and medical fields located in the south. Similarly, at NTNU, there are two campuses, Dragvoll with the humanities and social sciences and Gløshaugen with the engineering and natural science. Students observe different dress codes and mannerisms; they joke about how easy it is to identify the 'others'. The distinction between the academics and the administrators is just as obvious.

There are differences too in the ways that the academics and the administrators talk. Nearly all academics perform formal discursive practices, including syntax and vocabulary, except for special emphasis or in casual conversation with close colleagues who are peers. Most equate the use of formal discourse with clear thinking, a huge concern of academics everywhere. Complex discursive strategies abound in academia. It is quite rare for an academic to not be orally articulate.

Some students commonly use filler words, repetitive conversation tics, and upspeak; sociolinguists argue that such speech acts are used to create and convey a sense of commonality, but they merely sound inarticulate to most of their teachers. Over the course of three decades in academia at several universities in different countries, Sharon has heard only three faculty members of any age affect such colloquial speech patterns. A common perception in academia is that such speakers are not thinking about what they are saying or rather they are displaying that their thoughts are not very well organized.

Moreover, speaking for long periods of time is widely practiced by academics and typically much attention is given to specifying caveats and nuances. In that context it would appear that 'occupying airtime' would be a device for displaying power. However, in faculty meetings, just as primate beta males make far more noise than the higher ranked alpha or silverbacks, those who 'hold the floor' are signaling an aspiration for power, not the occupation of it. Since almost all academics know very well how to speak for a long time on almost any subject, among peers the practice usually is held in check, except by those who choose the practice as a way to silence others, especially in meetings of a fixed length, or with non-academics, including administrators.

By contrast, corporate-style administrators tend to speak in short declarative sentences, invoking a lot of metaphors from the business world. In meetings they want short agendas, terse reports, and highly delimited action items. During their extensive communication with academics they maintain their distinctive, business-like speech. Sharon has noticed that academics who speak in ways that are perhaps annoying, or display extreme versions of academic discourse are deployed strategically. That is, they are invited to hold certain positions in which their predictable behavior will be used with some specific effect. One group said they regularly delegated such a colleague to 
engage with certain administrators because the officials would want to avoid the person's way of speaking as much as possible, meaning the instructions would be extremely brief. One group of US social scientists visiting policy makers in Tokyo brought with them a translator who spoke Japanese fluently with a slight distinctive accent that signaled he was a member of a group often disdained in Japan; his voice clearly distracted the Japanese which of course was the point. Sharon sees the same kinds of tactics at UCLA, displaying and exercising power discursively through appropriation of tensions between and within groups.

Thus, academic life is rife with learned, enacted commonalities and distinctions, some trivial, some consequential, often displayed discursively and through comportment, then performed strategically in ways that shape university governance. A slight shift in posture, comportment, dress, or voice, unnoticeable to outsiders, might make interactions much easier or far worse. Sharon remembers when she first heard about research on the linguistic techniques that led women and other under-represented groups to accept being interrupted and resolved to ignore them in the future. About a week later some colleagues asked why she had become so angry; it took her a while to locate the problem. We may not always be aware of these routine differences that can make a difference, but the processes are ingrained and have formed us as academic subjects. Honoring and disturbing the normal commonalities and distinctions go on every day. They also are part of commonplace, informal governance.

The different appearance and discursive styles of the administrators and academics are on display throughout any campus and have been for decades. Why is that difference maintained in such stylized ways by two groups occupying the same space for such a long time? The campus is a busy crossroads with many coming and going to share ideas. It is useful for the different kinds of people using the crossroads to be able to recognize quickly who might be available for different kinds of interactions. Between the administrators and academics it might be useful to remind each group that they all know they have a different stake in the governance of their shared homeland. There also is a certain competition about which group runs the place; neither side has ceded that argument anyplace Sharon has worked.

In the midst of their carefully maintained stylized differences, they need to have ways of anticipating each other's moves. On the academic side some of that information is shared between factions and departments, in spite of the complex differences among them described in earlier chapters. In well-functioning departments at UCLA the faculty members will share all the information everyone has gleaned about administrative decisions being considered and how they might be implemented, hopefully in time for strategic interventions. This is also Knut's experience from NTNU. In a less effective group gossip is shared only to display that the speaker has had access to someone influential. Since UCLA is highly factionalized and almost 
all decisions are made by those higher in the hierarchy with little consultation among those at lower ranks, access to many, busy gossip circuits can be extremely important channels for resourceful action and intervention.

Since senior administrators at US universities are appointed by their supervisors, faculty members are very interested in who is being considered for such positions and why; there is some expectation that with advance information the choice might be influenced by faculty members. To thwart the gossip networks sharing information, many universities have turned to professional 'executive search' companies of the last few decades to conduct their searches for suitable candidates; most of the larger firms have special sections to assist in university searches. Faculty members receive announcements that we are forbidden to share any confidential information we might learn through our extensive networks about a search and its candidates. Sharon remembers two significant cases at UCLA, one for a very high position and another at a mid-level rank, in which information leaked; in both cases that led to the formation of significant opposition and those candidates withdrew. In some cases she has been contacted by colleagues at other universities who have sought information about someone from UCLA who had become a candidate for administrative leadership at their university. Sometimes we face ethical dilemmas: do we recommend someone we would like to see leave our campus?

\section{University Governance, Gossip, Meshworks, and Change}

Of course, in any human community there is gossip. As we have noted, most academics are in local factions and also participate in distributed, translocal, meshworked professional affiliations. Most of the governance is informal and crucial information, consensus formation, and decision making circulates through gossip. In studying gossip as informal governance among physicists internationally, Sharon has found that among the US-based scientists the gossip tended to circulate most extensively within age cohorts, typically people of the same rank, while in Japan the gossip moved fastest among people of different ranks who shared the same research interests. Both kinds of circuits were needed to keep the international community informed about matters of concern, including funding for their very expensive projects, and to give everyone a voice. The formal governance structures of their university departments, international laboratories, and professional organizations did not include nearly so many people so effectively as the gossip circuits. Meanwhile, those circuits were like tracers, revealing the factions and their current relationships quite clearly.

Sometimes the gossip points to wrongdoing in the distributed community. Sharon remembers a case of physicists widely gossiping about a prominent colleague who repeatedly violated community codes of conduct. They 
tried to contain the damage by 'spreading the word' but the damage itself was not stopped. Similarly, news about sexual and racial harassment normally circulates in academia, like any other community, through what is often called 'the whisper culture'. The internet and social media have amplified the circulation. The point is that universities as institutions and the local academic cultures have not controlled abusers, bullies, and harassers adequately, so word about them circulates among the possible objects of that violence as a mutual aid practice.

However, there are some signs of a shift in academic communities to control damage beyond the conventional use of gossip to share crucial information and circulate warnings. For example, the last few years a few fields, such as astronomy, have begun to see the ongoing practice of sexual harassment as a failure of leadership and are addressing it, as such. Similar changes are beginning to appear concerning racial harassment. The university offices officially designated to deal with harassment, diversity, equity, inclusion, and so on, along with the whisper culture, normally address these problems as a matter of bad actors and specific cases, rather than as pawa hara, power harassment, as a problem with the state of the commons. Pawa hara is a problem because the unbridled exercise of toxic power has been both accepted and silenced, designated a personal problem, not as an unsustainable communitywide problem, an attack on all to be addressed by all. It is possible that there is a growing sense that there is an academic commons that needs far better governance to achieve sustainability.

As a form of governance, negative gossip travels very fast in academic networks, including internationally: if we gossip about someone on campus, a social event, or at a conference, we know that person probably will hear about the gossip within a week at most, no matter where we are in the world, so gossip is a way of conveying a harsh message indirectly while involving as many people as possible to circulate the news. Some chose to use those channels frequently in order to maintain and expand their circuits, rather like having followers in social media, but, of course, the risk that the message will be repurposed is very large. Consequently, those with the most and least power tend to use gossip very little, but hear the most. Factions mark the boundaries of informal conversation and gossip circuits. Partly because they are not members of any faction so seen as not being able to make use of the gossip, the marginalized are hearing stories from many groups and often are the best informed. Every society needs marginalized people to keep some lines of communication open, if only for times of crisis. Much is exchanged via meshworks, webs of relations, some of which stay within the factions, while others link some of the widely dispersed factions, on campus and beyond. Much academic and university governance is conducted through gossip.

Having noted these informal, conventional markers of difference and informal, widespread governance procedures, how might they be invoked for 
substantial change? Sometimes there are signs of change in those ordinary activities and the assumptions embedded in them, as with the growing attention to pawa hara described earlier in this book, a recognition that unbridled power, abuse, harassment, and bullying that has been tolerated must stop.

Other practices persistently challenge the commonplace assumptions of academia, as does interdisciplinary collaboration, refusing the belief that thinking and knowledge making are properly done within disciplines by individuals who take private ownership of their ideas. As we saw in the previous chapter, distinctions are often co-morphed with epistemic authority. In interdisciplinary work, this may become critical because of the need to exercise trust in knowledge made by partners from other disciplines and professions, knowledge that we lack the expertise to judge. Epistemic authority is often combined with arrogance. Academics are widely known to be arrogant, some fields more than others. However, arrogance is a barrier to the flowing of knowledge, so we may need considerable time in collaboration to overcome this. Since epistemic authority is so often exercised with arrogance, we sometimes see it replaced by epistemic charisma. Such charisma may have several sources; obvious ones are position, age, and gender. It may also stem from personality and performance, for example in the ability to explain knowledge across disciplinary boundaries or strategies for bridging knowledges or finding compromises.

Thus, when we foreground similarities and distinctions within and between academic cultures, as well as the university administration cultures, it is because these practices shape the ability of universities to serve social needs and contribute to solving important challenges. Furthermore, the cultural differences between the corporatizing administrators at UCLA, the bureaucratizing officials at NTNU, and the academic cultures at each campus are functioning alongside each other, more or less effectively at different times. They can serve as faultlines everyone knows are there, but momentarily chooses to ignore or suddenly appropriate strategically. As we have argued, some features of each of these academic cultures, administration cultures, and the collegial organizing within them are not sustainable. They are harmful and painful, making universities into places where the working environment is unacceptable and unproductive. In turn, this impedes the job universities need to do to be a providing partner in the traffic in knowledge and people that is required for the sustainability transitions our societies must make. How can universities become better partners? Given that the faultline on campus between the academic cultures and the administrative cultures has been growing dramatically at neoliberal universities, have their binary differences become unsustainable? Could the dyadic groups be separated effectively? In the midst of such normative performances of agnotology, what are the strategies for sustainability? 


\section{Knowledge Traffic for Sustainability Transitions}

When John Desmond Bernal in 1939 published his book The Social Function of Science, he provided strong arguments that science needed to be directed at solving pressing social problems, such as housing and poverty. This view clearly clashed with the widespread ideals of basic, curiosity-driven science that was vigorously defended by Michael Polanyi (1962). The controversy is still with us, the irony being that the views of the Marxist Bernal largely have been appropriated by the research policy establishment, not those of the conservative Polanyi (Pielke, 2014). As a general point of departure, we side with Bernal. The sustainability of universities definitely hinges on their ability to provide for sustainability transitions in and of society.

As we discussed briefly in the previous chapter this is not a well-defined challenge. With respect to sustainability, universities face many requirements and inquiries that need critical assessment. Current debates about sustainability transitions often ask for research to provide fixes, be they technological or social. Many academics seem fascinated by the idea of supplying fixes, in part because this appears as key to access more resources. Such temptations are the basis of the all too frequent overselling of science, making promises that we cannot keep. The experiences from previous failures, such as the 'war on cancer', provide insufficient warnings; money kept coming, as has the promissory discourse (Fortun and Fortun, 2005).

The thinking behind fixes is linear and places university research at the supplying end of the chain. At the opposite end of that spectrum technological determinism reigns. The belief in fixes require that people act according to the designers' intentions. Thus, universities face a sugar-coated trap. University research risks being held responsible for fixing problems that universities cannot fix. The danger lies in thinking that the relevant traffic in knowledge can be assumed to be one way. This may seduce arrogant academics who want to make a difference. We may recognize the slogan from the 1933 Worlds' fair in Chicago in 1933 'Science finds, Industry applies, Man conforms'.

We know this is not how it works. The predominant traffic in knowledge is and must be multi-modal and multi-directional because it is based on meshworks. Universities need to become more modest, but not obedient, more inclusive, and sensitive, more dialogic. They must change their identity from knowledge supplier to a public space intersected by a multitude of streams of many origins that require sorting out and integration. It means to interact humbly. Humility is not a common quality among academics. The more conventional arrogance is reinforced by the kind of epistemic politics where the ostensible aim is to maintain disciplinary standards and privilege, not to pursue social relevance.

In Chapter 5, we looked closely at some features of how universities engage in the traffic of knowledge. We refuted a set of claims that universities have been deficient in providing knowledge for society. First, we 
highlighted education as a main, but often overlooked channel of communicating research to wider society. Graduates of higher education are carriers of knowledge; many also have learned how to identify, assess, and even generate knowledge. Their education informs them as employees and citizens as well. Second, we observed how innovation has become a third mission where university research is expected to contribute directly to commercialization and new products and processes in industry and the public sector. This reflects the innovation imperative that dominates research policymaking (Pfotenhauer et al., 2019), which make universities look deficient. The ensuing deficit claims may make universities too focused on linear thinking. Third, we discussed how interdisciplinarity is celebrated in policy discourses, but challenged in paradoxical ways through intramural practices that prioritizes the reproduction of the disciplinary structure of university research and teaching. We showed that universities are not so deficient in making knowledge available in society as neoliberals claim. However, the traffic in knowledge is hampered by universities' lack of reflection about how they engage with knowledge making and circulation, such as insisting that disciplines are at the backbone of interdisciplinary inquiry, and in uncritical efforts to please policy makers and other stakeholders.

Our two universities clearly engage in the traffic of knowledge relevant to sustainability transitions in society and they receive considerable funding to do so. They do research in areas such as sustainable energy, climate change mitigation, adaptation to climate change, urban sustainability, and so on. The list is long and has grown a lot in recent years. The same has happened with many other fields. The increased funding has been accompanied by great expectations and deficit imaginaries. Is there overinvestment in research? Is the growth in funding, including fields focusing on sustainable issues, not sustainable? Compared to austerity politics, growth seems great. However, we need to consider if universities have entered a Faustian pact. Are our universities selling unfounded hope? What happens when they are called to deliver and fail?

We think that such questions need to be discussed. We do believe that our universities provide knowledge that is potentially important to sustainability transitions. However, we suspect that too much is expected of science, that academia has oversold. Bernal argued that science should be useful, but he did not address how and to what extent it might solve social problems. The linear model of innovation, the belief that research drives the development of new technologies and practice, has been widely criticized as wrong, but it is still used as a premise of university promises. This belief is at the core of unsustainable arguments about how university efforts will be drivers of sustainability transitions. This belief needs to be undone for universities to deliver on at least some parts of their promises. Furthermore, the bureaucratizing and corporatizing practices are undermining the more effective sustainability work that is generated at the knowledge commons. 


\section{Engaging with STS and University Studies: Some Final Reflections on Studying Unsustainable Universities}

We stated in Chapter 1 that this book intervenes in both the studies of universities and STS scholarship. Our analysis of universities, their knowledge making, and the traffic in knowledge that happens there complements and furthers the understanding in STS scholarship about these activities. While there are exceptions, there has been insufficient reflection in STS about what it means to conduct research in the context of universities, where teaching is a significant part of the researchers' jobs. Students tend to be absent in science studies accounts. In many ways, so has the quality of work life of researchers. Abuse, bullying, harassment, and power games have had little consideration within STS. There has been much attention to negotiations among epistemic claims and recruitment to support such claims, but there has been little attention to power: how it is built, maintained, challenged, transferred, or repudiated, and how this shapes the making of knowledge. The arenas can range from the quite local to the global; the time frame can be a series of momentary micro-aggressions to the launching and maintenance of imperial power, all of which can shape knowledge making profoundly as decades of scholarship in other fields has shown. Similarly, there has been little STS work on trust, altruism, sharing, and cooperation in knowledge-making processes, locally to globally.

For decades many STS scholars have done research on both infrastructure, including resilience and transformation, and sustainability, not the least climate change and energy. The infrastructures and sustainability of universities have received little attention. When we ask what a sustainable university is, it would be easy to point out that some have lasted nearly a thousand years. However, we have argued that contemporary neoliberal universities are unsustainable in many ways and have been so for decades: financially, managerially, pedagogically, and intellectually, whether in the bureaucratizing or corporatizing mode.

The consequences of the implementation of neoliberal university management are different in Norway and the US. For decades about half of all US higher education students have been taught by precarious instructors, the 'migrant workers of academe', people with doctorates who teach course by course, year by year, at low wages without health, unemployment, or retirement benefits. Very few are unionized. Student fees and debt have risen radically; adjusting for inflation, the current cost is more than ten times the fees Sharon paid as an undergraduate. Students are encouraged to take on massive debt as an 'entrepreneurial investment' in their futures (Petersen, 2021). In Norway the government requires universities to reduce their use of temporary employment and students pay no tuition. Still, when they graduate, Norwegian students also are left with considerable debt. Our book has added to that list of concerns, including the use of metrics and the relative growth 
of administrative positions, as we have in previous chapters, to question the sustainability of present developments with universities.

As previously noticed, the Covid-19 pandemic has made visible financial unsustainabilities. They are particularly prominent where universities depend on student fees and fees for university services. NTNU does not; being funded by the government makes a difference. UCLA tries to compensate by relying on other streams of revenue, but they have been disrupted for 15 months. It is unclear if the old revenue streams can be regained. As long as demands for the eradication of racism and other inequities can be met with the addition of a few new resources, the routine allocation of resources can be maintained. Where is the tipping point? Like global warming and radically widening income inequities, university audit cultures look increasingly precarious in contexts where governments are 'absent'.

Looking backward, we can see that the older, prominent, rich US universities accumulated huge monetary endowments that they hoarded for hard times; in the old slogan of the wealthy, never spend your capital; live on the interest. In good times the rich universities used their endowment income to live well. They used those hoarded funds during the 1930s global depression to pay salaries, fund students, and construct new buildings cheaply, sustaining the local labor supply. However, even the old, rich US universities stopped hoarding a generation ago; they have been using their endowments for operating expenses for decades (Goetzmann and Oster, 2014). With blind peer review on funding applications the older US universities' originally high share of extramural research funding declined. As rankings of graduate school programs became so visible, their share in educating the future professoriate and other meritocratic elites declined, too. They had prepared for the extreme cycles in the manufacturing-based political economy, but did not prepare for a rupture in the faultlines of that economy, nor the emergence of a global knowledge-based economy.

In Chapter 1 we referred to Hugh Gusterson's (2017) call for 'homework', for rigorous studies of universities. His provocation was directed at anthropologists but could just as easily be directed at STS. It is tempting to ask provokingly if universities have been seen as too mundane or too political for STS scholars to study, leading to insufficient consideration of universities as knowledge commons, as places where knowledge is constructed and processed to be shared, stored, synthesized, and transformed. This makes them critical places in society, where the search for new knowledge is intersected by social interests and provocative pedagogical concerns, potentially making them into 'devices of relevance', such as responding to the call for sustainability work, as articulated by many, including students. As we have shown, research-teaching assemblages are vital to the social functions of scholarship, including science and technology, not only as mechanisms for distributing knowledge but also as practices of validation, valuation, and social worth. In the everyday settings of universities, these practices appear 
mundane, bordering on the trivial, but we need to explore and explain them. This is no less important when innovation has become a third mission of universities.

We believe that STS scholarship provides crucial resources for analyzing and assessing the sustainability of universities, including how they contribute to sustainability transitions. We think the transition challenge is important, not the least because we see the need to consider the co-morphing of the institutional sustainability of universities and their contribution to transitions. The book is written in response to this challenge. Here, we have outlined a series of ruptures in the academic practices of evaluating quality, university governance, academic subjectivity, and intellectual mobilities; we have shown the strategic academic work of the concepts of domestication, faultlines, comorphing, and meshworking. We have argued that the astounding avalanche of corporatizing administrators arriving at the University of California since 1975 (Lu, 2021) and the provocative proliferation of bureaucratic directives at NTNU demonstrate a pathology of unsustainable growth. Neoliberal university governance is not sustainable; what is to be done?

We have identified a series of potential concerns. One of them is governance. Universities used to be quite flat organizations, with stronger governance by scholars than professional managers. So-called knowledge intensive companies, like Google and Facebook, have become flat and lean, with lateral ties and management models that have retained aspects of what universities used to be. Meanwhile, universities have copied the hierarchical models of mass-production companies of the 1970s.

Neoliberal universities defiantly marginalize or exclude the kinds of knowledge practices needed to address the major challenges faced by society globally. There is a very large and growing set of studies conducted over the last 50 years about the co-production of imperialist, ethnicist, racist, sexist, classist, ableist, nationalist, and epistemic authority, privilege, and entitlement. All justified inequities the same way. A learned indifference, a studied ignorance is carefully cultivated about the violent inequities used in the production of authority, privilege, and entitlement. Some have studied that deliberate production of ignorance to achieve and maintain power (Proctor and Schiebinger, 2008). Others investigate the compulsory silence about how such power is made. The violence of the demand for silence about what we all know has been studied by many, as well. Enforcing the silence about the making of power is the work of bullies, a cadre of symbolic violence enforcers. Even in universities, the historically designated site for disputation, there are some subjects that cannot be discussed. There is an expanding body of scholarly work decrying a major symptom of neoliberal universities: the knowledges that are defiantly excluded from them, along with chronicling the hard work of continuing to articulate that work (Chatterjee and Maira, 2014).

There is far less work on the mundane, everyday routines of neoliberal universities to launch, expand, and maintain that power, a task we have 
undertaken in this book. Our larger goal has been to show how our universities are unsustainable and identify solutions. If we seek sustainable universities, what should we do? Increasingly, environmental and ecology studies show that uncontrolled pollution leads to collapse within the next generation, with a 'tipping point' in about ten years; the primary variable that must be controlled immediately is pollution and to do that we must limit our addiction to growth (Herrington, 2021; Bateson, 1972/2000). We have shown that the analog of pollution in universities is a tightly woven web of metrics in the form of rankings and goal-speak, inequities (sexism, racism, ableism, classism, and pawa hara bullying), and factional distribution of resources. The analog of growth is the expansion of corporatizing administrators, missionmaking bureaucrats in their addictive quest for rankings and goal-speak.

To achieve sustainability we must end our addiction to our 'fixes' (rankings and goal-speak) and linear thinking by corporatizing administrators, missionmaking bureaucrats, and their enablers. To make universities sustainable in an unsustainable society seems like the work of Sisyphus; never-ending. We would like repairs, for example regarding metrics-based governance, discretionary distribution of resources, excellence considerations, academic citizenship, and disciplinary practices. We must focus on strengthening the university commons, a crossroads where faculty members, students, community members, governments, and industry meet, negotiate, and exchange our emergent, interdisciplinary knowledges. We want to foreground the meshworking, co-morphing, domesticating that lace together the knowledgemaking practices at the intersecting faultlines called universities.

\section{Notes}

1 www.thelocal.dk/20210609/analysis-why-are-denmarks-politicians-criticising-thecountrys-university-researchers/ (accessed June 24, 2021).

2 https://sfdora.org/ 


\section{References and Further Reading}

Aberbach, JD, and Christensen, T (2018). 'Academic autonomy and freedom under pressure: Severely limited, or alive and kicking?' Public Organization Review, 18(4): 487-506.

Åm, H (2019). 'Limits of decentered governance in science-society policies'. Journal of Responsible Innovation, 6(2): 163-178.

Åm, H, Solbu, G, ad Sørensen, KH (2021). 'The imagined scientist of science governance'. Social Studies of Science, 51(2): 277-297.

American Association of University Professors (2017). 'Trends in The Academic Labor Force, 1975-2015'.

Anderson, BR (1983, reprinted in 1991, 2016). Imagined Communities: Reflections On the Origin and Spread of Nationalism. London: Verso.

Arendt, H (1964). Eichmann in Jerusalem: A Report on the Banality of Evil. New York: Viking Press.

Ashmore, M (1989). The Reflexive Thesis: Writing Sociology of Scientific Knowledge. Chicago: University of Chicago Press.

Ashmore, M, Wooffitt, R, and Harding, S (1994). 'Humans and others: The concept of "agency" and its attribution. American Behavioral Scientist, 37(6): 733-740.

Baldwin, RG, and Chronister, JL (2005). 'What happened to the tenure track?' In: The Questions of Tenure, Chait RP (ed). Cambridge, MA: Harvard University Press, pp. $125-159$.

Bateson, G (1958). Naven: A Survey of the Problems Suggested by a Composite Picture of the Culture of a New Guinea Tribe Drawn from Three Points of View. Stanford: Stanford University Press.

Bateson, G (1972+2000). Steps to an Ecology of Mind: Collected Essays in Anthropology, Psychiatry, Evolution, and Epistemology. Chicago: University of Chicago Press (2000) (Chandler, 1972).

Bateson, G (1979). Mind and Nature: A Necessary Unity. New York: Bantam Books.

Bauman, Z (2017). Retrotopia. Cambridge: Polity.

Bear, L and Mathur, N (2015). 'Introduction: Remaking the public good: A new anthropology of bureaucracy'. The Cambridge Journal of Anthropology, 33(1): 18-34.

Beck, U (1997). 'Subpolitics: Ecology and the disintegration of institutional power'. Organization \& Environment, 10(1): 52-65.

Becker, P and Clark, W (eds) (2001). Little Tools of Knowledge: Historical Essays on Academic and Bureaucratic Practices. Ann Arbor: University of Michigan Press. 
Berman, EP (2011). Creating the Market University: How Academic Science Became an Economic Engine. Princeton: Princeton University Press.

Berman, EP and Paradeise, C (2016). 'Introduction: The university under pressure'. Research in the Sociology of Organizations, 46: 1-22.

Bernal, JD (1939) The Social Function of Science. London: Routledge and Kegan Paul.

Bleiklie, I (2018). 'New public management or neoliberalism, higher education'. In: Shin, JC and Teixeira, P (eds) Encyclopedia of International Higher Education Systems and Institutions. Dordrecht: Springer, section N, pp. 1-6.

Bos, C, Walhout, B, Peine, A, and van Lente, H (2014). 'Steering with big words: articulating ideographs in research programs'. Journal of Responsible Innovation, 1(2): 151-170.

Bourdieu, P (1988) Homo academicus. Stanford: Stanford University Press.

Brundtland, GH (1987). Our Common Future. Oxford: Oxford University Press.

Brunsson, N and Sahlin-Andersson, K (2000). 'Constructing organizations: The example of public sector reform'. Organization Studies, 21(4): 721-746.

Burgelman, RA (1983). 'Corporate entrepreneurship and strategic management: Insights from a process study'. Management Science, 29 (12): 1349-1364.

Burrows, R (2012). 'Living with the h-index? Metric assemblages in the contemporary academy'. The Sociological Review, 60(2): 355-372.

Calhoun, C (2006). 'The university and the public good'. Thesis Eleven, 84: 7-43.

Chatterjee, P and Maira, S (eds). (2014). The Imperial University: Academic Repression and Scholarly Dissent. Minneapolis: University of Minnesota Press.

Christensen, T (2011). 'University governance reforms: potential problems of more autonomy?' Higher education, 62(4): 503-517.

Christensen, J and Holst, C (2017). 'Advisory commissions, academic expertise and democratic legitimacy: the case of Norway'. Science and Public Policy, 44(6), 821-833.

Clark, BR (2004). Sustaining Change in Universities. Maidenhead: Open University Press.

Clark, W (2008). Academic Charisma and the Origins of the Research University. Chicago: University of Chicago Press.

Collini, S (2017). Speaking of Universities. London: Verso.

Collins, HM, and Kusch, M (1998). The Shape of Actions: What Humans and Machines Can Do. Cambridge, MA: MIT Press.

Collins, PH (1997). 'Truth and method: Feminist standpoint theory revisited: Where's the power'. Signs, 22(1): 375-379.

Connell, R (2019). The Good University. What Universities Actually Do and Why it's Time for Radical Change. London: Zed Books.

Coser, LA (1974). Greedy Institutions. Patterns of Undivided Commitment. New York: The Free Press.

Covin, JG, and Miles, MP (1999). 'Corporate entrepreneurship and the pursuit of competitive advantage'. Entrepreneurship theory and practice, 23 (3): 47-63.

Crow, MM, and Dabar, WB (2015). Designing the New American University. Baltimore: Johns Hopkins University Press.

Cummings, WK (1998). 'The service university in comparative perspective'. Higher Education, 35(1): 1-8.

Czarniawska, B (2008). A Theory of Organizing. Cheltenham: Edward Elgar. 
Daenekindt, S and Huisman, J (2020). 'Mapping av scattered field of research on higher education: A correlated topic model of 17,000 articles, 1991-2018'. Higher Education, 80: 571-587.

Dahling, JJ and Gutworth, MB (2017). 'Loyal rebels? A test of the normative conflict model of constructive deviance'. Journal of Organizational Behavior, 38:1167-82.

Davies, SR (2020). 'University communications as auto-communication: the NTNU "Challenge Everything" campaign'. Journal of Communication Management, 24(3): 227-243.

Davies, SW, Putnam, HM, Ainsworth, T, Baum, JK, Bove, CB, Crosby, SC, ... and Bates, AE (2021). 'Promoting inclusive metrics of success and impact to dismantle a discriminatory reward system in science'. PLoS biology, 19(6), e3001282.

Deleuze, G and Guattari, F (1983). Anti-Oedipus. Capitalism and Schizophrenia. Minneapolis, MN: University of Minnesota Press.

DiMaggio, PJ and Powell, WW (1983). 'The iron cage revisited: Institutional isomorphism and collective rationality in organizational fields'. American Sociological Review, 48(2): 147-160.

Doing, P (2004). " "Lab Hands" and the "Scarlet O": Epistemic politics and (scientific) labor'. Social Studies of Science, 34(3): 299-323.

Donoghue, F (2008). The Last Professors: The Corporate University and the Fate of the Humanities. New York: Fordham University Press.

Douglass, JA (2000). The California Idea and American Higher Education: 1850 to the 1960 Master Plan. Stanford: Stanford University Press.

Douglass, JA (ed) (2016). The New Flagship University: Changing the Paradigm from Global Ranking to National Relevancy. New York: Palgrave Macmillan.

Drucker, P (1954). The Practice of Management. New York: Harper.

Dubinskas, FA (1988). 'Janus organizations: Scientists and managers in genetic engineering firms'. In: Dubinskas, FA (ed). Making Time: Ethnographies of High-technology Organizations. Philadelphia: Temple University Press, pp. 170-232.

Dubinskas, FA and Traweek, S (1984). 'Closer to the ground: A reinterpretation of Walbiri Iconography'. Man 19: 15-30.

Daugstad, K, Boks, C, Falch, E, Nisja, R, Nuijten, A, and Pukstad, B (2020). Dokumentasjon og synliggjøring av innovasjon og nyskaping ved NTNU. Fokus på samfunnseffekt og samarbeid med arbeidsliv. Available from www.ntnu.no/ documents/1272503658/1276139892/2020_nov_Arbeidsrapport_Dokumentasjon_ synliggj $\% \mathrm{C} 3 \% \mathrm{~B} 8$ ring+av+innovasjon+og+nyskaping+ved+NTNU_ferdig-fil.pdf/ f109d5cd-4c3d-2fca-55b2-e15cd4e35825?t=1618922460787

Elken, M and Wollscheid, S (2016). 'The relationship between research and education: typologies and indicators. A literature review'. NIFU Report 2016: 8. Oslo: Nordic Institute for Studies in Innovation, Research and Education.

Espeland, WN and Sauder, M (2007). 'Rankings and reactivity: How public measures recreate social worlds'. American Journal of Sociology, 113(1): 1-40.

Espeland, WN and Sauder, M (2016). Engines of Anxiety: Academic Rankings, Reputation, and Accountability. New York: Russell Sage Foundation.

Etzkowitz, H and Leydesdorff, L (2000). 'The dynamics of innovation: from National Systems and "Mode 2" to a Triple Helix of university-industry-government relations'. Research Policy, 29(2): 109-123.

European Commission, Horizon 2020 https://ec.europa.eu/programmes/horizon2020/ en/what-horizon-2020 (accessed September 22, 2021). 
Evans, M (2004). Killing Thinking: Death of the University. London: Continuum.

Fanon, F (1963), Wretched of the Earth. New York: Grove Press.

Felt, U and Fochler M (2012). 'Re-ordering epistemic living spaces: On the tacit governance effects of the public communication of science'. In: Rödder, S, Franzen, M, and Weingart, P (eds). The Sciences' Media Connection - Public Communication and Its Repercussions. Dordrecht: Springer, pp. 133-154.

Felt, U, Igelsböck, J, Schikowitz, A, and Völker, T (2013). ,Growing into what? The (un-) disciplined socialisation of early stage researchers in transdisciplinary research'. Higher Education, 65(4), 511-524.

Fleming, P (2020). 'Dark academia: Despair in the neoliberal business school'. Journal of Management Studies, 57(6): 1305-1311.

Fleming, P (2021). Dark Academia: How Universities Die. London: Pluto Press.

Fochler, M and De Rijcke, S (2017). 'Implicated in the indicator game? An experimental debate'. Engaging Science, Technology, and Society, 3, 21-40.

Foucault, M (1977). Discipline and Punish: The Birth of the Prison. London: Allen Lane.

Fortun, K and Fortun, M (2005). 'Scientific imaginaries and ethical plateaus in contemporary US toxicology'. American Anthropologist, 107(1), 43-54.

Frank, DJ and Gabler, J (2006). Reconstructing the University: Worldwide Shifts in Academia in the 20th Century. Stanford: Stanford University Press.

Frank, DJ and JW Meyer (2007). 'University expansion and the knowledge society'. Theory and Society, 36: 287-311, 2007.

Frickel, S, Albert, M, and Prainsack, B (2017). Investigating Interdisciplinary Collaboration: Theory and Practice Across Disciplines. New Brunswick, NJ: Rutgers University Press.

Frodeman, R, Klein, JT, Mitcham, C, and Holbrook, JB (eds) (2010). The Oxford Handbook of Interdisciplinarity. Oxford: Oxford University Press.

Fromm, E (1941). Escape from Freedom. New York: Farrar and Rinehart.

Furstenberg, F (2020). 'When University Leaders Fail', Chronicle of Higher Education, 19 May. Available from https://www.chronicle.com/article/when-university-leaders-fail

Gill, RC (2009). 'Breaking the silence: The hidden injuries of neo-liberal academia'. In: Flood, R and Gill, RC (eds). Secrecy and Silence in the Research Process: Feminist Reflections. London: Routledge, pp. 228-244.

Gill, RC (2014). 'Academics, cultural workers and critical labour studies'. Journal of Cultural Economy, 7(1): 12-30.

Godin, B and Gingras, Y (2000). 'The place of universities in the system of knowledge production'. Research Policy, 29(2): 273-278.

Goetzmann, WN and Oster, S (2014). 'Competition among University Endowments'. in How the Financial Crisis and Great Recession Affected Higher Education. Chicago: University of Chicago Press (pp. 99-126).

Gornitzka, Å, Kyvik, S, and Larsen, IM (1998). 'The bureaucratisation of universities'. Minerva, 36: 21-47.

Gu, DY (2016). Chinese Dreams? American Dreams?: The Lives of Chinese Women Scientists and Engineers in the United States. New York: Springer.

Gumport, PJ (ed). (2007). Sociology of higher education: contributions and their contexts. Baltimore: Johns Hopkins University Press.

Gumport, PJ (2019). Academic Fault Lines: The Rise of Industry Logic in Public Higher Education. Baltimore: Johns Hopkins University Press. 
Gunnes, H (2018). 'Tidsbruksundersøkelse for universiteter og høgskoler. En kartlegging av tidsbruk blant vitenskapelig og faglig tilsatte i 2016'. Working paper 2018:2. Oslo: Nordic Institute for Studies in Innovation, Research and Education.

Gusterson, H (2017). 'Homework: Toward a critical ethnography of the university: AES presidential address, 2017'. American Ethnologist, 44(3): 435-445.

Hackett, EJ (1990). 'Science as a vocation in the 1990s: The changing organizational culture of academic science'. Journal of Higher Education, 61(3): 241-279.

Hackett, EJ (2014). 'Academic capitalism'. Science, Technology, \& Human Values, 39(5): 635-638.

Halffman, W and Radder, H (2015). 'The academic manifesto: From an occupied to a public university'. Minerva, 53(2): 165-187.

Haraway, D (1988). 'Situated knowledges: The science question in feminism and the privilege of partial perspective'. Feminist Studies, 14(3): 575-599.

Hardin, G (1968). 'The tragedy of the commons'. Science 280: 682-683.

Harding, S (2009). 'Standpoint theories: Productively controversial'. Hypatia 24(4): 192-200.

Hartsock, N (1987). 'The feminist standpoint: Developing the ground for a specifically feminist historical materialism'. In: Harding, S (ed). Feminism and Methodology. Bloomington, IN: Indiana University Press, pp. 157-180.

Henry, FW (1983). Toughing It Out at Harvard Business School: The Making of a Woman MBA. New York: Putnam.

Heracleous, L and Robson, D (2021). " "Positive deviants": Why rebellious workers spark great ideas'. Available from www.bbc.com/worklife/article/20210528-positive-deviantswhy-rebellious-workers-spark-gr-ideas

Heracleous, L, Terrier, D, and Gonzalez, S (2018). 'The reinvention of NASA'. Harvard Business Review, 18 April. Available at: https://hbr.org/2018/04/the-reinvention-ofnasa (accessed 5 February 2020).

Herrington, G (2021). 'Update to limits to growth: Comparing the World3 model with empirical data'. Journal of Industrial Ecology, 25(3), 614-626.

Hey, T, Tansley, S, and Tolle, K (eds) (2009). The Fourth Paradigm: Data-Intensive Scientific Discovery. Microsoft Research, available from http://research.microsoft. com/en-us/collaboration/fourthparadigm/

Hicks, D., Wouters, P, Waltman, L, De Rijcke, S, and Rafols, I 2015. 'Bibliometrics: The Leiden Manifesto for research metrics'. Nature, 520: 429-431.

Hobsbawm, $\mathrm{E}$ and Ranger, $\mathrm{T}$ (eds) (1983). The Invention of Tradition. Cambridge: Cambridge University Press.

Hojem, TSM (2012). 'Bridging two worlds? The troubled transfer of new environmental knowledge from science to consulting engineers'. Acta Sociologica, 55(4), 321-334.

Holbrook, J, Gu, DY, and Traweek, S (2018). 'Reaching for the stars without an invitation'. American Scientist, 106(5): 296-297.

Holbrook, J (2021). 'ASTROMOVES: Astrophysics, diversity, mobility'. arXiv preprint arXiv: 2101.10826.

Hood, C (1995). 'The "new public management" in the 1980s: Variations on a theme'. Accounting, Organizations and Society, 20(2-3): 93-109.

Hüther, O and Krücken, G (2016). 'Nested organizational fields: Isomorphism and differentiation among European universities'. Research in the Sociology of Organizations, 46: 53-83.

Ingelsrud, MH and Falkum, E (2019). Frie yrker, lite medbestemmelse? Medlemsundersøkelse av medvirkning og medbestemmelse blant Forskerforbundets medlemmer i statlig tariffområde høsten 2018. Report 2019:02, Oslo: Work Life Institute. 
Johnson PA, Widnall, SE, and Benya, FF (eds). (2018). Sexual Harassment of Women. Climate, Culture, and Consequences in Academic Sciences, Engineering, and Medicine. Washington DC: The National Academies Press.

Johnson, T (1972). Professions and Power. London: The Macmillan Press.

Kaiser, D (2002). 'Scientific manpower, Cold War requisitions, and the production of American physicists after World War II'. Historical Studies in the Physical and Biological Sciences 33: 131-159.

Kaloudis, A, Aspelund, A, Koch, PM, Lauvås, TM, Mathisen, MT, Strand, Ø, Sørheim, R, and Aadland, T. (2019). How Universities Contribute to Innovation: A Literature Review-based Analysis. Trondheim: NTNU. Available from www.ntnu.no/ documents/1272503658/1276139892/Rapport_How+universities+contribute+to+ innovation_web.pdf/2233414b-d9cd-85ad-6bc8-58b9bc0c9d78?t=1574847934147

Klein, JT (1990). Interdisciplinarity: History, Theory, and Practice. Detroit: Wayne State University Press.

Knorr-Cetina, K (1995). 'Laboratory studies: The cultural approach to the study of science'. In: Jasanoff, S, Markle, GE, Peterson, JC, and Pinch, T (eds). Handbook of Science and Technology Studies. Thousand Oaks: Sage, pp. 140-167.

Kristensen, A. 2020. 'Kartlegging av arbeidslivserfaring integrert i utdanningsløpet ved NTNU'. Unpublished working paper. Trondheim: NTNU, Department of Interdisciplinary studies of culture.

Krücken, G and Meier, F (2006). 'Turning the university into an organizational actor'. In: Drori, GS, Meyer, JW, and Hwang, H (eds.). Globalization and Organization: World Society and Organizational Change. Oxford: Oxford University Press, pp. 241-257.

Kuratko, DF and Morris, MH (2018). 'Corporate entrepreneurship: A critical challenge for educators and researchers'. Entrepreneurship Education and Pedagogy, 1 (1): 42-60.

Lagesen, VA (2021). 'How heads of departments find it meaningful to engage with gender balance policies'. Science and Public Policy, 48(4): 582-591.

Latour, B (1987). Science in action. How to follow scientists and engineers through society. Milton Keynes: Open University Press.

Latour, B (2004). Politics of Nature. Cambridge, MA: Harvard University Press.

LeBaron, C (1981). Gentle Vengence: An Account of the First Year at Harvard Medical School. New York: Marek.

Leydesdorff, L, and Milojević, S, 2015. 'Scientometrics'. In: JD Wright (ed). International Encyclopedia of Social and Behavioral Sciences, Oxford: Elsevier, pp. 322-327.

Lu, TJ (2015). 'UCLA senior management growth in comparison to faculty and students (1993-2014)' at https://scaleatucla.weebly.com/ucla-senior-management-gro wth-in-comparison-to-faculty-and-students-1993-2014.html (accessed June 23, 2021)

Lu, TJ (2021). 'Employee data tables in the University of California (1964-2015)' at https://doi.org/10.17605/OSF.IO/BCZQ4 (accessed June 23, 2021).

Marcus, GE (1995). 'Ethnography in/of the world system: The emergence of multisited ethnography'. Annual Review of Anthropology, 24(1): 95-117.

Marcuse, H (1964). One-dimensional Man: Studies in the Ideology of Advanced Industrial Society. Boston: Beacon Press.

Macfarlane, B (2007). 'Defining and rewarding academic citizenship: The implications for university promotions policy'. Journal of Higher Education Policy and Management, 29(3): 261-273. 
Maassen, P, Gornitzka, Å, and Fumasoli, T (2017). 'University reform and institutional autonomy: A framework for analysing the living autonomy'. Higher Education Quarterly, 71(3): 239-250.

Martin, E (2009). Bipolar Expeditions. Princeton University Press.

McAlphine, L and Amundsen, C (2018). Identity-Trajectories of the Early Career Researchers: Unpacking the Post-PhD Experience. London: Palgrave Macmillan.

McAlphine, L and Åkerlind, GS (eds). (2010). Becoming an Academic. International Perspectives. Houndmills, Basingstoke: Palgrave Macmillan.

McClure, KR (2014). Beyond Academic Capitalism: Innovation and Entrepreneurship as Institutional Ethos at a Public Research University. Diss. University of Maryland, College Park.

McClure, KR (2016). 'Building the innovative and entrepreneurial university: An institutional case study of administrative academic capitalism'. The Journal of Higher Education 87(4): 516-543.

McClure, KR (2017). 'Arbiters of effectiveness and efficiency: the frames and strategies of management consulting firms in US higher education reform'. Journal of Higher Education Policy and Management 39(5): 575-589.

Meld. St. 16 (2016-2017) Kultur for kvalitet i høyere utdanning (Culture for quality in Higher Education). www.regjeringen.no/contentassets/aee30e4b7d3241d5bd89db6 9fe38f7ba/no/pdfs/stm201620170016000dddpdfs.pdf (accessed June 20, 2021).

Meyerson, J and McClenon, C (2017, April 7), 'Campus computing cultures, mainframe retirement and meaningful reuse', Maintainers II, Stevens Institute of Technology at https://themaintainers.org/maintainers-ii-2017/ (accessed September 22, 2021).

Muller, JZ (2018). The Tyranny of Metrics. Princeton: Princeton University Press.

Müller, R and de Rijcke, S (2017). 'Exploring the epistemic impacts of academic performance indicators in the life sciences'. Research Evaluation, 26(3): 157-168.

Münch, R (2014). Academic Capitalism: Universities in the Global Struggle for Excellence. New York: Routledge.

Murillo, LFR, Gu, D, Guillen, R, Holbrook, J, and Traweek, S (2012). 'Partial perspectives in astronomy: Gender, ethnicity, nationality and meshworks in building images of the universe and social worlds'. Interdisciplinary Science Reviews, 37(1): 36-50.

Musselin, C (2007). 'Are universities specific organisations?' In: Krücken, G, Kosmützky, A, and Torka, M Towards a Multiversity? Universities Between Global Trends and National Traditions. Bielefeld: Transcript Verlag, pp. 63-84.

Musselin, C (2008). 'Towards a sociology of academic work'. In: Alberto, A, Bleiklie, I, and Musselin, C (eds). From Governance to Identity. A Festschrift for Mary Henkel, Dordrecht: Springer, pp. 47-56.

Musselin, C (2011). 'The academic workplace: What we already know, what we still do not know, and what we would like to know'. In: Rhoten, D and Calhoun, C (eds). Knowledge Matters: The Public Mission of the Research University, New York: Columbia University Press, pp. 423-456.

Newfield, C (2016). The Great Mistake: How We Wrecked Public Universities and How We Can Fix Them. Baltimore: Johns Hopkins University Press.

NOKUT. 2020. Evaluering av arbeidslivsrelevans i disiplinutdanninger. Report 4-2020. https://www.nokut.no/globalassets/nokut/rapporter/ua/2020/evaluering-avarbeidslivsrelevans-i-disiplinutdanninger_4-2020.pdf (Accessed October 3, 2021).

Nowotny, H, Scott, P, and Gibbons, M. (2001). Re-thinking Science: Knowledge and the Public in an Age of Uncertainty. Cambridge: Polity Press. 
OECD (2020). 'Addressing societal challenges using transdisciplinary research'. OECD Science, Technology and Industry Policy papers, no. 88. Paris: OECD.

OECD (2021). 'Reducing the precarity of academic research careers'. OECD Science, Technology and Industry Policy papers, no. 113. Paris: OECD.

Olsen, JP and Maassen, P (2007). 'European debates on the knowledge institution: The modernization of the university at the European level'. In: Maassen, P and Olsen, JP (eds) University dynamics and European integration. Dordrecht: Springer, pp. 3-22.

Ong, MT (2002). Against the Current: Women of Color Succeeding in Physics. PhD dissertation. University of California, Berkeley.

Ong, MT (2005). 'Body projects of young women of color in physics: Intersections of gender, race, and science'. Social Problems, 52(4): 593-617.

Ong, M, Smith, JM, and Ko, LT (2018). 'Counterspaces for women of color in STEM higher education: Marginal and central spaces for persistence and success'. Journal of Research in Science Teaching, 55(2): 206-245.

Ortiz, A (1965). 'Dual organization as an operational concept in the Pueblo Southwest'. Ethnology, 4(4): 389-396.

Ortiz, A (1969) The Tewa World: Space, Time, Being and Becoming in a Pueblo Society. Chicago: University of Chicago Press.

Pandey, TN (1967). Factionalism in a Southwestern Pueblo (Dissertation, University of Chicago), pp. 231-236.

Pandey, TN (1968). 'Tribal council elections in a Southwestern Pueblo'. Ethnology 7(1): 71-85.

Paradeise, C and Thoenig, J-C (2013). 'Academic institutions in search of quality: Local orders and global standards'. Organization studies, 34(2): 189-218.

Park, SJ, Lim, SY, and Park, HW (2015). 'Comparing Twitter and YouTube networks in information diffusion: The case of the "Occupy Wall Street" movement'. Technological Forecasting and Social Change, 95: 208-217.

Pereira, MdM (2017). Power, Knowledge and Feminist Scholarship: An Ethnography of Academia. London: Routledge.

Petersen, AH (21 July 2021). 'The Master's Trap: What makes a graduate program predatory?' Culture Study (accessed 25 July 2021) https://annehelen.substack.com/p/themasters-trap?token=eyJ1c2VyX21kIjo2NzY0ODk4LCJwb3N0X21kIjozOTAwOTE0 MiwiXyI6IlZBTHNTIiwiaWF0IjoxNjI3MjU5MjMwLCJleHAiOjE2MjcyNjI4MzAs ImlzcyI6InB1Yi0yNDUwIiwic3ViljoicG9zdC1yZWFjdGlvbiJ9.8PMFGxPxBEjacM TeNS2Zb_FeU0pqaRZ0zZx7ahyw6Ko

Pettersson, H (2016). 'Research cooperation, learning processes, and trust among plant scientists: Fictive kinship, academic mobility, and scientists' careers'. In: Domesticity in the Making of Modern Science (pp. 241-258). London: Palgrave Macmillan.

Petrou, P, van der Linden, D, Mainemelis, C, and Salcescu, OC (2020). 'Rebel with a cause: When does employee rebelliousness relate to creativity?' Journal of Occupational and Organizational Psychology, 93(4): 811-833.

Pfotenhauer, SM, Juhl, J, and Aarden, E (2019). "Challenging the "deficit model" of innovation: Framing policy issues under the innovation imperative'. Research Policy, 48(4), 895-904.

Phan, PH, Wright, M, Ucbasaran, D, and Tan, WL (2009). 'Corporate entrepreneurship: Current research and future directions'. Journal of Business Venturing, 24 (3): 197-205.

Pielke, R (2014). 'In retrospect: The social function of science'. Nature 507: 427-428. 
Pirsig, RM (1974) Zen and the Art of Motorcycle Maintenance: An Inquiry into Values. New York: William Morrow.

Polanyi, M (1962) 'The republic of science: Its political and economic theory'. Minerva, 1(1): 54-73.

Proctor, RN and Schiebinger, L (eds). (2008). Agnotology: The Making and Unmaking of Ignorance. Stanford: Stanford University Press.

van Raan, AFJ (2005). 'Fatal attraction: Conceptual and methodological problems in the ranking of universities by bibliometric methods'. Scientometrics, 62(1): 133-143.

Readings, B (1996). The University in Ruins. Cambridge: Harvard University Press.

Reichman, H (2019). The Future of Academic Freedom. Baltimore: Johns Hopkins University Press.

Rhodes, GHT (2001). The Creation of the Future: The Role of the American University. Ithaca: Cornell University Press.

Robin, C (2020). 'The Pandemic is the time to resurrect the public university'. New Yorker 7 May, Available from https://www.newyorker.com/culture/ cultural-comment/the-pandemic-is-the-time-to-resurrect-the-public-university.

van Raan, AFJ (2005). 'Fatal attraction: Conceptual and methodological problems in the ranking of universities by bibliometric methods'. Scientometrics, 62(1): 133-143.

Rosenzweig, P (2010). 'Robert S. McNamara and the evolution of modern management'. Harvard Business Review, 88 (12): 87-93.

Sahlin, K and Eriksson-Zetterquist, U (2016). 'Collegiality in modern universities: The composition of governance ideals and practices'. Nordic Journal of Studies in Educational Policy, (2-3): 33640.

Sarewitz, D (2016). 'Saving science'. The New Atlantis, 49(Spring/Summer): 4-40.

Sarrico, C, McQueen, A, and Samuelson, S. (eds). (2017). State of Higher Education 2015-16. Paris: OECD.

Schendel, D (1990). 'Introduction to the special issue on corporate entrepreneurship'. Strategic Management Journal, 11 (Summer): 1-3.

Scott, JW (2019). Knowledge, Power, and Academic Freedom. New York: Columbia University Press.

Scott, P (2017). 'A third way for Europe's higher education system: Between American and Asian dreams and realities'. In: Austrian Council for Research and Technology Development (ed.) Prospects and future tasks of universities. Vienna: Lit Verlag, pp. 273-290.

Seglen, PO (1997). 'Why the impact factor of journals should not be used for evaluating research'. British Medical Journal, 314: 498-502.

Shapin, S (2012). 'The ivory tower: The history of a figure of speech and its cultural uses'. The British Journal for the History of Science, 45(1): 1-27.

Showalter, E (2005). Faculty Towers: The Academic Novel and Its Discontents. Oxford: Oxford University Press.

Slaughter, S and LL Leslie (1997). Academic Capitalism. Politics, Policies, and the Entrepreneurial University. Baltimore: Johns Hopkins University Press.

Slaughter, S and G Rhoades (2004). Academic Capitalism and the New Economy. Markets, State, and Higher Education. Baltimore: Johns Hopkins University Press, 2004.

Smith-Doerr, L (2004). 'Flexibility and fairness: Effects of the network form of organization on gender equity in life science careers'. Sociological Perspectives, 47(1): $25-54$.

Smith-Doerr, L (2005). 'Institutionalizing the network form: How life scientists legitimate work in the biotechnology industry'. Sociological Forum 20(2): 271-99. 
Staley, DJ (2019). Alternative Universities. Speculative Design for Innovation in Higher Education. Baltimore: Johns Hopkins University Press.

Standards and Guidelines for Quality Assurance in the European Higher Education Area (ESG). (2015). Brussels, Belgium.

Stilgoe, J, Owen, R, and Macnaghten, P (2013). 'Developing a framework for responsible innovation'. Research Policy, 42(9): 1568-1580.

Stone, T (2009) 'Departments in academic receivership: Possible causes and solutions'. Innovative Higher Education. 33: 229-238.

Strathern, M (1997). "Improving ratings": Audit in the British university system'. European Review, 5(3): 305-321.

Strathern, M (2000). (ed). Audit Cultures: Anthropological Studies in Accountability, Ethics and the Academy. London: Routledge.

Suchman, L (2008). 'Feminist STS and the sciences of the artificial'. In: Hackett, EJ, Amsterdamska, O, Lynch, M, and Wajcman, J (eds). The Handbook of Science and Technology Studies, Cambridge, MA: MIT Press, pp. 139-163.

Svalund, J, Andersen, RK, Bråten, M, and Hagen, IM (2020). 'Ansattes opplevelse av medvirkning og medbestemmelse ved NTNU'. Fafo-rapport, 2020:22, Oslo: Fafo.

Sørensen, KH (2006). 'Domestication: The enactment of technology'. In T Berker, M Hartmann, Y Punie, and KJ Ward (eds). Domestication Revisited. Maidenhead: Open University Press, pp. 40-61.

Sørensen KH (2019) " "Mas, mas, mas - over hele linja”: Om disiplineringen av universitetene til "samfunnsoppdraget". In: Tjora, A (ed). Universitetskamp. Oslo: Scandinavian Academic Press, pp. 450-483.

Sørensen, KH and Moratti, S (2019). 'The publication culture at the Faculty of Humanities, NTNU'. A survey. Available from https://ntnuopen.ntnu.no/ntnuxmlui/bitstream/handle/11250/2624342/publ+cult+final.pdf?sequence $=1$

Thorsrud, E and Emery, FE (1970). 'Industrial democracy in Norway'. Industrial Relations: A Journal of Economy and Society, 9(2): 187-196.

Tjeldvoll, A (1998). 'The service university in the global marketplace'. European Education, 30(4): 5-19.

Traweek, S (1988). Beamtimes and Lifetimes the World of High Energy Physicists. Cambridge, MA: Harvard University Press.

Traweek, S (2000). 'Faultlines'. In: Reid, R and Traweek, S (eds). Doing Science \& Culture. New York: Routledge, pp. 21-48.

Traweek, S (2005). 'Generating high energy physics in Japan: Moral imperatives of a future pluperfect'. In: Kaiser, D (ed), Pedagogy and the Practice of Science: Historical and Contemporary Perspectives, Cambridge, MA: MIT Press, pp. 357-392.

Traweek, S (2021a). 'Let anthropology burn?' Anthropology Now, 12(3): 34-38.

Traweek, S (2021b). 'I Prefer the map'. Engaging Science, Technology, and Society. Vol 7.

Traweek, S (forthcoming, 2022). 'Making Difference at the Edge'. In: S Harding and

L Rodriguez (eds), Decentralizing Knowledges: Essays on Distributed Agencies, Medina: Duke University Press.

Turow, S (1977). One L: An Insider's Account of Life in The First Year at Harvard Law School. New York: Putnam.

Usher, A and Savino, M (2007) 'A global survey of rankings and league tables'. In: Sanoff, AP, Usher, A, Savino, M, and Clarke, M (eds), College and University Ranking Systems. Global Perspectives and American Challenges, Washington, DC: Institute for Higher Education Policy, pp. 9-34.

Vabø, A and Ramberg, I (2009). Arbeidsvilkår i norsk forskning, rapport 9/2009. Oslo: Nordic Institute for Studies in Innovation, Research and Education. 
Venters, W and Wood, B (2007). 'Degenerative structures that inhibit the emergence of communities of practice: a case study of knowledge management in the British Council'. Information Systems Journal, 17(4): 349-368.

Vertesi, J (2020). Shaping science: Organizations, decisions, and culture on NASA's teams. Chicago: University of Chicago Press.

Weber, M (2020). Charisma and Disenchantment. The Vocation Lectures. New York: New York Review Books.

Weingart, P and Massen, S (2007). 'Elite through rankings: The emergence of the enterprising university'. In: Whitley, R and Gläser, J (eds). The Changing Governance of the Sciences, Dordrecht: Springer, pp. 75-99.

Weingart, P and Stehr, N (eds). (2000). Practising Interdisciplinarity. Toronto: Toronto University Press.

White, P (1991). The Idea Factory: Learning to Think at MIT. Boston: Dutton.

Whitley, R (2008). 'Construction universities as strategic actors: Limitations and variations'. In: Engwall, L and Weaire, D (eds). The University in the Market. London: Portland Press, pp. 23-37.

Whitley, R (2012). 'Transforming universities: National conditions of their varied organisational actorhood'. Minerva, 50(4), 493-510.

Williams, JJ (2012). 'Deconstructing academe. The birth of critical university studies'. Chronicle of Higher Education, 58(25): B7-B8.

Williams, JJ (2016). 'The need for critical university studies'. In Hutner, G and Mohamed, FG (eds). A New Deal for the humanities: Liberal arts and the future of public higher education, Chicago: Rutgers University Press, pp. 145-59.

Williamson, B (2018). 'The hidden architecture of higher education: Building a big data infrastructure for the "smarter university"'. International Journal of Educational Technology in Higher Education, 15(1): 1-26.

Wilsdon, J et al. (2015). The Metric Tide. Report of the Independent Review of the Role of Metrics in Research Assessment and Management. Accessible at https:// responsiblemetrics.org/wp-content/uploads/2019/02/2015_metrictide.pdf

Wittrock, B (1993). 'The modern university: The three transformations'. In: Rothblatt, S and Wittrock, B (eds). The European and American University Since 1800: Historical and Sociological Essays, Cambridge: Cambridge University Press, pp. 303-362.

Wolcott, RC and Lippitz, MJ (2007). 'The four models of corporate entrepreneurship'. MIT Sloan Management Review, 49 (1): 75-82.

Wylie, A (2012). 'Feminist philosophy of science: Standpoint matters'. Proceedings and Addresses of the American Philosophical Association. 86(2): 47-76.

Zorn, D and Boler, M (2007). 'Rethinking emotions and educational leadership'. International Journal of Leadership in Education, 10(2): 137-151. 


\section{Name Index}

Aarden, E 213

Aberbach, JD 39, 206

Ainsworth, T 208

Albert, M 177, 209

American Association of University

Professors [AAUP] 206

Amundsen, C 155, 212

Andersen, RK 215

Anderson, BR 13, 206

Anzaldua, G 95

Arendt, H 206

Ashmore, M 20, 206

Ashmore R 206

Aspelund, A 211

Baldwin, RG 146, 206

Bates, AE 208

Bateson, G 34-35, 109, 137, 205-206

Baum, JK 208

Bauman, Z 206

Bear, L 13, 206

Beck, U 52, 206

Becker, P 157, 207

Belfiore, E 216

Benya, FF 211

Berman, EP 13, 24, 40, 207

Bernal, JD 200-201, 207

Bleiklie, I 207, 212

Boler, M 145, 216

Bos, C 85, 207

Bourdieu, P 8, 129, 207

Bove, CB 208

Bråten, M 215

Brundtland, GH 33, 184, 207

Brunsson, N 6, 207

Burgelman, RA 111, 207

Burrows, R 14, 207
Calhoun, C 10-11, 207, 212

Chatterjee, P 204, 207

Christensen, T 39, 170, 206-207

Chronister, JL 146, 206

Clark, BR 12-13, 122, 158, 186, 207

Clark, W 5, 46, 81, 109, 207

Clarke, M 215

Collini, S 2-3, 14, 36, 186, 207

Collins, HM 55, 207

Collins, PH 25, 112, 207

Connell, R 2-3, 14, 16, 26, 207

Coser, LA 207

Covin, JG 111, 207

Crosby, SC 208

Crow, MM 11, 13, 207

Cummings, WK 161, 186, 207

Czarniawska, B 7, 207

Daenekindt, S 16, 208

Dahling, JJ 145, 191, 208

Davies, SR 43, 208

Davies, SW 45, 208

Dabar, WB 11, 13, 207

Deleuze, G 113, 208

Derrida, J 95

DiMaggio, PJ 3, 208

Doing, P 104, 208

Donoghue, F 2, 14, 208

Douglass, JA 11, 13, 35, 186, 208

Drucker, P 50, 85, 208

Dubinskas, FA 157, 193, 208

Daugstad, K 176, 208

Elken, M 163, 208

Emery, FE 96, 215

Eriksson-Zetterquist, U 8, 80, 97, 214

Espeland, WN 45, 208 
Etzkowitz, H 17, 208

Evans, M 2, 14, 208

Falkum, E 82, 210

Fanon, F 112, 208

Felt, U 111-112, 156, 209

Fleming, P 209

Fleming, P 2-3, 14, 36, 112, 186, 209

Fochler, M 112, 156, 187, 209

Foucault, M 85, 113, 209

Fortun, K 20, 200, 209

Fortun, M 20, 200, 209

Frank, DJ 1, 81, 209

Frickel, S 177, 209

Frodeman, R 177, 209

Fromm, E 209

Fumasoli, T 39, 211

Furstenberg, F 209

Gabler, J 1, 209

Gibbons, M 18, 179, 212

Gill, RC 3, 112, 209

Gingras, Y 17, 209

Godin, B 17, 209

Goetzmann, WN 203, 209

Gonzalez, S 210

Gornitzka, Å 39, 71, 209, 211

Gu, DY 144, 209-210, 212

Guattari, F 113, 208

Guillen, R 212

Gumport, PJ 16, 158, 209

Gunnes, H 209

Gusterson, H 19, 203, 210

Gutworth, MB 145, 191, 208

Hackett, EJ 14, 16, 210, 215

Hagen, IM 215

Halffman, W 2, 14, 210

Haraway, D 20, 25, 112, 138, 210

Hardin, G 161, 210

Harding, S 20, 25, 112, 206, 210 , 215

Hartsock, N 20, 25, 210

Henry, FW 157, 210

Heracleous, L 145, 191, 210

Herrington, G 205, 210

Hey, T 116, 210

Hicks, D 45, 57, 210

Hobsbawm, E 121, 210

Holbrook, JB 209

Holbrook, JC 144, 210, 212

Hood, C 6, 210
Huisman, J 16, 208

Hüther, O 3, 210

Igelsböck, J 209

Ingeborgrud, LH 183

Ingelsrud, MH 82, 210

Johnson, PA 211

Johnson, T 166, 211

Juhl, J 213

Kaiser, D 59, 211, 215

Kaloudis, A 176, 211

Klein, JT 177, 209, 211

Knorr-Cetina, K 17, 211

Kristensen, A 166

Krücken, G 3, 6, 44, 210-212

Kuratko, DF 111, 211

Kusch, M 55, 207

Kyvik, S 71, 209

Larsen, IM 71, 209

Lagesen, VA 50, 53, 211

Latour, B 18, 90, 211

LeBaron, C 157, 211

van Lente, $\mathrm{H} 207$

Leslie, LL 40, 214

Leydesdorff, L 17, 40, 208, 221

Lim, SY 86, 213

van der Linden, D 213

Lippitz, MJ 111, 191, 216

Maassen, P 39, 211, 213

Macfarlane, B 101, 211

Macnaghten, P 8, 215

Mainemelis, C 213

Martin, E 20, 212

Marcus, GE 9, 211

Marcuse, H 99, 211

Massen, S 11, 216

Marx, K 95, 156, 200

Maira, S 204, 207

Mathur, N 13, 206

McAlphine, L 155, 212

McQueen, A 214

Meier, F 6, 211

Meld. St. 47, 168, 212

Meyer, JW 1, 44, 81, 209, 211

Miles, MP 111, 207

Milojević, S 211

Mitcham, C 209

Moratti, S 96, 107, 215 
Morris, MH 111, 211

Muller, JZ 40, 212

Müller, R 189, 212

Münch, R 14-15, 212

Murillo, LFR 144, 212

Musselin, C 6-7, 16, 212

Newfield, C 14, 212

Nowotny, H 18, 178, 212

NOKUT 27-28, 166

OECD 111, 158, 177, 179, 212

Olsen, JP 213

Ong, MT 121, 143, 213

Ortiz, A 2, 213

Oster, S 203, 209

Owen, R 8, 215

Pandey, TN 87, 213

Paradeise, C 3, 207, 213

Park, HW 86, 213

Park, SJ 86, 213

Peine, A 207

Pereira, MdM 112, 213

Petersen, AH 202, 213

Pettersson, H 77, 213

Petrou, P 145, 191, 213

Pfotenhauer, SM 173, 201, 213

Phan, PH 111, 213

Pielke, R 200, 213

Pirsig, RM 55, 213

Polanyi, M 200, 213

Powell, WW 3, 208

Prainsack, B 177, 209

Proctor, RN 22, 204, 214

Putnam, HM 208

van Raan, AFJ 45, 214

Radder, H 2, 14, 210

Ramberg, I 215

Ranger, T 121, 210

Readings, B. 14, 165, 214

Reichman, H 78, 214

Rhodes, GHT 2, 14, 214

de Rijcke, S 209, 212

Robin, C 65, 214

Robson, D 145, 210

Rosenzweig, P 41, 214

Sahlin, K 7-8, 80, 97, 214

Sahlin-Andersson, K 6, 207

Salcescu, OC 213
Samuelson, S 214

Sandoval, C 95

Sarewitz, D 189, 214

Sarrico, C 214

Sauder, M 45, 208

Savino, M 44, 215

Schendel, D 111, 214

Schiebinger, L 22, 204, 214

Schikowitz, A 209

Scott, JW 78-79, 98, 214

Scott, P 3, 18, 179, 212, 214

Seglen, PO 45, 214

Showalter, E 110, 214

Slaughter, S 14, 40, 214

Smith-Doerr, L 214

Solbu, G 206

Staley, DJ 186, 214

Stehr, N. 177, 216

Stilgoe, J 8, 215

Stone, T 90, 215

Strathern, M 56-57, 181, 187, 215

Suchman, L 55, 215

Svalund, J, 215

Sørensen, KH 13, 41, 47, 50, 96, 107, 206, 215

Tan, WL 213

Tansley, S 116, 210

Terrier, D 210

Thoenig, J-C 3, 46, 213

Thorsrud, E 96, 215

Tjeldvoll, A 215

Tolle, K 116, 210

Traweek, S 5, 17, 32, 110, 121, 144, 157, 208, 210, 212, 215

Turow, S 157, 215

Ucbasaran, D 213

Usher, A 44, 215

Vabø, A 215

Venters, W 86, 215

Völker, T 209

Vertesi, J 20, 216

Walhout, B 207

Weber, M 99, 100, 216

Weingart, P 177, 209, 216

Wouters, P 216

White, H 138

White, P 157, 216

Whitley, R 6, 216 
Widnall, SE 211

Williams, JJ 13, 216

Wilsdon, J 40, 216

Wittrock, B 5, 216

Wolcott, RC 111, 191, 216

Wollscheid, S 163, 208

Wood, B 86, 215
Wright, M 211, 212

Wylie, A 25, 216

Zorn, D 145, 216

Åkerlind, GS 109, 212

Åm, H 8, 155, 172, 206 


\section{Keyword Index}

academic capitalism 1, 14-15, 33, 36, 40, 69, 73, 105-106, 186, 210, 212, 214 see corporatization; entrepreneurs and entrepreneurship; neoliberal; political economy

academic citizenship 33, 72, 101-104, 109, 152, 155, 167, 185, 189, 205, 211 see academic freedom, autonomy, bureaucratizing, commons, corporatizing, governance academic freedom 2-4, 7, 33, 36, 38, $50,54,66,72-73,78-80,83,87,90$, 95, 97-101, 104-106, 151, 169, 186, 193, 214 see governance administrators 2, 26, 34, 39, 42, 56, $64,71,76,86,88-89,128,160,188$, 192-197, 199, 204-205 see academic citizenship; faculty members [professoriate]; staff, students algorithm 55-56, 64-65, 106, 142-143; algorithmic governance 56, 62, 65, 99 , 105, 145; corporate algorithmists 105 see assessment, budget, domesticating, factions, software platforms assessment 7, 12, 14-15, 24, 33, 39-41, 44-46, 48-51, 56-58, 65-66, 72, 75, $79,81,84,86,99-101,103-104$, 109-111, 114, 149, 152-154, 156, 164-166, 169, 174, 187-190, 200, 216 see governance; software platforms autoethnography 20-21, 92, 113 see cultures, ethnography; fieldwork; see also participant observation autonomy: academic and faculty $7-8$, 79-80, 87, 94-101, 106, 147, 155, 193, 206; autonomy of universities 33, 37-39, 54, 66, 79-80, 184, 193, 207.
211; local and group 66, 69, 72-73, 211; see corporatization

branding $37,40,42-44,46,56,106,116$; brand $30,43-44,111,116$

budget $30,44,47,51,54-55,62,70-71$, $74,81,83,85-86,90,106,131,141$, $156,179,191$; incentives 69 ; indicators 51; management 142; model 27, 49-50, 53, 61-62, 188; objectives 49; planning 57 ; system 47,66

bureaucratizing 33-34, 40-41, 47-53, 66, 71-72, 80-81, 83-84, 96, 98, 104, 108, 111-112, 184, 187, 190-191,

193, 199, 201-202; bureaucratic 2, 5, 13, 28, 34, 41, 49-50, 74, 79, 82, 84, 98-99, 102, 104, 116, 126, 148, 151, 169, 175-176, 184, 190-193, 204, 207; bureaucratization 14, 28, 33, 41, 51, 169 see corporatizing; governance

California 2, 9, 28-31, 34, 46, 52, 54, 60-62, 64, 66, 71, 78-79, 83, 105-106, 117-118, 131-132, 136-137, 141, 160, $173,175,191,204,208,211,213$

careers 16, 21, 23, 41, 76, 99-100, 109, 113-115, 117, 134-135, 138, 141-142, 144, 146, 149, 152, 155-156, 168, 170-171, 180, 192, 212 see subject formation

categories [categorical] 21, 60, 65, 126, 174

collegial organizing $7-8,13,15,33,72$, 80, 83-84, 87, 90, 95-97; 99, 101, 194, 199; systems $8,15,80,101$ collegiality $7,19,69,72,80,87,95,214$; 'collegial' 80 ; practices 7, 12, 72, 79-82, 87, 90, 101-102, 110, 153; relations 
7-8, 66, 78, 95, 104, 184, 188; see also collaboration

commons 34, 160-162, 192, 198, 201, 203, 205, 210; knowledge commons 161-162, 172, 175, 177, 182, 201, 203 see pawa hara; see also borderlands, crossroads, margins, mixtures

co-morphing and re-morphing 7, 22-25, $34,65,192$, 204-205; morphing 50, 85 see domesticating, meshworking corporatizing 33-34, 40, 47, 54-66, 69, $72,80,83-87,96,98,104,109,113$, 177, 184, 187, 190-193, 199, 201-202, 204-205 see bureaucratizing cultures 2-4, 6, 9-10, 12-14, 16-17, 19, 23-25, 30, 42, 52, 57, 63-66, 72-73, $77,80-81,91,97,103,105,109-110$, $113,127,129,136,146-147,150-151$, $154,160,166,168,175-176,180$, 184-185, 187, 192-194, 198-199, 203, 206, 209-216; whisper 127, 198; see also ranked, regional, occupational

disciplines 19, 34, 39, 45, 73, 91, 95, 102, $108,113,133,144,148-150,168$, 177-182, 185, 188, 199, 201, 209

doctorate $58,60,67,110,117-118,121$, 124, 128, 136-137, 146-147, 171, 179,202

domesticating 7, 31, 34, 192, 205; domesticate 24, 31, 41, 50, 163; domestication 41, 50-51, 204, 215

entrepreneurs and entrepreneurship 105, 108, 110-112, 114, 120, 123, 131, 134, $137,141,145,156,160,173-175$, 191-192, 207, 211-213 see innovation; see also outreach

epistemic 7, 19, 62, 65, 99, 105, 123, 135, 143-144, 156, 161, 192, 199, 202, 204, 209, 212; angst 177-181; authority 2, 19, 26, 162, 190, 199; choices 113-117 see subject formation epistemic politics $3-4,7-8,12-13,15$, $17-18,23,38,72,78-81,95-96$, 104-105, 109, 112, 171, 178, 180, 185, 187, 194, 200, 208

epistemic practices 3-4, 8, 12-13, 15-20, 23, 38, 116; see also knowledge making

ethnography 9, 19, 210-211, 213 see autoethnography; cultures; fieldwork evaluations $21,28,34,41,53,55-58$, 62-63, 71, 74-75, 77, 97, 101, 114-115, 123, 130, 134-135, 139-146, 153-154, 156, 169, 174, 185, 187-190, 204, 212, 214; evaluate 3, 39, 58, 117 , 123, 139, 169, 189-191 see assessment; metrics

excellence 41-46, 49-50, 52-54, 56, 65-66, 71-72, 85, 92, 97, 99, 104, 112, 141-142, 145, 155-156, 170, 188, 190, 205, 212; excellent $10-11,42,47,52-53,65$, $95,108,125,140,144,188$; research excellence 4, 44, 53, 102-103, 162, 167; Research Excellence Framework (REF) 56-57 see corporatizing, metrics, quality

factions 33-34, 66, 72, 87-95, 104, 125, 192, 194, 196-198 see governance faculty members [professoriate] 17, 23, 34, 44, 51, 56, 58, 60-61, 64, 71, 74-77, 81-83, 85, 87-91, 94, 96-99, $101,103,108,114-119,121-128$, 130-138, 141, 146, 149-156, 158, 160, 162-164, 167-172, 178-179, 182, 187-188, 190, 192-197, 203, 205; professorship 87, 130, 133, 135 see administrators, students, staff, subject formation

faultlines $4,7,25,32,34,51,65,135$, 144, 162-163, 170, 182, 192, 199, 203-205, 215

field site: multi-sited 9, 16, 19, 23, 25

fieldwork 20, 34, 95 see autoethnography, ethnography, NTNU, UCLA see also participant-observer

funding $4,6,12-15,19,23-24,27$, 30-32, 36-38, 40-42, 44-46, 53, $56-57,60,62,75,80,85,87,89,92$, 94, 98, 108, 114-120, 126-131, 134, $136,139,146,150-151,154,158-159$, 167, 170-172, 177, 179-182, 191-192, 197, 201, 203 see academic capitalism, budget, entrepreneurship, governance, political economy, revenue streams

gossip 142, 196-199 see collegiality, governance, meshworks

governance 2, 5, 8, 13, 24-25, 28, 34, 36-38, 40-41, 45, 47-56, 62, 65, 69, 71-73, 78, 80-83, 88-90, 98-99, 105, $114,145,156,168-169,174,184-185$, 
188, 190-191, 193, 196-198, 204-207, 209, 212, 214, 216

government $3,5,9,12,16-17,19,27-28$, 30-33, 36-42, 44, 46-54, 56, 59, 61, 64-66, 69, 71, 75, 79, 81, 83, 86-88, 103, 106, 116, 119, 129-131, 136, 151, 155-156, 158-160, 168, 170-173, 176-177, 182, 185-188, 190-192, 202-203, 205, 208; governmentality $54,85,87,99,155$

harassment 4, 8, 70, 72, 90-91, 95-96, 102, 125, 127, 155, 194, 198-199, 202, 211; harassers 94, 198 see pawa hara; see also bullying

imagined university $13,51,85,121,155$, 193, 206, 215; imaginary 159, 161, 172; see also ideal academic indicators $12,15,22,40-41,44,49$, $51-53,57,61,65-66,69,90,120$, 173-174, 176-177, 186-190, 208-209, 212 see assessments, meritocracy

inequities 33, 102, 136, 159, 192, 203-205; see also precarity

infrastructures $23,25,29,56-57,71,74$, $76,87,99,105,120,127,140$, 144-145, 163, 179, 181, 192, 202, 216 innovation 1, 4-5, 8, 17, 24-25, 27, 33, 41, 47, 54, 70, 85, 98, 103, 109, 111, $141,146,156-159,162-163,172-177$, 181, 191-192, 201, 204, 206-209, 211-215 see entrepreneurs and entrepreneurship, research, teaching; see also outreach

interdisciplinarity $23,37,95,98,103$, 114, 121, 129-130, 132-133, 136, 147, 151, 163, 172, 177-182, 192, 199, 201, 205, 209, 212 see commons; see also borderlands, crossroads, margins, mixtures

juxtaposition 9-10, 24, 46, 66, 109, 136, 155-156, 190

mentors, mentoring 89, 92-94, 125-126, $129,137-139,142,145-146,148$, 152,155

meritocracy 7, 33, 72, 99-101, 109, 187 see academic citizenship, epistemic politics, subject formation meshworking 7, 24-25, 32-34, 76, 78, 81, $99,129,144,162-163,167,171-172$, 176, 181-182, 185, 193, 197-198, 200, 204-205, 212 see Interdisciplinarity metrics 15, 24, 33-34, 36-41, 44-47, 49, 51-52, 54-57, 61, 64-66, 69-71, 83, 88, 94, 99, 103-104, 106, 111, 117, $140,142-145,151,154-156,162$, 168-169, 173-174, 176, 182, 187-191, 202, 205, 207-208, 210, 212, 216

mobility 23-24, 34, 73, 76-77, 97, 134, 174, 176, 204, 210, 213

neoliberal university $2-6,8-9,12,15,22$, 24-25, 33, 37-38, 40-43, 46, 50-51, 56-57, 62, 65-66, 72, 96-98, 101, 103-104, 109, 127, 144, 155, 159, 161, 172, 181-182, 186, 190, 194, 199, 201-202, 204, 207, 209

Norway 9, 23, 26-28, 36, 45, 47-51, 53-54, 57, 75-78, 82-83, 96, 108-110, 146-156; work-life culture 96, 154, 166

NTNU 2, 9, 12-13, 19, 23, 26-28, 32-39, 42-54, 56-57, 61-62, 65-66, 69, 71-76, 79-90, 96-98, 100, 102-103, $106,109,111,113,147-148,150-151$, 154-156, 158-170, 172-177, 179-184, 186, 190-196, 199, 203-204, 208, 211-212, 215 see field site

pawa hara 91-95, 192, 198-199, 205 see harassment, subject formation; see also bullying

pedagogies $21,71,103,108,111,116$, 123-125, 134-135, 168-170, 215; pedagogical $78,96,103,163,168$, 202-203

political economy $3,9,13,19,25-26,28$, 32-34, 36-37, 42, 44, 46, 65-66, 80, $91,105,109,111-113,120,131,145$, 156, 190, 192, 194, 203

politics 4, 22, 52, 66, 75, 105, 108-109, 155, 186, 201, 214 see epistemic politics

practices $2-9,12-16,19-26,28,33-34$, 37-42, 50, 65-66, 73-74, 76-77, 80, 84-85, 87, 92, 95-97, 101-102, 108-111, 117, 121, 125, 127, 137, 141, $144,161,163,179,182,185,187-190$, 194, 199-201, 203-205; assessment practices $39,55-57,66,101,188-189$; collegial 5, 12, 15, 72, 79, 81-82, 87, 
99-101; communities of practice 86 , 143, 215; local practices 2, 37, 63, 70; governance practices $34,51,69$, 71, 73, 78, 81-82, 105, 184; metric management practices $3,38-41$, 61-62, 69-70, 82, 104; teaching and research practices $21,28,44,84,102$, 135, 137, 149, 166 see bureaucratizing, corporatizing, epistemic practices, meshworking

precarity $33,111,158,184,212$

private and public goods 5, 8-9, 12-14, 37-38, 42, 49, 158-159, 162 see research, teaching; see also outreach

quality $2,10-11,14-15,24-25,27-28$, $34,39,41-42,45,47-48,50-57,64$, $66,71,76,80,83-84,88,90,99-100$, 102-104, 106, 110, 113, 142, 147-149, $153,159,162-164,167-170,185$, 189-191, 200, 202, 204, 212-214

rankings $11,15,22,28,32,37,40,42$, $44-47,49,52-53,56-66,70,77,88$, 105-106, 108, 110-111, 120, 141, 144-145, 153, 155-156, 169, 175-176, 188-190, 203, 205, 208, 214-215 see assessments, branding, receivership 90-91, 215

regulating $36-37,42,54,62,103,106$, $126,154,167,170,193$

reputations $8,15,32-33,37,43-46,49$, $51,53,61,65-66,78,89,91,94,98$, $118,120,127,129,133,139,142,156$, 162, 208 see rankings

research 1-5, 92-93, 96, 115-117, 207-210, 212-216; applied 116, 128, 130, 151; bureaucratization 14-15, 84, 151; collaboration 101, 151; Declaration on Research Assessment (DORA) 188; ethics 8, 102; ethnographic 18-22; excellence 4, 44, 53-54, 56, 102-103, 162, 167; funding 30-32, 38, 42, 45-46, 54, 62, 85, 108, $114,118-120,129,131,134,146$, 170-172, 177-179, 192, 203; groups 17, 52-53, 74, 97, 151, 174; interdisciplinarity $129,172,177-178$, 181; methods 19, 63, 116, 122-123; Ministry of Education and Research 27, 52, 69, 103, 188; National
Research Council (NRC) 58; peer review 79, 101, 190, 203; policies 8, 12 , 52, 161, 164, 172, 177, 180, 200-201, 208-209, 213, 215; politicallyprioritized 47-48; professionalization of research 81,83 ; relationship to university ranking 44 46; Research Excellence Framework (REF) research university 5, 10, 109, 158, 207, 212; R1 60, 131-132, 139; researchbased innovation 54, 173-175, 18; RRI (Responsible Research and Innovation) 56-57; social relevance 10, 11, 19, 24, 66, 102-103, 190, 200; tension between excellence and accessibility 10 ; training $33,81,83$, 104, 109-110, 146-149; transforming research through teaching 17-18, 163-172 see private and public goods; see also outreach

revenue streams $38,64-65,106,108,110$, 160,203

self-management 7, 95, 97-97, 99, 101,193

software platforms $6,55,62-65,71$, 84, 191 see also CRM, HCM

STS [science, technology, and society/ science and technology studies] 1 , 16-17, 19-20, 23-26, 63, 115, 139-140, 151, 153-154, 161, 178-179, 187, 190, 202-204, 215

students 1, 4-5, 8, 10-11, 14, 16-17, 19, 22-23, 25-27, 33-34, 41-42, 44-46, 48-52, 56-65, 69, 71, 79, 81-82, 84, 87-91, 94, 97, 101-103, 108-109, 111, 135-139, 158-170, 184, 187, 191-192, 194-195, 202-203, 205, 211; graduate 74, 109, 121-129, 133, 137-138, 149-150, 156, 170; innovation 172, 174, 176, 179-181; normative-time-todegree 59, 119, 151; $\mathrm{PhD} 48,75,89$, 103, 147-152, 170; undergraduate 30-31, 63, 74, 77, 113-118, 146, 166, 168; see administrators, faculty members [professoriate], staff, subject formation

subject formation $21,23,25,33,62,72$, 104, 108-109, 111-113, 135, 144, 151, $155-156,187$

sustainable $32-33,47,86,102,146$, 184-185, 199, 201-22, 204-205 
teaching 1-2, 4-7, 10-11, 13-18, 23-25, 28, 33-34, 36-37, 41, 44, 47-48, 51, 53-58, 62-63, 65, 70, 74-75, 78-81, 84-85, 87-88, 90, 94, 96-104,

$110-111,118-119,124-125,128-129$, $138,142,145-146,149-150,153-154$, $156,159,162-170,171-177,180-182$, 185-186, 189, 191, 193, 201-203, 213 see research, innovation

UCLA 2, 9, 12-13, 19, 23, 26, 28-39, 42-46, 54-66, 69-73, 76-80, 82-84,
86-94, 97-98, 101-103, 105-106, 109-111, 113-115, 117, 121, 123, 127-128, 130, 132-133, 140-145, 155-156, 159-163, 166, 169, 171-176, 179, 183, 186, 190-197, 199, 203 see field site

University Studies 7, 13, 16, 146; critical university studies 7 , 13-15, 26, 34, 40, 57, 69, 80, 186-188, 216; higher education studies 23, 26, 34 see collegial organizing 


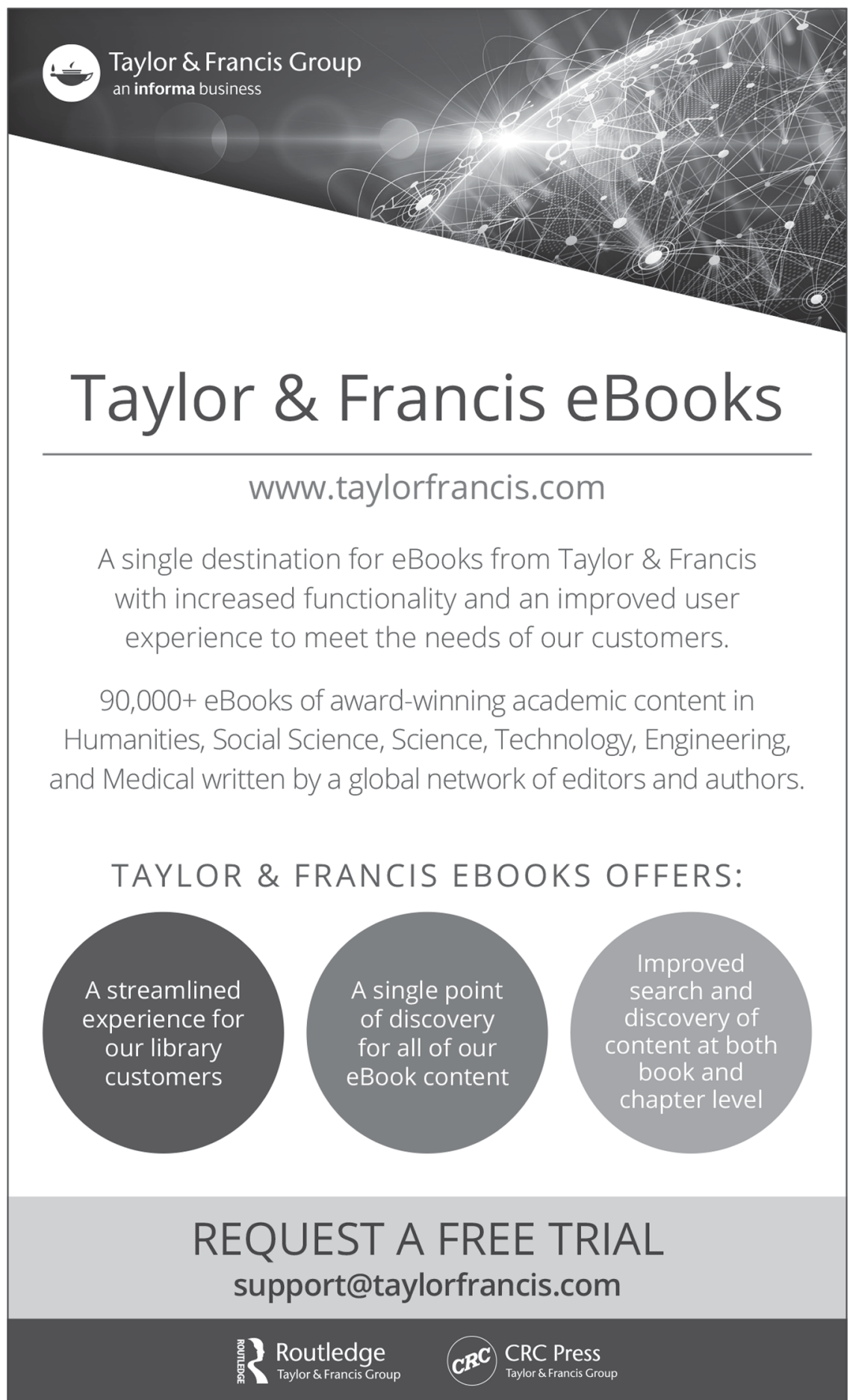

